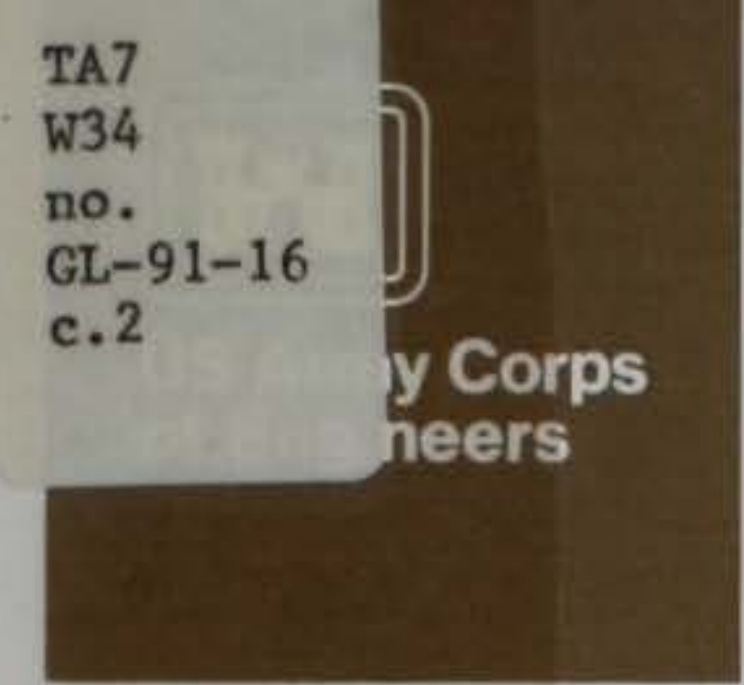

\title{
COMPACTION CONTROL OF EARTH-ROCK MIXTURES
}

\author{
by
}

Victor H. Torrey III, Robert T. Donaghe

Geotechnical Laboratory

DEPARTMENT OF THE ARMY

Waterways Experiment Station, Corps of Engineers

3909 Halls Ferry Road, Vicksburg, Mississippi 39180-6199

\section{US-CE-C PROPERTYOF THE UNITED STATES GOVERNMENT}

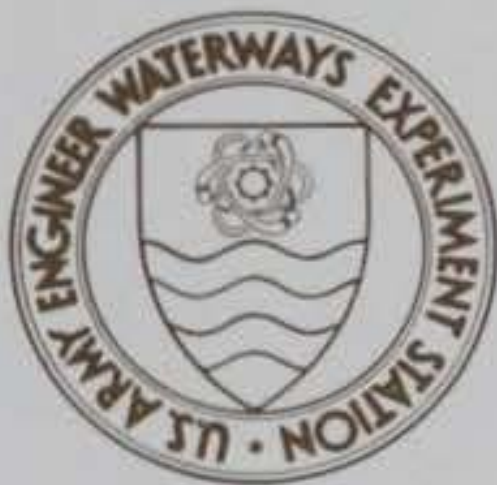

August 1991

Final Report

Approved For Public Release; Distribution Unlimited

RESEARCH LIBRARY

JS ARMY ENCINEER WATERWAYS

EYPEA:IINT STATION

VICKSBURG, MISSISSIPPI

Prepared for DEPARTMENT OF THE ARMY

US Army Corps of Engineers

Washington, DC 20314-1000

Under CWIS No. 32342 


\section{REPORT DOCUMENTATION PAGE}

Public reporting burden for this coliection of information is estimated to average 1 hour per response. including the time for reviewing instructions, searching exsting data sources. gathering and iliection Davis Highway, Suite 1204, Arlington, VA 22202.4302, and to the Otfice of Management and gudget, Paperwork fieduction Project (0704.0189). Waihington, DC 20503

\begin{tabular}{|l|l|l|}
\hline 1. AGENCY USE ONLY (Leave blank) & 2. REPORT DATE & 3. REPORT TYPE AND DATES COVERED
\end{tabular} 4. TITLE AND SUBTITLE

August 1991

Final report

Compaction Control of Earth-Rock Mixtures

CWIS No, 32342

6. AUTHOR(S)

V. H. Torrey III

R. T. Donaghe

7. PERFORMING ORGANIZATION NAME(S) AND ADDRESS(ES)

USAE Waterways Experiment Station

Geotechnical Laboratory

$3909 \mathrm{Halls}$ Ferry Road

Vicksburg, MS 39180-6199

9. SPONSORING/MONITORING AGENCY NAME(S) AND ADDRESS(ES)

US Army Corps of Engineers

Washington, DC 20314-1000

\section{SUPPLEMENTARY NOTES}

Available from National Technical Information Service,

5285 Port Royal Road, Springfield, VA 22161

\section{2a. DISTRIBUTION/AVAILABILITY STATEMENT}

Approved for public release;

distribution unlimited

13. ABSTRACT (Maximum 200 words)

This report addresses the general subject of compaction control of fills composed of gravelly soils, i.e., earth-rock mixtures, which exhibit welldefined moisture-density (compaction) curves. The effects on the compaction curve of the addition of gravel in increasing proportions are discussed. Compaction control methods currently employed by the US Army Corps of Engineers, US Bureau of Rreclamation, American Association of State Highway and Transportation officials, and the US Navy are discussed and compared. A new method of compaction control is described which is shown to be superior to those methods currently in use. The new method is based on either the minus $3 / 4$-in. or minus No.4 US Standard sieve size fraction and, in most cases, does not require large-scale compaction testing of the total material gradations.

\begin{tabular}{|c|c|c|c|}
\hline \multirow{2}{*}{$\begin{array}{l}\text { 14. SUBJECT TERMS } \\
\text { Compaction } \\
\text { Compaction control } \\
\text { Gravelly soils }\end{array}$} & \multirow{2}{*}{$\begin{array}{l}\text { Rock corrections } \\
\text { Soil testing }\end{array}$} & & \multirow{2}{*}{$\begin{array}{l}\text { 15. NUMBER OF PAGES } \\
212 \\
\text { 16. PRICE CODE }\end{array}$} \\
\hline & & & \\
\hline $\begin{array}{l}\text { 17. SECURITY CLASSIFICATION } \\
\text { OF REPORT } \\
\text { UNCILASSIEIED } \\
\end{array}$ & $\begin{array}{l}\text { 18. SECURITY CLASSIFICATION } \\
\text { OF THIS PAGE } \\
\text { UNCLASSIEIED } \\
\end{array}$ & $\begin{array}{l}\text { 19. SECURITY CLASSIFICATION } \\
\text { OF ABSTRACT }\end{array}$ & 20. LIMITATION OF ABSTRACT \\
\hline
\end{tabular}


This investigation is part of a study to improve understanding of the engineering properties and behavior of soils containing large particles and to develop laboratory testing procedures and fill compaction control methods which more accurately measure or predict those properties and behavior than methods currently in use. Funding for the work is provided by the Headquarters, US Army Corps of Engineers (USACE) under the Civil Works Research and Development (CWRD) Program work unit No. 32342, entitled "Testing LargeParticled Soils." The USACE Technical Monitor for this work unit is Mr. Richard F. Davidson, Directorate of Civil Works, Engineering Division, Geotechnical and Materials Branch, Soils Section, USACE, Washington, DC. The Program Manager is Mr. G. P. Hale, Chief, Soils Research Center (SRC), Soil and Rock Mechanics Division (S\&RMD), Geotechnical Laboratory (GL), US Army Engineer Waterways Experiment Station (WES), Vicksburg, MS. The designated Principal Investigator for CWRD work unit No. 32342 is Dr. Victor H. Torrey III, of the Soil Mechanics Branch (SMB), S\&RMD, GL, WES.

This report was prepared by Dr. Torrey and Mr. Robert T. Donaghe of the Soils Research Facility, SRC, S\&RMD, under the administrative supervision of Mr. William M. Myers, Chief, SMB. Dr. Don C. Banks is Chief, S\&RMD, and Dr. William F. Marcuson III, is Chief, GL. Technical editing and coordination of preparation of this report for publication were performed by Mrs. Joyce $H$. Walker of the WES Visual Production Center, Information Technology Laboratory.

COL Larry B. Fultori, EN, is the Commander and Director of WES.

Dr. Robert W. Whalin is the Technical Director. 
PREFACE

CONVERSION FACTORS, U. S. CUSTOMARY TO METRIC (SI)

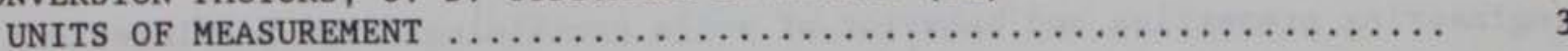

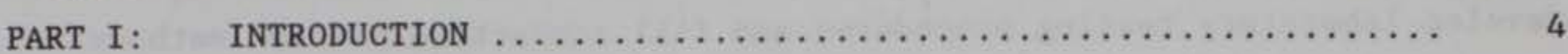

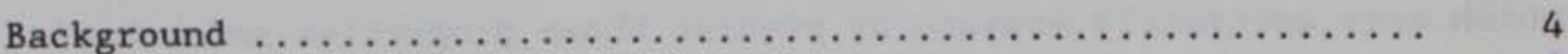

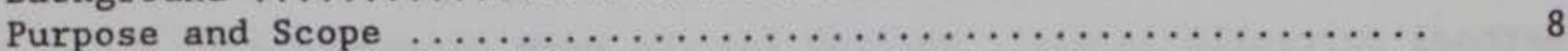

PART II: ACCURACY AND PRECISION OF THE COMPACTION TEST $\ldots \ldots \ldots \ldots \ldots .9$

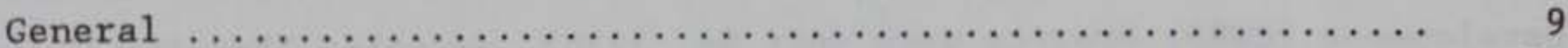

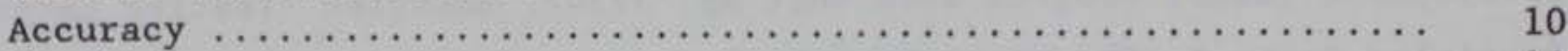

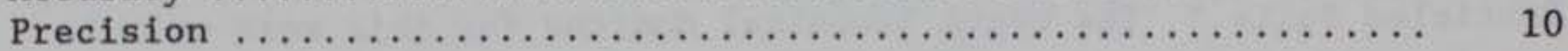

PART III: COMPACTION CONTROL METHODS $\ldots \ldots \ldots \ldots \ldots \ldots \ldots \ldots \ldots \ldots \ldots \ldots \ldots \ldots \ldots \ldots \ldots$

Specifications . . . . . . . . . . 18

General Comments on Compaction Control ................... 19

Methods for Estimating Maximum Dry Density and Optimum Water

Content for the Fill Sample $\ldots \ldots \ldots \ldots \ldots \ldots \ldots \ldots \ldots \ldots \ldots \ldots, 21$

PART IV: GENERAL EFFECTS OF GRAVEL ON THE MOISTURE-DENSITY CURVE .... 29

PART V: $\quad$ ACCOMMODATING OVERSIZED PARTICLES $\ldots \ldots \ldots \ldots \ldots \ldots \ldots \ldots \ldots$

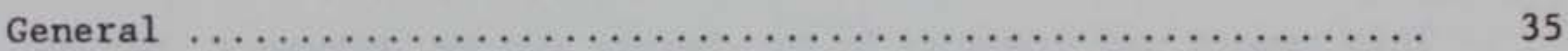

Testing of Altered Gradations Perceived as Simulations of the

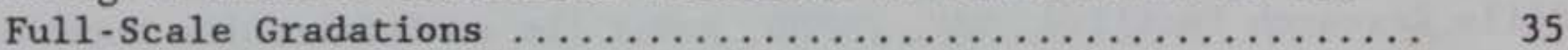

Correcting Maximum Dry Density and Optimum Water Content to

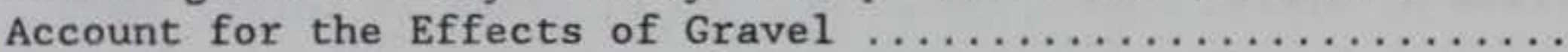

Basing the Specifications on the Compacted State of a

Fraction of the Total Material $\ldots \ldots \ldots \ldots \ldots \ldots \ldots \ldots \ldots \ldots \ldots \ldots$

PART VI: A UNIFIED VIEW OF THE EFFECTS OF GRAVEL ON THE COMPACTION OF THE MINUS NO. 4 OR MINUS $3 / 4$-IN. FRACTION $\ldots \ldots \ldots \ldots \ldots$

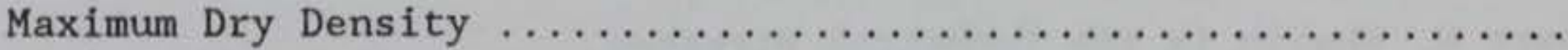

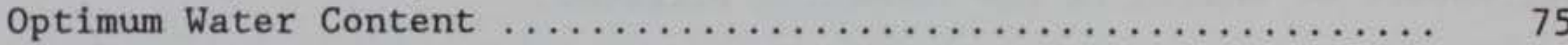

Applying the Density Interference Coefficient and Optimum

Water Content Factor in Compaction Control .............. 79

PART VII: CONCLUSIONS AND RECOMMENDATIONS $\ldots \ldots \ldots \ldots \ldots \ldots \ldots \ldots \ldots \ldots, 81$

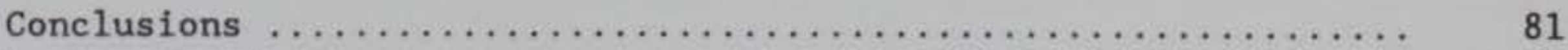

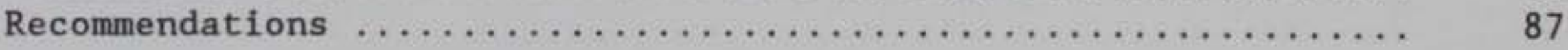

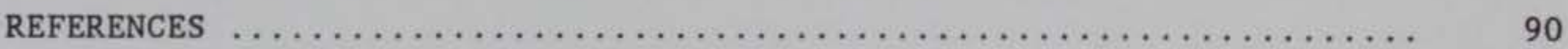

TABLES $1-15$

FIGURES $1-92$

APPENDIX A: LISTING OF EARTH DAM CRITERIA REPORTS $\ldots \ldots \ldots \ldots \ldots \ldots \ldots$ A1

APPENDIX B: DETERMINING THE WATER CONTENT OF THE OVERSIZED

FRACTION . . . . . . . . B 


\section{CONVERSION FACTORS, NON-SI TO SI (METRIC) \\ UNITS OF MEASUREMENT}

Non-SI units of measurement used in this report can be converted to SI (metric) units as follows:

Multiply
cubic feet
cubic inches
feet
foot-pounds (force)
inches
pounds (force)
pounds (mass)
pounds (mass) per cubic foot
square inches

$\frac{\text { By }}{0.02831685}$
16.38706
0.3048
1.355818
2.54
4.44822
0.4535924
16.01846
6.4516

To Obtain

cubic metres

cubic centimetres

metres

metre-newton or joules

centimetres

newtons

kilograms

kilograms per cubic metre

square centimetres 


\section{COMPACTION CONTROL OF EARTH-ROCK MIXTURES}

\section{PART I: INTRODUCTION}

\section{Background}

1. Before even beginning to address the subject of this report, it is appropriate to place the reference to earth-rock mixtures in perspective as is necessary to establish a distinction between such materials and those termed as "rockfill." Fortunately, at least within the experience of the US Army Corps of Engineers (USACE), this is a practical undertaking because review of earth and/or rockfill dam projects permits a general, although not precise, distinction. The authors recognize the variations in those project records which in some cases may contradict some aspects of the following definitions. Nonetheless, for the purposes of this report, earth-rock mixtures are coarsegrained* (less than 50 percent passing the US Standard No. 200 sieve), grave1 or gravelly, cohesive and cohesionless materials which are "designed" as compacted fill by assessment of their properties through laboratory testing to establish fill compaction specifications for placement water content and density if sufficient fines are present or for relative density if the soil is cohesionless. Furthermore, the compaction of these materials is controlled in the fill operation by regular comparison of field measurements of those parameters to the specified values or ranges in values. In contrast, rockfill is typically dealt with in a more qualitative than quantitative manner with placement and compaction procedures determined by means of test fills and quarries (Hammer and Torrey 1973) to identify the most efficient quarrying, processing and handling operations, to select the most efficient combination of equipment versus loose lift thickness, and to obtain (usually) a "freedraining" compacted mass. During construction, the selected procedures for rockfill are ordinarily followed without regular attention to fill testing unless apparently adverse changes are noted in materials or compacted fill qualities. Rockfill is typically cohesionless and composed of larger

* Terms used in this report relative to classification of soils or fractional components are according to the Unified Soil Classification System. 
particles (say, up to 24 in. ${ }^{*}$ ) and compacted in much thicker lifts (say, upto 36 in.) than earth-rock mixtures which often contain plastic fines and are seen in the case histories to have been restricted to a maximum particle size of about $6 \mathrm{in.}$ (either naturally or by removal of particles in excess of that size) and compacted in lift thicknesses of less than $12 \mathrm{in.}$

2. Laboratory tests to obtain moisture-density relationships for soils containing large particles, i.e., earth-rock mixtures, have been both problematical and questionable over the years. The problem in dealing with such materials arises from the fact that if the full-scale gradation is to be tested, the size of the laboratory test specimen must be sufficiently large to assure assessment of the properties and/or behavior of the mixture. There seems to be general, although informal, agreement within the profession in this country that the ratio of test specimen diameter to largest particle size should be no lower than 5 or 6 to achieve a good test on the mixture. Both USACE (1970) and American Society for Testing and Materials (1991a and 1991b) methods conform to this concept. Working with a ratio of 5 or 6 leads to what would be conventionally considered large test specimens (in excess of 6 in. in diameter) as the largest particle size begins to exceed 1 in. Testing of larger specimens entails the need for larger and more expensive laboratory hardware, higher capacity compaction and/or loading mechanisms, special processing and handling equipment, more spacious facilities, specialized instrumentation, and lots of hard manual labor. Therefore, beginning years ago, as one laboratory after another began to encounter these realities in testing soils containing large particles, methods were developed or adopted on faith which were believed to provide adequate estimates of full-scale gradation properties but which also circumvented testing of large specimens of the fullscale materials. Simplistically, the avoidance procedures have included practices such as discarding the larger particles (scalping), or scalping and then replacing the "oversized" fraction with an equal portion by weight of manageable sizes, or even the creation of a "parallel" gradation with a smaller maximum particle size. Formal research to assess the reliability of methodologies for testing earth-rock mixtures has been very sporadic and has mostly fallen to organizations engaged in regular major design and construction activities involving these materials such as the USACE, US Bureau of

* A table of factors for converting non-SI units of measurement to SI (metric) units is given on page 3 . 
Reclamation (USBR), and some state agencies (including universities). However, because of the expense, time-consuming nature of the work, and the many variables commensurate with earth-rock mixture research, sporadic efforts have not sufficed to eliminate many of the basic questions. This fact is typified by the current realization in the profession that the long-standing and popular procedure of scalping with replacement to reduce maximum particle size and, thus, test specimen diameter, should not generally be assumed to yield test results satisfactorily indicative of full-scale gradation properties or behavior. This is not to say that there are no materials encountered for which scalping and replacing may be adequate, but that this procedure should not be presumed as ordinarily adequate.

3. At present, the USACE practice (USACE 1970, Appendix VIA) in performing laboratory compaction tests on earth-rock mixtures containing sufficient fines to produce a well-defined moisture-density curve entails a 12-in. diam mold and an 11.5-1b hand-held rammer. The maximum mold size of 12 in. confines the test to a maximum particle size of 2 in. It is stated that plus 2-in. fractions constituting less than 10 percent by weight of the total material may be scalped, i.e., removed and discarded. If more than 10 percent by weight of the total material is of particle sizes larger than 2 in., the plus 2-in. sizes are scalped and replaced with an equal weight of material between the 2 -in. and No. 4 sieve sizes. The gradation of the replacement material must be the same relative gradation as that of the total sample between the 2 -in. and No. 4 sieve sizes. In the case of cohesionless materials for which the concept of relative density is applicable (less than 5 percent minus No. 200 sieve sizes), USACE (1970), Appendix XII, Vibrated Density Method specifies either a 6 -in. or 11 -in. mold diameter $(0.1 \mathrm{cu} f t$ and $0.5 \mathrm{cu} \mathrm{ft}$, respectively). The 6 -in.-diam mold is to be used if the maximum particle size is less than $1-1 / 2$ in. and the $11-i n$. mold is to be used if the maximum particle size is less than $3 \mathrm{in}$. If the material contains less than 10 percent by weight of plus 3 -in. sizes, they are scalped. If the material contains more than 10 percent by weight of particles larger than $3 \mathrm{in}$, the test is relegated to a research stature and no procedure is suggested.

4. In summary, some of the problems associated with the current USACE procedures (USACE 1970) as described above are as follows:

a. The compaction test for earth-rock materials exhibiting moisture-density curves is restricted to a maximum particle sizes of 2 in. Many commonly encountered earth-rock mixtures 
have plus 2 -in. fractions which exceed 10 percent of the total material by weight.

b. The compaction test for earth-rock mixtures requires the use of an 11.5-1b hand-held rammer in the 12-in. mold. This procedure has drawn considerable criticism from USACE Division Laboratories, USACE field construction quality assurance laboratories, and contractor quality control laboratories because they consider it too time-consuming and labor intensive.

c. The scalping with replacement procedure for earth-rock mixtures containing more than 10 percent by weight plus 2 -in. sizes is now considered undependable as a general "modelling" method to obtain compaction parameters of full-scale gradations.

d. There appear to be differences in moisture-density curves obtained in large molds resulting from the larger mold diameter itself, different hammer weights and their relative foot areas, differences in layer thicknesses, or other equipment/procedural factors.

e. The relative density test procedure allows up to 3 -in. particles in an 11-in.-diam mold. There has been no definitive research to ascertain the effects of this practice.

5. Looking back to the generalized definition of earth-rock mixtures provided in the first paragraph, the typical gradation ranges seen in project files for such materials lead to a fortuitous possibility to arrive at a practical answer concerning the maximum particle size which laboratory tests should accommodate. In overview, the authors observe that it has been relatively infrequent for earth-rock mixtures used in controlled USACE fills to exhibit more than 10 percent by weight of sizes in excess of $3 \mathrm{in.}$. If it is accepted that up to 10 percent by weight of a material containing a significant gravel fraction can be scalped on the 3-in. sieve (plus 3-in. sizes discarded) without introducing significant error in compaction parameters, then laboratory compaction test procedures validated in molds up to $18 \mathrm{in}$. in diameter would appear to ordinarily suffice. Such a laboratory compaction test procedure was developed by Torrey and Donaghe (1991) as a part of the Civil Works Investigational Study (CWIS) 32342 objectives.

6. In consideration of the scale of the problems in the laboratory environment, it is no surprise to discover that earth-rock mixtures also present a plethora of "challenges" in the field construction environment. Of course, the field laboratory faces the testing uncertainties previously mentioned. Next comes the requirement for a sufficiently precise, efficient method for determining the as-compacted fill density and fill water content of soils containing large particles. Then, there is the need to compare those 
values of fill density and water content to appropriate values of maximum dry density and optimum water content to assure that specifications are met, i.e., a quality control or assurance procedure. Because of the rate of fill placement in the construction of large fills, it is not feasible to expect to develop complete moisture-density curves for samples of earth-rock mixtures from each fill density test location. So, it is imperative that the compaction control methodology not only be shortcut in nature but also sufficiently precise to confirm the specified attributes of the fill.

7. The major laboratory investigation into the compaction characteristics of earth-rock mixtures mentioned above as a previous task under CWRD 32342 (Torrey and Donaghe 1991) was directed at achieving the following objectives:

a. The development of standard effort compaction test procedures utilizing 6-, 12- and 18-in.-diam molds and mechanical compaction equipment for earth-rock mixtures having maximum particle sizes up to $3 \mathrm{in.}$ and containing sufficient fines (minus No. 200 sieve sizes) to exhibit well-defined moisture-density curves.

b. The determination of the extent to which Equations B-1 and B-2 of USACE (1977), Appendix B, may be utilized to predict the maximum dry densities and optimum water contents, respectively, of full-scale gradations from tests performed on finer fractions of the full-scale materials in smaller diameter molds.

The report of results of that investigation was so voluminous that it was decided that the subject of fill compaction control of earth-rock mixtures would be addressed in this separate document. Many of the pertinent findings of the previous work will be referred to or reiterated herein. For the sake of brevity, those findings will be used without explanation of any procedures or techniques involved in the testing program which produced them. The reader is referred to Torrey and Donaghe (1991) for such details.

\section{Purpose and Scope}

8. This report includes assessment of the methods currently in use by the USACE and other agencies to control the compaction of fills composed of earth-rock mixtures, provides information to the field elements of the USACE concerning good practice, and recommends new methods for controlling water content and density which are deemed worthy of field trial. 


\section{General}

9. Before proceeding to discuss the various approaches to compaction control of earth-rock mixtures, attention is directed to some harsh realities about those fundamental reference values so casually referred to as "THE" optimum water content and "THE" maximum dry density of a soil for a given compactive effort. Both of these parameters are the result of the subjective judgment of an individual in the fitting of a compaction curve to data typically exhibiting some scatter. Furthermore, the usual test consists of five data points at different water contents which are accepted as sufficient if a smooth curve appears to reasonably fit. Any scatter which might result if replicate specimens were compacted at each given water content is not indicated unless a point appears to be "out of line" with the other four and a "check" point is ordered. If an experienced and careful technician performs a number of five-point compaction tests on the same material fitting each data set independently with a compaction curve, it is to be expected that ranges in values of optimum water content and maximum dry unit weight will result. Suppose a second technician in the same laboratory is also required to perform multiple tests on the same material using the identical equipment and procedures as the first technician. If the results obtained by both technicians are combined, the total ranges in values of optimum water content and in values of maximum dry unit weight would be expected to be larger than those obtained by either individual. If the two technicians are employed in different laboratories, the observed ranges in the combined data would be expected to be still greater.

10. The occurrence of differences in results obtained by replicate application of a "standard" method to the same material can be addressed within the statistical concepts of accuracy and precision. The applicability of these two concepts to results of compaction tests will be discussed below after their definitions and usages prescribed by the American Society for Testing and Materials, Designation E 177-86 (ASTM 1991c). 


\section{Accuracy}

11. According to ASTM (1991c), accuracy is defined according to two schools of thought. One definition is the closeness of agreement between an accepted reference value and an individual test result. The second definition is the closeness of agreement between the accepted reference value and the average of a large set of test results obtained by repeated applications of the test method, preferably in many laboratories. Where the compaction test is concerned, it makes no difference which definition is accepted because there exist no accepted reference values of maximum dry density or optimum water content for any given soil. In other words, there is no way to identify which values are the "correct" values. Therefore, the concept of accuracy is not applicable to the compaction test.

\section{Precision}

12. Precision of a measurement process refers to the degree of mutual agreement between individual measurements from the process. This concept does apply to the compaction test. Furthermore, precision of the compaction test can be categorized after the fashion of the cases mentioned in paragraph 9 above as follows:
a. Single-operator precision.
b. Multioperator precision.
c. Multilaboratory precision.

These precision cases are defined as follows:

a. Single-operator precision: A measure of the greatest difference between two test results that would be considered acceptable when properly conducted determinations are made by one operator on portions of a material that are intended to be identical, or as nearly identical as possible.

b. Multioperator precision: A measure of the greatest difference between two test results that would be considered acceptable when properly conducted determinations are made by more than one operator in the same laboratory on portions of a material that are intended to be identical, or as nearly identical as possible.

c. Multilaboratory precision: A measure of the greatest difference between two test results that would be considered acceptable when properly conducted determinations are made by two different operators in two laboratories on portions of a material 
that are intended to be identical, or as nearly identical as possible.

In this vein, the ASTM currently cites (see Table 1) single-operator and multilaboratory precision standards in Designations D 698-78 (ASTM 1991a) and D 1557-78 (ASTM 1991b) for results of standard effort and modified effort compaction tests, respectively, employing 4- and 6-in. diam molds. There are no current ASTM standards for large-scale compaction tests for earth-rock mixtures. ASTM currently bases precision limits on the statistical parameter "difference two-sigma limit" (see ASTM 1991c) which is calculated as follows:

Difference $2 \sigma$ imit $=1.96 \sqrt{2} \sigma=2.77 \sigma$

where $\sigma$ is the standard deviation

Given that a variable is normally distributed (random), the probability that any two numbers drawn from the population will not differ by more than some amount can be calculated. Also, for a normally distributed variable, about 95 percent of the values will fall within the range of \pm 20 of the mean value. The intended practical significance of the difference two-sigma limit is that statistically there is about a 95 percent probability (the reason that $2 \sigma$ is used in the term) that any two numbers drawn at random from among all the values will not differ by more than 2.770 . The ASTM standard then takes the difference two-sigma limit of 2.770 and expresses it as a percentage of the mean value of the variable. The impact of ASTM precision standards for 4-in. and 6-in. mold diameters should they be applied to larger diameter mold tests on a typical earth-rock material can be indicated. A typical earth-rock mixture may exhibit a maximum dry density around $130 \mathrm{pcf}$ and an optimum water content around 7 percent. The single-operator precision stated in Table 1 for maximum dry density would be 1.9 percent of 130 pcf or almost 2.5 pcf absolute difference between the two values. The single-operator precision of Table 1 for optimum water content would be 9.5 percent of 7.0 percent or 0.7 percentage points absolute difference between the two values. Considering the multilaboratory case, such as between the USACE quality assurance lab and the contractor's quality control lab, 4.2-pcf absolute difference in maximum dry density weight and 1.0 percent absolute difference in optimum water content 
would be acceptable under ASTM current standards. The key phrase in the definition(s) of precision is "when properly conducted determinations are made.... on portions of a material intended to be identical." A proper testing program to determine precision limits for the compaction test is a very costly and complex undertaking. There must be careful attention to test materials, all associated methods such as moisture curing of specimens, calibration of all equipment to the same reference standards, etc. After all, the question is the repeatability of results from the test method, not the variability in laboratory practice. It is logical that multilaboratory precision cannot be addressed until the question of single-operator precision has first been resolved. It makes no sense to accept any values in the multi-laboratory study that have not met the single-operator precision. This would dictate acceptable replicate single-operator tests in each participating laboratory with perhaps the average values reported for the multi-laboratory case. To the best of the authors' knowledge the current ASTM precision standards were not derived in this manner. The ASTM is currently considering the need to rigorously address precision of laboratory soils tests.

13. It is valuable at this point to interject a review of three testing programs pertinent to the question of precision in compaction testing. However, none of these studies meet all the criteria stated above as necessary to establish general multi-laboratory precision standards for the compaction test.

14. The first study was initiated in 1964 under the auspices of the American Council of Independent Laboratories (ACIL) and was aimed at obtaining an indication of variation in test results among commercial laboratories pertaining to Atterberg limits, optimum water content and maximum dry density by Standard and Modified efforts, specific gravity of solids, and grain-size distribution. With respect to compaction tests, the only requirement imposed was the use of ASTM Designation D 698-58T, Method A, for standard effort and Designation D-1557-58T, Method A, for modified effort. There were no other controls imposed. To achieve these objectives, three "standard" soils were selected to be provided to all participating commercial laboratories. The three soils were designated as Vicksburg loess (ML), Vicksburg lean clay (CL) and Vicksburg buckshot clay $(\mathrm{CH})$. Preparation of the standard samples was accomplished by WES at the request of and assisted by ACIL. Under the supervision of ACIL personnel, the three materials were carefully processed at 
WES and placed in 333 sealed containers weighing $80 \mathrm{lb}$ each and stored under cover to await shipment to the requesting commercial laboratories. Three "umpire" laboratories were designated by ACIL and included WES, US Bureau of Public Roads, and Massachusetts Institute of Technology. These umpire laboratories ran 4 to 5 replicate compaction tests on each sample. Approximately 100 commercial laboratories participated in the program although all laboratories did not perform all of the test suite. However, 98 of the labs performed standard and modified effort compaction tests on the ML and CH samples and 97 labs developed compaction curves for the CL sample. The discrete data obtained from the program are reported and analyzed statistically by Hammitt (1966). Figures 1 through 3 present the results of the standard effort tests obtained by the commercial laboratories for the ML, CL, and CH samples, respectively, as replotted by the authors. Also shown in these figures and in Table 2 are the ranges and mean values obtained by the umpire labs. The modified effort data are not treated in detail because they were not appreciably different in scatter patterns. The statistical summaries for the commercial laboratory results are given in Tables 3 through 5 . The scatter of the data seen in Figures 1 through 3 reveals the magnitude of the problem of specifying acceptable precision for compaction test parameters based on an essentially uncontrolled testing program. It is obvious from Figures 1 through 3 that some laboratories did not properly conduct the test. But, how many of the test data are the result of poor practice? If the acceptable precision is based on the standard deviation for all the test data for a given soil type among Figures 1 through 3, it will be a "sloppy" standard. Table 6 shows the difference two-sigma precision limits for maximum dry density and optimum water content calculated for each of the ACIL data sets of Figures 1 through 3 as dashed boxes. The precision limits specified by ASTM Designation D 698-78 are also shown in these figures. Scatter of the compaction data clearly varies with soil plasticity with the $\mathrm{CH}$ soil exhibiting the greatest dispersion (largest standard deviations) and the ML soil exhibiting the least. Note from Table 6 that while the difference two-sigma limit for maximum dry density in pcf obviously must track the trend in standard deviation, the limit stated in terms of percent of mean value does not because of the relative values of the mean maximum dry densities. Also note that use of a single precision range as a percent of mean value as the ASTM currently specifies, runs counter to the trends for maximum dry density indicated by the ACIL study. In other words, a 
fixed precision for all soil types would force a smaller acceptable difference between two values of maximum dry density obtained for a $\mathrm{CH}$ soil which is most difficult to obtain consistent values for and a more generous difference allowance for ML and CL soils which showed less data dispersion. If the precision limits indicated for the $\mathrm{CH}$ soil were adopted for all soil types, this would aggravate the already sloppy practice of accepting all data as equally correct in calculating precision limits. It is seen from Figures 1 through 3 that the current ASTM multilaboratory precision limits for maximum dry density are somewhat more restrictive compared with the values calculated from the ACIL data, especially for the $\mathrm{CH}$ soil. With respect to multilaboratory precision of optimum water content, it is seen that the current ASTM standard is similarly more restrictive than the limits calculated using the ACIL data. However, the optimum water content precision limits stated as a percent of mean value are the greatest for the more problematical $\mathrm{CH}$ soil because that soil exhibits the highest values of optimum water content. It is to be noted that the standard deviations for both maximum dry density and optimum water content from the ACIL data generally exceed the specified maximum values of ASTM Designation D 698-78 (see Table 1). An inconsistency exists in the ASTM standard in that both standard deviation and difference two-sigma precision limits as a percent of mean value are stated for the multi-laboratory case. If the standard deviation restriction is accepted as the reference, then the precision range as a percent of mean value must be a variable because the mean value varies (or vice versa). If the precision was stated as a range in maximum dry density in pcf or as a range in optimum water content in percentage points, there would be no inconsistency since these are fixed values calculated as $2.77 \sigma$.

15. Concurrently with the ACIL study among commercial laboratories, the USACE decided to have its Division Laboratories also test the standard soil samples. Strohm (1966) reports the results obtained among ten Division Laboratories. Figures 4 through 6 show the standard effort compaction data for the standard soils. Since this study predated the first edition of EM 1110-2-1906 which standardized equipment, the data reflect a mix of compaction equipment as indicated in the figures. Scatter in the USACE data for both optimum water content and maximum dry density increased with plasticity of fines as did the commercial lab results previously discussed. As was done for the commercial lab results, both the difference two-sigma precision limits 
calculated from the standard deviations of the data and those specified currently by ASTM are shown in Figures 4 through 6 as dashed boxes. The values calculated from the standard deviations of the data are tabulated in Table 7. For the standard ML soil, precision calculated as above would be 9.8 percent of the mean value for optimum water content and 2.1 percent of the mean value for maximum dry density. For the standard CL soil, the precision was 13.2 percent of the mean value for optimum water content and 2.7 percent of the mean value for maximum dry density. For the standard $\mathrm{CH}$ soil, precision was 22.3 percent of the mean value for optimum water content and 4.0 percent of mean value for the maximum dry density. So, on the average, the multilaboratory precision achieved by the USACE labs for optimum water content was about equivalent to the current ASTM standard but the precision achieved for maximum dry density was equal to or better than the current ASTM requirements (despite variation in equipment).

16. It is reasonable to consider the ACIL umpire laboratory results as a multilaboratory study in its own right. Unfortunately, as seen in Table 3 , standard deviations were not reported for those data. However, the ranges of the data were reported. For data which are normally distributed (random variable), 99.7 percent of the data falls within \pm 3 standard deviations $(\sigma)$ of the mean and 95.5 percent of the data falls within $\pm 2 \sigma$. Taking a conservative approach, a very crude estimate of the standard deviations of the umpire laboratory data can be made by taking the respective ranges to be equivalent to 4 times the respective values of $\sigma$. If this is done and difference twosigma precision limits are calculated for the ACIL standard soils accordingly, the limits seen in Table 8 result. From Table 8 it is seen that the multilaboratory precision limit stated as a percent of mean value for maximum dry density are only about one-half the current ASTM standard while the limits calculated for optimum water content are anywhere from about one-fourth to one-half the current ASTM value.

17. The third study (Sherwood 1970) consisted of single-operator, multioperator and multilaboratory compaction and soil classification testing organized by the British Road Research Laboratory (RRL) involving itself and 39 other government, university, and private testing laboratories. The only condition imposed upon the laboratories was that British Standard 1377:1967 was to be employed for all test methods. The soils selected by RRL for the study were a sandy clay, $\mathrm{CL},(\mathrm{LL}=36, \mathrm{PI}=19)$, Gault clay, $\mathrm{CH},(\mathrm{LL}=75, \mathrm{PI}=26)$, 
and Weald clay, $\mathrm{CH},(\mathrm{LL}=68, \mathrm{PI}=25)$. These materials were carefully processed and batched for distribution to the participants in a fashion similar to that used for the "standard" soils of the ACIL study. Compaction tests equivalent to standard and modified efforts were performed among the participants. Thirty seven of the 40 labs provided results for the sandy clay (CL) and 38 labs tested the Gault and Weald clays $(\mathrm{CH})$. The results of the standard effort tests are shown in Figures 7 through 9. The difference two-sigma precision limits calculated from the standard deviations of the data for the various cases addressed by RRL are given in Table 9 . The calculated precision limits and the ASTM precision limits relative to the multilaboratory data are shown as dashed boxes in Figures 7 through 9. Figures 7 through 9 show that the RRL data exhibit scatter similar to that seen in the ACIL study for the CL and $\mathrm{CH}$ soils. The standard deviations relative to maximum dry density for the RRL data were slightly lower than those seen for the ACIL data. These comparative dispersions were not strictly consistent with differences in plasticity index since the RRL clay (CL) was more plastic than that tested in the ACIL study, but the two clays $(\mathrm{CH})$ of the RRL study were both less plastic than that tested in the ACIL study. The dispersion of the optimum water content data was about the same for the two CL soils between the two studies but the standard deviations for the two RRL clay $(\mathrm{CH})$ soils were greater than that for the ACIL clay $(\mathrm{CH})$ soil.

18. The single- and multioperator precisions obtained by the RRL are also shown in Table 9. An expectable trend in improving precision is seen from multi-laboratory to multioperator to single-operator for both maximum dry density and optimum water content for the Gault clay which was the only soil replicate tested by the single-operator. It is seen by comparing Tables 8 and 9 that it appears that the three ACIL umpire laboratories probably at least matched the RRL single-operator precision for both compaction parameters.

19. In speaking of relative dispersions of the data among the cases discussed above, there is more to the question than simple comparisons of the numbers. Figure 10 reveals an apparent relationship between standard deviations and numbers of laboratories participating for the $\mathrm{CL}$ and $\mathrm{CH}$ soils. The RRL data seem to fit well with the ACIL data probably because the CL and $\mathrm{CH}$ soils tested by RRL were not to different from the ACIL soils with respect to classification indices. The authors suspect that the lower standard 
deviations achieved by the 10 USACE Division Laboratories and the estimated values for the ACIL umpire labs actually reflect a greater consistency of practice and care exercised in performance of the tests by those labs as compared with the "catchall" nature of the RRL and ACIL commercial lab results. Anyway, it appears that two laboratories performing the test carefully with properly calibrated equipment can achieve results much closer together than indicated by precision limits derived from data produced by a large number of organizations. The authors suggest that it is reasonable to expect that two well coordinated and calibrated laboratories should fall within 2.0 pcf of one another in maximum dry density and within 1.0 percentage point in optimum water content. Based on the experience with large-scale compaction tests for earth-rock mixtures, the authors are of the opinion that these precision ranges are also practical for soils containing gravel.

20. Despite the drudgery of the foregoing discussion, the authors felt it to be important for the purposes of this report to precede any treatment of compaction control methods with some facts about the consistency of results obtained from a compaction test procedure, particularly between two laboratories. It was intended to bring the reader to a realization of the importance of preconstruction and during construction coordination and calibration both within and between the USACE quality assurance and the contractor quality control laboratories. 


\section{Specifications}

21. There are two approaches to writing specifications for the compaction of earthen fills consisting of clayey and silty soils which exhibit an optimum water content associated with a maximum dry density as obtained by an impact compaction test. The first is termed a "method" specification and the other is termed an "end result" specification. The method specification includes an acceptable range in placement water content usually referenced in percentage points with respect to optimum water content and also dictates the compactive effort to be applied. The compactive effort is specified through a statement of placement loose lift thickness, number of passes of a specific piece of equipment (which is also a specification of weight, directly or indirectly) and the maximum speed of that equipment. Therefore, the dictation of compactive effort amounts to requiring a certain method of compaction and hence the specification type gets its name. The end result specification is so named because it only states the required results in terms of percent compaction or a minimum compacted dry density. Placement water content may or may not be included in an end result specification. These two approaches spring from the relative degree to which water content and density and the compaction method affect the properties of the compacted material required to satisfy performance and safety criteria for the fill. Within the USACE, these two specification types have generally been identified in the minds of experienced personnel according to whether the work is a civil works or military project. The method specification has been most associated with civil works because it has been within that domain that most large earth and rock-fill dams have been constructed. These large embankments along with other water retention or water control projects not only require careful attention to the achievement of a variety of properties of the compacted material which reflect water content, density, and the compaction method but also typically demand special attention to the compaction method employed in such critical locations as foundation, abutments and areas immediately adjacent to buried structures. The end result specification has been identified with military projects which have typically consisted of military base facilities including airfields. These projects have consisted of items such as utility trench backfill, 
roadway, and airfield pavement subbases/subgrades, low-rise structure foundations, etc. It is not unusual that compacted density alone yields satisfactory material properties for these constructions so that intense attention to specific combinations of water content, density and compaction method are not required. Therefore, the simpler end result type of specification has been more often than not the most suitable for such project items. So, the distinction between the two types of specifications is purely coincidental with the usual and different characteristics and technical requirements of civil works as opposed to military projects even though there are those who argue the relative merits of the two concepts as if they must compete. The truth is that there is a place for both types of specifications as has been clearly identified in the broad experience of the USACE depending on the specific performance requirements of the compacted material. Indeed, there have been both civil works and military projects which have utilized both types of specifications for different project features. Where compaction control of earth-rock mixtures is concerned, it may make no difference which type of specification is employed since, for both cases, there may remain the needs to cope with the special problem of oversized particles in determining the state of compaction. The exception to this statement arises in an end result specification case if the soils can be easily categorized and a minimum compacted dry density can be specified for each category. In such cases, the fill density test provides a direct assessment of the adequacy of compaction. The remainder of this report will presume that both water content and density of the fill must be controlied without reference to any particular type of specification.

\section{General Comments on Compaction Control}

22. Fill density tests using direct or indirect methods and direct or indirect water content determinations on the total sample or a finer fraction (assuming the absorption of the gravel fraction as its water content) have ordinarily been used to obtain the as-compacted parameters (see USACE 1977 , paragraph 5-10). The specifications on range in placement water content and the desired percent compaction have been based on total material, some finer fraction (usually minus No. 4 or minus $3 / 4-i n$. fraction) of the total material or on scalped/replaced (usually on the $3 / 4-i n$. sieve) gradations. Most often, 
the specifications and the means of relating the as-compacted values values to the specifications have avoided dealing with the full-scale materials. For example, the specified range for water content and the value of minimum desired percent compaction may be based on the optimum water content and maximum dry density for a fraction of the total material. In general, the selected fraction has been either the minus No. 4 fraction or the minus $3 / 4$-in. fraction. The maximum dry density and optimum water content of the fraction may then be estimated using a one- or two-point compaction test (USACE 1977, Appendix B) on the fraction of the total material taken from the location of the fill density test. Then, the dry density and water content of the total fill sample are corrected according to USACE (1977), Appendix B, Equations B-1 and B-2 for the percent of total material by weight larger than the No. 4 or 3/4-in. sieve (termed "oversize") to obtain the dry density and water content of the fraction, respectively. Finally, the corrected fill values are compared to the optimum water content and maximum dry density of the fraction to check conformance to the specifications. Another example would be the use of a scalping with replacement procedure which is currently permitted in USACE (1970) to reduce the maximum particle size of the total materials for development of the compaction specifications during design. Subsequently, in the fill control procedure, use of the one- or two-point compaction test on scalped and replaced specimens of the total material taken from the location of the fill density test is assumed to be directly equivalent to testing of the total gradation. That is, the fill water content and density are compared directly with the scalped/replaced optimum water content and maximum dry density. So, the use of a fraction of the total material is based on a correction procedure while the use of scalping/replacement is predicated on a modelling of the total material. It has been shown by recent research that the scalping with replacement procedure does not satisfactorily model the total material. This will be discussed in more detail subsequently herein. The USBR Rapid Compaction Control Method (USBR 1989a) which has been occasionally employed by the USACE is a shortcut method for obtaining the deviation of the minus No. 4 fraction fill water content from its optimum value and the percent compaction of that fraction. Since the USBR rapid method is performed on the minus No. 4 fraction, corrections must also be applied if gravel is present in the total material. All of the above practices assume that the engineering properties and behavior of the total material will equal or exceed those (in 
terms of design requirements) of the selected fraction or scalped-replaced gradation when that fraction or scalped-replaced gradation meets the specified values. In cases where the specifications and desired percent compaction have referred to the total material, it has usually been the practice to correct the optimum water content and maximum dry density of the fraction estimated by the one- or two-point method to estimate the values for the total material. In this case, the corrections of USACE (1977), Appendix B, are applied in the opposite direction as compared to the case where the specifications are based on a fraction.

\section{Methods for Estimating Maximum Dry Unit Weight and Optimum Water Content for the Fill Sample}

\section{General}

23. It makes no difference which methods for estimating maximum dry density and optimum water content of the total fill sample or a fraction thereof are used as long as they are proved to be of acceptable precision. That is an obvious statement, but probably represents the most frequent serious shortfall in past compaction control practices. Years ago, compaction control data from over 100 USACE dams were collected in the files of WES in connection with preparation of so-called Earth Dam Criteria Reports under the auspices of the Headquarters USACE. A total of 67 of these reports summarizing pertinent site, design and construction data for each dam were issued by WES between 1960 and 1974. A list of those reports is given in Appendix B. In addition to a broad cognizance of compaction control procedures in the mass files, the principal author personally participated in the preparation of three reports treating in detail the compaction control achieved on several USACE dams which were designated at random by Headquarters USACE (Torrey 1970a; Torrey 1970b; and Strohm and Torrey 1982). Torrey (1970c) also reported compaction control results for other USACE dams. All of these projects were constructed before the current era of contractor quality control and government quality assurance, i.e., government forces were totally responsible for all aspects of quality control. These project data confirmed that excellent compaction control practices were the rule but there were some exceptions. The exceptions were pretty much a balance between cases of obviously erroneous procedure and those where the compaction control method was 
not precise enough to actually control the compaction in light of specified ranges or values. A case of obvious mistake is one dam embankment in which the entire chimney drain sand fill density data set indicated relative densities in excess of 300 percent. Fill relative densities in excess of 100 percent but less than, say, 120 percent are not unusual simply because there is no reason to expect that the laboratory method for determining maximum density (USACE 1970, Appendix XII) corresponds to the densities attainable by the field compaction equipment. The reported relative densities of 300 percent could have been the result of incorrect values of maximum and minimum densities for the chimney drain materials, simple computational errors, poor calibration of the sand used in the sandcone fill density device, or even poor calibration of field laboratory balances. A case of inadequate precision of control method is that for a dam where there was a correlation among maximum dry density, optimum water content and Atterberg Limits. The correlation exhibited so much scatter that the line of best fit could not possibly have predicted the desired values with sufficient precision. However, in every case where there was some reason to question the adequacy of the control procedure used, there was no question as to the adequacy of the number or quality of Record Samples taken from that embankment or concerning the satisfactory results of tests performed on those samples relative to design requirements.

24. The most popular method within the USACE for estimating the maximum dry density and optimum water content of a fraction of the total material or a scalped/replaced derived gradation has been by either the one- or two-point field laboratory compaction method. With the exception of the USBR Rapid Method (ASTM 1991e and USBR 1989b), the other methods given in USACE (1977), i.e., correlations with Atterberg limits or visual comparison have seldom been proved to be sufficiently reliable as will be discussed below. The USBR Rapid Method has been used very little by the USACE for no reason the authors can see other than its complexity for a novice user. Technical objection to the Rapid Method has been raised by some in the past because it is based on forcing a parabola-shaped compaction curve through three compaction points which may not be true to the actual shape of the material's compaction curve. However, to the authors' knowledge, no one has documented the extent of any potential error this may produce. The advantage of this method lies in the fact that it yields values of percent compaction and deviation of fill water content from optimum water content without the time delay associated with 
obtaining oven-dried water contents. The USBR Rapid Method is very popular in the private sector.

One- and Two-Point Compaction Methods

25. Detailed description of the one- and two-point methods is provided in Figures 11 and 12, respectively, as taken directly from EM 1110-2-1911, Appendix B. The two-point method has been most frequently used because it adds a degree of confidence in the estimation of the maximum dry density and optimum water content associated with the fill sample as compared with the one-point approach. The most obvious necessity for use of these two methods is the successful development of a family of compaction curves representing the soil being placed in the fill which sufficiently defines a "line (or curve) of optimums," such as that illustrated in Figures 11 and 12 . Given that the soil in a borrow area visually appears to be very consistent with respect to color and classification, it can be considered typical that compaction curves for that soil (whether it contains gravel or not) may range over several $\mathrm{lb} / \mathrm{cu} \mathrm{ft}$ in maximum dry density and several percentage points in optimum water content. The development of several such families may be necessitated if a variety of suitable soils are encountered in the borrow areas. As a continuing check on the developed families of curves, full five-point compaction curves should be run periodically during construction on fill samples. If a variety of soils is employed, it becomes additionally necessary to be able to distinguish the family of compaction curves which is pertinent to any given fill sample. Obviously, the closer the "lines of optimums" for the different families of curves, the more accurate the means of distinguishing the appropriate family for a given fill sample must become. The condition in proximity and parallelism of two "lines of optimums" at which two families can be treated as one depends upon a judgment of the acceptable error in controlling compaction. It is a fortunate situation if factors so simple as difference in color or difference in borrow source permit the selection of the appropriate family for a fill sample. From there, it becomes increasingly difficult and time consuming if such identifiers as gradation, Atterberg limits, or specific gravity become involved. In the one-point compaction method (Figure 11), material from the fill density test (and additional material from the same location, if needed) is allowed to dry to a uniform water content (achieved by thorough mixing during drying) on the dry side of estimated optimum, and then compacted using the same equipment and procedure used to obtain 
the full five-point curves of the family of curves. The water content and dry density of the compacted sample are then used to estimate its optimum water content and maximum dry density by extrapolating a curve through the one compaction point in a manner approximately parallel to the dry "legs" of the adjacent five-point compaction curves of the family of curves to a point on the "line of optimums" as shown in Figure 11. In the two-point method, one sample from the location of the fill density test is compacted at the fill water content if thought to be on the dry side of optimum (otherwise, reduced by drying) and a second sample is dried back by an additional two to three percentage points compared with the first sample and compacted. The two compaction points thus obtained are then used as shown in Figure 12 to extrapolate an estimated optimum water content and maximum dry density for the sample. The requirement of compacting one or two points on the dry side of optimum derives from the fact that the dry portions of the curves of a family will tend to be separate, whereas on the wet side of optimum, the curves tend to merge together. One or two points on the wet side of optimum would likely fail to clearly indicate which curves of the family should be used to guide an extrapolation to the line of optimums. The authors are of the opinion that the one or two-point method can estimate optimum water content and maximum dry density for fill samples which are within the precision of the compaction test provided that the lines of optimums of the families of curves are neatly defined. That is to say that the estimated values will fall within the range of values obtained if a series of replicate five-point compaction curves were generated on the same material.

Correlations among optimum water content, maximum dry density and Atterberg limits

26. Another method for estimating optimum water content and maximum dry density associated with the fill density sample is by correlation of maximum dry density and optimum water content to Atterberg limits (usually Liquid Limit (LL) or Plasticity Index (PI)). Figure 13 shows actual project specific correlations of compaction parameters versus LL obtained during the design phase of a major USACE dam embankment. The equations of the solid straight lines of "best fit" shown in Figure 13 were determined using the method of least squares. The method of least squares is a mathematical means of placing a straight line (or curve) through scattered data such that the sum of the squares of the perpendicular distances from each data point to the line is a 
minimum. However, the least square line of "best fit" does not necessarily mean "good fit" or even that a straight line fits better than a curved one. To avoid as much complexity as possible in the discussion to follow, it will be assumed that a straight line has been determined as superior to any curved line as a fit to the data sets of Figure 13 and that statistical determination has been made that there is strong correlation between optimum water content and Atterberg limits and maximum dry density and Atterberg limits. These decisions can be made statistically in a relatively simple manner but will not be discussed herein.

27. The precision with which the linear correlations of Figure 13 can estimate values of optimum water content or maximum dry density from LL can be judged by reference to the statistical parameter called the "standard error of the estimate" for each case. It can be seen from Figure 13 that any single value of LL corresponds to a range in values of optimum water content or maximum dry density. If enough data points were obtained, the distributions of the values of optimum water content and maximum dry density over their ranges for any single value of LL could be determined. In order to translate from the simple mathematical fitting method of least squares to statistical inference concerning how well the line of best fit predicts individual values of the $y$-axis parameter (optimum water content or maximum dry density, Figure 13) from a given value of the $x$-axis parameter (LL, Figure 13), an assumption about the statistical distribution of the $y$-axis parameter for any given value of the $x$-axis parameter must be made. For a linear regression analysis, that assumption is typically that the $y$-axis parameter is normally distributed for a single given value of the $x$-axis parameter as shown in Figure 14 . This is equivalent to assuming that the values of the $y$-axis parameter vary in a random manner over its total possible range for any given value of the $x$-axis parameter. The mean of that normal distribution is equivalent to the value of the $y$-axis parameter on the line of best fit (Point B of Figure 14). The standard error of the estimate, $S_{y, x}$, of the $y$-axis parameter for a given value of the $x$-axis parameter (see Figure 14) is analogous to \pm one standard deviation of the normal distribution either side of the line of best fit (the equivalent mean of the distribution). The standard error of estimate is calculated in a manner analogous to the standard deviation. For instance, from Figure 13 it is seen for the correlation of optimum water content versus LL that the standard error in estimating a value of optimum water content from a 
value of LL is \pm 1.4 percentage points in water content. Furthermore, also from Figure 13, the standard error in predicting maximum dry density from a value of LL is \pm 2.7 pcf. For a normal distribution, about one-half of the data points fall within the range of $\pm 2 / 3$ of one standard deviation, $\sigma$, from the mean or, in this case, $\pm 2 / 3$ of one standard error of the estimate, $\mathrm{S}_{\mathbf{y}, \mathbf{x}}$. Also, about 95 percent of the values would be expected to fall within $\pm 2 S_{y . x}$ and about 99 percent within $\pm 3 \mathrm{~S}_{\mathrm{y} \cdot \mathrm{x}}$.

28. The practical impact of the above concepts is important to understand. In using the correlations of Figure 13, it would be expected that about one-half of the estimates of optimum water content and maximum dry density based on LL would vary from their correct values by more than $2 / 3$ of the respective $S_{y, x}$. Therefore, one-half the values of optimum water content estimated from the LL using the line of best fit of Figure 13 could be in error by more than \pm 0.9 percentage points. Similarly, about one-half the values of maximum dry density estimated from the LL using the line of best fit would be expected to be in error by more than $\pm 1.8 \mathrm{pcf}$. If a correlation is to be useful for compaction control, its precision in estimating optimum water content and maximum dry density should at least be equivalent to the precision of the laboratory compaction test in determining those values. It would seem most appropriate for a correlation that precision be stated in the form of the "difference $2 \sigma$ limits" or 2.77 , as previously defined in paragraph 12 . For the correlation, this becomes the "difference $2 \mathrm{~S}_{\mathrm{y}, \mathrm{x}} 1$ imits" or $2.77 \mathrm{~S}_{\mathbf{y}, \mathrm{x}}$. For instance, accept, for the sake of argument that the multioperator (same laboratory) difference $2 \sigma$ limits for determination of optimum water content by the five-point compaction test is \pm 3.0 percentage points. This level of multioperator precision is the rough average found by the RRL comparative study previously discussed (see Table 9). (The authors do not intend to sanction the multioperator precision reported by RRL by using them in this example since they are of the opinion that experienced technicians in the same laboratory should produce more consistent results). The multioperator case is used here because it is the likely manner in which compaction tests would be performed in a field compaction control laboratory. To match the RRL level of precision in determining optimum water content, the correlation with LL of Figure 13 would have to exhibit an $\mathrm{S}_{\mathrm{y}, \mathrm{x}}$ such that $\pm 2.77 \mathrm{~S}_{\mathrm{y}, \mathrm{x}}= \pm 3.0$ percentage points or $\mathrm{S}_{\mathbf{y}, \mathrm{x}}= \pm 1.1$ percentage points. That correlation does not meet that standard since it exhibits an $\mathrm{S}_{\mathrm{y} . \mathrm{x}}$ of \pm 1.4 percentage points. The RRL study 
found a multioperator difference 20 limit for maximum dry density to average about $\pm 4.0 \mathrm{pcf}$. To meet this precision, the correlation with LL of Figure 13 would have to meet the criterion $2.77 \mathrm{~S}_{\mathbf{y}, \mathbf{x}}= \pm 4.0$ or $\mathrm{S}_{\mathbf{y}, \mathbf{x}}= \pm 1.4 \mathrm{pcf}$. It is seen that the correlation of maximum dry density versuis LL of Figure 13 with an $S_{y . x}= \pm 2.7$ pcf falls considerably short of that standard. These examples using a pair of actual correlations is intended to emphasize that correlations should never be used without a formal assessment of their quality and regular updating with new data points during construction. It is certainly not adequate to just fit a line to a set of data and assume it to be an acceptable correlation.

\section{Visual comparison}

29. In the visual comparison method, selection of an appropriate compaction curve representing the fill sample is based on visual association of the material from the fill density test with material (usually jar samples) on which five-point compaction tests have been run. Since materials that appear similar may have widely varying compaction characteristics, this method is very rarely reliable. It should never be considered for use on major fills. USBR Rapid Compaction Method

30. This method (USBR 1989b) consists fundamentally of a three-point compaction test on the minus No. 4 fraction of the fill density sample such that the three points are at known relative increments of water content apart and include both the dry of optimum and wet of optimum legs of the compaction curve. One of the points is always at fill water content. The authors will attempt to give only an overview of the method and refer the reader to the cited reference above for details. A 7.5-1b specimen of the minus No. 4 fraction of the fill density sample at fill water content is the working basis for the method. The necessary compaction points are determined by adding or subtracting designated percentage point increments of water content referenced to the 7.5-1b specimen at fill water content. The actual value of fill water content is not needed to apply the procedure so that the added or subtracted water increment is a percentage of the 7.5-1b specimen. The actual fill water content is determined by oven drying for record purposes and as a check on the satisfactory results of the method but the usual day required to obtain that value is avoided in the method itself. The wet densities for the compaction points are converted to values relative to fill water content by dividing the value of the compacted wet density at fill water content by the value, one 
plus the added or subtracted water increment in percent. This is the form of the familiar equation for calculating wet density using dry density and water content but with the wet density at fill water content treated as if it were the dry density and the added or subtracted water increment in percent as if it were the water content. Once the three compaction points are obtained, a parabola is fitted through the points in a prescribed, simplified manner to represent the wet density compaction curve for the sample. The known relative spacing of the compaction points with respect to water content increment and converted wet density coupled with the geometry of a parabola permit the construction of tables which yield the position of the fill water content compaction point relative to that of the peak (apex) of the parabola. That relative position is equivalent to deviation of fill water content from optimum water content and percent compaction. At first glance, the method appears complex because of the alternate procedures which may be necessary to achieve the two points in addition to that at fill water content which are in the satisfactory positions on the compaction curve. However, that complexity disappears with familiarity with the objective and the specific straightforward procedures. 
PART IV: GENERAL EFFECTS OF GRAVEL ON THE MOISTURE-DENSITY CURVE

31. Before proceeding to discussions of laboratory and fill compaction control methods used to accommodate soils containing large particles, it is first appropriate to examine the impact of increasing gravel content on the moisture-density curve. This is best accomplished by drawing from the literature comparative compaction curves obtained by various investigators. It must be borne in mind throughout the discussion to follow that there is no information available which might suggest the precision of large scale compaction tests. It is also important to note that every investigation referred to below utilized a different version of large scale test.

32. In 1963, the USBR reported the results of a study on the compaction characteristics of soils containing gravel in varying amounts from zero to 50 percent by weight. The test gradations were generated by combining a lean clay soil from the vicinity of Twin Buttes Dam, Texas, with a subangular to subrounded sand and gravel from the vicinity of Yellowtail Dam, Montana. These two soils were combined in various proportions to produce the 10 research gradations shown in Figure 15. The maximum particle size tested was 3 in. (gradation 36R-1, Figure 15) and all other gradations shown in Figure 15 represent scalped fractions of that material. Standard Proctor and large-scale (standard effort) compaction tests for soils containing gravel were performed in accordance with test Designations E-11 and E-38, respectively, of the USBR Earth Manual, First Edition (1963a). The Standard Proctor test utilized a hand-held rammer in the 4.0-in. diam $(1 / 20 \mathrm{cu} \mathrm{ft}) \mathrm{com}$ paction mold. The large-scale test employed a mechanical compactor and a 20-in. diam by 11-in. high mold.

33. The compaction curves obtained by the USBR on the 10 test gradations are shown in Figure 16. Only the tests on gradations containing gravel and the minus No. 4 fraction (36R-1 through 36R-5) are pertinent to this discussion. The first noteworthy point about the curves of Figure 16 relates to the minus No. 4 fraction (gradation 36R-5). This gradation was tested in both the 4-in. mold and the 20-in. mold with strikingly different compaction curves obtained. The difference in maximum dry density between the large mold and the small mold was 9.0 pcf even though the total applied compaction effort was identical in foot-pounds per cubic foot, i.e., standard effort $(12,300 \mathrm{ft}$ $1 b / c u f t)$. This difference will be addressed again in discussion of 
compaction data obtained by Torrey and Donaghe (1991). While it cannot be said that the large mold test for gravelly soil yields results consistent with those for fractions containing no gravel obtained in the 4 -in. mold, it is seen from Figure 16 that the large mold tests taken as a group were consistent with optimum water content decreasing and maximum dry density increasing with the addition of increasing amounts of gravel. This was true up to the maximum tested gravel content of 50 percent. It will be seen below that this consistency does not hold true for all materials.

34. Gordon, Hammond and Miller (1964) report results of compaction studies conducted by the California Department of Water Resources (CDWR) on the gravelly soils selected for placement in the impervious core of Oroville Dam. The material was obtained from a borrow source known as the airport area in an alluvial deposit and was fairly well-graded over a gradation range of gravelly, clayey sand to clayey, sandy gravel containing up to 65 percent by weight of gravel sizes. The material gradings fell into four major groupings as shown in Figure 17. These groupings were treated by selecting representative samples as indicated in Table 10. Maximum particle sizes investigated were obtained by successive scalping of the minus 4-in. representative gradations and included 4-, 3-, 1-1/2- and 3/4-in. and minus No. 4 sieve fractions. The gradation curves for the tests indicated in Table 10 are shown in Figure 18. The compaction equipment summarized in Table 11 included a variety of mold diameters and hammer weights coupled with procedures to yield an intermediate (between standard and modified efforts) compactive effort of $20,000 \mathrm{ft}-1 \mathrm{~b}$ per cu ft (an apparent CDWR standardized test effort of the time). The CDWR standard procedure $(20,000 \mathrm{ft}-1 \mathrm{~b} / \mathrm{cu} \mathrm{ft})$ for compaction tests on minus No. 4 material is seen from Table 11 to have utilized a 4.24-in. diam mold.

35. The compaction curves obtained by Gordon, Hammond and Miller are shown in Figure 19. The significant separation of the curves for the minus No. 4 fractions from those for the gradations containing gravel may reflect the equipment size effects previously pointed out for the USBR data. It is most significant to observe from Figure 19 that a consistent trend of decreasing optimum water content and increasing maximum dry density with increasing gravel content does not occur for all the materials tested. In some cases, the compaction curve for the minus $4-i n$. gradation containing the highest gravel content exhibits a distinct shift in position toward a dryer optimum 
water content and lower maximum dry density compared to the trends established by the related scalped fractions of lower gravel contents. This occurred for one minus 4-in. gradation containing only 35 percent gravel (Grading A). It will be shown later that this effect is probably associated with gravel content and gradation and not maximum particle size in itself. Also, in most of the cases of Figure 19, the compaction curves for the highest gravel contents shows at least some distinct change in shape compared with the lesser gravel content fractions. So, it is inferred that increasing gravel content may result in a significant change in the manner in which applied compactive effort affects the material and that the gravel content at which that change begins to occur is dependent upon, among other factors, gradation. For the borrow materials of the Oroville Dam core, that gravel content was seen to be at least as low as 32 percent.

36. Donaghe and Townsend (1975) report a compaction test series on artificially blended gradations. The basic objective of their study was to assess the validity of the scalping with replacement procedure. The fullscale test gradations with a maximum particle size of 3 in. were generated by maintaining the percent clay (CL) fines (minus No. 200 sieve) constant at 25 percent while varying the sand and gravel contents to achieve mixtures with a gravel contents ranging from zero to 60 percent, as shown in Figure 20 . The associated scalped/replaced gradations with a maximum particle size of 3/4-in. are shown in Figure 21. Note that the only difference between a full-scale gradation and its associated scalped/replaced gradation is the maximum particle size and, therefore, the gradation of the gravel fraction. A scalped and replaced gradation contains the same gravel content as the parent full-scale gradation but the gravel fraction is more uniformly (more poorly) graded. For the purposes of this discussion, the scalped/replaced gradations may be viewed as just another set of gravelly soils with more uniform gravel fractions. Figure 20 shows the compaction curves obtained for the full-scale gradations and Figure 21 shows those obtained for the associated scalped/replaced gradations. Table 12 lists data pertinent to mold sizes, rammer sizes, and the compaction procedures they employed. Table 13 summarizes the results of the testing. Figure 20 reveals that the full-scale gradations containing 50 and 60 percent gravel exhibited compaction curves of significantly different shape compared with the other full-scale gradations, with increasingly dryer optimum water contents and declining maximum dry density. Figure 21 also shows the 
same occurrence for the minus 3/4-in. gradations but the significant shift in the position of the compaction curve is also evident at the lower gravel content of 40 percent as compared to 50 percent for the full-scale material. With respect to maximum dry density, these gradations exhibited a clear "optimum" gravel content. Note that all the full-scale gradations and all the scalped/replaced gradations had the same maximum particle sizes, respectively, which supports the suggestion made earlier concerning the data of Gordon, Hammond and Miller (1964) that a shift in compaction curve position with higher gravel content is a function of gradation rather than maximum particle size in itself. And, Figures 20 and 21 suggest that the gravel content at which the value of maximum dry density will reach a peak value (optimum gravel content) may be expected to decline as the uniformity of the gravel fraction increases for cases where the minus No. 4 fraction is not a variable. Surely, the gradation of the minus No. 4 fraction and the plasticity of fines also play a role so that in the general sense, the gradation of the gravel fraction is not the whole story.

37. In 1985, Garga and Madureira reported a very extensive compaction testing program related to the construction of Sao Simao Dam in Brazil. The soils varied from well-graded gravelly clayey sands to clayey sandy gravels. For the testing program, Garga and Madureira idealized the shape and range in the natural gradàtions, as indicated in Figure 22, where they envision the gravel fractions and minus No. 4 fractions as linearly graded in the semi-log plot coordinates. They reconstituted many test gradations with various gravel contents in this fashion and utilized various sized molds fundamentally after the test procedures E-11 and E-38 of the old USBR Earth Manual (1963a) as previously referenced. Results of their program are summarized in Table 14. In the interest of brevity and sufficient for the purposes here, Figure 23 synopsizes the effects on maximum dry density of increasing gravel content. It is seen from Figure 23 that some gradations appeared to reach an "optimum" gravel content somewhere above 60 percent gravel while others did not. It is also seen that for the minus 3-in. gradations, an "optimum" gravel content appeared to have occurred under modified effort but not under an effort intermediate to standard and modified. It is logical that compactive effort must also be included with gradation as influencing the gravel content at which the compaction curve may shift to the drier side and exhibit a lower maximum dry density as compared with gradations containing less gravel. For a given 
gradation, increasing compactive effort will translate to increasing maximum dry density and decreasing optimum water content. Although for different reasons, this is the same effect seen by increasing gravel content for a given compactive effort. If the two effects are combined, i.e., increasing gravel content and increasing compaction effort, it isn't surprising if "optimum" gravel content decreases.

38. Torrey and Donaghe (1991) tested the artificially blended gradations shown in Figures 24 and 25 and described in Table 15. One test suite was performed with all the gradations of Figures 24 and 25 containing clay $(\mathrm{CH})$ fines and a second suite was performed with all the gradations reconstituted with non-plastic silt (ML) fines. Note from Figures 24 and 25 that each family of gradation curves was generated by successive scalping of a minus 3-in. gradation. The comparative trends in maximum dry densities and optimum water contents are shown in Figures 26 through 29 for both categories of fines. It is interesting to note the effects of plasticity of fines from Figures 26 through 29. While the compaction curves for gradations containing clay $(\mathrm{CH})$ fines show a significant decline in maximum dry density and optimum water content beginning at a gravel content in the minus 3 -in. gradation of 52 percent and above, the identical gradations containing silt (ML) fines did not. For those gradations containing silt fines, the maximum dry density continued to increase up to the maximum gravel content in the minus 3 -in. gradation of 64 percent.

39. The overview of the literature presented above reveals that the consistency of the trend in maximum dry density and optimum water content with increasing gravel content is a function of compactive effort, gradation and plasticity of fines. There may be other effective variables as well. The most important insight to be gained from the observed cases of occurrence of an "optimum" gravel content above which the maximum dry density reverses its increasing trend is relative to compaction control procedures based on a fraction of the total material such as the minus No. 4 fraction. As long as the maximum dry density and optimum water content of the total material with increasing gravel content lie along a consistent trend which includes the values for the fraction, it may be possible to predict the parameters for the total material from those of the fraction by some single equation or correlation. However, when the compaction parameters for the total material begin to significantly deviate from that consistent trend, it should be expected that 
difficulties will arise in predictions based on the fraction. 


\section{General}

40. This section will discuss the various means that have been used by various agencies to avoid compaction testing of full-scale gradations containing appreciable gravel with a maximum particle size in excess of $3 / 4-i n$. of course, minus $3 / 4-i n$. materials present no particular problem in that compaction tests using the 6 -in. diam mold are routine. The avoidance procedures for gravelly soils with larger particles were not adopted because it was not possible to perform larger diameter mold compaction tests. The reason is that such large tests require large and heavy equipment of several types, considerable working space, large amounts of material, and are time consuming and labor intensive. These requirements can be accommodated for relatively limited design testing programs conducted in central major laboratory facilities, but are difficult to use, if not often impractical, in a construction control environment. While it is true that the Corps of Engineers adopted a 12-in. diam mold compaction test for earth-rock mixtures (USACE 1970, Appendix VIA), it has never been used without considerable complaint for the reasons cited above.

\section{Testing of Altered Gradations Perceived as Simulations of the Full-Scale Gradations}

\section{Scalping}

41. The most obvious possible means of eliminating the testing problems associated with large particles is to remove them from the total material and test the resulting fraction. For most conventional soils laboratories, this would mean removal of plus 3/4-in. sizes and compaction testing in the 6-in. diam mold. Or, it may translate to removal of the plus No. 4 sieve sizes, i.e., all the gravel, and testing in the 4-in. diam mold. Or, in the case of EM 1110-2-1906, Appendix VIA, it would mean removal of up to 5 percent by weight of plus $2-i n$. sizes. This immediately raises the question of how much oversized (plus No. 4, plus 3/4-in. or plus 2-in. material) can be scalped, if any, before the compaction parameters obtained on the fraction deviate too greatly from those of the total material. That question has been argued for 
years and usually in the presence of a dearth of actual comparative test data to support the various opinions. EM 1110-2-1906, Appendix VI, permits the removal (scalping) of up to 5 percent of the total material by weight of either plus No. 4 or plus $3 / 4-i n$. sizes for tests performed in the $4.0-i n$. and 6.0-in. molds, respectively. However, Appendix VIA states that the 12-in. mold test for earth-rock mixtures should be used for materials containing particles larger than the $3 / 4-i n$. sieve size if those particles exceed 10 percent by weight of the total material. This leaves a question as to what to do for materials which contain between 5 and 10 percent plus $3 / 4-i n$. sieve sizes. This was obviously an oversight in the last revision of the manual. The 10 percent figure stated in Appendix VIA should have also been changed to 5 percent to be consistent. None of this is to say that even 5 percent scalping is generally acceptable. When the attempt is made to track down the USACE former 10 percent scalping standard, it is found that it came about as a consensus among Division Laboratories which was not specifically based on comparative testing of full-scale versus scalped gradations. The more recent reduction of the allowable scalping to 5 percent was again a judgment call, but on the basis of some data showing that 10 percent scalping may produce compaction data on the fraction which is unacceptably different from that for the total material.

42. Torrey and Donaghe (1991) addressed the question of maximum permis sible degree of scalping. Figures 24 and 25 show their test gradations which were duplicated to contain both nonplastic silt (ML) and clay (CH) minus No. 200 sieve size fractions. Table 15 provides a descriptive summary of the test gradations. Figures 26 through 29 show the trends in maximum dry density versus optimum water content obtained from the compaction tests performed on the test gradations. It was previously pointed out that the program was based on successive scalping of four full-scale gradations with a maximum particle size of 3 -in. and variable gravel content. Figures 30 through 33 present Torrey and Donaghe's interpretation of the data obtained in terms of maximum permissible degree of scalping. The maximum permissible degree of scalping was based on a precision of \pm 2.0 percent of the mean value in estimating maximum dry density from the scalped fraction and on 10.0 percent of mean value in estimating optimum water content from the scalped fraction. For comparative purposes, the 5 percent scalping rule of EM 1110-2-1906 is shown in each of the Figures 30 through 33. These figures reveal a trend which is 
logical but, in a sense, opposite to the apparent prevailing presumptions about scalping. Torrey and Donaghe considered the data points widely deviant from the indicated trend to be the result of precision in their compaction tests. They had performed all of the compaction testing and fitted compaction curves to the data before entering into any comparative analyses. They had decided that the values of optimum water content and maximum dry density obtained from that initial fitting of compaction curves would not thereafter be adjusted in any manner to improve the appearances of any observed trends. Perfectly reasonable adjustments could have been made in fitting the compaction curves which would have moved the widely scattered points of Figures 30 through 33 more into alignment with the other data. For both optimum water content and maximum dry density, Figures 30 through 33 imply that the more gravel present in the gradation, the greater the quantity which may be scalped without severely altering the values of optimum water content and maximum dry density. Or, conversely, the less gravel in the gradation, the smaller the amount which can be scalped without significant impact on the compaction parameters. This is opposite to the prevailing assumption that a small amount ( 5 percent or less) of oversized material present in a gradation can be discarded. Note in Figures 30 through 33, that 5 percent scalping produces significantly different compaction parameters for any materials tested by Torrey and Donaghe which contained less than 15 to 20 percent gravel. There was no case where 5 percent scalping was acceptable within the designated precisions for a material which contained 5 percent or less gravel. In summary, the addition of very small quantities of gravel to a minus No. 4 sieve size material produces very significant changes in the compaction curve. Optimum water content decreases and maximum dry density increases. As the amount of added gravel increases, the rate of change of the compaction parameters decreases. So, the current scalping practice is apparently being applied to gradations which are the most sensitive to scalping. These results leave the question of acceptable degree of scalping in the same ambiguous position as is that of precision of the compaction test. The authors leave the subject warning that for some materials, 5 percent scalping will result in optimum water content of the fraction which is more than one percentage point higher than that of the parent full-scale material and maximum dry density which is more than 3 pcf lower. It is probably a good rule to suspect the validity of 5 percent scalping for any material containing less than 20 percent gravel. 


\section{Scalping with replacement}

43. The procedure of scalping and replacing was envisioned as a method of modelling the full-scale gradation. The method is explained as follows. Assume a material contains too much plus $3 / 4-i n$. sieve size to be simply scalped and discarded, i.e., more than 5 percent. The plus $3 / 4$-in. material would first be scalped and then replaced with an equal weight of the material passing the $3 / 4-i n$. sieve but retained on the No. 4 sieve. The gradation of the replacement material is required to have the same gradation as the No. 4 to 3/4-in. sieve size range in the full-scale unaltered gradation. So, scalping with replacement does not alter the percent gravel but it reduces the maximum particle size and produces a more uniform (less well-graded) gravel fraction. It, as well as scalping, has been practiced not only for compaction tests but also for preparation of material for triaxial shear testing. As has been the case for degree of scalping, the acceptance of the scalping with replacement procedure has been in the absence of comparative test data proving its acceptability. In recent times, the scalping and replacement procedure has been documented by several investigators to yield a different material and not a compaction property or shear strength "model" of the parent full-scale gradation. ASTM standards no longer permit the use of the method. Before EM 1110-2-1906 was revised to include Appendix VIA, i.e., the 12-in. diam mold compaction test for earth-rock mixtures, the scalping and replacement procedure followed by compaction in the 6-in. diam mold was allowed for soils containing more than 10 percent plus $3 / 4$-in. sieve sizes. With the addition of the larger mold procedure, the manual now requires use of the 12 -in. mold test for soils containing more than 5 percent plus $3 / 4-i n$. sizes. Scalping and replacement is allowed only for the case where the material contains more than 10 percent (should state 5 percent) by weight retained on the 2 -in. sieve.

44. Donaghe and Townsend (1975) produced comparative compaction data which illustrate the typical effects of the scalping with replacement procedure. Figure 34 compares maximum dry densities obtained in the 18-in. diam mold on seven minus 3 -in. full-scale gradations with maximum dry densities obtained on corresponding minus $3 / 4-i n$. sieve scalped/replaced gradations obtained in the 6 -in. diam mold. It is easy to see from Figure 34 that the scalped/replaced gradations did not model the full-scale gradations with respect to maximum dry density. Figure 35 shows that the scalped/replaced gradations increasingly underestimated maximum dry density of the full-scale 
materials with increasing gravel content. If the scalping with replacement procedure based on the 3/4-in. sieve were required to meet the precision of the full-scale test in the 18-in. mold of, say, two percent of mean value, Figure 35 shows that this would not be true for any gradation containing more than about 15 percent gravel. Of course, if the scalping/replacement had been based on the 2-in. sieve and the compaction performed in the 12-in. diam mold, the differences would have been very much smaller and perhaps acceptable simply because less material would have been scalped and replaced. However, it must be remembered that the subject of this section is procedures employed to completely avoid large scale testing. This was the original objective in using scalping with replacement.

45. Figures 36 shows the differences between the optimum water contents of the full-scale gradations and those obtained on companion minus 3/4-in. sieve scalped and replaced gradations for the six gradations tested by Donaghe and Townsend (1975) which contained minus No. 4 sieve fractions. Soils which do not differ from one another significantly with respect to the specific gravity of solids, whether $G_{s}$ for minus No. 4 gradations or the weighted specific gravity for gravelly gradations (see EM 1110-2-1906, Appendix IV), tend to conform to a trend in maximum dry density and optimum water content. That trend is that the higher the maximum dry density, the lower the optimum water content. The differences in optimum water contents indicated in Figure 36 and plotted in Figure 37, conform to that trend. The maximum dry densities of the scalped and replaced gradations are lower than those of the corresponding full-scale gradations, and'so the optimum water contents of the scalped and replaced gradations are higher. Just as the differences in maximum dry densities between full-scale and scalped/replaced gradations tended to increase with gravel content so also do the differences in optimum water contents. Those differences in optimum water content begin at the already significant level of just over one percentage point at only 10 percent gravel.

46. In the compaction control process, the compaction parameters obtained on scalped/replaced gradations are assumed to be equivalent to those of the full-scale materials. That assumption is not correct and results in values of fill percent compaction and deviations of fill water content from optimum which are also incorrect. Based on Donaghe and Townsend's results, the extent of error would reflect the gravel content. The higher the gravel content, the larger the error in both fill percent compaction and deviation of 
fill water content from optimum as referenced to scalped/replaced values of maximum dry density and optimum water content, respectively. The reliance on scalped and replaced gradations will result in some degree of generally inflated values of fill percent compaction and deviations of fill water content from optimum which are generally wetter with respect to optimum than indicated by the scalped and replaced values. Assume for purposes of example that Figures 34 and 36 represent the full-scale and scalped/replaced maximum dry densities and optimum water contents, respectively, for materials being placed in an embankment. Furthermore, assume that a fill density sample contains 40 percent gravel, exhibits a fill density of $125 \mathrm{pcf}$, and a fill water content of 9.0 percent. Figure 34 indicates that the full-scale value of maximum dry density for that sample would be about 137.5 pcf and the scalped/replaced value would be about 132 pcf. Figure 36 shows that the fullscale optimum water content would be about 6 percent and the scalped/replaced optimum water content would be about 8 percent. Using the scalped/replaced values, the fill percent compaction would be calculated to be about 95 percent and the deviation of fill water content from optimum would be recorded as 1.0 percentage points on the wet side. However, the actual fill percent compaction would be 91 percent and the actual deviation of fill water content from optimum would be 3.0 percentage points to the wet side. If the specifications were specifically written around scalped/replaced gradations, there would be no problem. This would assume that during the design phase, any differences in engineering properties between full-scale and scalped/replaced materials had been addressed and it was found to be the case that the specifications based on scalped and replaced gradations would assure acceptable properties of the full-scale materials. However, if the specifications are intended to relate to placement of the full-scale materials, a real problem arises. The errors indicated by the above example are very significant in consideration of the typical values of desired percent compaction and specified ranges in placement water content relative to optimum. In fact, compaction of the material cited in the example would not be under control employing scalped/ replaced procedures. As has been emphasized before, the details of a compaction control procedure are not the whole story. Undisturbed samples taken from the fill for testing (Record Samples) may verify that it is of satisfactory quality. That does not ameliorate the waste of effort and money represented by an ineffective compaction control procedure or the potential 
difficulties in defending practice should some legal challenge arise.

47. If curves relating differences between full-scale gradation and scalped/replaced gradation compaction parameters versus gravel content such as those of Figures 35 and 37 can be developed during design, they could be used to correct scalped/replaced results in the fill compaction control procedure.

The idea of correcting values of maximum dry density and optimum water content obtained in a small mold on some fraction of the full-scale gradation to obtain values for the full-scale gradation has been around for some time. The converse of that idea, i.e., the correcting of fill density and water content for the full-scale material to obtain the density and water content of some fraction, has also been employed. It is obvious that if corrections can be applied to some fraction or full-scale results corrected to those of a fraction, it would be inefficient to develop corrections to scalped/replaced data because the scalping and replacement procedure is more complicated and time consuming than scalping. In fact, correction procedures for fractions have been derived and employed rather widely in compaction control of gravelly soils. The next section will address and assess those methods.

\section{Correcting Maximum Dry Density and Optimum Water Content To Account for the Effects of Gravel}

\section{USACE (1977). Engineer Manual 1110-2-1911}

48. With respect to correcting fill density test results for the total material to obtain the corresponding dry density and water content of some fraction, the USACE has often employed the equations given in Engineer Manual (EM) 1110-2-1911, Appendix B, which are as follows:

For correcting dry density of the total material to obtain that of a fraction:

$$
\gamma_{f}=\frac{f \gamma_{t} \gamma_{w} G_{m}}{\gamma_{w} G_{m}-C \gamma t}
$$


where

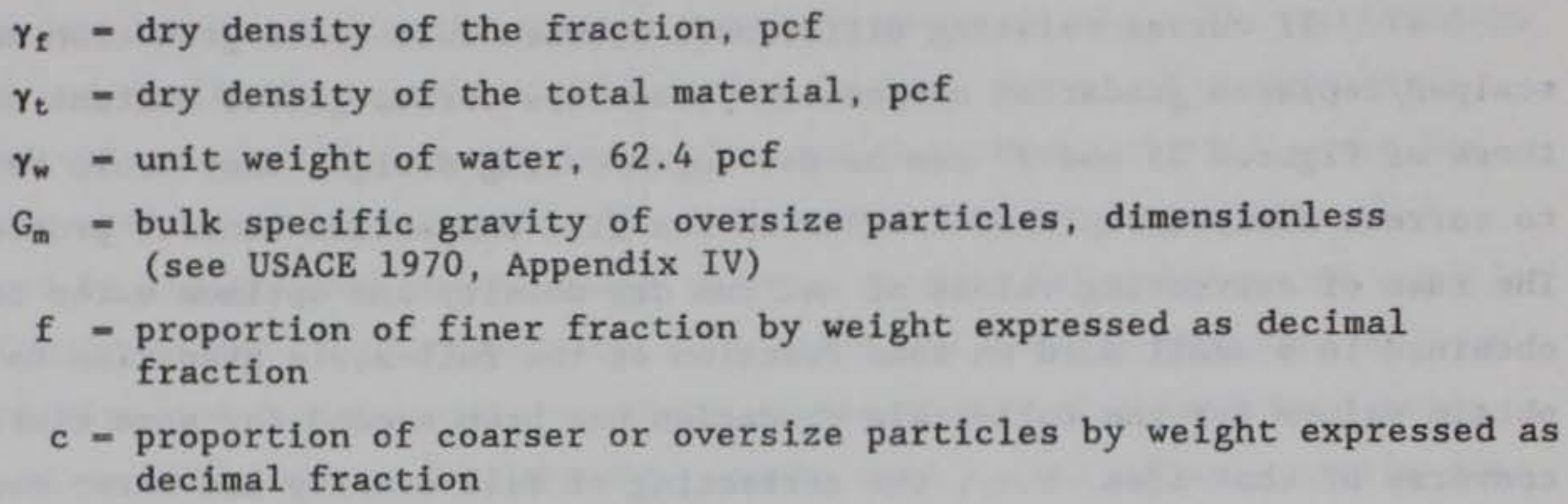

For correcting water content of the total material to obtain that of a fraction:

$$
W_{f}=\frac{W_{t}-C W_{c}}{f}
$$

where

$$
\begin{aligned}
& \mathrm{W}_{\mathrm{f}} \text { - water content of finer fraction, percent } \\
& \mathrm{W}_{\mathrm{t}} \text { - water content of total material, percent } \\
& \mathrm{W}_{\mathrm{c}} \text { - water content of coarser (oversized) fraction, percent, which is } \\
& \quad \text { taken as the absorption, A, of the gravel in EM 1110-2-1911 } \\
& \mathrm{f}, \mathrm{c}-\text { as defined for Equation } 1 \text { above } \\
& 49 . \quad \text { Equation } 1 \text { was originally derived by Ziegler (1948). The manual }
\end{aligned}
$$
states that the usefulness of the density correction Equation 1 is limited to cases where the proportion of oversize material is not greater than about 35 percent by weight. The reason for that limitation actually applies to a modified version of Equation 1 which will be clarified later in this section. Also note that Equation 2 differs from the version stated in EM 1110-2-1911 in that the manual shows the absorption of the gravel as the water content of the oversized material. Equation 2 is the correct general form of the relationship and must be true if the actual water contents of the two fractions are entered. A brief discussion of the effects of using the absorption as the water content of the oversize fraction will also be provided later in this section. The forms of Equations 1 and 2 are intended for use in correcting the fill dry density and water content of the total material (obtained from the fill density test) to obtain those values for a fraction which are then compared with the maximum dry density and optimum water content of that 
fraction to assess compliance with specifications which are referenced to the compacted state of that fraction. The maximum dry density and optimum water content of the fraction are obtained in a convenient smaller mold such as the 4-in. mold for the minus No. 4 sieve fraction or the 6 -in. mold for the minus 3/4-in. sieve fraction or may be estimated using the one-or two-point compaction methods previously described. The compaction specifications for the fraction should have been derived on the basis of assuring satisfactory properties of the full-scale material. However, when compaction specifications have been based on a fraction, it has been typical that no large scale tests of total materials have been conducted to verify that the specifications on the fraction assure equal or better qualities of the total material.

50. It has also been the practice to use rearranged versions of Equations 1 and 2 to predict the maximum dry density and optimum water content of the total material by substituting values of maximum dry density and optimum water content for a fraction, respectively, and then compare the fill dry density and water content directly to the predicted full-scale values. The maximum dry density and optimum water content of the fraction are either obtained in the 4- or 6-in. mold or estimated by the one- or two-point compaction test method. In this case, the specifications must be written concerning the compacted state of the total material. Again, specifications written around the total material may have been adopted on the basis of testing of either the minus $3 / 4-i n$. or minus No. 4 fractions during the design phase and on the assumption that satisfactory states of compaction of those fractions could be directly translatèd to the total material. Some discussion of this practice will be given later. The attempt to use the concepts of Equations 1 and 2 to predict maximum dry density and optimum water content of the total material first requires that their terms be rearranged to obtain dry density and water content of a total material from the values for a fraction. It is convenient for the discussions to follow to use these rearranged versions of the equations. The rearranged version of Equation 1 for estimating dry density of the total material from that of a fraction is as follows:

$$
\gamma_{t}=\frac{\gamma_{f} \gamma_{w} G_{m}}{f \gamma_{w} G_{m}+C \gamma_{f}}
$$


The rearranged version of Equation 2 used for calculating the water content of the total material from that of a fraction becomes:

$$
W_{t}=f W_{f}+C W_{c}
$$

When Equation la is used to predict the maximum dry density of the total material by entering the maximum dry density of a fraction, Equation 1 a is converted to:

$$
\gamma_{\mathrm{tmax}}=\frac{\gamma_{\mathrm{fmax}} \gamma_{\mathrm{w}} G_{\mathrm{m}}}{f \gamma_{\mathrm{w}} G_{\mathrm{m}}+C \gamma_{\mathrm{fmax}}}
$$

When Equation $2 \mathrm{a}$ is used to predict the optimum water content of the total material by entering the optimum water content of a fraction, Equation $2 \mathrm{a}$ is converted to:

$$
\mathrm{W}_{\mathrm{topt}} \approx \mathrm{fW}_{\mathrm{fopt}}+\mathrm{CW}_{\mathrm{c}}
$$

51. Equation 1 is a straightforward weight-volume relationship easily derived by association of all variable volume of voids with the fine fraction. The variable volume of voids is the volume of voids which changes with densification of the material. Therefore, the equation simply divides the material into two fractions, accounting for the contribution to dry density of each. Fundamentally then, its accuracy is contingent upon the condition that the finer fraction must completely fill the voids between the larger particles. In other words, there must be no extraordinary voids representing discontinuities in the mixture. This condition upon the accuracy of Equation 1 has proved to be generally unrestrictive in practice because maximum gravel contents usually encountered (say, less than 60 percent) do not result in partially filled voids among the gravel particles. This same condition must be met for Equation 3 but there is a second condition required as well because of the modification to obtain the maximum dry density of the total material. The second condition on accuracy of Equation 3 represents the weakness of the 
use of that equation to predict the maximum dry density of the total material from the maximum dry density of a fraction. This is tantamount to assuming that the increase in maximum dry density of the total material with increasing gravel content results only from the addition of gravel. weight of solids. In reality, as will be shown later in this report, the dry density of the finer fraction in the total material when that total material is at its maximum dry density is affected by the added gravel. The effect is such that the dry density of the fraction may be greater than or less than its maximum value depending on the material and its gravel content. Thus, use of Equation 3 may result in either under-prediction (at lower gravel content) or over-prediction (at higher gravel content) of maximum dry density of the total material. This is the reason that an "approximately equal" symbol is shown in Equation 3.

For the case of use of the minus No. 4 fraction as the finer fraction in Equation 3 , the gravel content above which use of its maximum dry density will begin to seriously over-predict the maximum dry density of the total material has been empirically estimated to be about 35 percent. Hence, the aforementioned restriction on use of the equation is stated in USACE (1977). Unfortunately, under-prediction of the maximum dry density was never addressed but may be significant as will be shown later. Furthermore, soils have been encountered for which use of the maximum dry density of the minus No. 4 fraction with Equation 3 significantly over-predicts the maximum dry density of the total material at gravel contents less than 35 percent. There is nothing to preclude use of the dry density of the minus

3/4-in. fraction with Equation 3. This alternative will also be addressed in this report.

52. It is important to emphasize that Equation 3 is only an approximate expression where the equal sign must be replaced with an "approximately equal" symbol and very careful restrictions placed on the range in gravel content over which that approximation is acceptably accurate. So, Equation 3 is no longer Ziegler's equation and is not a true weight-volume relationship except at some singular value of gravel content where the fraction happens to exist at its maximum dry density when the total material is at its maximum dry density.

53. Just as there is no reason to expect the maximum dry density of a fraction entered into Equation 3 to yield the maximum dry density of the total material, there is no reason to expect the optimum water content of a fraction 
entered into Equation 4 to yield the optimum water content of the total material. When the compaction curves for a total material and a fraction thereof are compared, the optimum water content of the total material will always be dryer than that of the fraction. If the optimum water content of the fraction entered into equation 4 is to generally yield the optimum water content of the total material, then the water content of the oversized gravel must always be precisely such as to account for the difference in optimum water contents between the fraction and the total material. This is not true as will be shown later and the water content of the fraction must be altered to produce optimum water content (i.e., 100 percent compaction) of the total material. That is to say, the fraction is generally not at its optimum water content when the total material is except for a singular value of gravel content in the total material. Therefore, Equation 4 must also be written with the approximately equal symbol and restrictions placed on range in gravel content over which the approximation is acceptably accurate.

54. The full-scale and associated minus 3/4-in. sieve scalped/replaced data obtained by Donaghe and Townsend (1975) can be used to illustrate the potential errors introduced by using the maximum dry density of the minus No. 4 fraction with Equation 3 in attempting to predict a value of maximum dry density for the total material. Figure 38 shows the results of such predictions for the full-scale materials. The values of total material maximum dry density predicted from those of the minus No. 4 fractions reasonably track the actual values up to a gravel content in the total material of about 35 percent. However, it is to be noted that the total materials were compacted in the 18-in. diam mold by mechanical compactor while the minus No. 4 fractions were compacted in the 4-in. diam mold with a hand-held rammer. These two test procedures would not yield identical compaction parameters for the same minus No. 4 material. Therefore, mold size and procedural effects played a significant role in the adequacy of predicted values up to 35 percent gravel content. 55. Figure 39 is companion to Figure 38 and shows the same comparative data relative to minus $3 / 4-i n$. sieve scalped/replaced gradations derived from the full-scale materials shown in Figure 38. Between Figures 38 and 39 the minus No. 4 fractions are the same and the respective percentages of gravel are the same. So, a scalped/replaced gradation can be viewed as just another material with all characteristics identical other than a more uniformly graded gravel fraction. Also, all the scalped/replaced data of Figure 39 were 
obtained in the 6 -in. mold with a hand-held rammer so that at zero gravel content there is no appreciable difference in the maximum dry densities between the 6-in. mold and the 4-in. mold. It has been fairly well established that compaction tests performed on a minus No. 4 material in both the 4 - and 6 -in. diameter molds can be taken as equivalent. Figure 39 shows that the predicted values of the maximum dry density of the scalped/replaced gradations were consistently and significantly higher than the actual values.

56. Since the minus No. 4 fractions between Figures 38 and 39 are the same and also the respective gravel contents, it is evident that Equation 3 may adequately predict the maximum dry densities for one family of gradations up to about 35 percent gravel (full-scale materials, Figure 38) but not perform satisfactorily at all for another family of gradations (scalped/replaced, Figure 39). It was pointed out earlier that scalped/replaced gradations will not generally replicate the maximum dry densities of parent full-scale materials. Therefore, the common minus No. 4 fraction data of Figures 38 and 39 cannot be used with Equation 3 to adequately predict both gradations' maximum dry densities for any gravel content.

57. Figures 40 and 41 show the results of applying Equation 4 to Donaghe and Townsend's data to predict the optimum water content of the fullscale and associated minus 3/4-in. sieve scalped/replaced gradations, respectively. In these cases, the absorption of the gravel was employed as the water content of the oversized fraction. Figure 40 shows that use of the optimum water content of the minus No. 4 fraction and the absorption of the gravel in Equation 4 results in an overprediction of the optimum water content of the full-scale material of about one percentage point up to about 35 percent gravel in the total material. Above about 35 percent gravel content, the values predicted using the minus No. 4 fraction are in good agreement with the actual values. The trend in quality of predicted values of optimum water content of the full-scale materials based on the minus No. 4 fraction is opposite from that seen in Figure 38 for maximum dry density. The predicted values for maximum dry density were relatively satisfactory up to about 35 percent gravel, whereas the predictions for optimum water content were best above that gravel content.

58. Figure 41 shows the results of attempted predictions of optimum water content based on the minus No. 4 fraction and the absorption of the gravel compared to the actual values for minus $3 / 4-i n$. sieve scalped/replaced 
gradations derived from the full-scale gradations of Figure 40 . It is seen from Figure 40 that the predicted values were consistently lower and within about one percent of the actual values of optimum water content up to only about 20 percent gravel. The deviation of predicted values from actual values steadily increased with increasing gravel content. Just as was the case for maximum dry density, the single set of predicted values resulting from common minus No. 4 fractions between the full-scale and scalped/replaced gradations does not suffice for both families of gradation curves. The predicted values of optimum water content generally trended closest to the full-scale gradations' actual values and deviated more seriously from the actual values for the scalped and replaced gradations.

59. Torrey and Donaghe (1991) also developed data useful for indicating the applicability of Equations 3 and 4 in predicting compaction parameters for total materials from those obtained on a fraction. Since their testing program was based on successively scalped fractions of minus 3 -in. full-scale gradations (see Figures 24 and 25 ), it is possible to judge the performance of Equations 3 and 4 by entering the compaction parameters of both the minus No. 4 and minus $3 / 4-i n$. sieve fractions. Figures 42 and 43 summarize the results of predictions (Equation 3) of maximum dry densities of the full-scale gradations from those of the minus No. 4 and minus $3 / 4-i n$. sieve fractions, respectively. These results are expressed as the differences between predicted values and actual values. A precision range of approximately two percent of the mean value of maximum dry density is also shown in the two figures for reference. Figures 44 and 45 show data in a similar manner for predictions (Equation 4) of optimum water contents of the full-scale gradations from those of the minus No. 4 and minus $3 / 4$-in. sieve fractions, respectively. The reference precision range shown in Figures 44 and 45 is approximately 10 percent of the mean value of optimum water content. The water content of the oversize fraction entered into Equation 4 in each case was the air-dry value for the grave1 ( 0.6 percent) as it was added to wetted minus No. 4 fractions in creating the compaction test specimens. Some error may result from this practice since water may have been exchanged between the two fractions during curing of the mixes before performance of the compaction tests.

60. Figures 42 and 43 show that predictions of maximum dry densities of the total materials based on the maximum dry densities of either the minus No. 4 or minus $3 / 4-i n$. fractions generally trend from less than to greater 
than the actual values regardless of plasticity of the minus No. 200 sieve fraction. This is the same trend seen previously in Figure 38 for the Donaghe and Townsend (1975) data. It is immediately evident in comparing Figures 42 and 43 that use of the minus $3 / 4$-in. sieve fraction data with Equation 3 appears to be a more reliable approach for these materials as compared with use of the minus No. 4 fraction. This may or may not be true for other materials. However, this is a logical finding in that the percent oversize is less for the minus $3 / 4-i n$. fraction and the fraction itself contains gravel. It is recalled that Torrey and Donaghe (1991) found that their large-scale compaction test procedure would satisfactorily replicate compaction parameters for a minus 3/4-in. material from the 6 -in. to the 12-in. to the 18-in. diam molds but would not satisfactorily do so for a minus No. 4 gradation. The presence of gravel in the minus 3/4-in. fraction makes it a closer relative of the total material, while the minus No. 4 fraction is a more distant cousin. However, it remains discouraging to see from Figures 42 and 43 that truly satisfactory predictions of maximum dry density of the total material from that of a fraction occur only for "windows" in gravel contents. At gravel contents both below and above that window, the predicted values may become excessively deviant from the actual values. One can live with some degree of overprediction of maximum dry density which occurs with increasing gravel content because it leads to conservative values of fill percent compaction. But, significant under-prediction of maximum dry density at lower gravel contents is a serious deficiency. Such serious under-prediction is evident in Figures 42 and 43 for gradations containing much less than 35 percent gravel. So, the current guidance in EM 1110-2-1911, Appendix B, that Equation 3 can be generally used for total materials containing up to 35 percent gravel is subject to question. However, it is far more likely that the guidance will prove valid if the prediction of maximum dry density is based on the minus $3 / 4-i n$. fraction than upon the minus No. 4 fraction.

61. Torrey and Donaghe's results relative to predictions of optimum water content using Equation 4 and the air-dry water content ( 0.6 percent) of the gravel are shown in Figures 44 and 45 . The trends seen in these two figures are also similar to that previously shown in Figure 40 for Donaghe and Townsend's (1975) data. A comparison of Figures 44 and 45 indicates that entering values of optimum water content of the minus $3 / 4-i n$. fraction into Equation (4) generally produces better predictions of optimum water content of 
the total material than does use of the minus No. 4 data for the same reasons as stated previously for predictions of maximum dry density. It is also seen from Figures 44 and 45 that plasticity of the minus No. 200 sieve fraction has a much greater impact on predictions of optimum water content based on the minus No. 4 fraction than on those based on the minus $3 / 4-i n$. sieve fraction. When gravel is added to the picture as it is for the minus 3/4-in. fraction, the influence of the plasticity of the minus No. 200 sieve material is essentially eliminated except for very low gravel contents, say of less than 10 percent. It is also seen from Figure 44, for predictions based on the minus No. 4 fraction, that the higher the plasticity of that fraction, the poorer the prediction yielded at lower gravel contents. Of course, the plasticity of the minus No. 4 fraction is dictated by the plasticity and percentage by weight of minus No. 200 sieve material. For the materials which Torrey and Donaghe (1991) tested, the percentage minus No. 200 ranged from 21 to 31 percent. The estimated bandwidth for silt (ML) fines shown in Figure 44 should be an approximate lower bound since those fines were nonplastic.

62. As previously pointed out, USACE (1977), Appendix B takes the water content of the oversized fraction used in Equations 2 and 4 to be the absorption, A, of the gravel. The absorption of a gravel is defined as its water content in a saturated surface-dry condition (ASTM (1990d)). While USACE (1970) does not mention the parameter, Appendix IV describes the saturated surface-dry condition and the value of the absorption can be calculated from the dry weight and saturated surface-dry weight obtained in the determination of bulk specific gravity $G_{m}$ or can be calculated from the apparent specific gravity $G_{a}$ and the bulk gravity by the equation:

$$
\text { Absorption, } A=\frac{G_{\mathrm{a}}-G_{\mathrm{m}}}{G_{\mathrm{a}} G_{\mathrm{m}}} \times 100 \text { percent }
$$

Use of the absorption in the equations is another avoidance of testing the total material. The alternative is to determine the water content of the gravel in the mix over a range in water content of the total material and for a range in gravel content. This would require working with large samples and large oven capacities. There is no obvious reason to presume that the oversized material exists in the mixture in a saturated-surface-dry condition. 
Its water content could conceivably be either less than or more than the saturated surface-dry value. It may or may not be a valid argument that the error would not be significant because whatever the actual water content of the fraction, it would not be very different from the absorption and, in Equations 2 and 4 , the value is multiplied by the percentage of coarse fraction which itself is usually less than 50 percent. The significance of any error introduced by use of the absorption must be judged against the specified range in water content and against the ordinary precisions of determining water content and optimum water content. Unfortunately, the authors are not aware of any investigations of water content of coarse aggregate in earth-rock mixtures at either natural or manipulated (wetted/dried) water content.

other correction methods

62. AASHTO AND USBR. Both the American Association of State Highway and Transportation officials (AASHTO) and the USBR employ an identical modified version of Equation 1 to predict maximum dry density of the total material. Both agencies use Equation 4 for predicting optimum water content. The AASHTO approach to predicting maximum dry density is contained within their test designation T224-86, "Standard Method for Correction for Coarse Particles in the Soil Compaction Test" and the USBR method is addressed in their test designation USBR 5515-89, "Procedure for Performing Laboratory Compaction of Soils Containing Gravel. The modified form of Equation 1 is as follows :

$$
\gamma_{\mathrm{tmax}}=\frac{R_{\mathrm{c}} \gamma_{\mathrm{fmax}} \gamma_{\mathrm{w}} G_{\mathrm{m}}}{f \gamma_{\mathrm{w}} G_{\mathrm{m}}+R_{\mathrm{c}} C \gamma_{\mathrm{fmax}}}
$$

where

$$
\begin{aligned}
\gamma_{\operatorname{tmax}}= & \text { calculated maximum dry density of the total material } \\
f_{\max }= & \text { maximum dry density of the finer fraction } \\
\gamma_{\mathrm{w}}= & \text { unit weight of water } \\
\gamma_{\mathrm{m}}= & \text { bulk specific gravity of the gravel } \\
\mathrm{c}= & \text { decimal percentage by weight of coarser fraction } \\
\mathrm{f}= & \text { decimal percentage by weight of finer fraction } \\
\mathrm{R}_{\mathrm{c}}= & \text { Fraction Density Factor defined as the decimal value } \\
& \text { of percent compaction of the finer fraction in the total } \\
& \text { material when the total material is at its maximum dry }
\end{aligned}
$$


density. The dry density of the finer fraction $\gamma_{f}$ is calculated from Equation 1 by entering the maximum dry density of the total material. Therefore, the Fraction Density Factor is equal to $\gamma_{f} / \gamma_{f \max }$ where $\gamma_{f \max }$ is determined in the 4 - or 6 -in. diameter mold

The modification of Equation 1 then amounts to substitution of the identity $\mathrm{R}_{\mathrm{c}} \gamma_{\mathrm{fmax}}$ for $\gamma_{f}$. This modification does not violate the basic weight-volume derivation of the equation and is in recognition of the fact that the presence of gravel affects the compaction of the fraction such that it may exist in the compacted mixture at some density other than its maximum dry density when the total material is at its maximum dry density. The factor $R_{c}$ is termed the Fraction Density Factor by the authors and is nothing more than the percent compaction of the fraction (expressed as a decimal) when the total material is at its maximum dry density. The authors added the subscript " $c$ " to the symbol for Fraction Density Factor to indicate that its value varies as the percent oversize c varies. The difference in the two organizations' practices lies in the difference in values of the Fraction Density Factor $R_{c}$ assigned to the given total material based on its gravel content. The authors have been unable to find a reference which describes how the AASHTO developed its values. The USBR has considerable testing experience with earth-rock mixtures. The USBR in its test designation 5155-89 provides three generic curves of percent compaction of the fraction, i.e., $R_{c}$ value or Fraction Density Factor versus gravel content for clayey gravel, silty gravel, and sandy gravel as shown in Figure 46. Figure 46 also shows the range in Fraction Density Factors values recommended by AASHTO. The USBR recommends use of their generic curves only in the absence of more "precise" data. However, there is little doubt that the generic curves have found the widest usage because more "precise" data for a specific material require the conduct of an extensive comparative testing program involving large-scale tests. The USBR uses its Rapid Compaction Method which is a short-cut method for obtaining the maximum dry density and optimum water content of the minus No. 4 fraction in conjunction with Equations 4 and 5 . This approach has been very popular in the private sector.

63. NAVFAC. Naval Facilities Engineering Command (NAVFAC) presents a different approach to correcting the value of maximum dry density of either the minus No. 4 fraction or the minus $3 / 4-i n$. fraction to obtain that of the total material. NAVFAC also uses Equation 4 for calculating optimum water 
content. The equation presented in US Department of the Navy (1982) is as follows:

$$
\gamma_{\mathrm{tmax}}=\frac{1-(0.05) F}{\frac{F}{162}+\frac{(1-F)}{\gamma_{\mathrm{fmax}}}}
$$

where

$$
\begin{aligned}
\gamma_{\mathrm{tmax}}= & \text { calculated maximum dry density of the total material, pcf } \\
\gamma_{\mathrm{fmax}}= & \text { laboratory maximum dry density of either the minus No. } 4 \\
& \text { or minus } 3 / 4-i n \text {. fraction, pcf } \\
F= & \text { percent coarser or oversize fraction expressed as a decimal }
\end{aligned}
$$

The constant value of 162 in Equation 6 represents the term $G_{m} \gamma_{w}$ so that a constant value of bulk specific gravity of the oversized particles of 2.59 is assumed. Of course, the appropriate value can be used. Recognizing that the term $(1-F)$ is the percent fine fraction expressed as a decimal, Equation 6 can be rearranged to the following form:

$$
\gamma_{t \max }=\frac{(1-0.05 c) \gamma_{t \max } 162}{162 f+C \gamma_{f \max }}
$$

where all terms are the same as in Equation 6 except that as in Equation 3:

$c=$ percent coarser (oversize) fraction expressed as a decimal

$\mathbf{f}=$ percent finer fraction expressed as a decimal

Equation 7 is readily seen to be Equation la multiplied by the factor $(1-.05 c)$ and taking a constant value of bulk specific gravity of the gravel as 2.59 . The NAVFAC DM 7.2 describes Equation 7 as a modified version of McLeod (1958). Examination of that ASTM reference reveals that McLeod's equation is precisely identical to Ziegler's. The authors have been unsuccessful at determining the Navy's rationale in applying the factor $(1-.05 \mathrm{c})$.

64. Equation 7 can be equated to Equation 5 to derive an equivalent Fraction Density Factor, $R_{c}$, for the NAVFAC method. That derivation yields: 


$$
R_{c}=\frac{162 f(1-.05 c)}{162 f+.05 c^{2} \gamma_{f \max }}
$$

The resulting equivalent Fraction Density Factors versus gravel content in the total material are also plotted in Figure 46.

65. Figures 47 and 48 compare Fraction Density Factors developed by Torrey and Donaghe (1991) for minus 3-in. and minus 2-in. gradations with the USBR, AASHTO, and derived NAVFAC values (Equation 8). Figure 47 shows WES factors based on taking the fine fraction as the minus No. 4 material while Figure 48 shows the factors based on the minus $3 / 4$-in. fraction. Taking the minus No. 4 material as the fine fraction, Figure 47 shows that Fraction Density Factors developed by Torrey and Donaghe (1991) for the minus No. 4 fraction were generally higher than those cited by the USBR and AASHTO although roughly parallel to those trends which are also roughly parallel to each other. The NAVFAC derived factors do not reflect nearly the sensitivity to increasing gravel content as compared with WES, USBR, and AASHTO but still fall within the range of the other three agencies' combined data. Taking the minus 3/4-in. material as the fine fraction, it is seen from Figure 48 that the WES factors are generally higher than the NAVFAC values. The USBR and AASHTO factors are not presumed to be applicable to the minus 3/4-in fraction and were not plotted in Figure 48. This is not to say that they have not been applied to the minus $3 / 4-i n$. fraction since they probably have. Obviously, neither the USBR, the AASHTO, nor the NAVFAC methods are satisfactory for the majority of the gradations tested by WES.

66. To add a more general dimension to the assessment of "generic" Fraction Density Factors, Figure 49 shows the factors calculated for tests performed by several different investigators on gradations containing clay fines. The reader is referred to the previous report in this series (Torrey and Donaghe 1991) for a discussion of the literature which includes the studies by the USBR (1963b), Donaghe and Townsend (1973), Donaghe and Townsend (1975), and Garga and Madureira (1985). The data of Figure 49 encompass maximum particle sizes from $3 / 4 \mathrm{in}$. to $4 \mathrm{in.,}$ a wide range in shape of grain-size distribution curves, percent minus No. 200 sieve approaching 40 percent, and plasticity of the fines (minus No. 200 sieve sizes) ranging from clay (CL) to clay $(\mathrm{CH})$. The authors believe that Figure 49 clearly indicates that the Fraction Density Factor for the minus No. 4 fraction is very much a function 
of the gradation and the plasticity and is not amenable to description by a single curve such as that of the USBR or NAVFAC or a narrow bandwidth such as that of AASHTO. Certainly, no consistent trends can be seen in Figure 49 in relative values of $R_{c}$ by maximum particle size since the factors for the minus 4-in. gradations plotted close to those for minus 3/4-in. gradations. However, it is seen from Figure 49 that the factors recommended by all three of those agencies fall within the bandwidth of the data from among the cited investigators. This indicates that all three agencies based their recommended factors on the data available to them which did not reflect a wide range in materials. This is consistent with the fact previously pointed out that relatively very few comparative testing programs involving large scale compaction testing have ever been conducted. The bandwidth in factors evident in Figure 49 is about 0.10 throughout the range in gravel content. The point to be made from the comparisons of Fraction Density Factors of Figure 491 ies in Figure 50 which shows the serious impact of very small variations in the factor on the calculated value of maximum dry density of the total material. It appears that it is not a wise practice to utilize Fraction Density Factors unless they have been established specifically for the materials at hand over their range in gradations and plasticities. The use of generic factors such as those offered by USBR, AASHTO, and NAVFAC may lead to major errors and failure to actually control compaction.

67. It is noteworthy from Figure 49 that for some gravelly materials the minus No. 4 fraction may exist in the compacted total material at a density higher than its maximum dry density when the total material is at its maximum value. This condition corresponds to a Fraction Density Factor greater than 1.00 . It must be remembered that the data of Figure 49 reflect compaction of the minus No. 4 fraction in either the 4 or 6 -in. diameter mold while all of the materials containing plus $3 / 4-i n$. sizes were compacted in larger molds. Torrey and Donaghe (1991) also compacted the minus No. 4 and minus 3/4-in. fractions in the same sized molds and using the same procedures as were used for the corresponding total materials. This practice provided data which were "corrected" for any equipment size and procedural effects. Figure 51 shows that Torrey and Donaghe developed Fraction Density Factors for minus No. 4 fractions "corrected" for equipment size and procedural effects which generally happened not to exceed a value of 1.00 . However, Figure 52 reveals that Fraction Density Factors for the minus 3/4-in. fractions which 
are also corrected for equipment size and procedural effects still exceeded a value of 1.00 for some gradations even up to 40 percent plus $3 / 4$-in. oversize. So, it can't be concluded that corrected Fraction Density Factors based on the minus No. 4 fraction for other gradations may not also exceed 1.00 . It must be remembered that in a compaction control procedure in the field, fractions would be compacted in the smaller molds so that the "uncorrected" Fraction density Factors are the applicable ones. In more general terms, Torrey and Donaghe (1991) found that it was not uncommon that both the minus No. 4 and minus $3 / 4-i n$. sieve fractions were brought to densities which were in excess of those for standard effort for their water contents within the total materials. In other words, those densities of the fractions were in excess of their individual standard effort compaction curves. In some cases for the corrected minus 3/4-in. fraction data as seen in Figure 52, the excess density also happened to exceed the maximum dry density for that fraction. It is beyond the scope of this report to enter into a discussion of how a fraction within a total material can be brought to a dry density in excess of its standard effort density for its water content. The reader is referred to the companion report of Torrey and Donaghe (1991) for that discussion.

\section{Basing the Specifications on the Compacted State of a Fraction of the Total Material}

Compacted state of a fraction

versus that of the total material

68. It was promised earlier that this report would discuss the practice of basing the compaction specifications on the engineering properties of a fraction of the total material upon the assumption that the compacted state of the total material is adequate if that of the fraction is adequate. It was previously pointed out that this approach has occasionally been used and generally without any testing of the total materials. The procedure in this case has been to establish the placement range in water content and a desired percent compaction based on laboratory shear testing of compacted specimens of either the minus No. 4 or minus $3 / 4-i n$. fractions representing the range of gradations encountered in the borrow sources. The minus No. 4 fraction has probably been chosen most often because it involves the least amount of material for both design testing and field compaction control procedures. The most popular USACE fill compaction control procedure employing a fraction has 
been the one- or two-point method. The family or families of compaction curves required to apply the one- or two-point method would be developed during the project design phase on the appropriate fractions over the range in gradations of the borrow materials. The USBR Rapid Compaction Control Method has been utilized in a few cases where the control was based on the minus No. 4 fraction. The remaining field procedures described below are judged to have been the most common. Sufficient material in addition to that excavated during the fill density test is taken as a grab sample from the location of the fill density test to perform the one- or two-point method. That material is scalped to obtain either the minus No. 4 or minus $3 / 4-i n$. fraction and to determine the percent oversize. The one- or two-point compaction control method will then yield a maximum dry density and optimum water content for the fraction.

69. The fill dry density of the fraction is calculated using Equation 1 from the fill dry density of the total material, the percent oversize of the fill sample and the bulk specific gravity for the gravel. However, in order to obtain a value of the fill dry density of the total material to enter into Equation 1, the fill water content of the total material must be known. The sand cone has been by far the most popular fill density test method and yields the wet density of the total material. In a very few cases, the fill water content of a sufficient size specimen of the total material derived from the grab sample has been determined by oven drying. However, oven drying of a specimen of the total material requires a large grab sample and large-capacity ovens. Therefore, the following round-about way of obtaining the fill water content of the total material using Equation 2 has been employed more often. To obtain the fill water content of the total material, the fill water content of a specimen of the fraction is first determined. If the selected fraction is the minus No. 4 fraction, an adequate sized water content specimen can usually be obtained from the material excavated as part of the fill density test. Having the fill water content of the fraction, Equation 2 is used with the absorption, A, of the gravel and the percent oversize of the fill density sample to calculate a value of fill water content of the total material. Given values of optimum water content and maximum dry density for the fraction obtained by the one- or two-point method, the fill water content of the fraction is compared with the specified range for that fraction and the fill dry density of the fraction used to calculate the percent compaction of the 
fraction to compare against its desired percent compaction.

70. Using the standard effort data obtained by Torrey and Donaghe (1991), it is possible to calculate the percent compaction of the total materials given the densities of the minus $3 / 4 \mathrm{in}$. and minus No. 4 fractions corresponding to some typical desired percent compaction for the fraction, say 95 percent. The minus $3-i n$., minus $2-i n$. and minus 3/4-in. gradations shown in Figures 24 and 25 and described in Table 15 were treated as total materials when calculations were based on the minus No. 4 fraction. Calculations based on the minus 3/4-in. fraction treated the minus 3 -in. and minus 2-in. gradations. Torrey and Donaghe obtained values of maximum dry density for each of these gradations and their minus 3/4-in. and minus No. 4 fractions. For each gradation, the dry densities of the minus $3 / 4-i n$, and minus No. 4 fractions equivalent to 95 percent of their maximum dry densities were entered into Equation 3 to calculate the corresponding dry densities of the total material. The calculated dry density of each total material was then compared with its maximum dry density to calculate the percent compaction corresponding to the two fractions at 95 percent compaction. Curves fit to the results of these calculations for both silt and clay fines (minus No. 200 sieve) are shown in Figure 53. First of all, it is seen from Figure 53 that the two sets of data for the two fractions for each type of fines were not distinguishable from each other with respect to trends. Therefore, one curve represents both fractions for each type of fines except that the maximum percent oversize for the minus 3/4-in. fractions was 36 percent. When the fractions are at 95 percent compaction, the percent compaction of the total material is less than 95 percent up to about a gravel content of 30 percent for the case of silt fines and up to about 35 percent for clay fines. The maximum deviation of the percent compaction of the total material below that of the fractions is only about one percentage point for the gradations containing silt fines and about two percentage points for the case of clay fines. Overall, there is surprisingly little difference between the results obtained by Torrey and Donaghe for the gradations containing clay fines and those containing the nonplastic silt fines.

71. Figure 54 is of the same nature as Figure 53 but treats all the data pertaining to clay fines and the minus No. 4 fraction reported by the previously referenced investigators. The lower bounding curve indicated in Figure 54 happens to be that obtained by Torrey and Donaghe for clay fines of 
Figure 53. The upper bounding curve is derived from the standard effort data of Donaghe and Townsend (1975) for minus 3/4-in. (scalped/ replaced) gradations. It is recalled that the Fraction Density Factor $R_{c}$ is the percent compaction of the fraction when the total material is at its maximum dry density. Figure 49 showed the range in Fraction Density Factors based on the minus No. 4 fraction obtained by the several investigators for a wide range in gradations and maximum particle sizes of earth-rock mixtures containing clay fines. As would be anticipated on the basis of Figure 54, the upper and lower bounding values of Fraction Density Factor in Figure 49 also correspond to the data of Torrey and Donaghe (1991) and Donaghe and Townsend (1975), respective$1 y$, but the order, i.e., upper versus lower, is reversed compared with Figure 54. If a lower percent compaction of the fraction is required to produce 100 percent compaction of the total material (lower Fraction Density Factor), a fixed percent compaction of the fraction, i.e., 95 percent, will result in materials with lower Fraction Density Factors exhibiting higher percent compaction of the total material. However, it is recalled that the authors stated previously that there were no trends seen in Fraction Density Factors discernable from Figure 49 which could be easily tied to gradation or plasticity of fines. Therefore, given the gradation and plasticity of some total material, there is no way to say where within the bandwidth of Figure 54 it may fall.

72. Figure 54 implies that most total materials at their optimum water contents will exhibit a percent compaction equal to or greater than that of the minus No. 4 fraction. However, Figure 54 also shows that, for some gradations, the total material would have to be brought to densities in excess of 100 percent compaction in order to achieve 95 percent compaction of the minus No. 4 fraction. For the minus 3/4-in. data of Donaghe and Townsend (1975), this is seen to occur at a gravel content of only about 25 percent. This would seem to pose a potential problem in achieving such densities of the total materials. However, at 95 percent of standard effort maximum dry density an earth-rock material is in a relatively loose state. Higher degree of compaction is often easily achieved by typical compaction equipment and common combinations of loose lift thickness and number of passes.

73. Figure 55 presents trends in percent compaction letween the total materials and their minus No. 4 fractions in a converse manrer to Figure 54. In Figure 42, the variation of the percent compaction of the fraction is shown 
for fixed percent compactions of the total material. One purpose of this Figure is to indicate how the trend in percent compaction of the fraction shifts as the percent compaction of the total material changes. The compaction data used were those reported for tlay fines by Torrey and Donaghe (1991). Of course, the curve of Figure 55 corresponding to 100 percent compaction of the total material represents the trend in Fraction Density Factor with gravel content. This is the curve of best fit to the data of Torrey and Donaghe plotted in the format of Figure 49. The curve shown in Figure 55 corresponding to the total material at 95 percent compaction shows that it is approximately parallel to the curve for Fraction Density Factor for gravel contents above its peak value which is around 15 percent. There is another important observation from Figure 55 which has already been addressed in previous discussions of Ziegler's Equation $1 \mathrm{a}$ and its modified form Equation 3 . Note from Figure 55 that when the total material is at its maximum dry density (100 percent compaction), the minus No. 4 fraction is also at its maximum dry density for one and only one gravel content. Likewise, when the total material is at 95 percent compaction, there is only one gravel content at which the fraction is also at 95 percent compaction. The same would be true for the minus 3/4-in. fraction since those data were not separable in trend in Figure 53 from the data based on the minus No. 4 fraction. This means that if the maximum dry density of the fraction is entered into Equation 3, the calculated value of dry density for the total material will not be its maximum dry density except for a single value of gravel content. This was the reason previously pointed out that other agencies have modified Equation 3 by inclusion of a Fraction Density Factor to obtain Equation 5.

74. In addition to the trends in density as discussed above, the relative trends in water content between a total material and a fraction can also be examined. Figure 56 indicates how the water content of the total materials varied with respect to their optimum values when the minus $3 / 4-i n$. and minus No. 4 fractions were at their optimum water contents for the gradations tested by Torrey and Donaghe (1991). The water content of each total material was calculated using Equation $2 \mathrm{a}$ by entering the optimum water content of the minus $3 / 4-i n$. and minus No. 4 fraction and the corresponding percent finer fraction $f$ and percent oversize c. The data based on the minus 3/4-in. fraction could not be separated in trend by type of fines (minus No. 200 sieve sizes) while those based on the minus No. 4 fraction showed a clear influence 
of character of fines. Figure 57 is in the same format as Figure 56 and shows the range in relative water content of the total materials containing clay fines for the minus No. 4 fraction at optimum water content pertaining to the data of previously cited investigators. Again, the upper bound curve represents the data of Torrey and Donaghe (1991) and the lower bound curve represents the data for minus 3/4-in. gradations tested by Donaghe and Townsend (1975). Like the variation of percent compaction of the total material in Figure 54, the variation of water content of the total material with respect to its optimum when the fraction is at its optimum is also a complex function of gradation and plasticity of fines (minus No. 200 sieve sizes). As was stated for percent compaction of the total material shown in Figure 54, knowledge of gradation and plasticity of fines does not permit a placement of a given total material in a relative position within the bandwidth of Figure 57. 75. Figure 58 shows how the water content of the total material varies with respect to its optimum when the minus No. 4 fraction water content is varied in fixed increments with respect to its optimum. Torrey and Donaghe's (1991) data for clay fines are used and the curve of Figure 58 for the fraction at its optimum is the same as the dashed symbol curve of Figure 56 . Figure 58 shows that change in water content of the total material is generally less than the change in water content of the fraction. The three curves are not parallel. With increasing gravel content, the change in relative water content of the total material becomes increasingly less sensitive to changes in water content of the fraction. This is logical since the physical quantity of minus No. 4 material is steadily decreasing. At about 22 percent gravel the change in relative water content of the total material is about threefourths of the change in relative water content of the minus No. 4 fraction (minus and plus one percentage point from optimum) while at 60 percent gravel it is only about one-third.

Shear strength of a fraction versus that of the total material

76. The previous discussion of density of a fraction versus density of the total material was based on a minimum desired percent compaction for a fraction of 95 percent of standard effort maximum dry density. This is a very common standard for USACE Civil Works embankment construction. Of course, that desired degree of compaction is not simply adopted as a stancard practice, but is established along with the specified range in placement water 
content for each project as the result of design considerations utilizing considerable laboratory testing of the various soils. One of the most important of those design considerations is the shear strength of the soil which reflects, among other things, its state of compaction. Although it is not the principal subject of this report, the authors wish to spend a few words on the subject of the comparative shear strengths of the total material versus the minus No. 4 fraction at densities corresponding to 95 percent of their standard effort maximun dry densities. The general presumption has existed that use of shear strengths of a fraction is a conservative practice, i.e., that the strength of the total materials brought to the same percent compaction would be considerably greater. The following will provide some assessment of that presumption.

77. Donaghe and Torrey (1985) performed a comparative shear strength testing program employing 15-in. diameter by 37-in. tall triaxial specimens of minus 3-in. total materials and 6 -in. diameter by 13.6-in. tall triaxial specimens for the minus No. 4 fractions. As shown in Figure 59, they tested several artificially blended gradations and one gradation typical of one major material placed in the embankment of DeGray Dam, Caddo River, Arkansas. Unconsolidated undrained ( $Q$ test) and consolidated undrained ( $R$ test with pore pressure measurements) were performed on specimens of the blended materials and their minus No. 4 fractions. Only an $R$ test series was performed on the DeGray Dam soil. All test specimens were compacted to initial densities targeted at 95 percent of standard effort maximum dry density. In strength testing for design of compacted clayey fills, experience has resulted in a customary practice of beginning the process of establishing compaction specifications by first testing specimens compacted to 95 percent of standard effort maximum dry density at optimum water content plus two percentage points. The wet side water content is chosen simply because for a constant dry density, shear strength declines with increasing water content. If the shear strengths, other engineering properties and other considerations pertaining to construction are more than adequate at optimum plus two percentage points, testing of wetter specimens may eventually be included. of course, testing of specimens to the dry side of optimum is also included to complete the consideration of all pertinent properties. Donaghe and Torrey (1985) prepared their test specimens to be in the vicinity of optimum water content plus two percentage points by adding saturated surface-dry gravel to the minus 
No. 4 material prepared to a water content one percentage point wet of its optimum.

78. Compaction characteristics of soils containing more than 10 percent minus No. 200 sieve sizes are determined by the impact compaction test which yields the typical parabaloid moisture-density curve exhibiting a maximum dry density at an optimum water content. Soils containing less than five percent minus No. 200 sieve sizes are typically termed clean, cohesionless soils and compaction parameters are based on laboratory tests to determine maximum and minimum densities. Fill compaction control of these clean, cohesionless soils is usually based on relative density, i.e., the relative position of the value of fill density between the values of maximum and minimum density expressed as a percent. Soils which contain five to 10 percent minus No. 200 sieve sizes represent a gray area where a decision has to be made as to which way to treat compaction control, i.e., by impact compaction or relative density, depending on the plasticity of the fines. If the fines are plastic clay, the impact compaction curve applies. If the fines are silt, the decision may go either way since such materials may yield a typical impact compaction curve and also exhibit easily obtained and consistent laboratory values of maximum and minimum densities.

79. For minus No. 4 sieve materials (and smaller maximum particle size soils) amenable to impact compaction testing, optimum water content typically corresponds to a degree of saturation between 50 and 90 percent. As gravel is added to any given minus No. 4 material, the degree of saturation at optimum water content will either increase or decrease by only a small amount as a result of shifts in position of the compaction curve and because of change in specific gravity of the mixture. Therefore, Q test specimens (unconsolidated undrained) of a compacted earth-rock mixture prepared at 95 percent compaction and near optimum water content are probably at degrees of saturation somewhat less than 90 percent before application of the confining pressure and shearing. Q tests on partially saturated specimens typically yield both a total stress angle of internal friction, $\phi$, and some cohesion intercept, $c$. In reality, it is a misnomer to refer to a $Q$ strength envelope for a partially saturated soil because the test specimen consolidates (densifies) under the applied normal stress (confining pressure) before shearing as a result of compression of the air in the voids. Furthermore, the shearing of the specimen does not occur under undrained conditions because again the specimen can 
change volume with compression or decompression of the pore air. So, in the end, a $Q$ test series performed on a partially saturated soil is really an undefined mixed version of the consolidated undrained (R) test and the consolidated drained (S) test. The $\phi$ angle seen from the partially saturated $Q$ test series reflects the increased densification of each test specimen with increase in normal stress (confining pressure) and some freedom to change volume during shear. At higher confining pressures it is possible that the initially partially saturated test specimen will consolidate sufficiently such that the degree of saturation is increased into the upper 90 percentile before shear. At degrees of saturation above about 95 percent, the specimen will behave essentially as if saturated and the $Q$ strength will theoretically become a point on the consolidated undrained ( $R$ ) envelope. However, saturated $Q$ test results cannot be ordinarily be matched to a point on the $R$ strength envelope because the effective stresses are not known for the $Q$ test and both the $Q$ and $R$ test reflect their own types of sample disturbance effects, While the so-called $Q$ shear strengths of partially saturated soils do not conform to the classic concept of the unconsolidated undrained shear strength, they may remain appropriate for assessing the "after construction " stability of a moderately high new embankment because the fill will likely be partially saturated at the end of construction except, perhaps, for lower portions which may have been consolidated to near saturation under the weight of overlying material.

80. Donaghe and Torrey (1985) obtained $Q$ test data for blended material specimens prepared to 95 percent of their standard effort maximum dry densities. Water contents of the minus No. 4 fraction specimens were one percentage point wet of optimum while those of the total materials resulted from adding saturated surface-dry gravel to the fraction at one percentage point on the wet side. Thus, the total material containing 20 percent gravel was tested at 1.4 percentage points wet of optimum, that containing 40 percent gravel at 1.5 percentage points wet, and that containing 60 percent gravel at 0.1 percentage points wet. Two confining pressures of 4.32 tsf and 14.40 tsf were utilized. Figure 60 presents the results of those tests. It is seen form Figure 60 that the $Q$ strength envelopes of the total materials and their corresponding minus No. 4 fractions are not identical and that the difference in $\phi$ angles tends to increase with increasing gravel content in the total material. At the lowest gravel content of 20 percent the two envelopes are 
reasonably close together. At lower confining pressures the $Q$ strength of the fraction exceeds that of the total material and is less than that of the total material at higher confining pressure. As a reference point, 10 tsf of confining pressure is equivalent to about $140 \mathrm{ft}$ of $\mathrm{fill}$ at maximum wet density using average numbers for the blended material. Figure 60 indicates that for gradation No. 2 with 40 percent gravel, the $Q$ strength of the fraction is greater than that of the total material below a confining pressure of about 4 tsf. That confining pressure is equivalent to about $55 \mathrm{ft}$ of $\mathrm{fill}$. Therefore, if the $Q$ strength of the fraction were used to assess after construction stability, it would be an unconservative strength for any point within the fill which is less than about $55 \mathrm{ft}$ from the surface of the embankment. On the other hand, for depths corresponding to $10 \mathrm{tsf}$ or higher, use of the $Q$ strength of the fraction for the gradations containing 40 and 60 percent gravel would certainly be very conservative.

81. Figures 61 and 62 present the $R$ strength envelopes obtained by Donaghe and Torrey (1985) for the blended and DeGray Dam materials, respectively. For the blended materials the R strengths of the minus No. 4 fractions were less than those for the corresponding total materials. For the gradation containing 60 percent gravel there was very little difference in the $\mathrm{R}$ strengths between the total material and its fraction. However, for the gradations containing 20 and 40 percent gravel, use of the $R$ strength of the fraction would be a somewhat conservative practice. Where the single DeGray Dam gradation containing 48 percent gravel is concerned, there is no significant difference between the $R$ strength of the total material and its minus No. 4 fraction. So, where $R$ strengths are concerned, the strength of the fraction may or may not be representative of the total material, but any deviation would appear to most likely be toward the conservative side.

82. The most interesting aspect of the $R$ test data is seen from the effective stress paths shown in Figures 63 through 65 for the blended material and in Figure 66 for the DeGray material. For the blended materials, Figures 63 through 65 show that as gravel content increased, the test specimens exhibited increasing induce pore water pressures during shear. The DeGray material with 48 percent gravel (Figure 66) exhibited even more induced pore water pressure than did the blended material with 60 percent gravel (Figure 65 ). These tendencies for the materials to contract or reduce their volumes during shear reflects their relative compactness. All specimens were 
prepared to initial densities corresponding to 95 percent of standard effort maximum dry density. It is seen from Figures 61 and 62 that they consolidated to percent compactions from 96 to 100 percent under the applied confining pressures before undrained shear. Note from Figure 61 for the blended material that the higher confining pressure resulted in percent compaction of the gradations containing 40 and 60 percent gravel of about 100 percent. Nonetheless, these two gradations still yielded increasing tendency to contract during shear with increasing gravel content. Therefore, it is concluded that earth-rock mixtures compacted to 95 percent of maximum dry density are in a relatively loose state and may remain so with increasing gravel content even up to 100 percent compaction. The considerable pore water pressures generated during undrained shear account for the surprisingly low $\mathrm{R}$ strengths seen in Figures 61 and 62. This apparently relatively loose state at 95 percent compaction has been borne out in fill placement experience in that there have generally been no problems in attaining that degree of compaction for earthrock mixtures. The tendency to generate high pore pressures during undrained shear would perhaps be of greatest concern under earthquake loadings. In that case, lose of strength due to elevated pore pressure generated by shaking (cyclic loading), if sufficiently severe and sustained, might lead to serious deformations of the embankment. 
PART VI: A UNIFIED VIEW OF THE EFFECTS OF GRAVEL ON THE COMPACTION OF THE MINUS NO. 4 OR MINUS 3/4-IN. FRACTION

\section{Maximum Dry Density}

83. The USBR and AASHTO approach to predicting the maximum dry density of the total material employing a modified version of Equation 3 ,i.e, Equation 5 was previously discussed. Equation 5 includes the Fraction Density Factor, $R_{c}$, which is the decimal expression of the percent compaction of a fraction in the total material when the total material is at its maximum dry density based on the dry density calculated for the fraction from Equation 1. It was shown (see Figure 49) that the Fraction Density Factor versus gravel content relationships resulting from several investigations varied over such a wide range that use of single curves or a narrow band such as those recommended by the USBR and NAVFAC or AASHTO, respectively, (see Figure 46) could result in unacceptably inaccurate predictions of maximum dry density of the total material using Equation 5. It was also shown (see Figure 50) that the calculated value of maximum dry density of the total material is very sensitive to small differences in the Fraction Density Factor.

84. Equation 5 interrelates the percent compaction of the fraction, the maximum dry density of the total material, the percent coarser fraction (oversize), percent finer fraction and the bulk specific gravity of the gravel. The value of percent compaction of the minus No, 4 or minus $3 / 4-i n$. fraction (Fraction Density Factor) can be normalized by division by the percent gravel in the total material times the bulk specific gravity. The authors choose to call this parameter the "Density Interference Coefficient", $I_{c}$, which is defined as follows:

$$
I_{c}=\frac{R_{c}}{P_{g} G_{m}}
$$

where

$$
\begin{aligned}
& \mathrm{R}_{\mathrm{c}}=\text { decimal value of percent compaction of the fraction (Fraction } \\
& \text { Density Factor) } \\
& \mathrm{P}_{\mathrm{B}}=\text { decimal value of percent gravel in total material }
\end{aligned}
$$


$\mathrm{G}_{\mathrm{m}}=$ bulk specific gravity of the gravel

Note from Equation 9 that if the Density Interference Coefficient is based on the minus No. 4 fraction, $P_{g}$ is equivalent to the percent oversize $c$ and the coefficient approaches infinity as $\mathrm{P}_{8}$ approaches zero. If the Density Interference Coefficient is based on the minus $3 / 4-i n$. fraction, $P_{g}$ cannot be less than the gravel content in that fraction so that the coefficient will have a maximum value corresponding to that percent gravel.

85. Smooth curves of this parameter versus gravel content were obtained for the minus No. 4 fraction by Torrey and Donaghe (1991), as shown in Figure 67 for both silt and clay fines. Figure 68 shows similar results if the percent compaction of the minus $3 / 4-i n$. fraction is treated in the same manner. Note that when the Density Interference Coefficient is based on the minus No. 4 fraction, the percent gravel in the total material $P_{g}$ is equivalent to the percent oversize c. However, when the coefficient is based on the minus $3 / 4-i n$. fraction this is no longer true. Figure 69 confirms similar results for the minus No. 4 fraction data of Garga and Madureira (1985), Figure 70 for the minus No. 4 fraction data of Donaghe and Townsend (1975) and the USBR (1963) and Figure 71 for the results reported by Gordon, Hammond, and Miller (1964). All other things being identical, the authors reason that a difference only in bulk specific gravity of the gravel would shift a given value of maximum dry density but not the optimum water content. Therefore, the bulk specific gravity is utilized in the calculation of the Density Interference Coefficient because it appeared in the study of available data that its use might reduce the coefficient to a single curve for gravelly soils from one geological environment but exhibiting variable bulk gravities. This remains to be verified.

86. It is gratifying to realize from Figures 67 through 71 that a smooth curve can indeed be fitted to each data set even though whole families of gradation curves are represented, including not only variable gravel content but also variable percent fines and variable maximum particle size. The Torrey and Donaghe data of Figures 67 and 68 were derived from the gradation curves shown in Figures 24 and 25 and summarized in Table 15. The Garga and Madureira (1985) data of Figure 69 represents a range in maximum particle size, linear gravel gradations, and variable minus No. 4 fractions pertaining to Sao Simao Dam (Brazil) materials as indicated in Figure 22 and Table 14. The minus 3 -in. full-scale gradation and minus 3/4-in. scalped/replaced data 
of Donaghe and Townsend (1975) of Figure 70 also represent a significant range in gradations shown in Figures 20 and 21 and described in Table 13. The USBR (1963) data also shown in Figure 70 represent the minus $3-i n$. to minus 3/8-in. gradations of Figure 15. The Gordon, Hammond and Miller data of Figure 71 were derived from the six families of gradation curves of Oroville Dam materials seen in Figures 17 and 18 and described in Table 10.

87. There are several important observations to be made from Figures 67 through 71. When the Interference Coefficients obtained by Torrey and Donaghe of Figures 67 and 68 for the minus No. 4 and minus 3/4-in. fractions, respectively, are compared, it is seen by overlaying the two figures that the coefficients appear to describe one curve. Garga and Madureira's data of Figure 69 shows some separation of the trends by compactive effort which appears trivial. However, just as for the Fraction Density Factor, a back-calculation of maximum dry density of the total material is also sensitive to very small changes in the Density Interference Coefficient as will be shown in the next section of this report. Also, it is seen in Figure 70 for Donaghe and Townsend's (1975) data that the scalped and replaced gradations produced a different curve of Density Interference Coefficient versus gravel content in the total material as compared with that indicated for the minus 3 -in. parent gradations. This is additional evidence that scalping/replacing in effect generates a different genre of materials. Donaghe and Townsend (1975) also tested minus 3-in. full-scale and associated minus 3/4-in. scalped/replaced gradations containing 40 percent gravel and variable fines of 15,25 , and 35 percent fines (minus No: 200 sieve). Figure 70 shows the data from those tests to also fall on the respective Density Interference Coefficient curves for the major test program for which the percent fines was fixed at 25 percent (see Figures 20 and 21). So, it appears that for a range in gradations of gravelly soils as would generally be obtained from geologically similar project borrow sources that a single smooth curve of Density Interference Coefficient, $I_{c}$, versus gravel content in the total material can be developed for either the minus No. 4 or minus $3 / 4-i n$. fractions for a given compactive effort. Density Interference Coefficients developed by treating fractions of those gradations as full-scale materials will lie on the same curve as those for the parent gradations but coefficients developed for derivative scalped and replaced gradations will not. 
Predicting the maximum dry

density of the total material using

using Density Interference Coefficients

88. Figure 72 indicates the degree to which the value of maximum dry density of the total material back-calculated on the basis of the Density Interference Coefficient is sensitive to small variations in the factor.

Because of that sensitivity, it is necessary to assess the practicality of use of the curve for prediction purposes. In other words, if a smooth curve is fitted by eye to the Density Interference Coefficient versus gravel content data, will values picked off that curve result in a satisfactorily accurate prediction of the maximum dry density of the total material? The prediction procedure amounts to calculating the percent compaction of the fraction in the total material (Fraction Density Factor) from the Density Interference Coefficient from Equation 9 given the gravel content and the bulk specific gravity. The Fraction Density Factor can then be entered into Equation 5 along with the maximum dry density of the fraction to calculate the maximum dry density of the total material. Equation 5 can be restated in terms of the Density Interference Coefficient, $I_{c}$, by first rearranging Equation 9 as follows:

$$
R_{\mathrm{c}}=I_{\mathrm{c}} P_{\mathrm{B}} G_{\mathrm{m}}
$$

where

$$
\begin{aligned}
& \mathrm{R}_{\mathrm{c}}=\text { decimal value of percent compaction of the fraction (Fraction } \\
& \text { Density Factor) } \\
& \mathrm{P}_{\mathrm{g}}=\text { decimal value of percent gravel in total material } \\
& \mathrm{G}_{\mathrm{m}}=\text { bulk specific gravity of the gravel }
\end{aligned}
$$

substituting for $R_{c}$ from Equation 10 into Equation 5:

$$
\gamma_{t \max }=\frac{P_{g} I_{c} \gamma_{f \max } \gamma_{w} G_{m}}{f \gamma_{w}+P_{B} C I_{c} \gamma_{\max }}
$$

where

$$
\begin{aligned}
\gamma_{t \max } & =\text { predicted maximum dry density of the total material, pcf } \\
\gamma_{w} & =\text { unit weight of water or } 62.4 \text { pcf } \\
f & =\text { decimal value of percent finer fraction by weight }
\end{aligned}
$$


c - decimal value of percent oversize by weight which is equal $P_{B}$
if $I_{c}$ is based on the minus No. 4 fraction If the bulk specific gravity of the gravels associated with a project is not a variable, it need not be used in the calculation of the Density Interference Coefficient. Equations 9 and 11 above would be altered accordingly. The only effect would be a scaling upward of the numerical values of the coefficient and the absence of the bulk specific gravity in back-calculation of the maximum dry density of the total material using the coefficient. It is emphasized that there must be no presumption that the bulk gravities of the gravel portions of fractions of a total material are all the same since the breakdown of the parent geological materials into different sizes may reflect mineralogy. It would be wise to verify these numbers by testing each gravel fraction.

89. Figure 73 presents the results of prediction of the maximum dry density of the total material using Equation 11 with the Density Interference Coefficient based on the minus No. 4 fractions of the gradations tested by Torrey and Donaghe (1991). Figure 74 presents the prediction results using Torrey and Donaghe's Density Interference Coefficients based on the minus 3/4-in. fraction. In both these cases, a Density Interference Coefficient versus gravel content curve was drawn through the average values of Figures 67 and 68 taking the silt and clay fines data together as one set. To do this, it was found necessary to plot the data of Figures 67 and 68 to a sufficiently large scale to allow picking of values from the curve with a good estimate of the third decimal place in the value of Density Interference Coefficient. For sake of simplicity, the two percent precision limits shown in Figures 73 and 74 (and in similar figures to follow) were calculated by taking two percent of the actual value of maximum dry density of the total material. The rigorously correct way to apply the precision limit would be to average each pair of actual and predicted values and then take two percent of that number. However, the simplified approach does not result in any comparative data points falling within the approximate precision limits shown when in fact they are actually outside those limits by the correct calculation. Figures 73 and 74 both show that for the materials tested by Torrey and Donaghe that average Density Interference Coefficients based on either the minus No. 4 or minus 3/4-in. fractions will result in excellent predictions of maximum dry density of the total material for gradations containing either silt or clay fines. The authors emphasize that this finding only applies to the materials 
tested by Torrey and Donaghe. Other materials may differ significantly on the basis of plasticity of fines. Close comparison of Figures 73 and 74 show no significant differences between quality of predictions obtained from coefficients based on the minus No. 4 and minus $3 / 4-i n$. fractions. It was previously pointed out that the Interference Coefficients based on the two fractions obtained by Torrey and Donaghe appeared to lie on the same curve (compare Figures 67 and 68).

90. Figure 75 presents results of predictions of maximum dry density of the total materials using the data reported by Garga and Madureira (1985). In this case, separate Density Interference Coefficient (based on the minus No. 4 fractions) versus gravel content curves were used for each data set by compactive effort. This was required because the very small shifts in the data seen in Figure 48 with compactive effort were significant. Figure 75 shows that, in general, the predictions of maximum dry densities of the total materials fell within a two percent precision range of the actual values.

91. It would not be a significant exercise to predict maximum dry densities of the total materials using the data of Donaghe and Townsend (1975) or USBR (1963) because only one set of gradations were used in those studies. Therefore, a smooth Density Interference Coefficient versus gravel content curve could be almost precisely fitted through those respective data and almost exact replication of actual maximum dry densities of the total materials would result.

92. Figure 76 presents the results of predictions of maximum dry densities of the total materials using the data reported by Gordon, Hammond and Miller (1964). Again, the Density Interference Coefficient (based on the minus No. 4 fractions) versus gravel content curve was fitted by eye to the data of Figure 71. The prediction method for these data was complicated by the fact that gravel portions of fractions of those materials had different bulk specific gravities which ranged from 2.79 for the smaller sizes up to 2.85 for the larger sizes. Generic rather than exact gradations of the test specimens were provided so that only crude weighted values of bulk specific gravity could be calculated using the percentages by weight of each gravel size range in the generic gradations. Even though Figure 76 shows excellent results in prediction of maximum dry densities of the total materials, the authors believe the results would have been better if the bulk specific gravity had been available for each tested gradation's gravel fraction taken as a 
whole.

93. Because the Density Interference Coefficients calculated for several of the clayey gravels tested by the several investigators appeared to correspond, it was decided to lump those data together as in Figure 77 and again predict the maximum dry densities of the total materials. Figure 78 shows relatively good predictions of maximum dry densities of the total materials using the Density Interference Coefficient curve shown in Figure 77 . It is to be noted that the data of Gordon, Hammond, and Miller, which were also for a clayey gravel compacted at standard effort, are not included in Figures 77 and 78. It was found that their Density Interference Coefficients generally trended significantly lower than all the other investigators. This is an indicator that, just as for Fraction Density Factors, it should not be presumed that there is one generic Density Interference Coefficient curve for all clayey gravels. In fact, if the data of Figure 77 are examined closely, subtle differences in Density Interference Coefficients are indicated for each data set by their groupings.

Developing Density Interference Coefficients without large-scale compaction on the total material

94. In practice, very few agencies, consultants or contractors have the capability to perform compaction tests on total materials in large molds. It was previously demonstrated that Density Interference Coefficients determined on fractions of the total material treated as total materials in their own right fall on the same curve of Density Interference Coefficient versus gravel content as the parent total materials. The general shape of the Density Interference Coefficient versus gravel content curve suggests that it might plot as a straight line in $\log -\log$ coordinates. Figure 79 presents the data of Figure 77 replotted in this manner with the addition of the Gordon, Hammond, and Miller data of Figure 71. It is seen from Figure 79 that the data trends are linear for all investigators between 10 and about 45 percent gravel. Above about 45 percent gravel, the data trends are no longer linear in the log-log space but seem to become linear and parallel in cartesian coordinates as shown in Figure 80 . The apparent linearity between 10 and 45 percent gravel in $\log -\log$ coordinates offers the strong possibility that fractions of the total materials compacted in smaller molds may be used to establish the Density Interference Coefficient versus gravel content curve for gravel 
contents in the total materials up to 45 percent. Establishment of the Density Interference Coefficients using fractions and small molds could be achieved as follows:

a. Select representative total material gradations which span the range encountered in the borrow source. As a precaution, treat separate borrow sources separately.

b. Obtain representative samples of the minus 3/4-in. fractions and corresponding samples of the minus No. 4 fractions of those representative minus $3 / 4-i n$. fractions.

c. Determine the gravel contents and bulk specific gravities of the minus $3 / 4-i n$. fractions.

d. Perform compaction tests in the 6-in. diameter mold on the minus $3 / 4-i n$. fractions and on the minus No. 4 fractions to determine the maximum dry densities for each.

e. Treat each minus $3 / 4-i n$. fraction as if it were a total material. Use its gravel content, bulk specific gravity and maximum dry density with Equation 1 to compute the dry density of the corresponding minus No. 4 fraction. Calculate the percent compaction of the minus No. 4 fraction and then the respective Density Interference Coefficient by Equation 9 .

f. Plot, in $\log -\log$ coordinates, the Density Interference Coefficients versus the respective gravel contents of the minus $3 / 4-i n$. fractions and carefully fit a straight line through the data points from 10 percent up to 45 percent gravel. Do not presume the linear fit to be good below 10 percent gravel. The data below 10 percent gravel should not be linear and would have to be determined by testing minus $3 / 4-i n$. fractions with gravel contents less than 10 percent.

g. Convert and plot the $\log -\log$ straight line to cartesian coordinates at a scale permitting estimation of the Density Interference Coefficient to the third decimal place.

h. Accept the cartesian coordinate curve of Density Interference Coeffi-cient versus gravel content for predicting the maximum dry density of the total materials from the borrow source containing up to 45 percent gravel using Equation 10. If a minus $3 / 4-i n$. fraction happens to contain 50 percent or more gravel, it may be feasible to fit the linear higher gravel content portion of the curve through that data using a slope of 0.0132 (see Figure 80 ). This would require joining the two curve segments together with a smooth curve between 45 and 50 percent grave1.

95. It must be realized that the above procedure will not account for different values of maximum dry density of total materials which might be obtained for a single material from the variety of large-scale compaction equipment and procedures which has been employed. There is no widely accepted standard large-scale test. However, the authors are willing to venture the 
opinion that in the absence of a standard compaction test for soils containing large particles, that the values predicted by the above procedure would be as "good" as any obtained from some large-scale compaction test. Obviously, should the capability to perform large scale tests be available, the Density Interference Coefficients should be calculated using maximum dry densities of the total materials obtained in the appropriate large mold. Should large scale equipment not be available, the authors suggest that the minus 1 -in. fraction may be used with the 6 -in. mold in the short-cut procedure described above in order to gain the maximum range in gravel content in the fraction. However, the current edition of USACE (1970) does not prescribe this practice.

\section{Optimum Water Content}

96. In a manner somewhat analogous to the Density Interference Coefficient $I_{c}$ the optimum water contents of fractions and corresponding total materials can be used to calculate a simple factor which tracks the influence of gravel content as follows:

$$
F_{\text {opt }}=\frac{\frac{W_{\text {fopt }}}{W_{\text {topt }}}}{P_{g}}
$$

where

$$
\begin{aligned}
\mathrm{F}_{\text {opt }} & =\text { optimum Water Content Factor } \\
\mathrm{W}_{\text {fopt }} & =\text { optimum water content of the finer fraction, percent } \\
\mathrm{W}_{\text {topt }} & =\text { optimum water content of the total material, percent } \\
\mathrm{P}_{\mathrm{g}} & =\text { decimal value of percent gravel in the total material }
\end{aligned}
$$

97. Figures 81 and 82 present the Optimum Water Content Factors $F_{\text {opt }}$ based on the minus No. 4 and minus $3 / 4-i n$. fractions calculated for the compaction data of Torrey and Donaghe plotted versus gravel content in the total materials. As was the case for the Density Interference Coefficients, the Optimum Water Content Factors also yield a smooth curve with gravel content. However, unlike the Density Interference Coefficients obtained by Torrey and Donaghe, both Figures 81 and 82 show that the optimum water content factors based on the minus No. 4 fraction and those based on the minus 3/4-in. 
fraction represent separate data sets. This is attributed to the significant shift in the compaction parameters with addition of small quantities of gravel to a minus No. 4 material as previously pointed out in discussion of maximum permissible degrees of scalping. Also, it is clear from Figures 81 and 82 that plasticity of fines (minus No. 200 sieve sizes) influences the Optimum Water Content Factor much more so than the Density Interference Coefficient. Figures 83 and 84 present the data of Figures 81 and 82 plotted in $\log -\log$ coordinates where it is seen that the data tends toward linearity in that plot space although there are significant deviations and the linear $\log \log$ fit is not of the quality seen in Figures 81 and 82 for the cartesian curvilinear fit.

98. Figure 85 presents the Optimum Water Content Factors calculated from the data reported by the previously referenced investigators. This figure confirms the smooth trend in the factor with gravel content for each investigator's data set. Figure 86 presents these factors plotted in $\log -\log$ coordinates. In general, the linear $\log -\log$ fittings to the data of the other investigators is better than that seen for the WES data in Figures 83 and 84 . It is also of interest that the slopes of the best-fit linear relationships of Figure 86 are very similar with the exception of the Garga and Madureira data. Garga and Madureira did not directly determine the optimum water contents of compacted specimens of total material by oven drying because of the lack of large capacity equipment. Instead, they calculated those water contents using Equation 4 with the water content of the fraction and an assumed value of water content of the gravel. The authors suspect that this practice may explain the difference in slope of their data seen in Figure 86. Also, it is not readily apparent in Figure 86 , but it is important to note from the data of Donaghe and Townsend (1975) that the Optimum Water Content Factor corresponding to their total material containing 60 percent gravel shows a reversal of curvature compared to lower gravel contents. It may be true that just as for the Density Interference Coefficient, the Optimum Water Content Factor coefficient will deviate from approximate linearity in $\log -\log$ coordinates at some higher gravel content. 
Predicting the optimum water

content of the total material

using Optimum Water Content Factors

99. Given the optimum water content of either the minus No. 4 or minus 3/4-in. fraction, the gravel content in the total material, and the corresponding value of the Optimum Water Content Factor $F_{\text {opt }}$ it is a simple matter to predict the optimum water content of the total material using Equation 12. Figures 87 and 88 provide the results of predictions of optimum water content of the total materials using Optimum Water Content Factors obtained by Torrey and Donaghe based on the minus No. 4 and minus 3/4-in. fractions, respectively. The Optimum Water Content Factors were picked off the curves fit by eye to the $F_{\text {opt }}$ versus gravel content in the total material data of Figures 81 and 82 . It was found that plotting of the data to a scale permitting estimation of $F_{\text {opt }}$ to the second decimal place was sufficient. In like manner, Figure 89 presents predictions of optimum water content for the various referenced previous investigators' materials employing estimated-fit $F_{\text {opt }}$ curves to the data of Figure 85 . It is to be noted that smooth curves could be fit precisely through each data point derived from the USBR and Donaghe and Townsend of Figure 85 . This resulted in precise predictions of optimum water contents of their total materials.

100. Figure 90 is intended to indicate the sensitivity of the predicted value of optimum water content to variation in the Optimum Water Content Factor $F_{\text {opt }}$. To accomplish this, a fixed value of 13 percent was used for the optimum water content of the fraction. This value is approximately the average value for the minus No. 4 fractions containing clay fines from among the various investigators. As was the case for the Density Interference Coefficient $I_{c}$ (see Figure 72), Figure 90 shows that the sensitivity of the predicted value of optimum water content with change in Optimum Water Content Factor $F_{\text {opt }}$ increases with increasing gravel content in the total material.

Developing Optimum Water Content

Factors without large-scale compaction tests on the total material

101. If one accepts the adequacy of the linearity of the Optimum Water Content Factor versus gravel content of the total material curve in $\log -\log$ coordinates, a similar procedure to that described previously for obtaining the Density Interference Coefficient curve without large-scale testing of the total material can be employed. In this case, the Optimum Water Content 
Factors obtained will be based on the minus No. 4 fraction. Again it is necessary that the minus $3 / 4-i n$. fractions of the total materials span a sufficient range in their own gravel contents. The reader is referred back to paragraph 95 for the fundamentals of the procedure which are the same for the Optimum Water Content Factor. The data presented herein indicates that assumption of linearity of the $F_{\text {opt }}$ versus gravel content curve in $\log -\log$ coordinates should probably not be taken above a gravel content in the total material of about 50 percent as was the case for the Density Interference Coefficient $I_{c}$.

102. The authors have presented new methods for predicting the maximum dry density and optimum water content of a total material from tests performed on a fraction for materials containing maximum particle size up to 4-in. (Gordon, Hammond, and Miller (1964) tested this maximum size). Those predictions are based on two new parameters termed the Density Interference Coefficient and the Optimum Water Content Factor as previously defined. Their relative numerical values are shown in Figures 91 and 92 for the materials tested by Torrey and Donaghe. The authors are convinced that the data obtained from the companion investigation by Torrey and Donaghe and that from other cited investigators support the feasibility of the new methods as long as they are applied to adequately defined families of compaction curves. This is only the same requirement applicable to other methods in use. Since the techniques have been judged on the basis of compaction curves which were established in a conventional manner with absolutely no gerrymandering thereafter, it is reasonable to believe that the results reported can be achieved by USACE division and field laboratories. The values of maximum dry density and optimum water content are subjective judgments, i.e., a compaction curve must be fitted by individual judgment to data points usually exhibiting some scatter, and several versions of large-scale compaction equipment/procedures have been employed. Consequently, the authors suspect that the fitted curves of Density Interference Coefficient $I_{c}$ and Optimum Water Content Factor $F_{\text {opt }}$ versus gravel content in the total material may yield estimates of maximum dry density and optimum water content of a total material as good as or better than any other approach. The proof will lie in the application of the new methods in actual project situations including the treatment of materials with maximum particle size in excess of $4 \mathrm{in}$. 


\section{Applying the Density Interference Coefficient and Optimum Water Content Factor in Compaction Control}

103. Once the curves of Density Interference Coefficient $I_{c}$ and Optimum Water Content Factor $F_{\text {opt }}$ versus gravel content have been developed, compaction control of the fill can be based only on the one- or two-point field compaction procedure applied to either the minus $3 / 4-i n$. sieve or minus No. 4 sieve fractions of the fill density samples. As will be explained below, it is most efficient to base control on the minus No. 4 fraction. To obtain the maximum dry density of the fill sample, the following procedure can be used assuming that the bulk specific gravity $G_{m}$ of the gravel has been properly determined.

a. Determine the fill dry density $\gamma_{t}$ and water content $w_{t}$ of the total fill sample by the appropriate fill density test.

b. From the fill density test sample plus any additional grab sample from the location of the fill density sample as needed to provide sufficient material, determine the percent gravel $\mathrm{P}_{\mathrm{g}}$ the percent oversized fraction $c$ and the percent finer fraction $f$.

c. From the curves of Density Interference Coefficient and Optimum Water Content Factor versus gravel content, pick off the values of $I_{c}$ and $F_{o p t}$ which correspond to the gravel content of the total fill sample $P_{B}$.

d. Determine the maximum dry density of the finer fraction $\gamma_{\text {fmax }}$ and its optimum water content $W_{\text {fopt }}$ by the one- or two-point compaction method based on either the minus $3 / 4-i n$. or minus No. 4 sieve fraction of the total fill density material. This, of course, presumes that the appropriate family of compaction curves has been developed pertaining to either the minus $3 / 4-i n$. or minus No. 4 sieve fractions of the total materials to be placed in the fill (see paragraph 25). Note that it is more efficient to use the minus No. 4 fraction because percent oversize $c$ and percent gravel in the total material $\mathrm{P}_{\mathrm{g}}$ are the same number. This eliminates an extra sieving operation which would be required if $\gamma_{f \max }$ and $W_{\text {fopt }}$ are for the minus $3 / 4-i n$. fraction since both the percent oversize (plus $3 / 4-i n$. material) and the percent gravel in the total material would have to be determined.

e. To determine the maximum dry density corresponding to the gradation of the total fill sample $\gamma_{\text {tmax }}$ enter Equation 11, which is,

$$
\gamma_{t \max }=\frac{P_{g} I_{c} \gamma_{f \max } \gamma_{w} G_{m}}{f \gamma_{w}+P_{g} C I_{c} \gamma_{f \max }}
$$


with the value for $\gamma_{f \max }$ determined by the one- or two-point method in $\underline{d}$. above and the values for $I_{c}, f, c, G_{m}, P_{g}$, and $\gamma_{w}$ and solve for $\gamma_{\text {tmax }}$. Calculate the percent compaction of the total fill sample, i.e., $\gamma_{t} / \gamma_{\text {tmax }}$ and compare that value to the desired percent compaction.

f. Equation 12 for Optimum Water Content Factor can be rearranged to solve for the optimum water content of the total material $\mathrm{W}_{\text {topt }}$ as follows:

$$
W_{\text {topt }}=\frac{W_{\text {fopt }}}{P_{B} F_{\text {opt }}}
$$

Enter the value for $W_{\text {fopt }}$ determined by the one- or two-point method in $\underline{\mathrm{d}}$. above and the value for $F_{\text {opt }}$ from $\underline{c}$. above and the value of $\mathrm{P}_{g}$ and solve Equation 12) for the optimum water content of the total material $W_{\text {topt. }}$. Determine the wet or dry deviation of the water content of the total fill sample $W_{t}$ from its optimum water content $W_{\text {topt }}$ and compare that deviation to the specified range. 


\section{PART VII: CONCLUSIONS AND RECOMMENDATIONS}

\section{Conclusions}

104. The following conclusions are drawn concerning current procedures for laboratory determination of the maximum dry density and optimum water content of soils containing large particles:

a. There is no widely accepted standardized large-scale compaction test for soils containing large particles.

b. The current compaction test for earth-rock mixtures of USACE (1970), Appendix VIA, is limited to a maximum particle size of $2-i n$. and requires the use of a $11.5 \mathrm{lb}$ hand-held rammer. It is a laborious and time consuming test which has typically drawn criticism from those who have employed it.

c. A large-scale compaction test utilizing a mechanical compactor and a variety of mold diameters which is satisfactorily free of equipment size effects for soils containing gravel has been developed (Torrey and Donaghe 1991). However, that test will not replicate results on minus No. 4 sieve material obtained in the 4-in. diameter mold with the hand-held rammer.

d. The current practice of USACE (1970) allowing the scalping of up to 5 percent by weight of oversized gravel particles to permit performance of the compaction test in a smaller mold may or may not yield values of maximum dry density and optimum water content which are satisfactorily representative of the unaltered total gradation. The higher the gravel content of the total gradation, the more likely that this scalping criteria will be satisfactory. The data obtained by Torrey and Donaghe (1991) for gradations containing both silt (ML) and clay ( $\mathrm{CH}$ ) fines (minus No. 200 sieve sizes) indicated that the 5 percent scalping rule would suffice based on a precision of two percent of the mean value of maximum dry density and ten percent of mean value of optimum water content for total materials containing at least 15 percent gravel. At gravel contents lower than about 15 percent, 5 percent scalping may result in compaction parameters which fall outside a precision range of two percent of the mean value of maximum dry density and 10 percent of the mean value of optimum water content of the unaltered gradation. With increasing gravel content in the total material above about 15 percent, the acceptable percent scalping also increases above 5 percent.

e. Gradations with a smaller maximum particle size generated from a total material by the scalping with replacement procedure cannot be expected to yield values of maximum dry density and optimum water content which are equivalent to those of the parent total material. The scalped and replaced gradation will exhibit a lower maximum dry density and higher (wetter) optimum water content than the parent total material. The 
larger the relative proportion of the material scalped and replaced and the greater the reduction in maximum particle size, the more the compaction parameters of the scalped and replaced gradation will differ from those of the parent total material.

105. The following conclusions are drawn relative to methods for correcting dry density of a total material to obtain the corresponding value for a fraction and for calculating the maximum dry density of a total material from the corresponding value for a fraction.

a. Ziegler's Equation 1 as follows (see paragraph 48),

$$
\gamma f=\frac{f \gamma_{t} \gamma_{w} G_{m}}{\gamma_{w} G_{m}-C \gamma_{t}}
$$

is valid for determining the dry density of a fraction given the dry density of the total material containing gravel as long as the gradation of the total material is such that the fraction completely fills the space surrounding or between the large particles. This condition on the applicability of the equation can generally be assumed to be met up to gravel contents of 60 percent.

b. When Ziegler's Equation 1 is rearranged in terms and modified as follows (see paragraph 50 ),

$$
\gamma_{\mathrm{tmax}}=\frac{\gamma_{\mathrm{fmax}} \gamma_{\mathrm{w}} G_{\mathrm{m}}}{f \gamma_{\mathrm{w}} G_{\mathrm{m}}+C \gamma_{\mathrm{fmax}}}
$$

and used to predict the maximum dry density of the total material by insertion of the value of maximum dry density of the fraction, the approximation 3 is no longer an equation. The accuracy of the estimate of the value of maximum dry density of the total material obtained from expression 3 is dependent upon the gradation of the total material, the plasticity of the fines (minus No. 200 sieve sizes), and the fraction, i.e., the minus No. 4 or minus 3/4-in., employed. USACE (1977), Appendix B, states that approximation 3 may be considered sufficiently accurate up to a gravel content of 35 percent in the total material. However, careful review of the literature and recent findings by Torrey and Donaghe (1991) indicate that some materials containing less than 35 percent gravel may not be treated satisfactorily using approximation 3. Furthermore, use of the approximation 3 may seriously overpredict the maximum dry density of total materials containing low gravel contents which would lead to inflated values of in place percent compaction for such gradations.

c. The modification of Ziegler's Equation 1 to account for the 
actual percent compaction of the fraction when the total material is at its maximum dry density by incorporation of a Fraction Density Factor $R_{c}$ as follows (see paragraph 63),

$$
\gamma_{\mathrm{tmax}}=\frac{R_{\mathrm{c}} \gamma_{\mathrm{fmax}} \gamma_{\mathrm{w}} G_{\mathrm{m}}}{f \gamma_{\mathrm{w}} G_{\mathrm{m}}+R_{\mathrm{c}} C \gamma_{\mathrm{fmax}}}
$$

does not alter the validity of the equation as long as the conditions cited in a. above are met and the correct value of $R_{c}$ is inserted. The Fraction Density Factor $R_{c}$ is the percent compaction of the fraction, i.e., $\gamma_{f} / \gamma_{\text {fmax }}$, expressed as a decimal when the total material is at its maximum dry density. The USBR and the AASHTO employ this method and refer to $R_{c}$ as the "rock correction factor". The Fraction Density Factor $R_{c}$ is a function of gradation and plasticity of fines. It has been shown to vary over a significant range as a function of gravel content. Therefore, there is no single curve or narrow band relating $R_{c}$ and gravel content which can suffice for general usage since small variations in the value of $R_{c}$ have a significant impact on the value of maximum dry density of the total material calculated using Equation 5 above.

d. The equation (see paragraph 64 ),

$$
\gamma_{\mathrm{t} \max }=\frac{1-(0.05) F}{\frac{F}{162}+\frac{1-F}{\gamma_{f \max }}}
$$

offered by US Department of the Navy (1982) to calculate the maximum dry density of the total material from that of a fraction is tantamount to a single curve of Fraction Density Factor $R_{c}$ versus gravel content. Therefore, Equation 6 cannot be considered reliable for the reasons cited in $\underline{b}$. above.

e. Ziegler's Equation 1 may be modified to account for the actual percent compaction of the fraction when the total material is at its maximum dry density by incorporation of a Density Interference Coefficient $I_{c}$. The Density Interference Coefficient is defined as the Fraction Density Factor $R_{c}$ divided by the product of the percent gravel (expressed as a decimal) in the total material $P_{8}$ times the bulk specific gravity of the gravel $G_{m}$ i.e., $I_{c}=R_{c} / P_{g} G_{m}$ (see page 68 ). The modified equation becomes (see paragraph 89 ):

$$
\gamma_{\mathrm{t} \max }=\frac{P_{\mathrm{g}} I_{\mathrm{c}} \gamma_{\mathrm{fmax}} \gamma_{\mathrm{w}} G_{\mathrm{m}}}{f \gamma_{\mathrm{w}}+P_{\mathrm{g}} C I_{\mathrm{c}} \gamma_{\mathrm{fmax}}}
$$

The accuracy of Equation 11 is dependent on insertion of the correct value for the Density Interference Coefficient $I_{c}$. Unlike the Fraction Density Factor $R_{c}$ the Density 
Interference Coefficient $I_{c}$ has been shown to fall on a single curve versus gravel content for entire families of generically similar gradations and their fractions which contain gravel whether the coefficient is based on $R_{c}$ for the minus $3 / 4-i n$, or minus No. 4 fraction. By generically similar gradations, it is meant that the gravel fractions, sand fractions and fines (minus No. 200 sieve sizes) are consistent in their mineralogy, grain shapes, plasticity, etc., as would be expected from materials obtained from a single borrow source or possibly even multiple borrow sources within a given geological environment.

f. When the curve of Density Interference Coefficient $I_{c}$ versus gravel content was plotted in $\log -\log$ coordinates it became a straight line (see paragraph 95) over the range in gravel content between about 10 percent and 45 percent for those gradations tested by Torrey and Donaghe (1991). The data from other investigators falling within this range in gravel content also displayed linearity. For all data examined, above about 45 percent gravel the curves were no longer linear in log-log coordinates but appeared to be linear in cartesian coordinates. When the Density Interference Coefficient as defined by the equation given in $\underline{e}$. above is based on the minus No. 4 fraction, $I_{c}$ becomes very large and approaches infinity as $P_{g}$ approaches zero. When $I_{c}$ is based on the minus 3/4-in. fraction, it approaches a maximum value as the gravel content in the total material approaches the gravel content of the minus $3 / 4-i n$. fraction, 1 ,e., as the percent oversize, c, approaches zero.

g. The linearity of the Density Interference Coefficient $I_{c}$ versus gravel content in the total material in $\log -\log$ coordinates may be used to establish the total curve without testing the total material which requires large-scale testing equipment. This can be achieved if the minus $3 / 4-i n$. fractions of the total materials contain a sufficient range in gravel content by basing $I_{c}$ on the minus No. 4 fraction and treating the minus $3 / 4-i n$. fraction as a total material. The procedure is described in paragraph 95.

h. If the curve of Density Interference Coefficient $I_{c}$ versus gravel content is available for a given family of generically similar gradation curves, the maximum dry density of a fill sample of the total material can be calculated from Equation 11 given in e. above using the value of $I_{c}$ picked from the curve at the gravel content in the fill sample. To accomplish this, it is best to plot the $I_{c}$ versus gravel content in the total material curve in cartesian coordinates to a scale convenient for picking off a value of $I_{c}$ to the third decimal place. If the curve of $I_{c}$ versus gravel content has been determined as in $\mathrm{g}$. above, it should be converted to the cartesian coordinate form. It has been shown herein, that this procedure is practical and that estimates of the maximum dry density of the total material thus obtained are precise within two percent of the mean value of maximum dry 
density. This precision is as good or better than that which would be obtained if the value of maxinum dry density of the total material were determined by replicate testing in the same laboratory by two experienced tectnicians.

106. The following conclusions are drawn relative to methods for correcting the water content of the total material to obtain that of a fraction and for calculating the optimum water content of a total material from the corresponding value for a fraction.

a. The following equation (see paragraph 48),

$$
W_{f}=\frac{W_{t}-C W_{c}}{f}
$$

is a weight-volume relationship which must be true. USACE (1977), Appendix B, gives this equation except that the absorption of the gravel, A, is substituted for the water content of the coarser (oversized) fraction $W_{c}$. This modification is used to avoid determination of the water content of the total material for each fill sample which would require large samples and large capacity ovens. The accuracy of the modification depends upon how close the value of the water content of the oversized fraction (gravel) is to its absorption, A, which reflects the saturated surface-dry condition.

b. When Equation 2 is rearranged to solve for the water content of the total material $W_{t}$ it becomes (see paragraph 50 ),

$$
W_{t}=f w_{f}+C W_{c}
$$

When this version of the equation is modified to estimate the optimum water content of the total material $W_{\text {topt }}$ from that of a fraction $W_{\text {fopt }}$ as follows (see paragraph 50 ),

$$
W_{\text {topt }}=f W_{\text {fopt }}+C W_{c}
$$

it becomes an approximate relationship. It is not feasible to state a gravel content in the total material up to which this approximation will be generally acceptable because that range in gravel content is a function of gradation and plasticity of fines.

c. In lieu of Equation 4, the optimum water content of the fraction $W_{\text {fopt }}$ can be directly related to that of the total material $W_{\text {topt }}$ and the gravel content of the total material $\mathrm{P}_{\mathrm{g}}$ by an Optimum Water Content Factor $\mathrm{F}_{\text {opt }}$ defined as follows (see paragraph 97): 


$$
F_{\text {opt }}=\frac{\frac{W_{\text {fopt }}}{W_{\text {topt }}}}{P_{g}}
$$

As is the case for the Density Interference Coefficient, $I_{c}$, the Optimum Water Content Factor $F_{\text {opt }}$ has been shown to conform to a single curve versus gravel content of the total material for a family of generically similar gradations and their fractions which contain gravel.

d. When the Optimum Water Content Factor $F_{\text {opt }}$ versus gravel content in the total material curve is plotted in $10 \mathrm{~g}-\log$ coordinates, it becomes a straight line over a significant range in gravel content. For the data examined in this report, that range was from 10 to as much as 64 percent gravel, but it appears necessary to prove linearity above a gravel content of 50 percent for any given family of generically similar gradations since some of the data examined deviated from linearity above that approximate gravel content.

e. The linearity of the Water Content Factor $F_{\text {opt }}$ versus gravel content in the total material in $\log -\log$ coordinates may be used to establish the total curve without testing the total material which requires large-scale testing equipment. This can be achieved if the minus 3/4-in. fractions of the total materials contain a sufficient range in gravel content by bas ing $F_{\text {opt }}$ on the minus No. 4 fraction and treating the minus 3/4-in. fraction as a total material. The procedure is described in paragraph 102.

f. If the curve of Optimum Water Content Factor $F_{\text {opt }}$ versus gravel content is available for a given family of generically similar gradation curves, the optimum water content of a fill sample of the total material can be calculated from Equation 12 given in $\underline{c}$. above using the value of $F_{\text {opt }}$ picked from the curve at the gravel content in the fill sample. To accomplish this, it is best to plot the $F_{\text {opt }}$ versus gravel content in the total material curve in cartesian coordinates to a scale convenient for picking off a value of $F_{\text {opt }}$ to the third decimal place. If the curve of $F_{\text {opt }}$ versus gravel content has been determined as in e. above, it should be converted to the cartesian coordinate form. It has been shown herein, that this procedure is practical and that estimates of the optimum water content of the total material thus obtained are precise within ten percent of the mean value of optimum water content. This precision is as good or better than that which would be obtained if the value of optimum water content of the total material were determined by replicate testing in the same laboratory by two experienced technicians . 
Recommendations

107. The following recommendations are offered relative to the current procedures for laboratory determination of the maximum dry density and optimum water content of soils containing large particles:

a. It is recommended that the large scale compaction test developed by Torrey and Donaghe (1991) for a mechanical compactor and $6-, 12-$ and $18-i n$. diameter molds be adopted for inclusion in USACE (1970) as a substitution for the current Appendix VIA, "Compaction Test for Earth-Rock Mixtures." It is obviously very desirable to employ compaction control procedures which do not require the routine compaction of earth-rock mixtures necessitating the use of such large-scale equipment and ancillary facilities. However, based on findings presented in this report and those reported in the earlier companion report by Torrey and Donaghe (1991), it is indicated that there will arise occasions in which anticipated compaction control procedures will require verification for the specific materials involved during the design phase of the project and perhaps occasional checks during construction. For this reason, the new mechanical compaction test method has its place in the Engineer Manual. It is pointed out that the USBR has had such a mechanical large-scale procedure in its Earth Manual for many years. However, unlike the USBR procedure, the new method offered by Torrey and Donaghe (1991) was developed with attention to elimination of equipment size effects among the three mold sizes (6-, 12- and 18-in. diameter).

b. The question of maximum allowable scalping should be revisited by the USACE perhaps via the Division Laboratory Conference.

c. It is recommended that the scalping with replacement procedure be discontinued.

108. The following recommendations are made relative to methods for correcting dry density of a total material to obtain the corresponding value for a fraction and for calculating the maximum dry density of a total material from the corresponding value for a fraction:

a. The basic Ziegler's Equation 1 (see conclusion 105.a.) should be used to calculate the dry density of a fraction given the dry density of a total material. Equation 1 should not be routinely assumed to be accurate for materials containing more than about 60 percent gravel. As gravel content reaches and exceeds such high levels, the conditions upon the accuracy of the equation as discussed in paragraph 51 are no longer met.

b. The modification of Ziegler's Equation 1 to the approximate relationship 3 (see conclusion 105.‥) for the purposes of estimating the maximum dry density of the total material from that of a fraction may be used if it is verified by testing that it is sufficiently accurate for both the lower and higher 
limits of the range in gravel contents of the total materials to be placed in the fill. The current suggestion in USACE (1977), Appendix B, that this method is generally applicable to materials containing up to 35 percent gravel is not reliable.

c. The modification of Ziegler's Equation 1 to obtain Equation 5 (see conclusion 105.‥) by inclusion of the Fraction Density Factor $R_{c}$ to account for the actual dry density of the fraction when the total material is at it maximum dry density, is recommended as an acceptable method for predicting the maximum dry density of the total material from that of a fraction. That recommendation is contingent upon the determination by testing of the correct values of $R_{c}$ versus gravel content for the range of total materials at hand. Generic curves or narrow bands of $R_{c}$ versus gravel content such as those offered by USBR and AASHTO should not be used unless proven to be applicable. The shapes of the $R_{c}$ versus gravel content relationships cited by USBR and AASHTO conform to the similar trends seen for a wide range in earth-rock gradations and, therefore, may be applicable to certain materials.

d. The Equation 6 (see conclusion 105.‥) offered by the Department of the Navy (1982) is not recommended as a means of estimating the maximum dry density of a total material from that of a fraction. That equation is equivalent to a single curve of Fraction Density Factor $R_{c}$ versus gravel content. Furthermore, the shape of the equivalent $R_{c}$ versus gravel content curve does not correspond to the similar trends seen for a wide range in earth-rock gradations (see Figure 49) which suggests that Equation 6 is not a valid relationship.

e. It is recommended that the new modification to Ziegler's Equation 1 to the form of Equation 11 (see conclusion 105.e.) which includes the Density Interference Coefficient $I_{c}$ be assessed by application to a major USACE project. The compaction control procedure given in paragraph 104 is recommended. That effort should include assessment of the establishment of the $I_{c}$ versus gravel content curve for the entire family of earth-rock gradations without testing of the total materials as described herein in paragraph 94. Only very limited compaction testing of selected total materials would be required to confirm the shortcut approach. The few compaction tests on total materials could be conducted by another Division Laboratory with large-scale capability or the WES should those capabilities be lacking within the Division responsible for the project.

109. The following recommendations are made relative to methods for correcting the water content of the total material to obtain that of a fraction and for calculating the optimum water content of a total material from the corresponding value for a fraction.

a. Equatior 2 (see conclusion 106 , a.) is recommended for calculating the water content of a fraction $W_{f}$ given that of the 
total material and that of the oversized fraction $W_{c}$. The equation can be rearranged to solve for the water content of the total material $\mathrm{W}_{\mathrm{t}}$ (see conclusion 106.…). Care should be exercised in substituting the absorption of the gravel A for the water content of the oversize fraction. It is not a prohibitive undertaking to determine a more correct general value for water content of the oversize fraction as described in Appendix B to this report.

b. When Equation 2 is modified to obtain expression (4) (see conclusion 106 . total material $W_{\text {topt }}$ it becomes an approximate expression. The range in gravel content of the total material over which this approximate expression is sufficiently accurate is a function of gradation and plasticity of fines. It is recommended that this approach not be used unless the adequacy of the approximation is confirmed for the range of materials at hand.

c. It is recommended that the new approach to estimating the optimum water content of the total material employing the Optimum Water Content Factor $F_{\text {opt }}$ (see conclusion 106.c.) be employed. The compaction control procedure given in paragraph 104 is recommended. The relationship of $F_{\text {opt }}$ versus gravel content in the total material is linear when plotted in loglog coordinates and can be determined for the entire family of gradations of the total material possibly without large-scale testing as discussed in paragraph 101. This approach does not involve a water content for the oversized fraction as it directly relates the optimum water content of the total material to that of a fraction. 
American Society for Testing and Materials. 1991a. "Standard Test Methods for Moisture-Density Relations of Soils and Soil-Aggregate Mixtures Using 5.5-1b Rammer and 12-in. Drop," Designation D 698-78, 1991 Annual Book of ASTM Standards, Vol 04.08, ASTM, Philadelphia, PA, pp 160-164.

1991b. "Standard Test Methods for Moisture-Density Relations of Soils and Soil-Aggregate Mixtures Using 10-1b Rammer and 18-in. Drop," Designation D 1557-78, 1991 Annual Book of ASTM Standards. Vol 04.08, ASTM, Philadelphia, PA, Pp 217-221.

1991c. "Standard Practice for Use of Terms Precision and Bias in ASTM Test Methods," Designation E 177-86, 1991 Annual Book of ASTM Standards. Vol 14.02, ASTM, Philadelphia, PA, pp 89-99.

1991d. "Standard Test Method for Specific Gravity and Absorption of Coarse Aggregate," Designation C 127-88, 1991 Annual Book of ASTM Standards. Vol 04.02, ASTM, Philadelphia, PA, pp 63-67

. 1991e. "Standard Test Method for Rapid Determination of Percent Compaction," Designation D 5080-90, 1991 Annual Book of ASTM Standards. Vol 04.08, ASTM, Philadelphia, PA.

Donaghe, R. T. and Townsend, F. C. 1973. "Compaction Characteristics of Earth-Rock Mixtures, Report 1, Vicksburg Silty Clay and DeGray Dam Clayey Sandy Gravel," Miscellaneous Paper S-73-25, US Army Engineer Waterways Experiment Station, Vicksburg, MS .

1975. "Compaction Characteristics of Earth-Rock Mixtures, Report 2, Blended Material," Miscellaneous Paper S-73-25, US Army Engineer Waterways Experiment Station, Vicksburg, MS.

Donaghe, R. T. and Torrey, V. H. 1985. "Strength and Deformation Properties of Earth-Rock Mixtures," Technical Report GL-85-9, US Army Engineer Waterways Experiment Station, Vicksburg, MS .

Garga, V. K. and Madureira, C. J. 1985. "Compaction Characteristics of River Terrace Gravel," Journal of Geotechnical Engineering. ASCE, Vol 111, No. 8.

Gordon, B. B., Hammond, W. D., and Miller, R. K. 1964. "Effect of Rock Content on Compaction Characteristics of Clayey Gravel," Compaction of Soils. Special Technical Publication No. 377, ASTM, pp 31-46.

Hammer, D. P. and Torrey, V. H. 1973. "Test Fills for Rock-Fill Dams," Miscellaneous Paper S-73-7, US Army Engineer Waterways Experiment Station, Vicksburg, MS.

Hammitt, G. M. 1966. "Statistical Analysis of Data from a Comparative Laboratory Test Program Sponsored by ACIL," Miscellaneous Paper 4-785, US Army Engineer Waterways Experiment Station, Vicksburg, MS.

McLeod, N. W. 1958. "Suggested Method for Correcting Maximum Density and Optimum Water Content of Compacted Soils for Oversize Particles, " Procedures for Testing Soil. Third Edition, American Society for Testing and Materials, Philadelphia, PA, pp 143-145. 
Sherwood, P. T. 1970. "The Reproducibility of the Results of Soil Classification and Compaction Tests," Report LR 339, Road Research Laboratory, Earthworks and Foundations Section, Ministry of Transport, Crowthorne, Berkshire, England.

Strohm, W. E. 1966. "Preliminary Analysis of Results of Division Laboratory Tests on Standard Soil Samples, "Miscellaneous Paper 3-813, US Army Engineer Waterways Experiment Station, Vicksburg, MS.

Strohm, W. E. and Torrey, V. H. 1982. "Analysis of Field Compaction Data, DeGray Dam, Caddo River, Arkansas," Miscellaneous Paper GL-82-4, US Army Engineer Waterways Experiment Station, Vicksburg, MS.

Torrey, V. H. 1970a. "Analysis of Field Compaction Data, Report 1, Perry Dam, Delaware River, Kansas," Miscellaneous Paper S-70-13, US Army Engineer Waterways Experiment Station, Vicksburg, MS.

1970b. "Analysis of Field Compaction Data, Report 2, Littleville Dam, Westfield River, Massachusetts," Miscellaneous Paper S-70-13, US Army Engineer Waterways Experiment Station, Vicksburg, MS.

1970c. "A Statistical Approach to the Problem of Compaction Control of Major Earth-Fill Structures," Master's Thesis, Mississippi State University, State College, MS.

Torrey, V. H. and Donaghe, R. T. 1991. (in preparation) "Compaction Characteristics of Earth-Rock Mixtures," Technical Report GL-90- , US Army Engineer Waterways Experiment Station, Vicksburg, MS.

US Army Corps of Engineers. 1977. "Construction Control for Earth and RockFill Dams," Engineer Manual EM 1110-2-1911, Headquarters, Department of the Army, Office Chief of Engineers, Washington, DC.

- 1970. "Laboratory Soils Testing," Engineer Manual EM 1110-2-1906, Headquarters, Department of the Army, Office Chief of Engineers, Washington DC.

US Bureau of Reclamation. 1963a. Earth Manual, Denver, CO.

. 1963b. "Research on Compaction Control Testing for Gravelly Soils,"

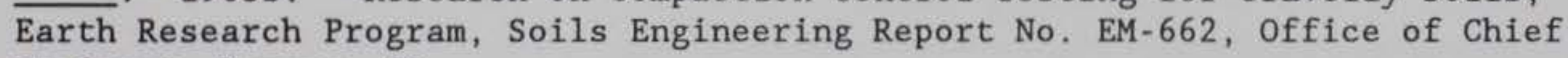
Engineer, Denver, CO.

1989a. "Procedure for Performing Laboratory Compaction of Soils Containing Grave1," Method USBR 5515-89, Denver, CO.

1989b. "Performing Rapid Method of Construction Control, " Method USBR 7240-89, Denver, CO.

US Department of the Navy. 1982. "Foundations and Earth Structures," Design Manual 7.2, Naval Facilities Engineering Command, Alexandria, VA.

Ziegler, E. J. 1948. "Effect of Material Retained on the Number 4 Sieve on the Compaction Test of Soil," Proceedings. Highway Research Board, Vol 28, pp 409-414. 
Table 1

ASTM Designations D $698-78$ and D 1557-78 Precision Standards

\begin{tabular}{|c|c|c|}
\hline$x^{2}$ & $\begin{array}{l}\text { Standard } \\
\text { Deviation } \\
\end{array}$ & $\begin{array}{c}\text { Acceptable Range of } \\
\text { Two Results, Expressed } \\
\text { as Percent of Mean } \\
\text { Value }\end{array}$ \\
\hline \multicolumn{3}{|l|}{ Single-operator precision: } \\
\hline Maximum dry unit weight & $\cdots$ & 1.9 \\
\hline Optimum water content & $\cdots$ & 9.5 \\
\hline \multicolumn{3}{|l|}{ Multilaboratory precision: } \\
\hline Maximum dry unit weight & $\pm 1.66 \mathrm{pcf}$ & 4.0 \\
\hline Optimum water content & $\begin{array}{c} \pm 0.86 \text { percentage } \\
\text { points }\end{array}$ & 15.0 \\
\hline
\end{tabular}


Table 2

Results of Umpire Tests on Standard Soil Samples

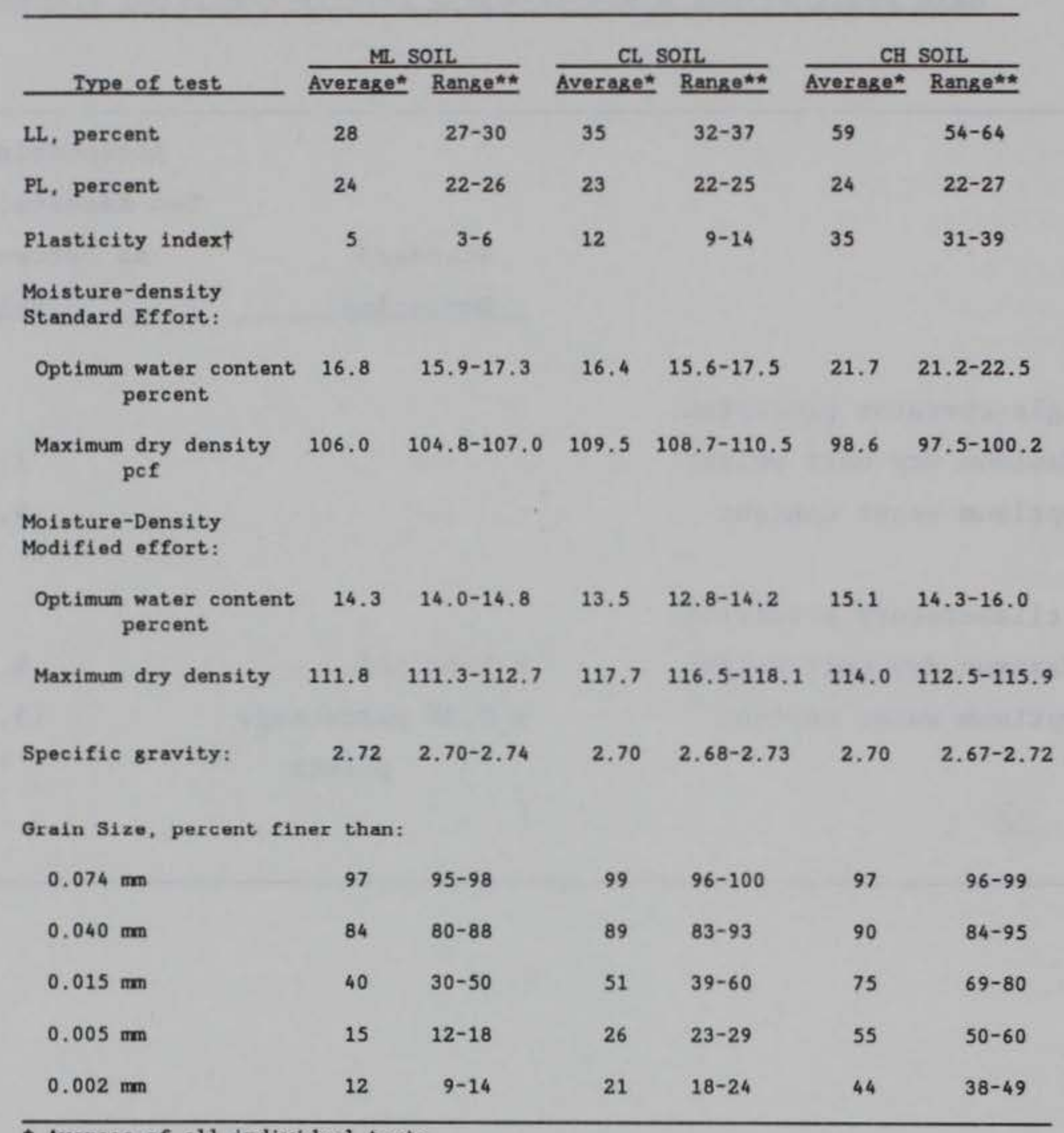

* Average of all individual tests.

** Represents minimum and maximum values of actual test results.

$\dagger$ Ranges are computed from the test values for liquid limit and plastic limit. 
Table 3

Statistical Analysis of ML Soil Considering All Commercial Laboratory Results

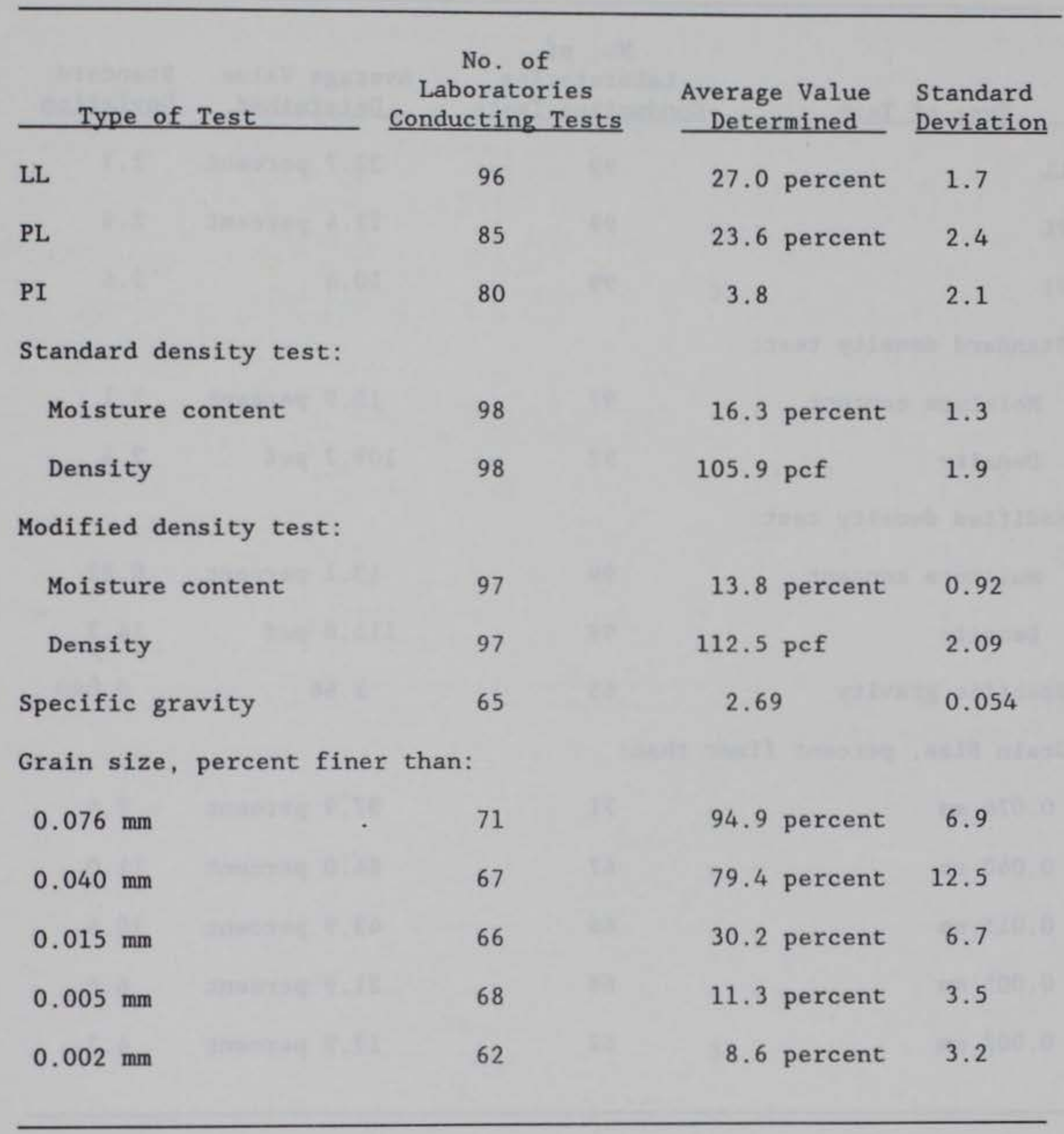


Table 4

Statistical Analysis of CL Soil Considering All Commercial

Laboratory Results

\begin{tabular}{|c|c|c|c|}
\hline Type of Test & $\begin{array}{c}\text { No. of } \\
\text { Laboratories } \\
\text { Conducting Tests } \\
\end{array}$ & $\begin{array}{c}\text { Average Value } \\
\text { Determined } \\
\end{array}$ & $\begin{array}{l}\text { Standard } \\
\text { Deviation }\end{array}$ \\
\hline LL & 99 & 32.7 percent & 2.3 \\
\hline PL & 99 & 22.4 percent & 2.8 \\
\hline PI & 99 & 10.4 & 3.6 \\
\hline \multicolumn{4}{|c|}{ Standard density test: } \\
\hline Moisture content & 97 & 15.9 percent & 1.1 \\
\hline Density & 97 & 109.7 pef & 2.4 \\
\hline \multicolumn{4}{|c|}{ Modified density test: } \\
\hline Moisture content & 99 & 13.1 percent & 0.82 \\
\hline Density & 99 & 115.8 pcf & 14.2 \\
\hline Specific gravity & 65 & 2.66 & 0.060 \\
\hline \multicolumn{4}{|c|}{ Grain Size, percent finer than: } \\
\hline $0.076 \mathrm{~mm}$ & 71 & 97.9 percent & 2.4 \\
\hline $0.040 \mathrm{~mm}$ & 67 & 84.0 percent & 11.0 \\
\hline $0.015 \mathrm{~mm}$ & 66 & 43.9 percent & 10.6 \\
\hline $0.005 \mathrm{~mm}$ & 68 & 21.9 percent & 6.5 \\
\hline $0.002 \mathrm{~mm}$ & 62 & 17.9 percent & 4.7 \\
\hline
\end{tabular}


Table 5

Statistical Analysis of $\mathrm{CH}$ Soil Considering A11 Oommercial.

Laboratory Results

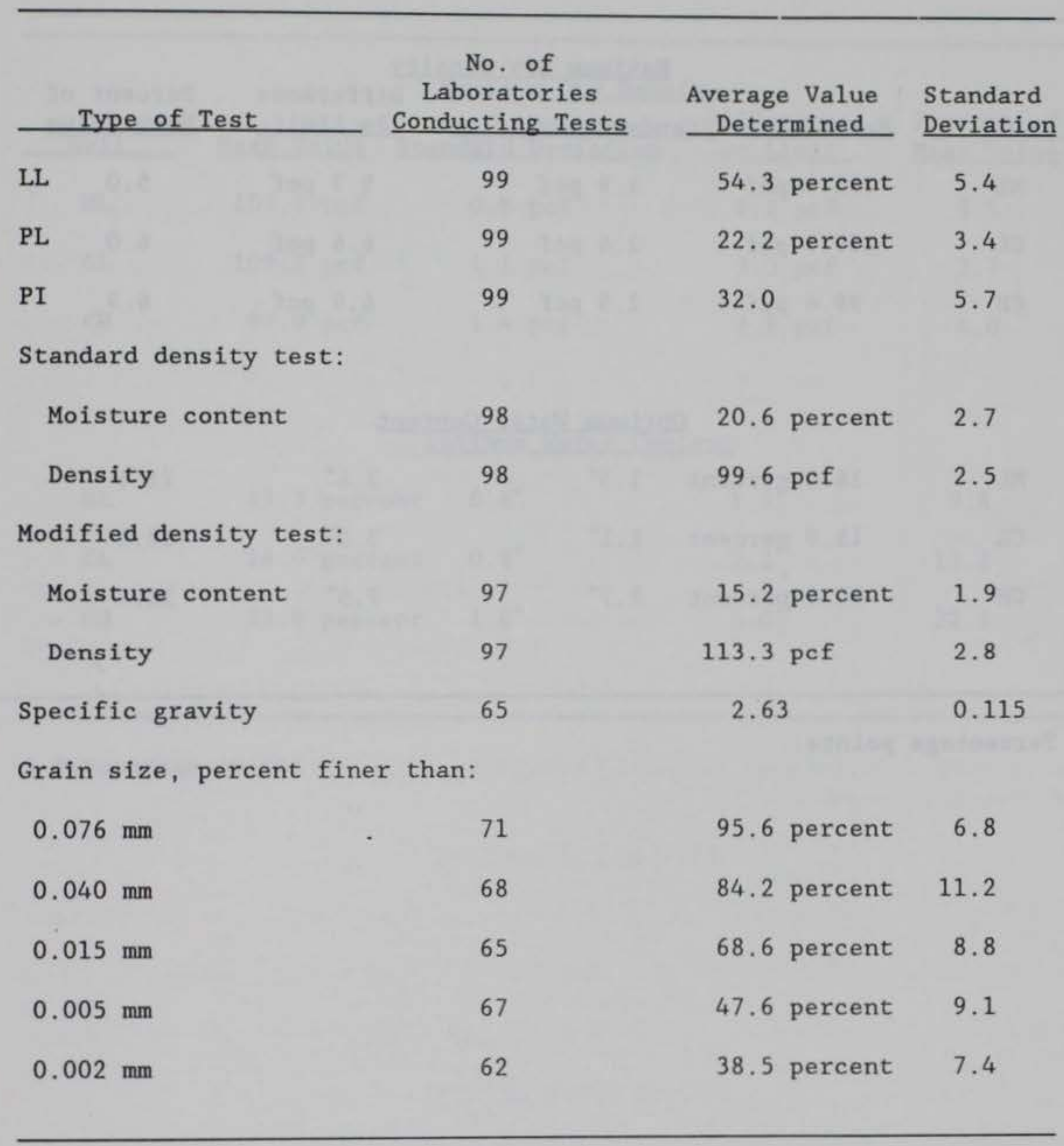


Table 6

Precision Limits for ACIL Study Results

Maximum Dry Density

Difference Percent of

Soil Mean Value Standard Deviation 20 Limit Mean Value

ML

105.9 pcf

1.9 pcf

5.3 pcf

5.0

CL

$109.7 \mathrm{pcf}$

$2.4 \mathrm{pcf}$

$6.6 \mathrm{pcf}$

6.0

$\mathrm{CH}$

$99.6 \mathrm{pcf}$

$2.5 \mathrm{pcf}$

$6.9 \mathrm{pcf}$

6.9

Optimum Water Content

$\begin{array}{lllll}\text { ML } & 16.3 \text { percent } & 1.3^{*} & 3.6^{*} & 22.1 \\ \text { CL } & 15.9 \text { percent } & 1.1^{*} & 3.0^{*} & 18.9 \\ \text { CH } & 20.6 \text { percent } & 2.7^{*} & 7.5^{*} & 36.4\end{array}$

* Percentage points. 
Table 7

Precision Limits for USACE Division Lab Results oll ACIL Standard Soils

Maximum Dry Density

Difference Percent of

Soil Mean Value Standard Deviation 20 Limit Mean Value

ML $\quad 105.7 \mathrm{pcf} \quad 0.8 \mathrm{pcf}$

CL

109.2 pcf

$1.1 \mathrm{pcf}$

$1.4 \mathrm{pcf}$

$97.9 \mathrm{pcf}$

Optimum Water Content

ML

CL

$\mathrm{CH}$
17.3 percent

$0.6^{*}$

16.6 percent

$0.8^{\star}$

22.4 percent

$1.8^{*}$
$1.7^{*}$

9.8

$2.2^{*}$

13.2

5. $0^{*}$

22.3

* Percentage points. 
Table 8

Estimated Precision Limits for ACIL Umpire Lab Results

\begin{tabular}{|c|c|c|c|c|}
\hline \multirow[b]{2}{*}{ Soil } & \multicolumn{3}{|c|}{ Maximum Dry Density } & \multirow[b]{2}{*}{$\begin{array}{l}\text { Percent of } \\
\text { Mean Value }\end{array}$} \\
\hline & Mean Value & $\begin{array}{c}\text { Estimated }{ }^{*} \\
\text { Standard Deviation }\end{array}$ & $\begin{array}{l}\text { Difference } \\
2 \sigma \text { Limit } \\
\end{array}$ & \\
\hline ML & $106.0 \mathrm{pcf}$ & $0.55 \mathrm{pcf}$ & $1.5 \mathrm{pcf}$ & 1.4 \\
\hline $\mathrm{CL}$ & 109.5 pcf & $0.45 \mathrm{pcf}$ & $1.2 \mathrm{pcf}$ & 1.1 \\
\hline $\mathrm{CH}$ & $98.6 \mathrm{pcf}$ & $0.68 \mathrm{pcf}$ & $1.9 \mathrm{pcf}$ & 1.9 \\
\hline
\end{tabular}

Optimum Water Content

$\begin{array}{lllll}\text { ML } & 16.8 \text { percent } & 0.35^{\star \star} & 1.0^{\star \star} & 6.0 \\ \text { CL } & 16.4 \text { percent } & 0.48^{\star \star} & 1.3^{\star \star} & 7.9 \\ \text { CH } & 21.7 \text { percent } & 0.32^{\star \star} & 0.9^{* \star} & 4.1\end{array}$

* Standard deviations estimated by taking range of the data to be $4 \sigma$. ** Percentage points. 
Table 9

British Road Research Laboratory (RRL) Study

Multilaboratory

Maximum Dry Density Standard Difference Two-Sigma Percent of

Soil Deviation Precision Limit

Mean Value

Clayey Sand, CL 111.7 pcf

$1.8 \mathrm{pcf}$

$5.0 \mathrm{pcf}$

4.5

Gault Clay, $\mathrm{CH}$

$99.8 \mathrm{pcf}$

$2.0 \mathrm{pcf}$

5.5 pcf

5.5

Weald Clay, $\mathrm{CH} \quad 103.6$ pcf

$2.1 \mathrm{pcf}$

$5.8 \mathrm{pcf}$

5.6

Optimum Water Content
Clayey Sand, CL
15.0 percent $1.0^{*}$
$2.8^{*}$
18.7
Gault Clay, $\mathrm{CH}$
21.0 percent $2.0^{*}$
$5.5^{*}$
26.2
Weald Clay, CH
19.0 percent $3.3^{*}$
$9.1^{*}$
47.9

\section{Multioperator **}

Maximum Dry Density

Sandy Clay, CL $\quad 112.9$ pcf

$1.4 \mathrm{pcf}$

$3.9 \mathrm{pcf}$

3.4

Gault Clay, $\mathrm{CH} \quad 102.3$ pcf

$1.3 \mathrm{pcf}$

$3.6 \mathrm{pcf}$

3.5

Weald Clay, $\mathrm{CH} \quad 106.1$ pcf

$1.7 \mathrm{pcf}$

$4.7 \mathrm{pcf}$

4.4

Optimum Water Content

Sandy Clay, CL $\quad 16.0$ percent $0.8^{*}$

$2.2^{*}$

13.8

Gault Clay, CH

22.0 percent $1.2^{*}$

3. $3^{*}$

15.0

Weald Clay, $\mathrm{CH}$

20.0 percent $1.4^{*}$

$3.9^{*}$

19.5

(Continued) 
Table 9 (Concluded)

\section{Single-Operatort}

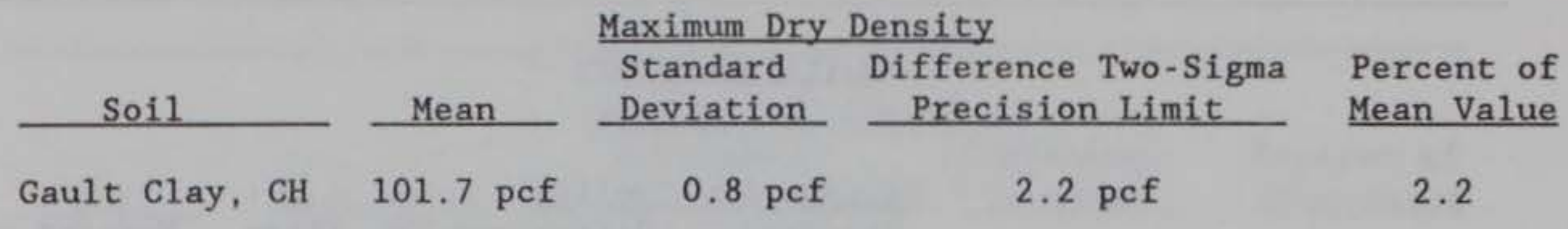

\section{Optimum Water Content}

$\begin{array}{lllll}\text { Gault } \mathrm{Clay}, \mathrm{CH} & 22.0 \text { percent } & 0.2^{*} & 0.6^{*} & 2.7\end{array}$

* Percentage points.

** Multi-operator case are results obtained by 8 operators within RRL.

$\dagger$ Single-operator case represents 8 tests by one operator within RRL. 
Table 10

Test Gradations and Compaction Data Summary (Gordon, Hammon 1 and Miller 1964)

\begin{tabular}{|c|c|c|c|c|c|c|c|}
\hline $\begin{array}{l}\text { Laboratory } \\
\text { Sample } \\
\text { No. } \\
\end{array}$ & $\begin{array}{l}\text { Maximum } \\
\text { Particle } \\
\text { Size } \\
\text { in. } \\
\end{array}$ & $\begin{array}{l}\text { Plus } \\
\text { No. } 4^{*}\end{array}$ & $\begin{array}{l}\text { Minus } \\
\text { No. } 4 \\
\text { Specifio } \\
\text { Gravity } \\
\end{array}$ & $\underline{\mathrm{PI}^{*}}$ & $\underline{L_{L}^{*}}$ & $\begin{array}{c}\text { Miximum } \\
\text { Density } \\
\text { pcf } \\
\end{array}$ & $\begin{array}{l}\text { Opt imum } \\
\text { Wacer } \\
\text { Content" }\end{array}$ \\
\hline $1-4043$ & $\begin{array}{l}\text { No. } 4 \\
3 / 4 \\
1-1 / 2 \\
4\end{array}$ & $\begin{array}{r}0 \\
35 \\
50 \\
62\end{array}$ & 2.84 & 18 & 35 & $\begin{array}{l}126.6 \\
135.9 \\
139.8 \\
144.3\end{array}$ & $\begin{array}{r}12.2 \\
9.1 \\
7.7 \\
7.7\end{array}$ \\
\hline $1-4044$ & $\begin{array}{l}\text { No. } 4 \\
3 / 4 \\
1-1 / 2 \\
4\end{array}$ & $\begin{array}{r}0 \\
24 \\
34 \\
43\end{array}$ & 2.85 & 18 & 36 & $\begin{array}{l}124.3 \\
130.1 \\
133.4 \\
133.8\end{array}$ & $\begin{array}{r}13.0 \\
11.3 \\
9.9 \\
9.5\end{array}$ \\
\hline $1-4045$ & $\begin{array}{l}\text { No. } 4 \\
3 / 4 \\
1-1 / 2 \\
4\end{array}$ & $\begin{array}{r}0 \\
18 \\
26 \\
35\end{array}$ & 2.83 & 10 & 27 & $\begin{array}{l}122.0 \\
128.1 \\
131.0 \\
131.6\end{array}$ & $\begin{array}{r}13.3 \\
11.7 \\
10.6 \\
8.3\end{array}$ \\
\hline $1-4046$ & $\begin{array}{l}\text { No. } 4 \\
3 / 4 \\
1-1 / 2 \\
4\end{array}$ & $\begin{array}{r}0 \\
21 \\
32 \\
42\end{array}$ & 2.85 & 12 & 30 & $\begin{array}{l}123.0 \\
132.9 \\
134.4 \\
132.3\end{array}$ & $\begin{array}{r}13.2 \\
11.1 \\
9.6 \\
9.8\end{array}$ \\
\hline $1-4047$ & $\begin{array}{l}\text { No. } 4 \\
3 / 4 \\
1-1 / 2 \\
4\end{array}$ & $\begin{array}{r}0 \\
25 \\
38 \\
50\end{array}$ & 2.84 & 12 & 35 & $\begin{array}{l}118.7 \\
128.1 \\
133.0 \\
134.3\end{array}$ & $\begin{array}{r}15.0 \\
12.0 \\
10.1 \\
9.6\end{array}$ \\
\hline $1-4047 \mathrm{~A}$ & $\begin{array}{l}\text { No. } 4 \\
3 / 4 \\
1-1 / 2 \\
4\end{array}$ & $\begin{array}{r}0 \\
25 \\
38 \\
50\end{array}$ & 2. 83 & 14 & 34 & $\begin{array}{l}119.4 \\
128.7 \\
133.6 \\
132.8\end{array}$ & $\begin{array}{r}14.6 \\
11.3 \\
9.8 \\
9.9\end{array}$ \\
\hline $1-4048$ & $\begin{array}{l}\text { No. } 4 \\
3 / 4 \\
1-1 / 2 \\
4\end{array}$ & $\begin{array}{r}0 \\
30 \\
44 \\
58\end{array}$ & 2.85 & 15 & 32 & $\begin{array}{l}128.0 \\
135.0 \\
139.8 \\
141.1\end{array}$ & $\begin{array}{r}11.5 \\
9.0 \\
7.9 \\
8.0\end{array}$ \\
\hline $1-4687^{\star *}$ & $\begin{array}{l}\text { No. } 4 \\
3 \\
3 \\
3\end{array}$ & $\begin{array}{l}0 \\
40 \\
50 \\
60\end{array}$ & 2.84 & 16 & 33 & $\begin{array}{l}126.0 \\
136.6 \\
140.1 \\
142.6\end{array}$ & $\begin{array}{r}12.2 \\
9.8 \\
7.5 \\
7.0\end{array}$ \\
\hline $2-890^{\hbar \hbar}$ & $\begin{array}{l}\text { No. } 4 \\
3 \\
3 \\
3\end{array}$ & $\begin{array}{r}0 \\
40 \\
50 \\
60\end{array}$ & 2.80 & 10 & 32 & $\begin{array}{l}118.8 \\
135.2 \\
138.4 \\
140.4\end{array}$ & $\begin{array}{r}14.9 \\
9.4 \\
8.5 \\
7.7\end{array}$ \\
\hline
\end{tabular}

* Percent.

*h Synthetic composite gradations prepared by adding to the soil fraction various percentages of gravel to represent desired grading groups. All other gradations listed above were natural gradations. 
Table 11

Compaction Testing Equipment

(after Gordon, Hammond, and Miller 1964)

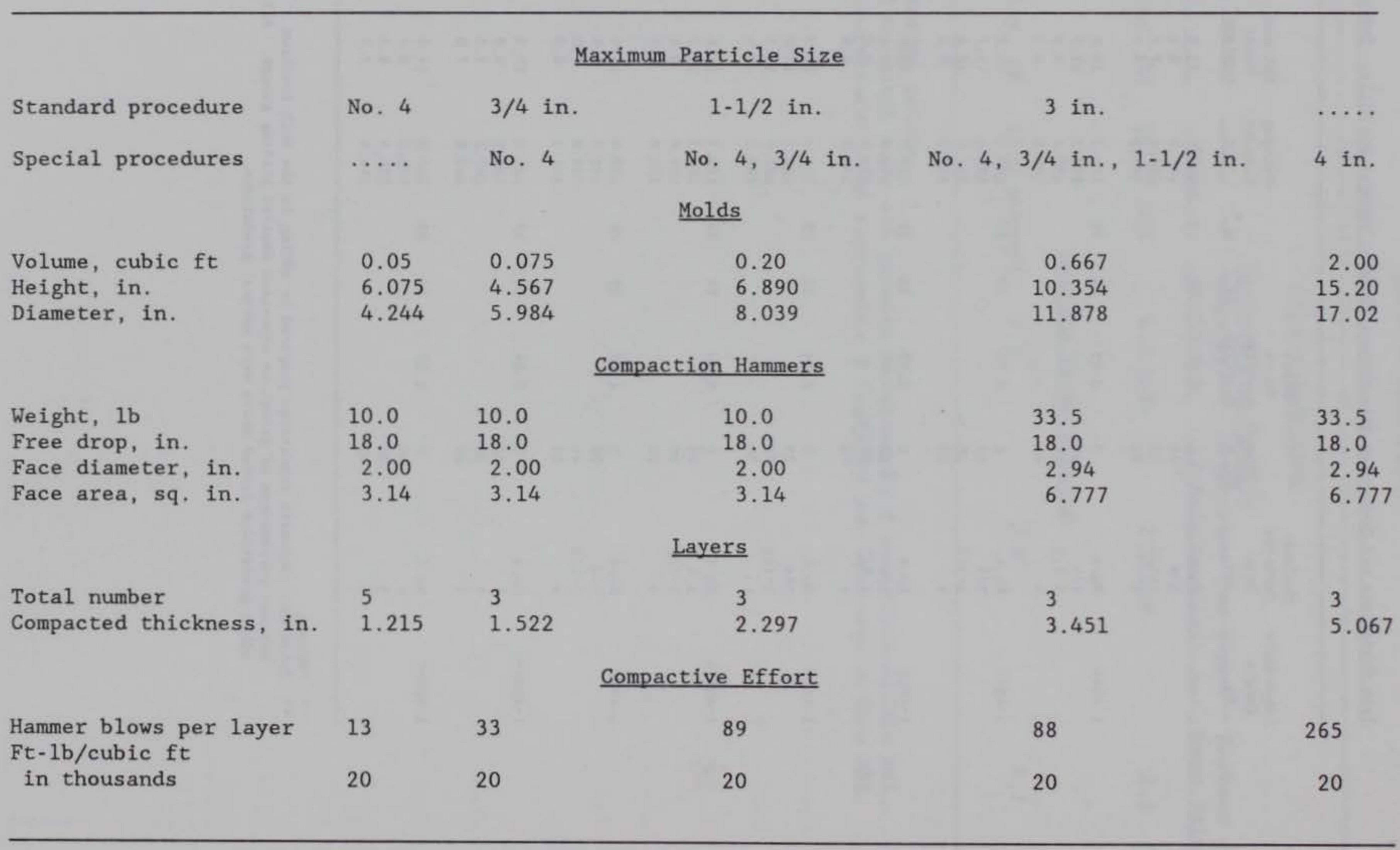


Table 12

Compaction Tests Using Hand-Held Sliding Weight Rammer and

Howard Mechanical Compactor (after Donaghe and Townsend 1975)

\begin{tabular}{|c|c|c|c|c|c|c|c|c|c|}
\hline \multirow[b]{3}{*}{$\begin{array}{l}\text { Mold } \\
\text { Diam } \\
\text { in. }\end{array}$} & \multirow[b]{3}{*}{$\begin{array}{c}\text { Compaction } \\
\text { Effort } \\
\mathrm{ft}-1 \mathrm{~b} / \mathrm{ft}^{3} \\
\end{array}$} & \multicolumn{3}{|c|}{ Rammer* ${ }^{*}$} & & \multirow[b]{3}{*}{$\begin{array}{l}\text { Blows } \\
\text { per } \\
\text { Layer }\end{array}$} & \multirow{3}{*}{$\begin{array}{c}\text { Complete } \\
\text { Coverage } \\
\text { of } \\
\text { Mold Area } \\
\end{array}$} & \multirow{2}{*}{\multicolumn{2}{|c|}{ Blows per Coverage }} \\
\hline & & & Diam of & & & & & & \\
\hline & & $\begin{array}{c}\text { Weight } \\
1 \mathrm{~b} \\
\end{array}$ & $\begin{array}{c}\text { Circular } \\
\text { Face } \\
\text { in. } \\
\end{array}$ & $\begin{array}{l}\text { Drop } \\
\text { in. }\end{array}$ & $\begin{array}{l}\text { No. of } \\
\text { Layers }\end{array}$ & & & $\begin{array}{c}\text { Along } \\
\text { Circumference } \\
\text { of Mold } \\
\end{array}$ & $\begin{array}{c}\text { At } \\
\text { Center of } \\
\text { Specimen } \\
\end{array}$ \\
\hline 6 & 12,420 & 5.5 & 2 & 12 & 3 & 56 & 2 & 24 & 4 \\
\hline 18 & 12,299 & 24.7 & 6 & 24 & 3 & 220 & 7 & $24 * *$ & $6+$ \\
\hline
\end{tabular}

* Compaction equipment: hand-held and mechanical compaction, 6-in. diameter mold;

mechanical compaction, 18-in. diameter mold.

** Plus 6 on last coverage.

$\dagger$ Plus 4 on last coverage. 
Table 13

Test Gradations and Compaction Data Summary

(after Donaghe and Townsend 197j)

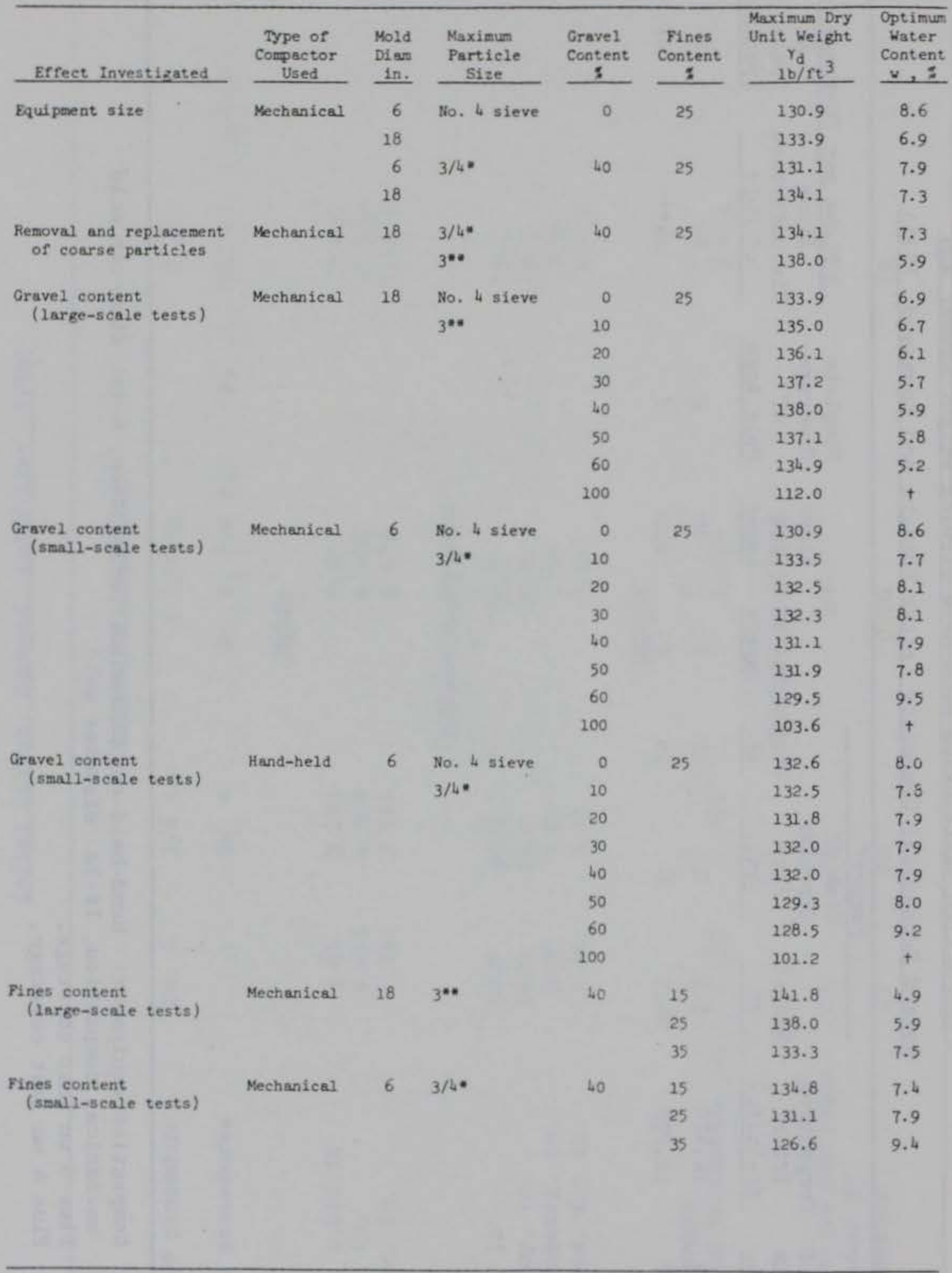

- Sealped and replaced material.

* Full-scale material.

+ Single-point test performed on dry material. 
Table 14

Test Gradations and Compaction Test Data Summary

(after Garga and Madureira 1985)

\begin{tabular}{|c|c|c|c|c|c|c|c|c|c|c|c|c|c|c|}
\hline \multirow{4}{*}{$\begin{array}{l}\text { Test: } \\
\text { series }\end{array}$} & \multirow{4}{*}{$\begin{array}{l}\text { Compaction } \\
\text { energy }\end{array}$} & \multirow{4}{*}{$\begin{array}{l}\text { Mold } \\
\text { di ameter } \\
\text { in. } \\
\end{array}$} & \multirow{4}{*}{$\begin{array}{l}\text { Maximum } \\
\text { particle } \\
\text { size } \\
\end{array}$} & \multirow{4}{*}{$\begin{array}{l}\text { Gravel } \\
\text { content } \\
\text { percent }\end{array}$} & \multicolumn{2}{|c|}{ Iotal Material } & \multicolumn{2}{|c|}{ 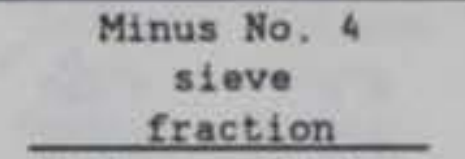 } & \multirow{2}{*}{\multicolumn{2}{|c|}{$\begin{array}{r}\text { Plus No. } 4 \\
\text { sieve fraction } \\
\end{array}$}} & \multicolumn{4}{|c|}{$\begin{array}{l}\text { Characteristics } \\
\text { of minus No. } 4 \\
\text { sieve fraction }\end{array}$} \\
\hline & & & & & \multirow{3}{*}{$\begin{array}{c}\text { Maximum } \\
\text { dry } \\
\text { density } \\
\text { pcf }\end{array}$} & \multirow{3}{*}{$\begin{array}{l}\text { Optimum } \\
\text { water } \\
\text { content } \\
\text { percent }\end{array}$} & \multirow{3}{*}{$\begin{array}{c}\text { Maximum } \\
\text { dry } \\
\text { density } \\
\text { pcf } \\
\end{array}$} & \multirow{3}{*}{$\begin{array}{l}\text { Optimum } \\
\text { water } \\
\text { content } \\
\text { percent }\end{array}$} & & & \multirow{3}{*}{$\begin{array}{c}\text { Liquid } \\
\text { limit } \\
\text { percent }\end{array}$} & \multirow{3}{*}{$\begin{array}{l}\text { Plastic } \\
\text { limit } \\
\text { percent }\end{array}$} & \multirow{3}{*}{$\begin{array}{r}\text { Specific } \\
\text { Bravity } \\
\text { solids } \\
\end{array}$} & \multirow{3}{*}{$\begin{array}{r}\text { Minus } \\
\text { No. } 200 \\
\text { si eve } \\
\text { percent }\end{array}$} \\
\hline & & & & & & & & & $\begin{array}{l}\text { Water } \\
\text { absorption }\end{array}$ & $\begin{array}{l}\text { Bulk } \\
\text { specific }\end{array}$ & & & & \\
\hline & & & & & & & & & condition 8 & gravity. & & & & \\
\hline \multirow[t]{6}{*}{1} & Standard & 4 & No. 4 & 0 & 119.8 & 12.3 & ..... & $\ldots$ & & & 26 & 20 & 2.79 & 41 \\
\hline & & 6 & $3 / 4 \mathrm{in}$. & 30 & 127.3 & 10.2 & 116.1 & 14.0 & Saturated & 2.59 & & & & \\
\hline & & & & 40 & 129.2 & 9.5 & 114.2 & 14.8 & and surface & & & & & \\
\hline & & & & 50 & 130.4 & 8.3 & 109.8 & 15.8 & dried (SSD) & & & & & \\
\hline & & & & 60 & 132.3 & 7.9 & 104.2 & 18.2 & & & & & & \\
\hline & & & & 70 & $131: 7$ & 5.7 & ..... & $\cdots$ & & & & & & \\
\hline \multirow[t]{5}{*}{2} & Standard & 4 & No, 4 & 0 & 117.3 & 14.6 & (n..... & .... & & & 28 & 21 & 2.81 & 51 \\
\hline & & 12 & $1-1 / 2$ in. & 30 & 124.8 & 10.7 & 113.6 & 14.7 & SSD & 2.59 & & & & \\
\hline & & & & 40 & 127.9 & 9.7 & 112,3 & 15.4 & & & & & & \\
\hline & & & & 50 & 130.4 & 8.4 & 110.4 & 16.4 & & & & & & \\
\hline & & & & 60 & 132.3 & 7.3 & 104.2 & 16.7 & & & & & & \\
\hline \multirow[t]{5}{*}{3} & Intermediate & 4 & No. 4 & 0 & 119.8 & 14.1 & $\ldots$ & $\ldots$ & & & 30 & 21 & 2.84 & 46 \\
\hline & & 4 & $3 / 4$ in. & 30 & 131.0 & 9.4 & 121.0 & 13.6 & Dry & 2.59 & & 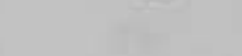 & & \\
\hline & & & & 40 & 131.7 & 8.1 & 116.7 & 14.0 & & & & & & \\
\hline & & & & 50 & 132.9 & 6.3 & 112.3 & 12.3 & & & & & & \\
\hline & & & & 60 & 134.2 & 5.5 & 107.3 & 13.5 & & & & & & \\
\hline \multirow[t]{7}{*}{4} & Intermedi ate & 4 & No. 4 & 0 & 124.8 & 12.6 & (n..... & $\cdots$ & & & 27 & 20 & 2.86 & 45 \\
\hline & & 6 & $3 / 4$ in. & 30 & 130.4 & 9.9 & 124.8 & 14.2 & SSD & 2.59 & & & & \\
\hline & & & & 40 & 131.7 & 9.0 & 117.3 & 14.9 & & & & & & \\
\hline & & & & so & 134.2 & 7.2 & 114.8 & 14.3 & & & & & & \\
\hline & & & & 60 & 136.6 & 6.1 & 111.1 & 15.2 & & & & & & \\
\hline & & & & 65 & 137.3 & 6.6 & 104.8 & 18.0 & & & te & t & & ( \\
\hline & & & & 70 & 136.6 & 5.8 & 101.1 & 18.8 & & & & & & \\
\hline \multirow[t]{5}{*}{5} & Intermediate & 4 & No. 4 & 0 & 121.7 & 14.8 & $\ldots . .$. & $\cdots$ & & & 30 & 21 & 2.84 & 46 \\
\hline & & 6 & $1-1 / 2$ in. & 30 & 131.0 & 9.1 & 120.4 & 13.9 & Dry & 2.59 & & & & \\
\hline & & & & 40 & 134.2 & 8,3 & 119.8 & 13.5 & & & & & & \\
\hline & & & & 50 & 134.8 & 6.6 & 116.1 & 13.3 & & & & & & \\
\hline & & & & 60 & 136.6 & 5.7 & 109.8 & 13.8 & & & & & & \\
\hline \multirow[t]{6}{*}{6} & Intermediate & 4 & No. 4 & 0 & 122.3 & 13.0 & $\ldots$ & $\cdots$ & & & 29 & 20 & 2.82 & 54 \\
\hline & & 12 & $1-1 / 2$ in. & 30 & 129.8 & 9.0 & 119.2 & 12.3 & SSD & 2.59 & & & & \\
\hline & & & & 40 & 132.9 & 7.7 & 118.6 & 12.1 & & & & & & \\
\hline & & & & 50 & 132.9 & 7.0 & 113.6 & 13.0 & & & & & & \\
\hline & & 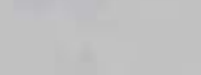 & & 60 & 136.0 & 6.1 & 109.2 & 14.0 & & & & & & \\
\hline & & 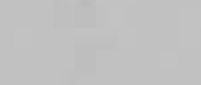 & & 70 & 137.9 & 5.4 & 103.0 & 15.4 & & & & & & \\
\hline
\end{tabular}


Table 14 (Continued)

\begin{tabular}{|c|c|c|c|c|c|c|c|c|c|c|c|c|c|c|}
\hline \multirow{4}{*}{$\begin{array}{l}\text { Test } \\
\text { series }\end{array}$} & \multirow{4}{*}{$\begin{array}{l}\text { Compaction } \\
\text { energy }\end{array}$} & \multirow{4}{*}{$\begin{array}{c}\text { Mold } \\
\text { di ameter } \\
\text { in. } \\
\end{array}$} & \multirow{4}{*}{$\begin{array}{l}\text { Maximum } \\
\text { particle } \\
\text { size } \\
\end{array}$} & \multirow{4}{*}{$\begin{array}{l}\text { Grave 1 } \\
\text { content } \\
\text { percent }\end{array}$} & \multicolumn{2}{|c|}{ Total Material } & \multicolumn{2}{|c|}{$\begin{array}{c}\text { Minus No. } 4 \\
\text { sleve } \\
\text { fraction } \\
\end{array}$} & \multirow{2}{*}{\multicolumn{2}{|c|}{$\begin{array}{r}\text { Plus No. } 4 \\
\text { sieve fraction } \\
\end{array}$}} & \multicolumn{4}{|c|}{$\begin{array}{l}\text { Characteristics } \\
\text { of minus No. } 4 \\
\text { steve fraction }\end{array}$} \\
\hline & & & & & \multirow{3}{*}{$\begin{array}{c}\text { Maximum } \\
\text { dry } \\
\text { density } \\
\text { pcf } \\
\end{array}$} & \multirow{3}{*}{$\begin{array}{l}\text { Optimum } \\
\text { water } \\
\text { content } \\
\text { percent. }\end{array}$} & \multirow{3}{*}{$\begin{array}{c}\text { Maximum } \\
\text { dry } \\
\text { density } \\
\text { pcf } \\
\end{array}$} & \multirow{3}{*}{$\begin{array}{l}\text { Optimum } \\
\text { water } \\
\text { content } \\
\text { percent }\end{array}$} & & & \multirow{3}{*}{$\begin{array}{r}\text { Liquid } \\
\text { limit } \\
\text { percent }\end{array}$} & \multirow{3}{*}{$\begin{array}{l}\text { Plastic } \\
\text { limit } \\
\text { percent }\end{array}$} & \multirow{3}{*}{$\begin{array}{r}\text { Specific } \\
\text { gravity } \\
\text { sollds } \\
\end{array}$} & \multirow{3}{*}{$\begin{array}{c}\text { Minus } \\
\text { No. } 200 \\
\text { sieve } \\
\text { percent }\end{array}$} \\
\hline & & & & & & & & & \multirow{2}{*}{$\begin{array}{l}\begin{array}{l}\text { Water } \\
\text { absorption } \\
\text { condition } \\
\end{array} \\
\end{array}$} & \multirow{2}{*}{$\begin{array}{c}\text { Bulk } \\
\text { specific } \\
\text { gravity }\end{array}$} & & & & \\
\hline & & & & & & & & & & & & & & \\
\hline \multirow[t]{6}{*}{7} & Intermediate & 4 & No. 4 & 0 & 122.9 & 13.5 & -.... & $\ldots$ & & & 30 & 22 & 2.76 & 45 \\
\hline & & 12 & 3 in. & 30 & 126.0 & 10.3 & 114.8 & 13.9 & SSD & 2.59 & & & & \\
\hline & & & & 40 & 130.4 & 8,4 & 116.1 & 13.4 & & & & & & \\
\hline & & & & 50 & 134.2 & 6.8 & 114.2 & 13.0 & & & & & & \\
\hline & & & & 60 & 136.0 & 6.0 & 108.6 & 13.8 & & & & & & \\
\hline & & & & 70 & 137.9 & 5.6 & 103.0 & 16.0 & & & & & & \\
\hline \multirow[t]{6}{*}{8} & Intermediate & 4 & No. 4 & 0 & 122.9 & 12.7 & - & $\cdots$ & & & 28 & 20 & 2.87 & 45 \\
\hline & & 20 & $3 \mathrm{in}$. & 30 & 129.8 & 10.2 & 119.8 & 13.9 & SSD & 2.59 & & & & \\
\hline & & & & 40 & 131.7 & 8.4 & 116.7 & 13.4 & & & & & & \\
\hline & & & & 50 & 133.5 & 7.1 & 114.8 & 14.1 & & & & & & \\
\hline & & & & 60 & 137.3 & 5.6 & 112.3 & 13.3 & & & & & & \\
\hline & & & & 70 & 139.2 & 5.0 & 106.1 & 14.0 & & & & & & \\
\hline \multirow[t]{3}{*}{9} & Modified & 4 & No. 4 & 0 & 126.7 & 12.8 & $\ldots . .$. & -... & & & 30 & 22 & 2.81 & S5 \\
\hline & & 6 & $3 / 4$ in. & 30 & 132.9 & 9.4 & 124.2 & 12.7 & SSD & 2.59 & & & & \\
\hline & & & & 40 & 135,4 & 7.9 & 122.3 & 12.2 & & & & & & \\
\hline 10 & Modified & 4 & No. 4 & 0 & 131.0 & 10.3 & $\ldots$ & $\cdots$ & & & 29 & 19 &..-- & 40 \\
\hline & & 12 & $1-1 / 2$ in. & 30 & 132.9 & 8.8 & 123.5 & 11.3 & SSD & 2.56 & & & & \\
\hline & & & & 40 & 133.5 & 8.4 & 122.3 & 12.4 & & & & & & \\
\hline & & & & 50 & 136.0 & 7.1 & 121.7 & 11.8 & & & & & & \\
\hline & & & & 60 & 136.0 & 7,7 & 115.4 & 14.4 & & & & & & \\
\hline & & & & 65 & 137.3 & 6.6 & 113.6 & 13.7 & & & & & & \\
\hline & & & & 70 & 137.3 & 6.2 & 108.6 & 14.2 & & & & & & \\
\hline 11 & Modified & 4 & No. 4 & 0 & 124.2 & 13.3 & 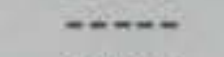 & $\cdots$ & & & 30 & 21 & 2.80 & 55 \\
\hline & & 12 & $1-1 / 2$ in. & 30 & 129.8 & 10.1 & 119.8 & 13.9 & SSD & 2.59 & & & & \\
\hline & & & & 50 & 135.4 & 7.4 & 116.7 & 13.5 & & & & & & \\
\hline 12 & Modified & 4 & No. 4 & 0 & 128.5 & 10.8 & $\ldots$ & $\cdots$ & & & $\cdots$ & $\cdots$ & $\cdots$ & - \\
\hline & & 12 & 3 in. & 40 & 133.5 & 8.8 & 122.3 & 12.8 & SSD & 2.59 & & & & \\
\hline & & & & 50 & 134.8 & 8.3 & 115.4 & 13.0 & & & & & & \\
\hline & & & & 60 & 138.5 & 7.4 & 117.9 & 14.0 & & & & & & \\
\hline & & & & 65 & 139.8 & 6.6 & 117.3 & 13.6 & & & & & & \\
\hline & & & & 70 & 139.2 & 6.2 & 111.1 & 14.2 & & & & & & \\
\hline 13 & Vibratory & 6 & $3 / 4$ in. & 100 & 109.2 & $\cdots$ & 95.5 & 0.0 & Dry & 2.59 & & & & \\
\hline & method & 6 & $1-1 / 2$ in. & 100 & 111.1 & & 99.2 & 0.0 & & & & & & \\
\hline & ASTM-D 2049 & 11 & $1-1 / 2$ in. & 100 & 115.4 & $\cdots$ & 103.0 & 0.0 & & te & & & & \\
\hline & & 11 & 3 in. & 100 & 119.2 & $\ldots$ & 103.6 & 0.0 & & & & & & \\
\hline
\end{tabular}


Table 14 (Concluded)

\begin{tabular}{|c|c|c|c|c|c|c|c|c|c|c|c|c|c|c|}
\hline \multirow{4}{*}{$\begin{array}{l}\text { Test } \\
\text { series }\end{array}$} & \multirow{4}{*}{$\begin{array}{l}\text { Compaction } \\
\text { energy }\end{array}$} & \multirow[b]{3}{*}{$\begin{array}{c}\text { Mold } \\
\text { diameter }\end{array}$} & \multirow{4}{*}{$\begin{array}{l}\text { Maximum } \\
\text { particle } \\
\text { size } \\
\end{array}$} & \multirow[b]{3}{*}{$\begin{array}{l}\text { Gravel } \\
\text { content }\end{array}$} & \multicolumn{2}{|c|}{ Iotal Material } & \multicolumn{2}{|c|}{$\begin{array}{c}\text { Minus No. } 4 \\
\text { sieve } \\
\text { fraction } \\
\end{array}$} & \multirow{2}{*}{\multicolumn{2}{|c|}{$\begin{array}{r}\text { Plus No. } 4 \\
\text { sieve fraction } \\
\end{array}$}} & \multicolumn{4}{|c|}{$\begin{array}{l}\text { Characteristics } \\
\text { of minus No. } 4 \\
\text { sieve fraction }\end{array}$} \\
\hline & & & & & \multirow{3}{*}{$\begin{array}{c}\text { Maximum } \\
\text { dry } \\
\text { density } \\
\text { pcf } \\
\end{array}$} & \multirow{2}{*}{$\begin{array}{l}\text { Optimum } \\
\text { water } \\
\text { content }\end{array}$} & \multirow{2}{*}{$\begin{array}{l}\text { Maximum } \\
\text { dry } \\
\text { density }\end{array}$} & \multirow{2}{*}{$\begin{array}{l}\text { Optimum } \\
\text { water } \\
\text { content }\end{array}$} & & & \multirow{3}{*}{$\begin{array}{l}\text { Liquid } \\
\text { limit } \\
\text { percent }\end{array}$} & \multirow{3}{*}{$\begin{array}{l}\text { Plastic } \\
\text { limit } \\
\text { percent }\end{array}$} & \multirow{3}{*}{$\begin{array}{l}\text { Specific } \\
\text { gravity } \\
\text { solids } \\
\end{array}$} & \multirow{2}{*}{$\begin{array}{r}\text { Minus } \\
\text { No.200 } \\
\text { sieve }\end{array}$} \\
\hline & & & & & & & & & $\begin{array}{l}\text { Water } \\
\text { absorption }\end{array}$ & $\begin{array}{l}\text { Bulk } \\
\text { specific }\end{array}$ & & & & \\
\hline & & in. & & percent & & percent & pef & percent & condition & gravity & & & & percent \\
\hline \multirow[t]{4}{*}{14} & Intermediate & 4 & No. 4 & 0 & 121.7 & 14.1 & $\ldots . .$. & $\cdots$ & & & 29 & 22 & 2.82 & 50 \\
\hline & & 12 & $1-1 / 2$ in. & so & 133.5 & 7.5 & 114.8 & 14.6 & Oven dried & 2.59 & & & & \\
\hline & & & & 50 & 133.5 & 7,8 & 113.6 & 14.5 & Saturated & 2.59 & & & & \\
\hline & & & & $\begin{array}{l}50 \\
50\end{array}$ & $\begin{array}{l}131.7 \\
131.0\end{array}$ & $\begin{array}{l}7.6 \\
8.9\end{array}$ & 111.7 & 15.0 & Oven dried & & & & & \\
\hline \multirow[t]{10}{*}{12} & Intermediate & 4 & No. 4 & 0 & 121.1 & 14.4 & H... & $\ldots$. & Saturated & & 30 & 22 & 2.82 & 55 \\
\hline & & 6 & $3 / 4$ in. & 40 & 130.4 & 9.4 & 114,8 & 15.2 & Dry & 2.59 & & & & \\
\hline & & 12 & $1-1 / 2$ in. & 40 & 130.4 & 8.9 & 116.1 & 14.5 & & & & & & \\
\hline & & 20 & 3 in. & 40 & $130: 4$ & 9.1 & 116.1 & 14.5 & & & & & & \\
\hline & & 6 & $3 / 4$ in. & 50 & 132.3 & B. 2 & 112.3 & 17.5 & & & & & & \\
\hline & & 12 & $1-1 / 2$ in. & 50 & 133.5 & 7.4 & 113.6 & 14.8 & & & & & & \\
\hline & & 20 & $3 \mathrm{in}$. & 50 & 133.5 & 7.6 & 113.6 & 15.2 & & & & & & \\
\hline & & 6 & $3 / 4$ in & 60 & 134.2 & 6.9 & 121.0 & 16.5 & & & & & & \\
\hline & & 12 & $1-1 / 2$ in. & 60 & 134.8 & 6.0 & 121.7 & 15.0 & & & & & & \\
\hline & & 20 & $3 \mathrm{in.}$ & 60 & 136.0 & 5.0 & 123.6 & 14.8 & & & & & & \\
\hline \multirow[t]{7}{*}{16} & Intermediate & 6 & $3 / 4$ in. & 40 & 131.0 & 9.5 & 116.1 & 15.8 & Dry & 2.59 & 30 & 22 & 2.81 & 55 \\
\hline & & 12 & $3 / 4$ in. & 40 & 129.8 & 8.8 & 115.4 & 14.9 & & & & & & \\
\hline & & 12 & $1-1 / 2$ in. & 50 & 132.3 & 7.8 & 111.1 & 15,7 & & & & & & \\
\hline & & 12 & $1-1 / 2$ in. & so & 132.3 & 7.8 & 112.3 & 15.6 & & & & & & \\
\hline & & $\begin{array}{r}6 \\
12\end{array}$ & $3 / 4$ in. & $\begin{array}{l}50 \\
50\end{array}$ & $\begin{array}{l}132.3 \\
130.4\end{array}$ & $\begin{array}{l}8.0 \\
7.8\end{array}$ & $\begin{array}{l}112.3 \\
109.2\end{array}$ & $\begin{array}{l}16.0 \\
15.6\end{array}$ & & & & & & \\
\hline & & 12 & $1-1 / 2$ in. & 60 & 134.2 & 6.3 & 106.7 & $\begin{array}{l}15.6 \\
15.4\end{array}$ & & & & & & \\
\hline & & 20 & $1-1 / 2$ in. & 60 & 132.9 & 6.5 & 105.4 & 15.4 & & & & & & \\
\hline \multirow[t]{4}{*}{17} & Intermediate & e 12 & $1-1 / 2$ in. & 50 & 136.0 & 7.8 & 117.9 & 15.6 & Dry & 2.59 & & & & \\
\hline & & & & 50 & 136.0 & 7.4 & 117.3 & 15.2 & & & & & & \\
\hline & & & & 60 & 135.4 & 5.8 & 109.8 & 14.0 & & & & & & \\
\hline & & & & 60 & 134.8 & 6.3 & 108.6 & 15.8 & & & & & & \\
\hline \multirow[t]{5}{*}{18} & Intermediate & 4 & $3 / 4$ in. & 30 & 129.8 & 10.1 & $\ldots .$. & $\cdots$ & Dry & 2.59 & 31 & 22 & 2.80 & 49 \\
\hline & & 6 & & 30 & 131.0 & 9.6 & -.... & $\ldots$ & & & 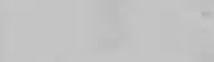 & & & \\
\hline & & 4 & & 40 & 131.7 & 8.1 & -.... & -as & & & te & & & \\
\hline & & 6 & & $\begin{array}{l}40 \\
50\end{array}$ & 132.9 & 8.6 & ....... & $\ldots$ & & & - & 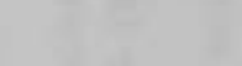 & & \\
\hline & & $\begin{array}{l}4 \\
6\end{array}$ & & $\begin{array}{l}50 \\
50\end{array}$ & $\begin{array}{l}133.5 \\
136.0\end{array}$ & $\begin{array}{l}7.2 \\
7.0\end{array}$ & 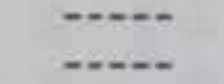 & $\cdots$ & & & & & & \\
\hline 19 & Intermediate & 6 & $1-1 / 2$ in. & 30 & 129.2 & 10.0 & 119.2 & 15.0 & Dry & 2.59 & 29 & 22 & 2.82 & 49 \\
\hline & & 12 & & 30 & 129.2 & 9.8 & 119,2 & 13,8 & & & & & & \\
\hline & & 6 & & 50 & 134.2 & 7.9 & 114.2 & 15.8 & & & & & & \\
\hline & & 12 & & 50 & 134.2 & 7.7 & 113.6 & 15.4 & & & & & & \\
\hline & & 6 & & 70 & 135.4 & 5.8 & 99.8 & 19.2 & & & & & & \\
\hline & & & & 70 & 136.6 & 5.5 & 99.8 & 19.4 & & & & & & \\
\hline
\end{tabular}




\begin{tabular}{|c|c|}
\hline Gradation No. & Description \\
\hline 1 & $\begin{array}{l}\text { MTwUS 3-IN. FULL-SCALE MATERIAL, } 20 \text { percent plus } 3 / 4 \text {-in., } 28 \text { percent gravel, } \\
28 \text { percent minus No. } 200\end{array}$ \\
\hline $1 \mathrm{~A}$ & $\begin{array}{l}\text { MINUS 2-IH. FRACrION, } 12 \text { percent plus } 3 / 4-1 \text { n., } 20.9 \text { percent gravel, } \\
30.8 \text { percent minus No. } 200\end{array}$ \\
\hline 1B & MINUS 3/4-IW. FRACTION, 10 percent grave1, 35 percent minus No. 200 \\
\hline IC & MINUS HO. 4 FRACTION, 38.9 percent minus No. 200 \\
\hline 2 & $\begin{array}{l}\text { MINUS } 3 \text {-IN. FILL.-SCALE MATERTAL, } 40 \text { percent plus } 3 / 4-1 \mathrm{n},, 46 \text { percent gravel, } \\
21 \text { percent minus No. } 200\end{array}$ \\
\hline $2 \mathrm{~A}$ & $\begin{array}{l}\text { MINUS 2-IN. FRACTION, } 23,6 \text { percent plus } 3 / 4-1 \text { n., } 31.2 \text { percent gravel, } \\
26.8 \text { percent minus No. } 200\end{array}$ \\
\hline $1 \mathrm{~B}$ & MINUS 3/4-IN. FRACTION identical to that of Gradation No, 1 \\
\hline $1 \mathrm{C}$ & HINUS NO. 4 FRACTION identical to that of Gradation No. 1 \\
\hline
\end{tabular}

MINUS 3-IN. FULL-SCAL.E mATERTAL, 20 percent plus $3 / 4-$ in., 52 percent gravel, 28 percent minus No. 200

3A MINUS 2-IN. FRACTION, 18.4 percent plus 3/4-in., 51 percent gravel, 28.6 percent minus No. 200

MINUS 3/4-IR. FRACTION, 40 percent grave1, 35 percent minus No. 200

MINUS No. A FRACTION, 58.3 percent minus No. 200

MINUS 3-IN. FUL.L-SCAI.E MATERIAL, 40 percent plus $3 / 4$-in., 64 percent gravel, 21 percent minus No. 200

MINUS 2-IN. FRACTION, 35.8 percent plus $3 / 4-1$ n., 61.5 percent gravel, 22,5 percent minus No, 200 


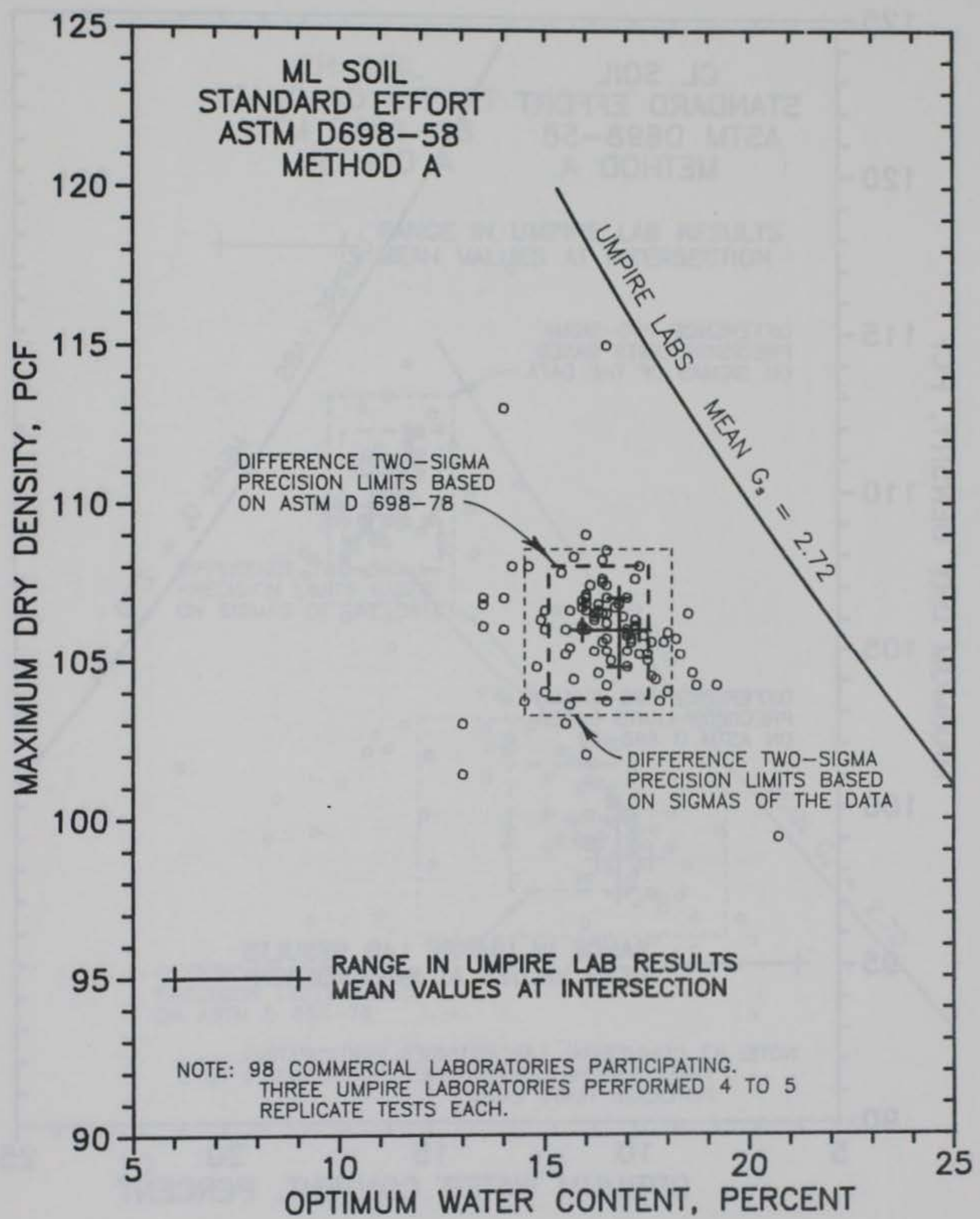

Figure 1. $1964 \mathrm{ACIL}$ study, results of standard effort compaction tests by commercial and umpire laboratories on "standard" ML soil 


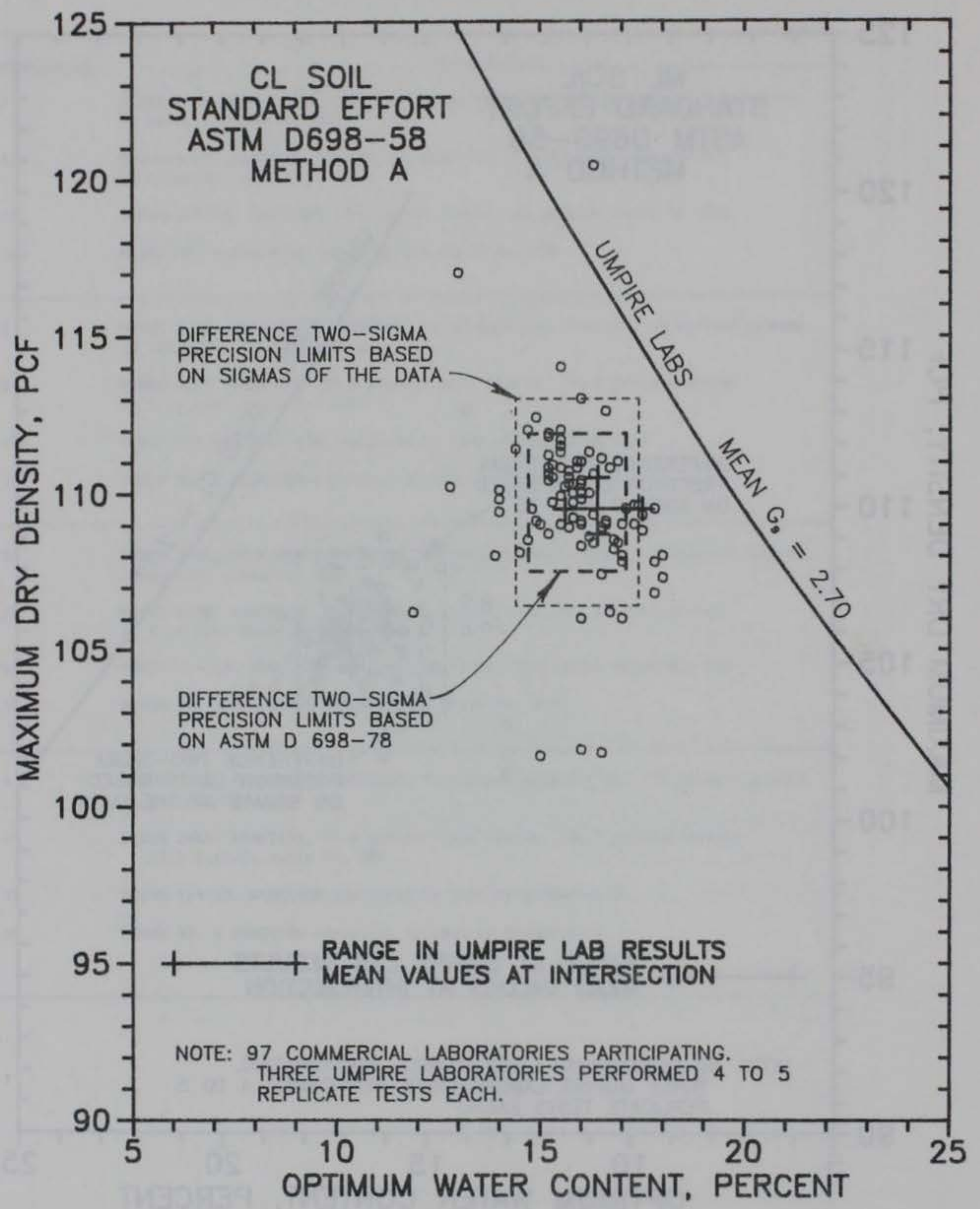

Figure 2. 1964 ACIL study, results of standard effort compaction tests by cominercial and umpire laboratories on "standard" CL soil 


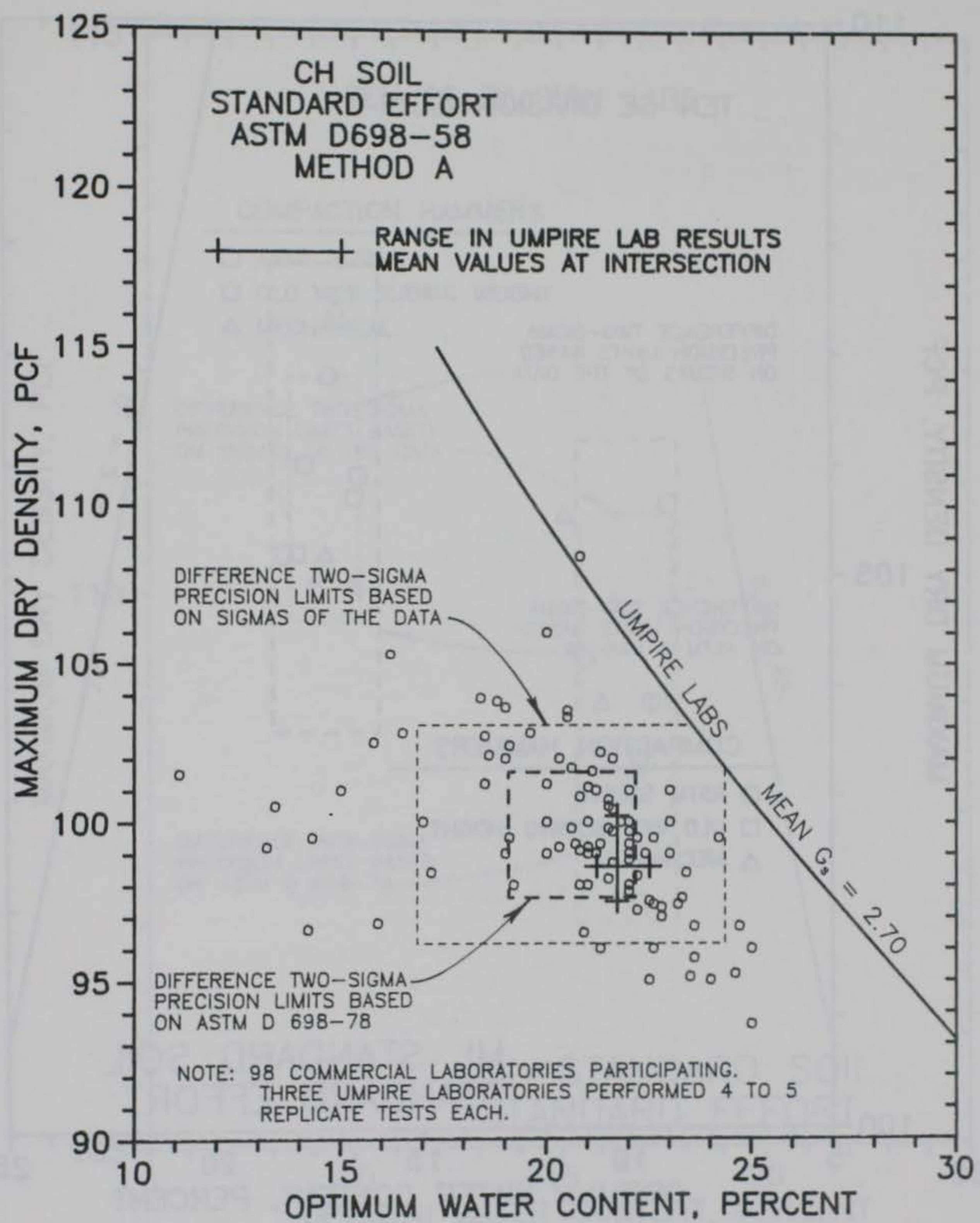

Figure 3. $1964 \mathrm{ACIL}$ study, results of standard effort compaction tests by commercial and umpire laboratories on "standard" $\mathrm{CH}$ soil 


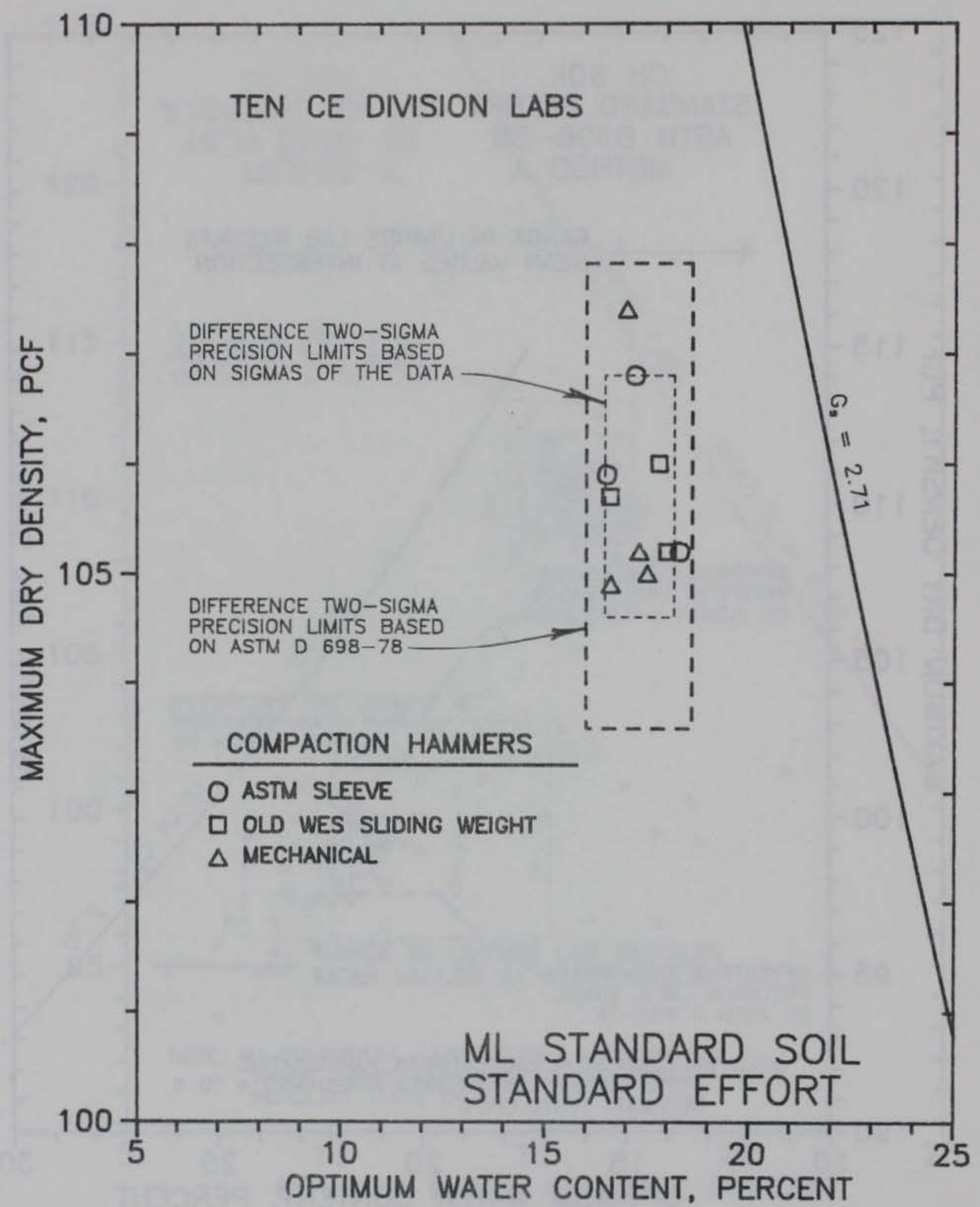

Figure 4. Results of standard effort compaction tests by $10 \mathrm{CE}$ Division Laboratories on the ACIL "standard" ML soil 


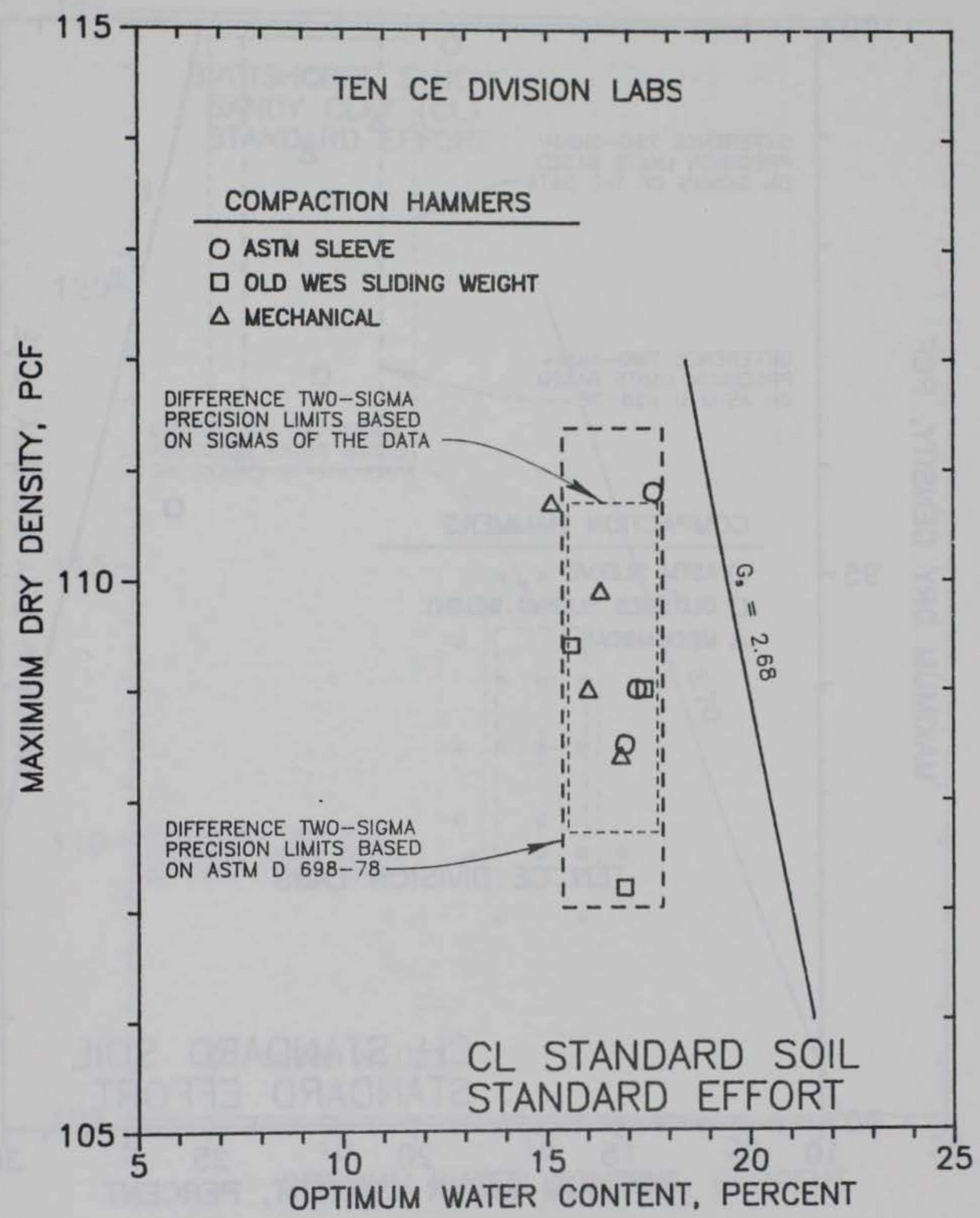

Figure 5. Results of standard effort compaction tests by $10 \mathrm{CE}$ Division Laboratories on the ACIL "standard" CL soil 


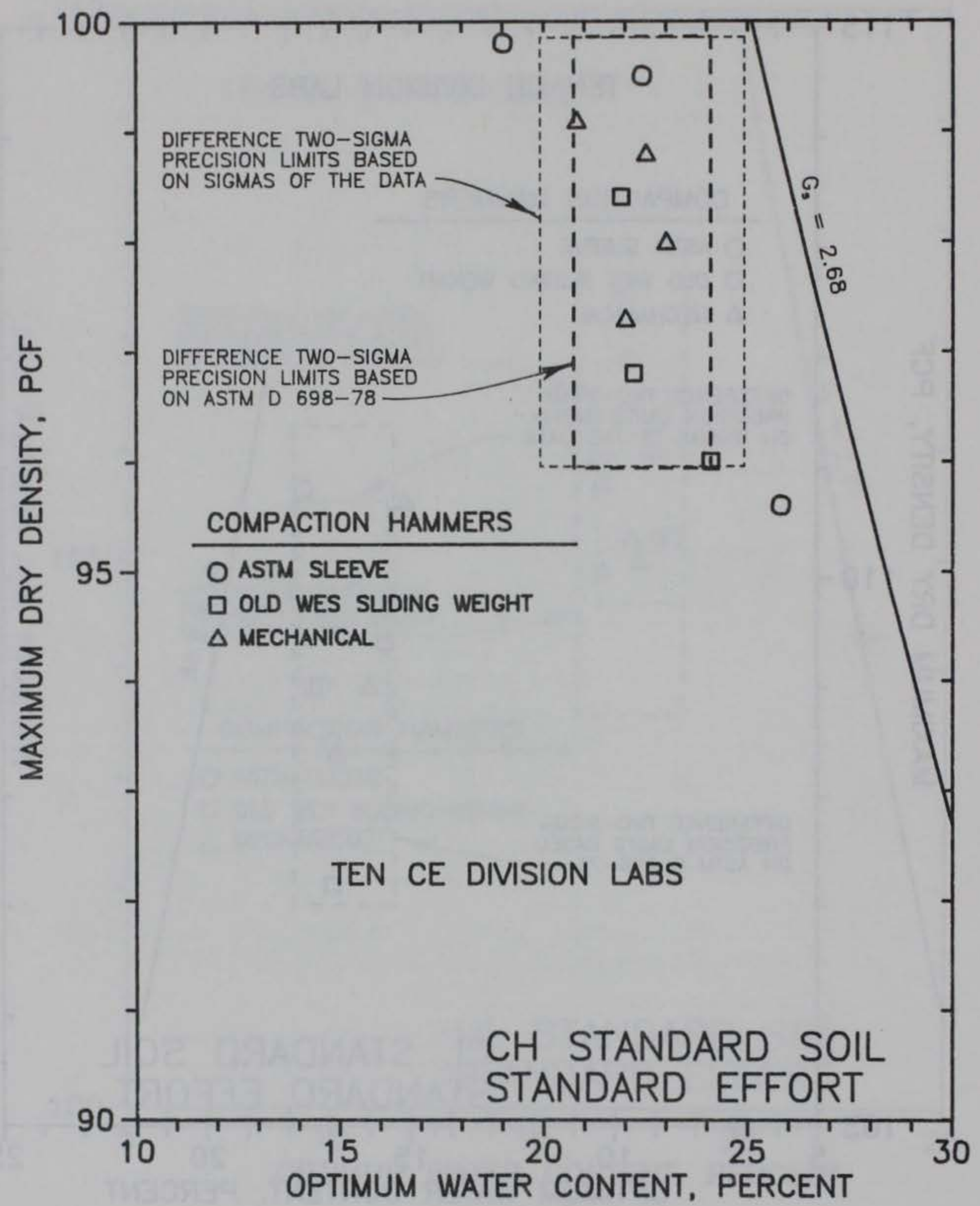

Figure 6. Results of standard effort compaction tests by $10 \mathrm{CE}$ Division Laboratories on the ACIL "standard" $\mathrm{CH}$ soil 


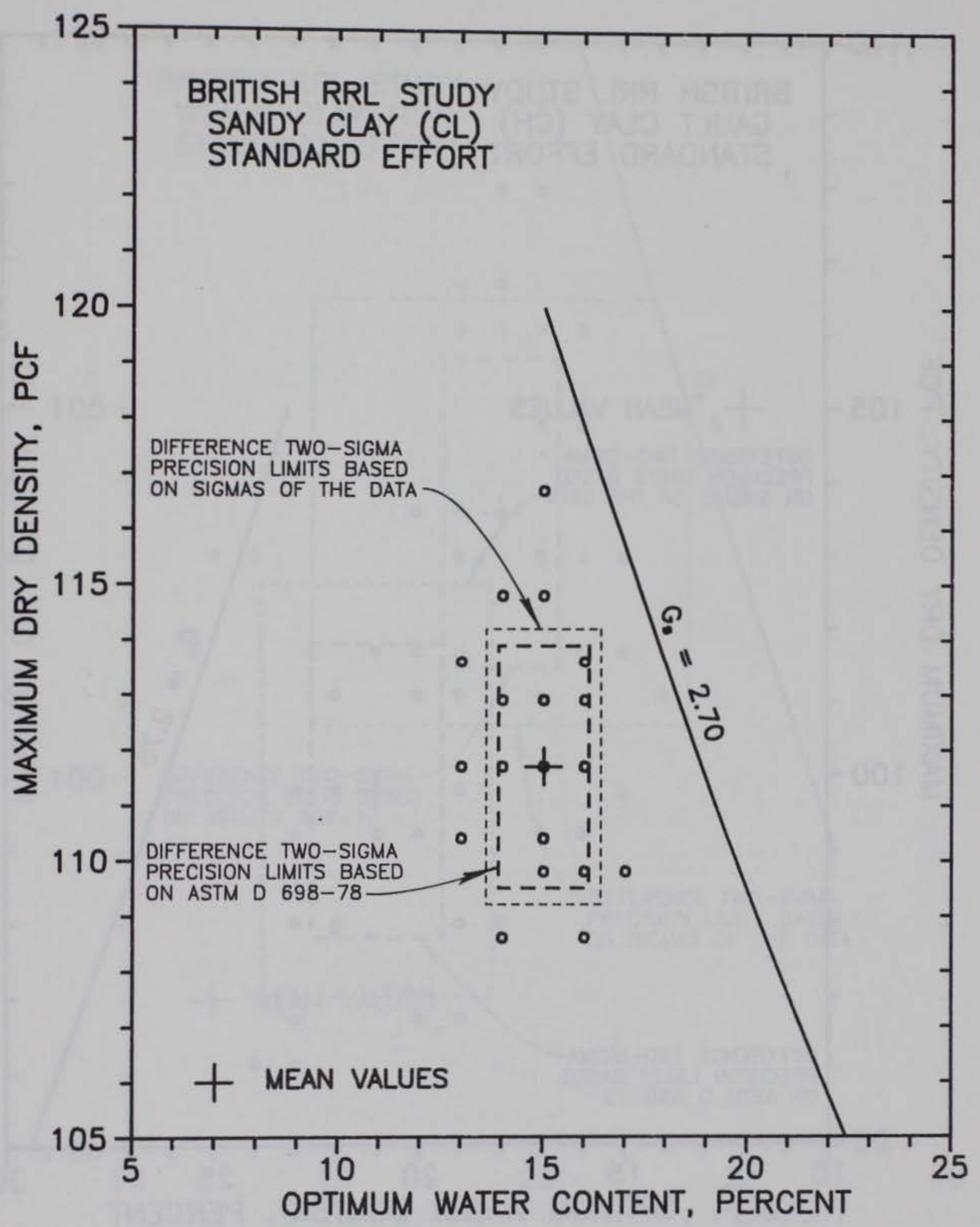

Figure 7. 1970 British RRL study, results of standard effort compaction tests by various government, university, and private laboratories on a sandy clay (CL) 


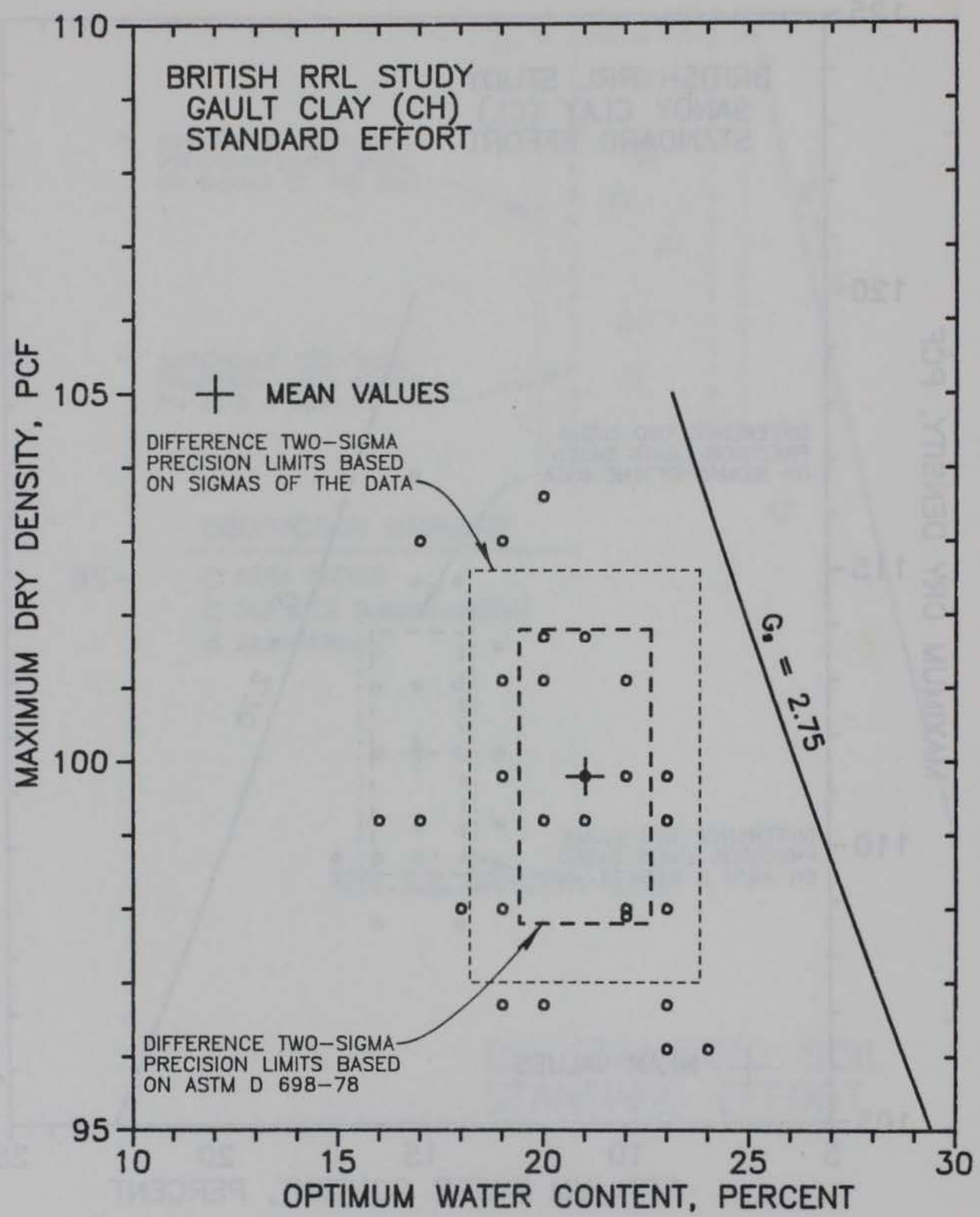

Figure 8. 1970 British RRL study, results of standard effort compaction tests by various government, university, and private laboratories on Gault clay $(\mathrm{CH})$ 


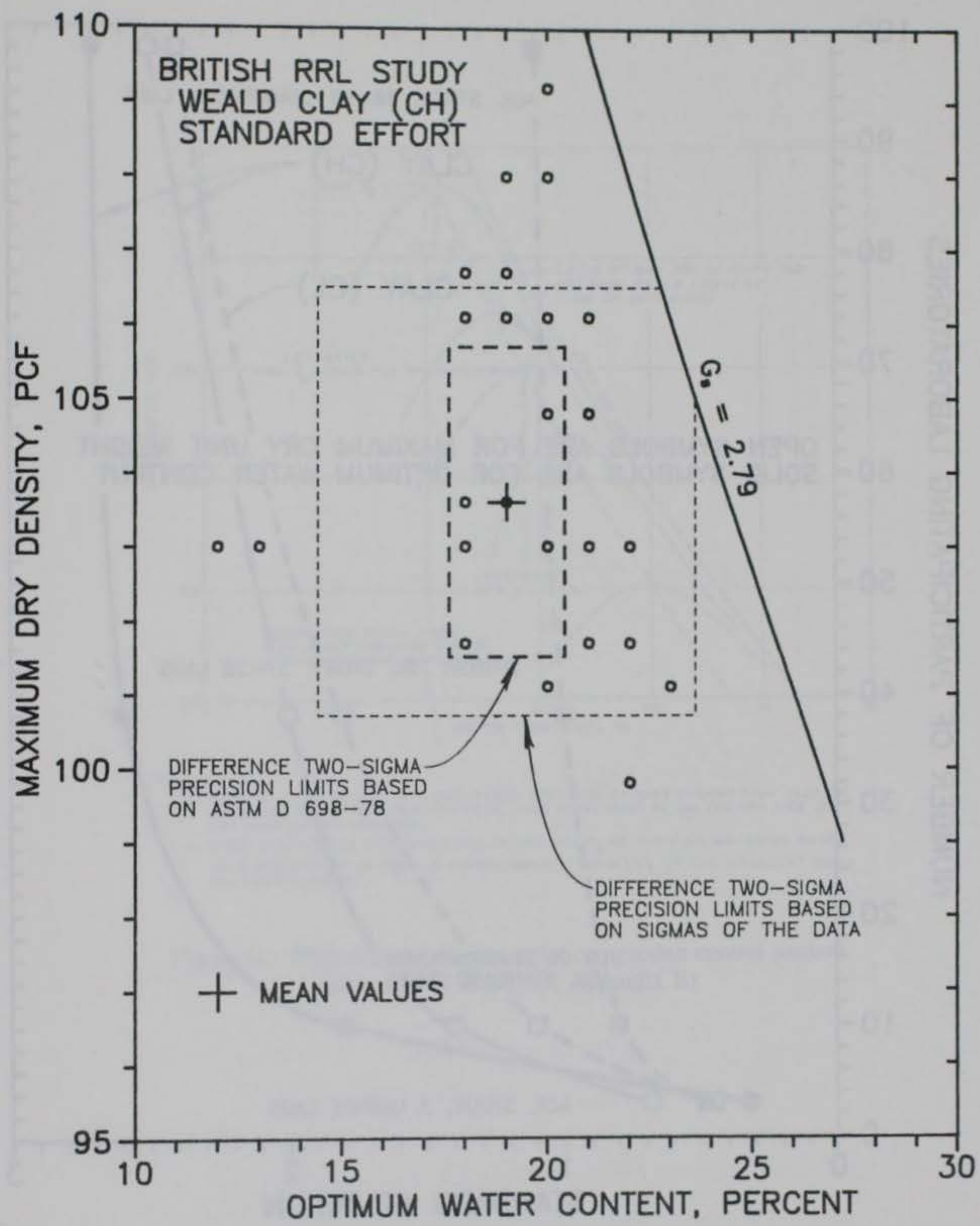

Figure 9. 1970 British RRL study, results of standard effort compaction tests by various government, university, and private laboratories on Weald clay $(\mathrm{CH})$ 


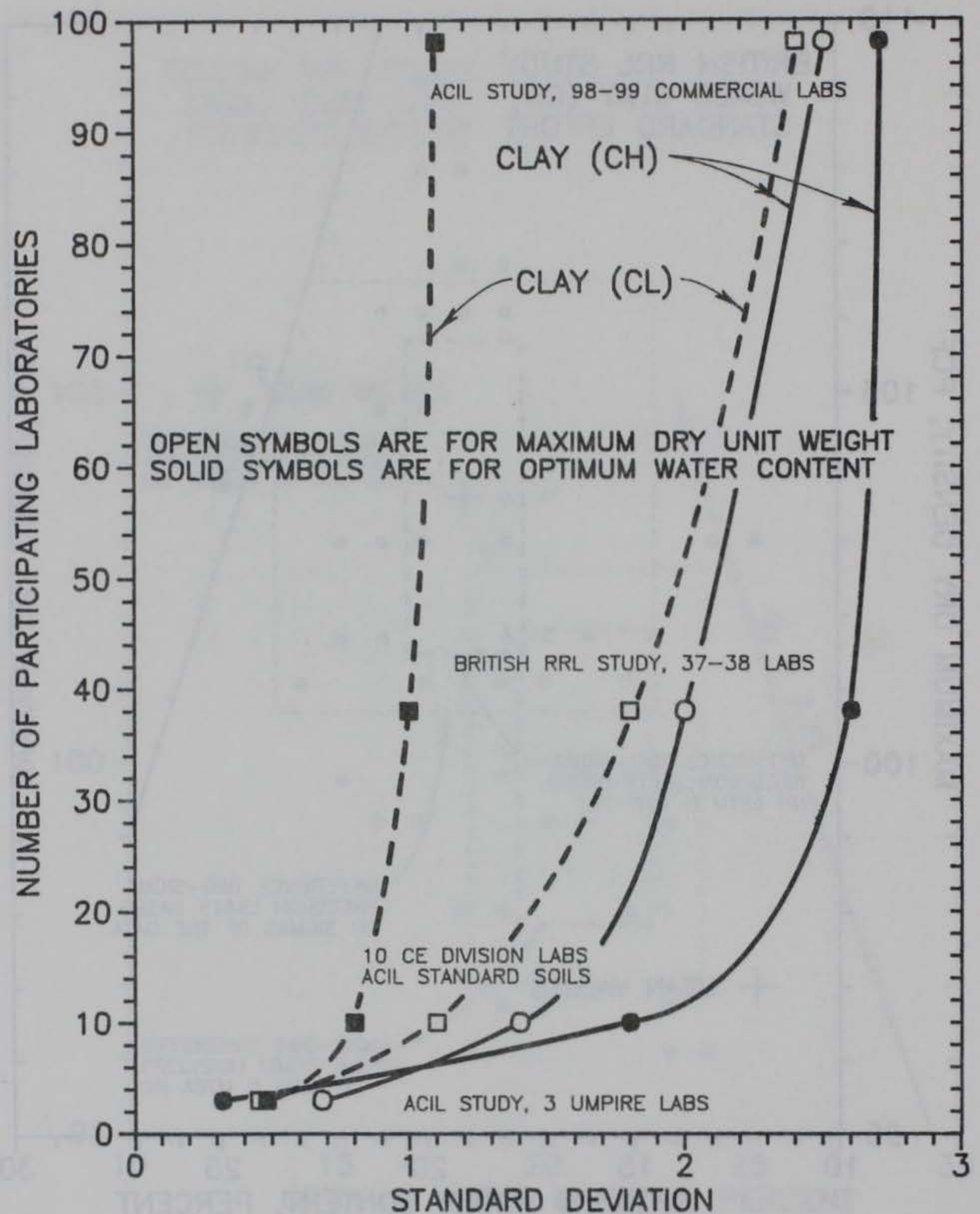

Figure 10. Variations in standard deviations of multilaboratory standard effort compaction parameters for clay soils of the ACIL and British RRL studies with number of participating laboratories 


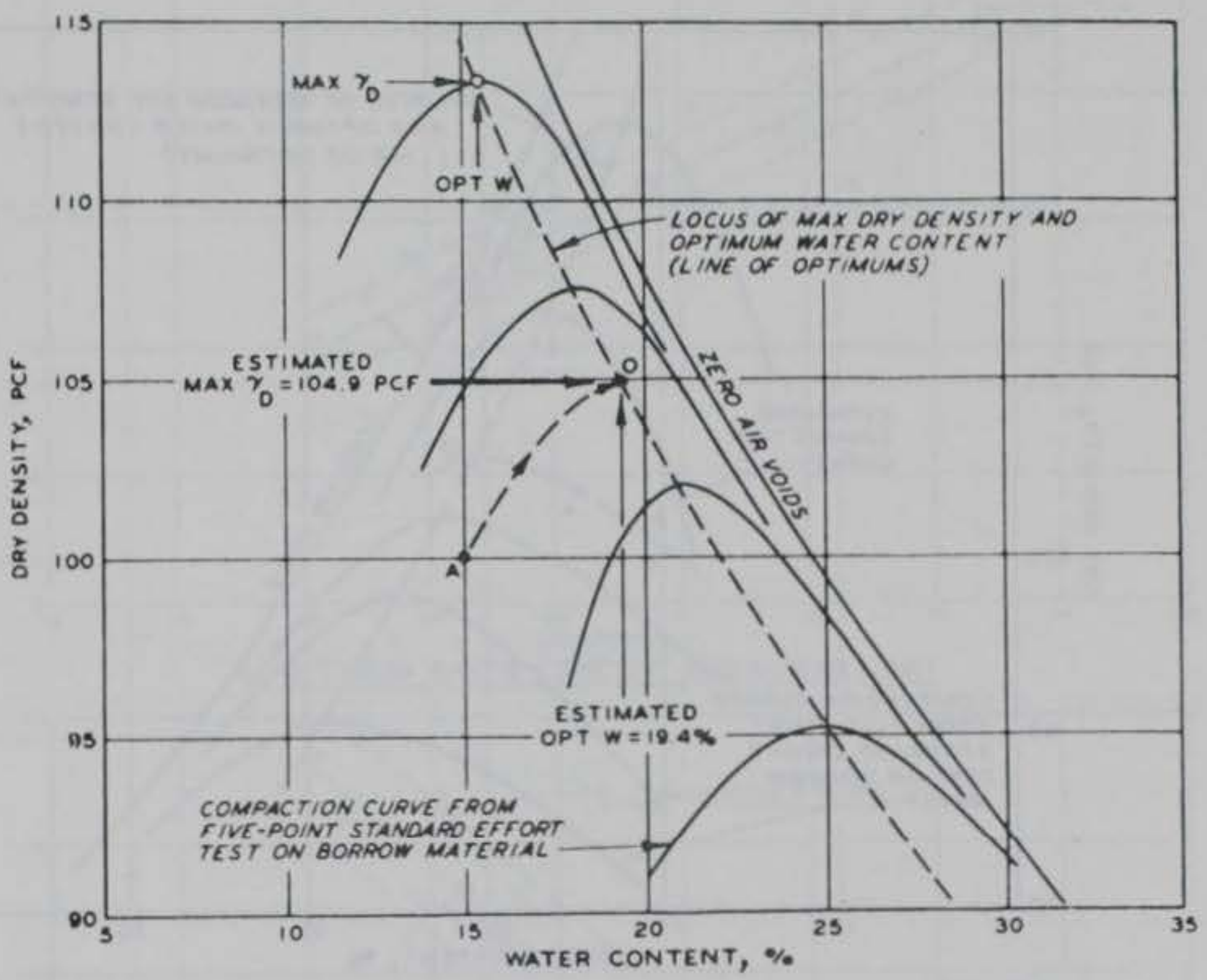

PROCEDURE

1. POINT A IS THE RESULT OF A ONE-POINT STANDARD EFFORT COMPACTION TEST ON MATERIAL FROM FIELD DENSITY TEST. THIS POINT MUST BE ON THE DRY SIDE OF OPTIMUM WATER CONTENT.

2. POINT O GIVES THE ESTIMATED OPT W AND MAX $\gamma_{0}$ OF THE FILL MATERIAL BASED ON A PROJECTION OF POINT A APPROXIMATELY PARALLEL TO THE ADJACENT COMPACTION CURVES.

Figure 11. Illustration of the one-point compaction control method (after EM 1110-2-1911, Appendix B) 


$$
=
$$



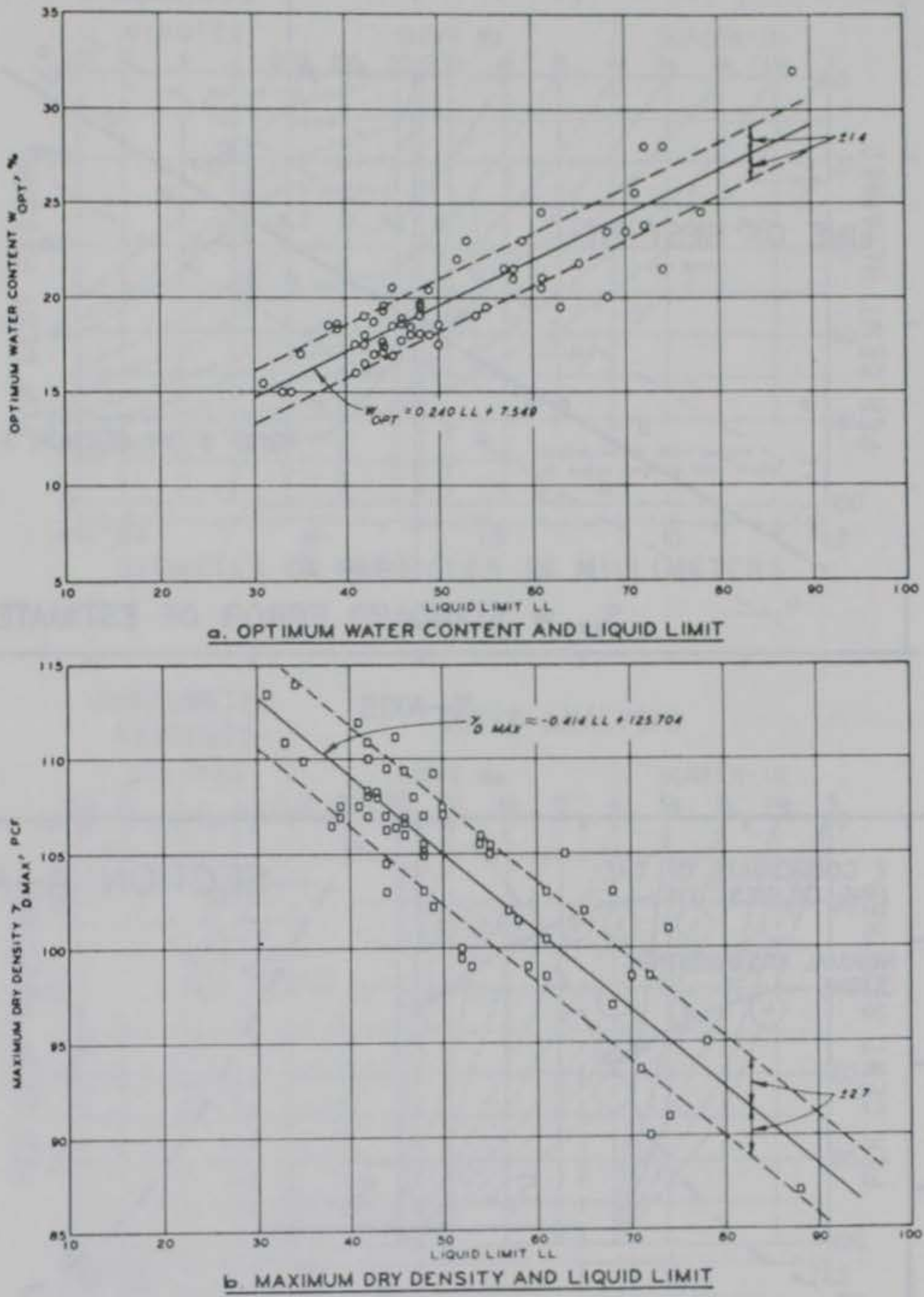

Figure 13. Examples of correlations among maximum dry density, optimum water content and Liquid Limit (after EM 1110-2-1911, Appendix B) 

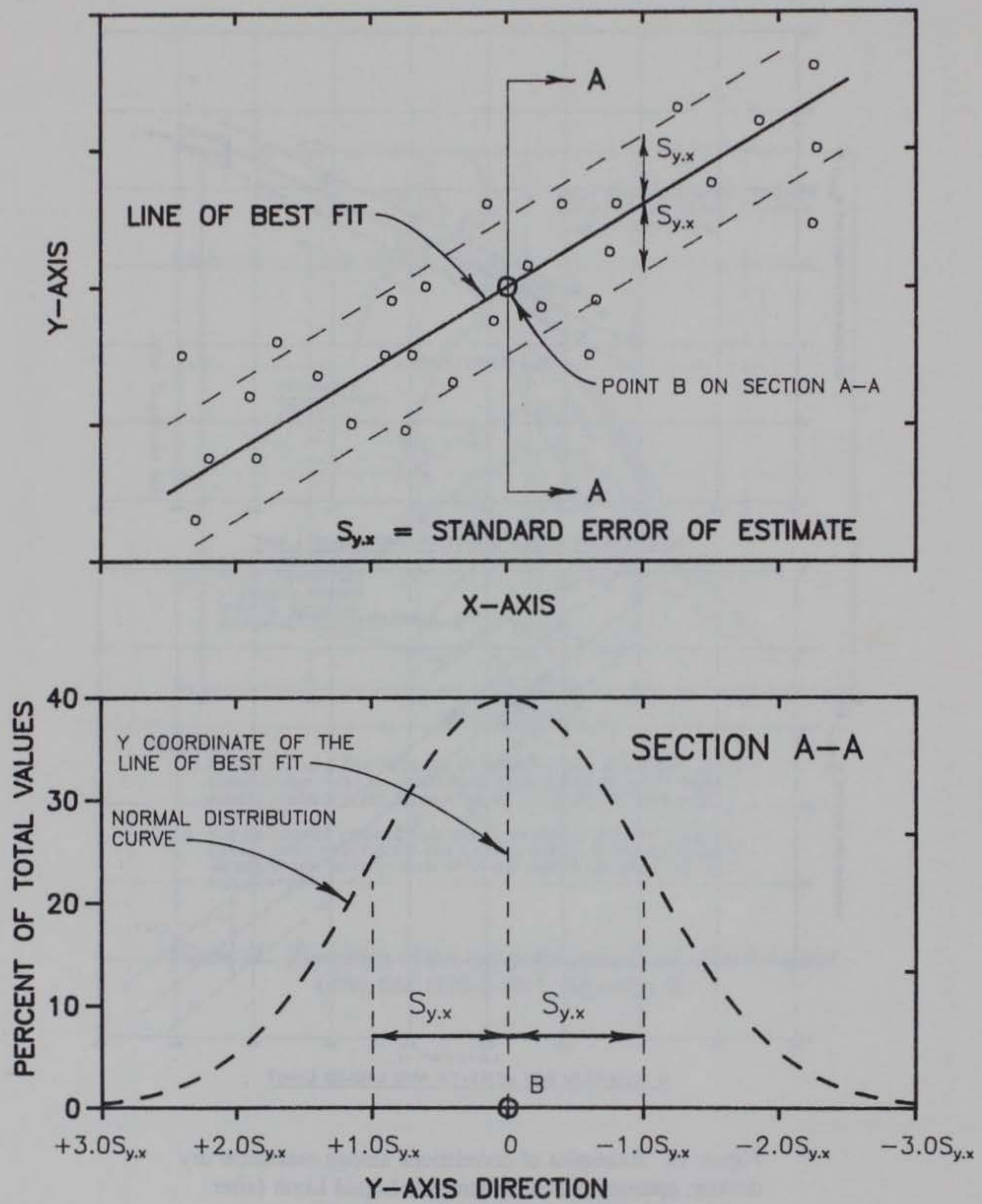

Figure 14. Concept of standard error of the estimate for a linear regression analysis 


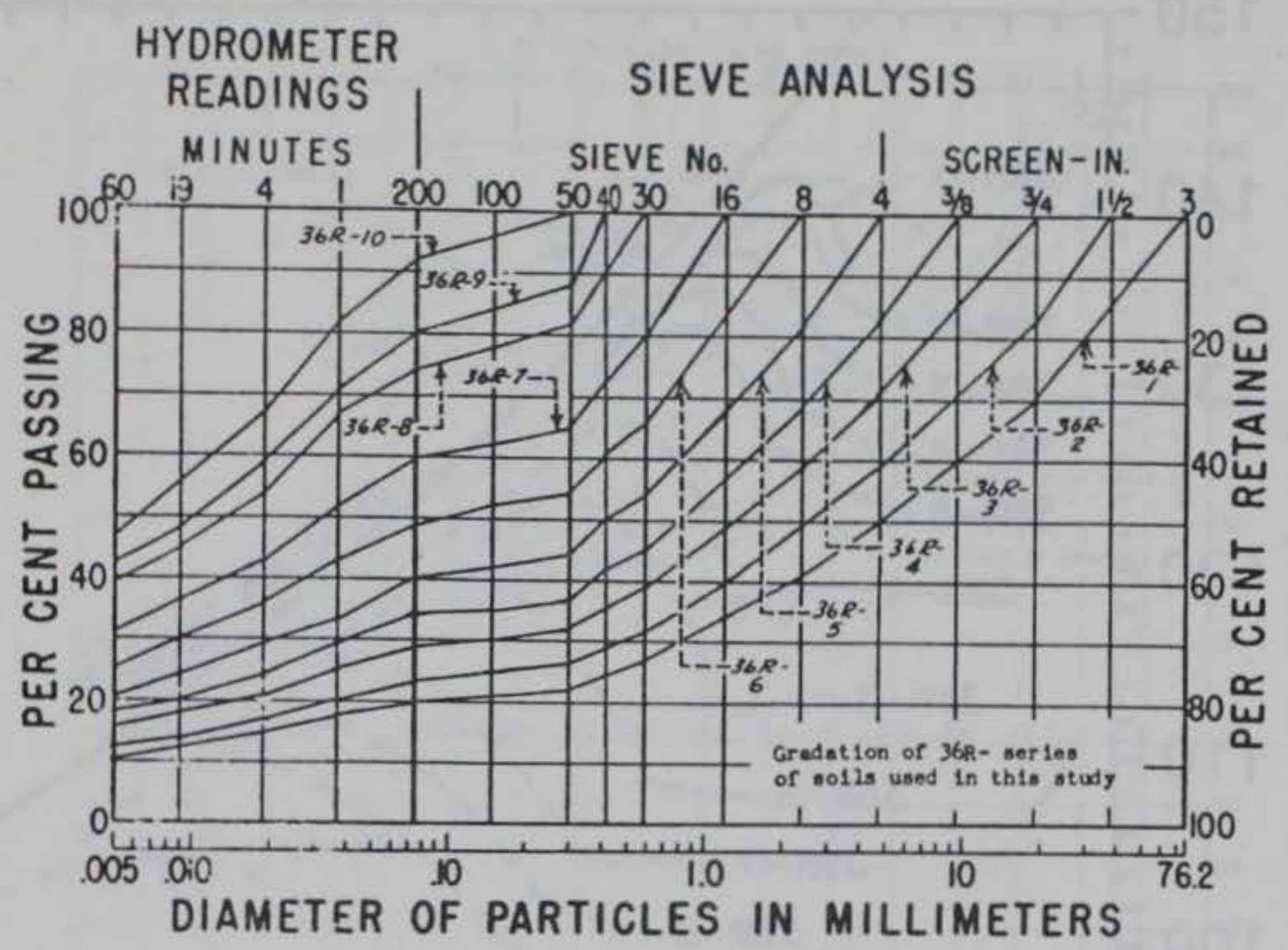

A

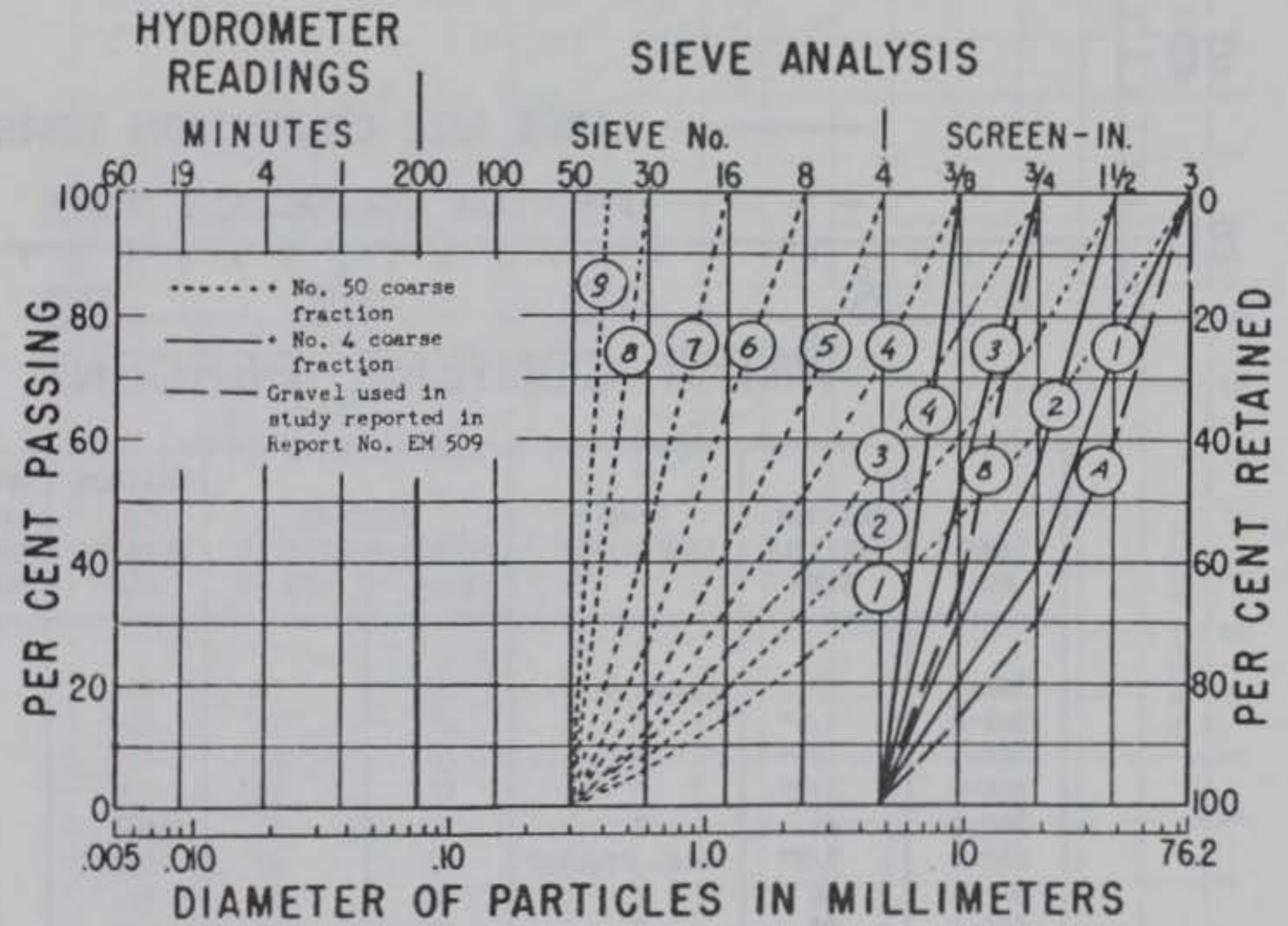

Figure 15. Grain-size distribution curves for materials employed in the testing program (after USBR 1963) 


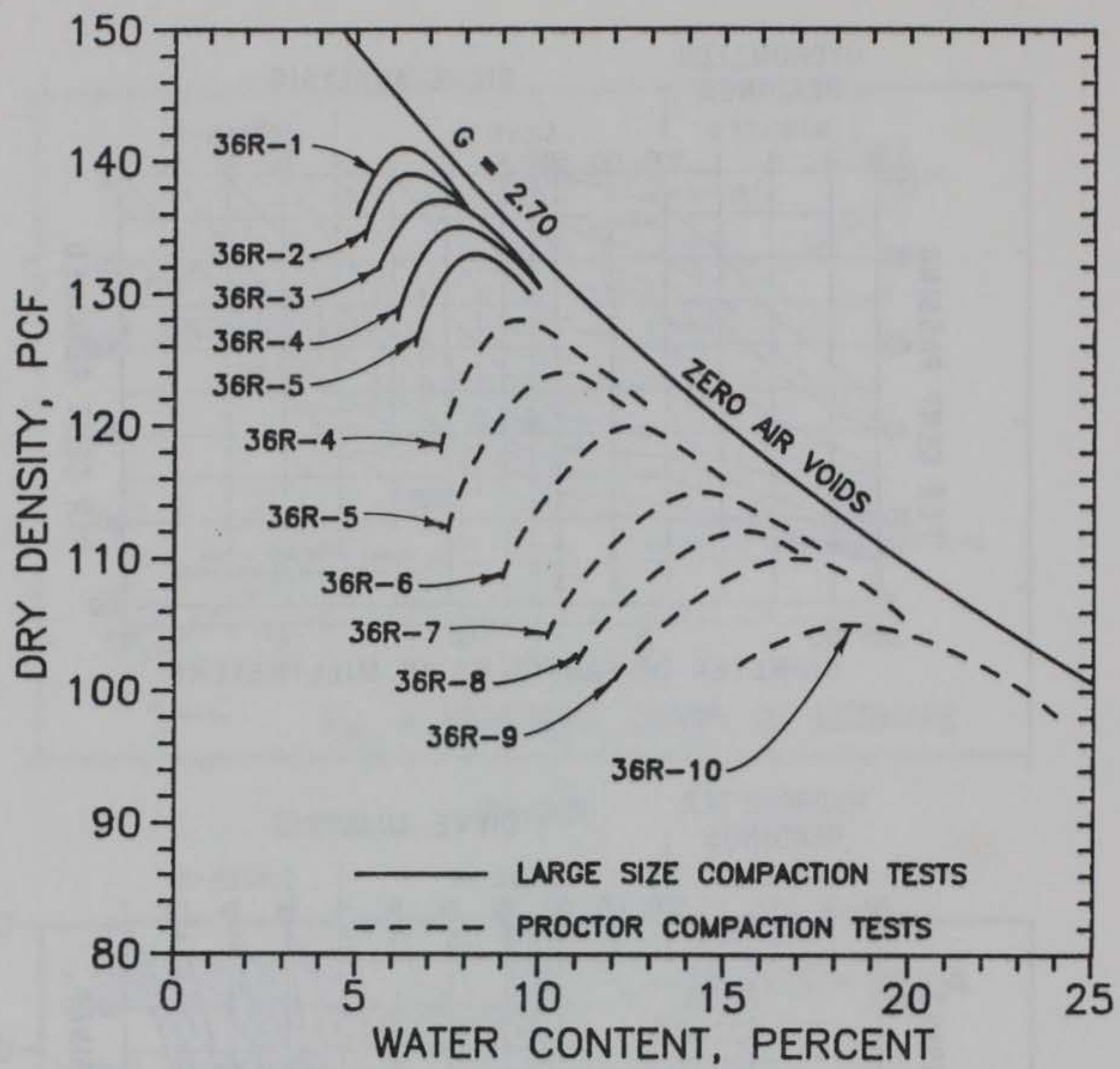

\begin{tabular}{|c|c|c|c|c|c|c|}
\hline $\begin{array}{l}\text { SNUPLE } \\
\text { MO. }\end{array}$ & $\begin{array}{l}\text { MAXIVUM } \\
\text { PARTICLE } \\
\text { SIZE }\end{array}$ & $\begin{array}{c}\text { TYPE } \\
\text { COMPACTION }\end{array}$ & $\begin{array}{r}\text { P } \\
\text { COARSE } \\
+ \text { +MO. } 4\end{array}$ & $\begin{array}{l}\text { EET } \\
\text { URTICLES } \\
+ \text { +WO. } 50\end{array}$ & $\begin{array}{l}\text { MXIIMYN } \\
\text { DRY } \\
\text { DENSITY } \\
\text { PCF }\end{array}$ & $\begin{array}{l}\text { OPTIMUM } \\
\text { MATER } \\
\text { CONTENT } \\
\text { PERCENT }\end{array}$ \\
\hline $36 R-1$ & 3" & Large Size & 50 & 78 & 141 & 6.2 \\
\hline $36 R-2$ & $1.5^{\prime \prime}$ & " & 41 & 74 & 139 & 6.3 \\
\hline $36 R-3$ & $3 / 4^{\prime \prime}$ & " & 29 & 68 & 137 & 7.0 \\
\hline $36 R-\angle$ & $3 / 8^{\prime \prime}$ & $"$ & 17 & 63 & 135 & 7.6 \\
\hline $36 R-5$ & $\# 4$ & " & 0 & 55 & 133 & 8.2 \\
\hline $36 R-4$ & $3 / 8^{\prime \prime}$ & Std. Proctor & 17 & 63 & 128 & 9.4 \\
\hline $36 R-5$ & $\#$ & u & 0 & 55 & 124 & 10.7 \\
\hline $36 R-6$ & $\# 8$ & " & 0 & 46 & 120 & 12.6 \\
\hline $36 R-7$ & \#16 & " & 0 & 35 & 115 & 14.4 \\
\hline $36 R-8$ & $\# 30$ & $"$ & 0 & 18 & 112 & 15.8 \\
\hline $36 R-9$ & $\# 40$ & $"$ & 0 & 12 & 110 & 17.1 \\
\hline $36 R-10$ & $\# 50$ & $"$ & 0 & 0 & 105 & 18.2 \\
\hline
\end{tabular}

Figure 16. Compaction curves for tests performed to determine effects of large particles (after USBR 1963) 

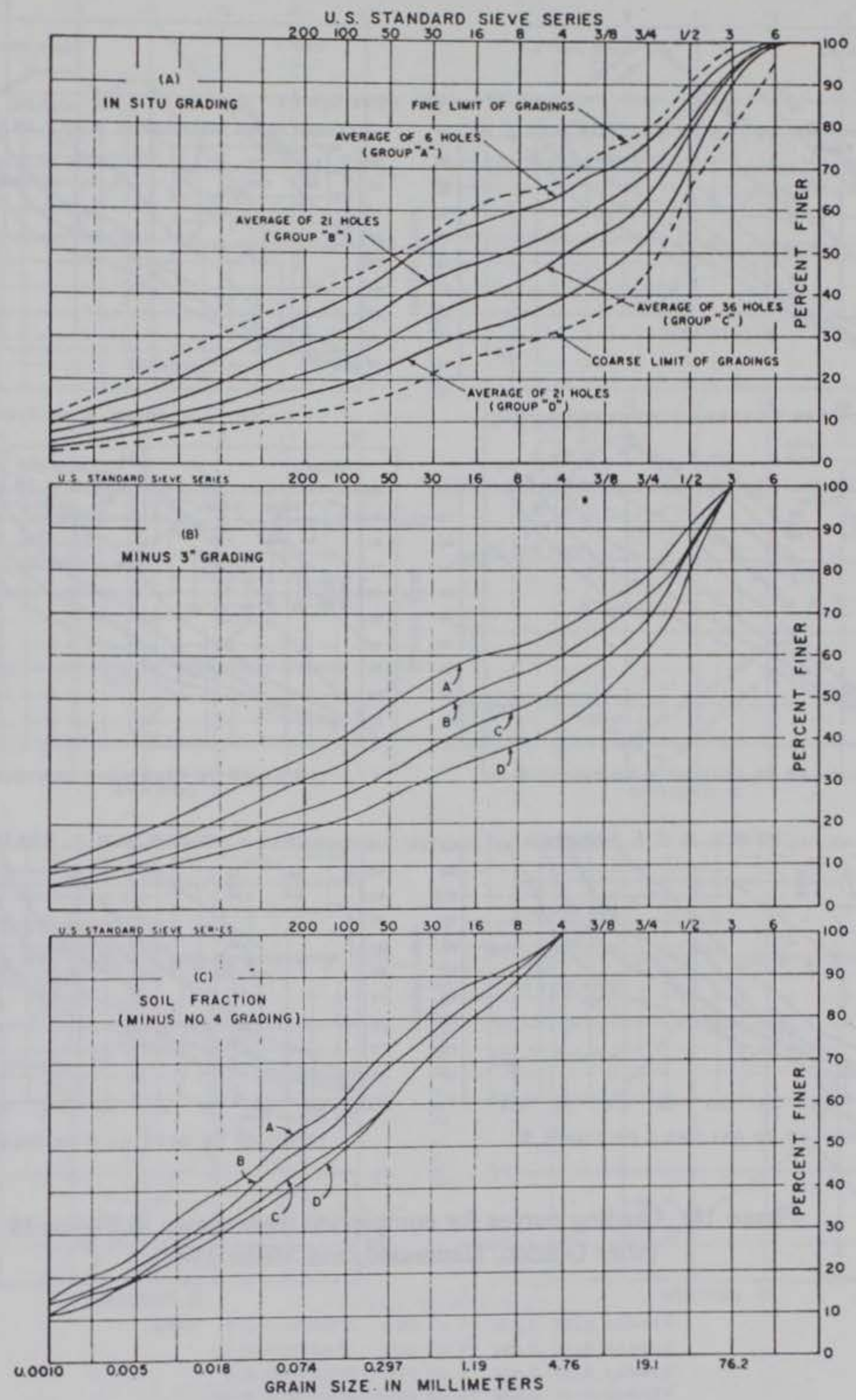

Figure 17. Grading curves representing range of materials (after Gordon, Hammond, and Miller 1964) 
US STANDARD SIEVE SERIES

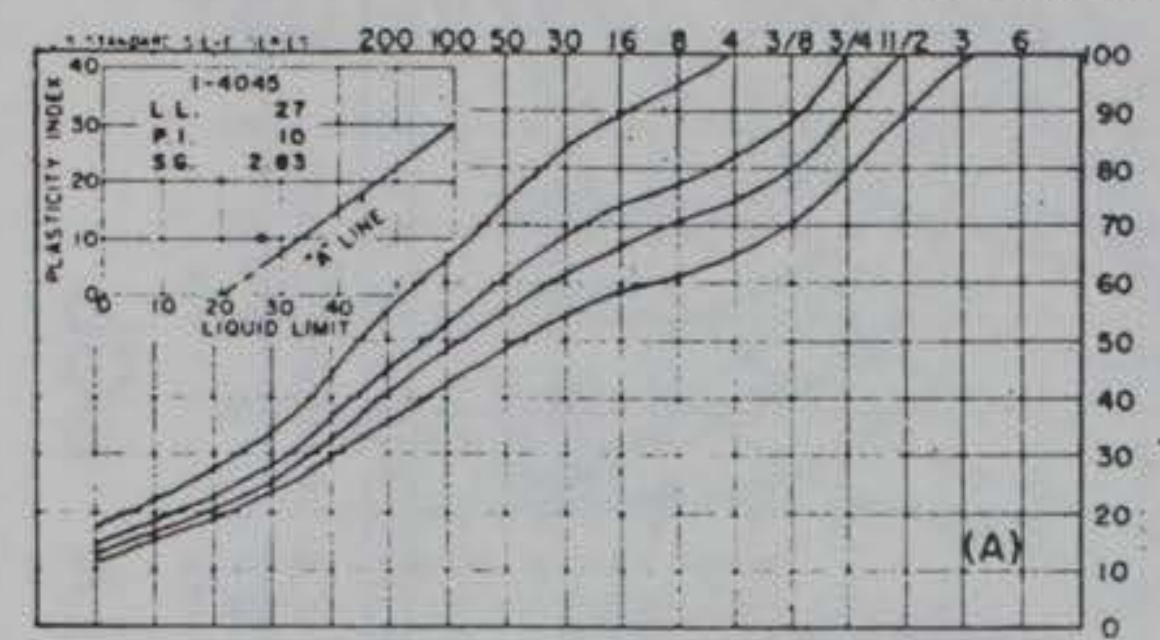

SIMILAR TO GRADING A ON FIGURE 8

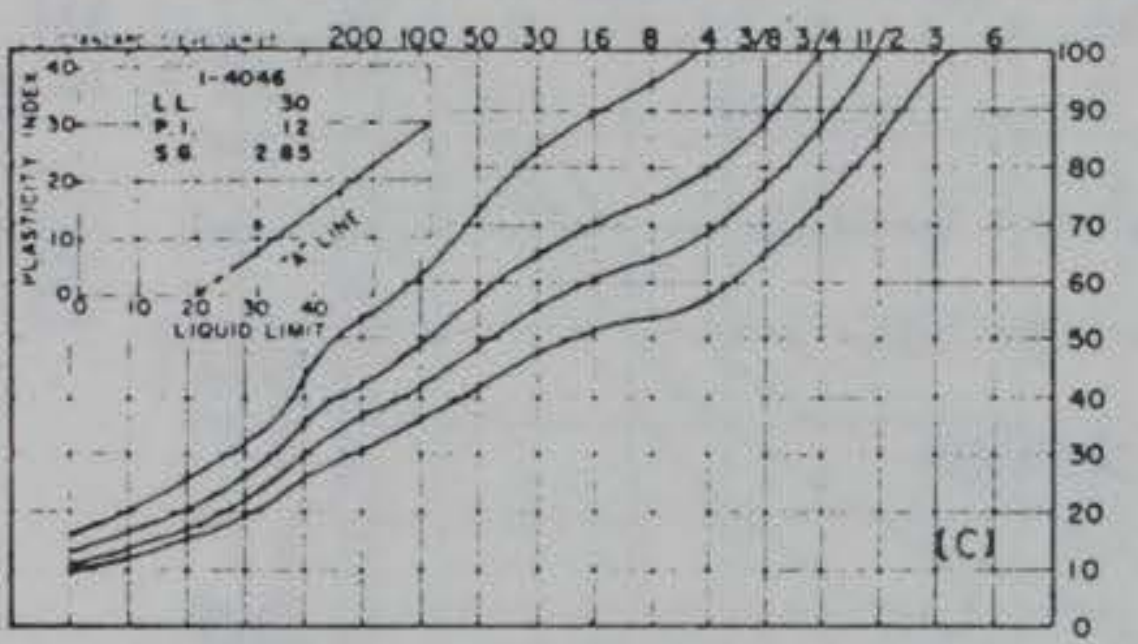

SIMILAR TO GRADING $B$ ON FIGURE 8

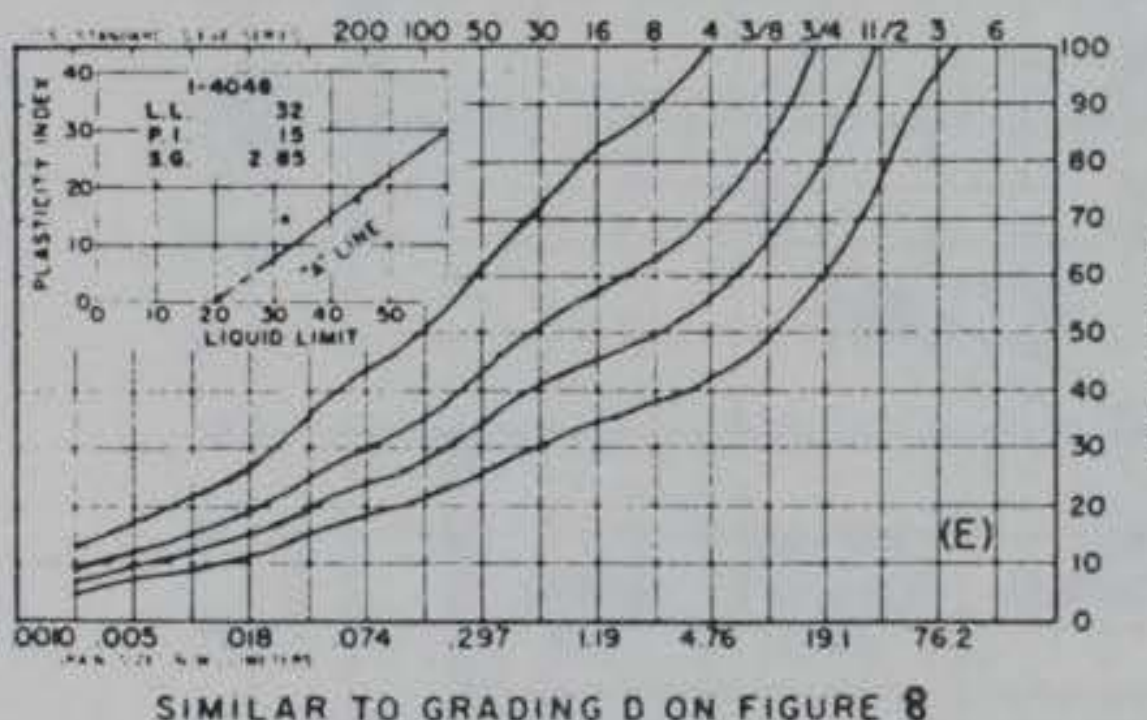

SIMILAR TO GRADING D ON FIGURE 8

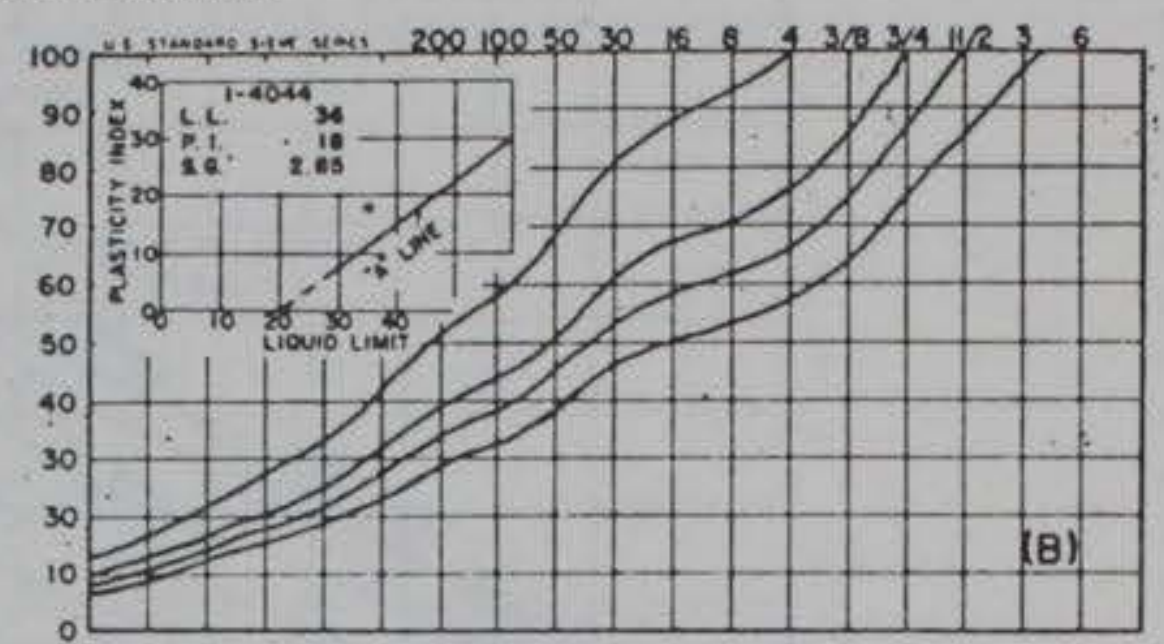

SIMILAR TO GRADING 8 ON FIGURE 8

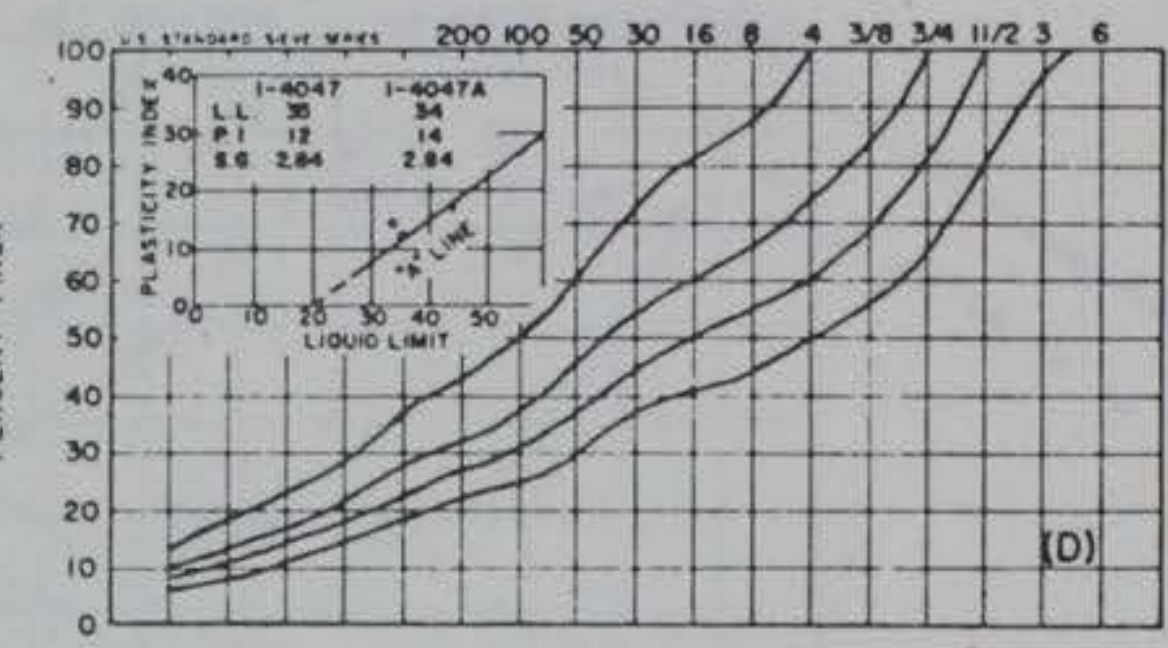

SIMILAR TO GRADING C ON FIGURE B

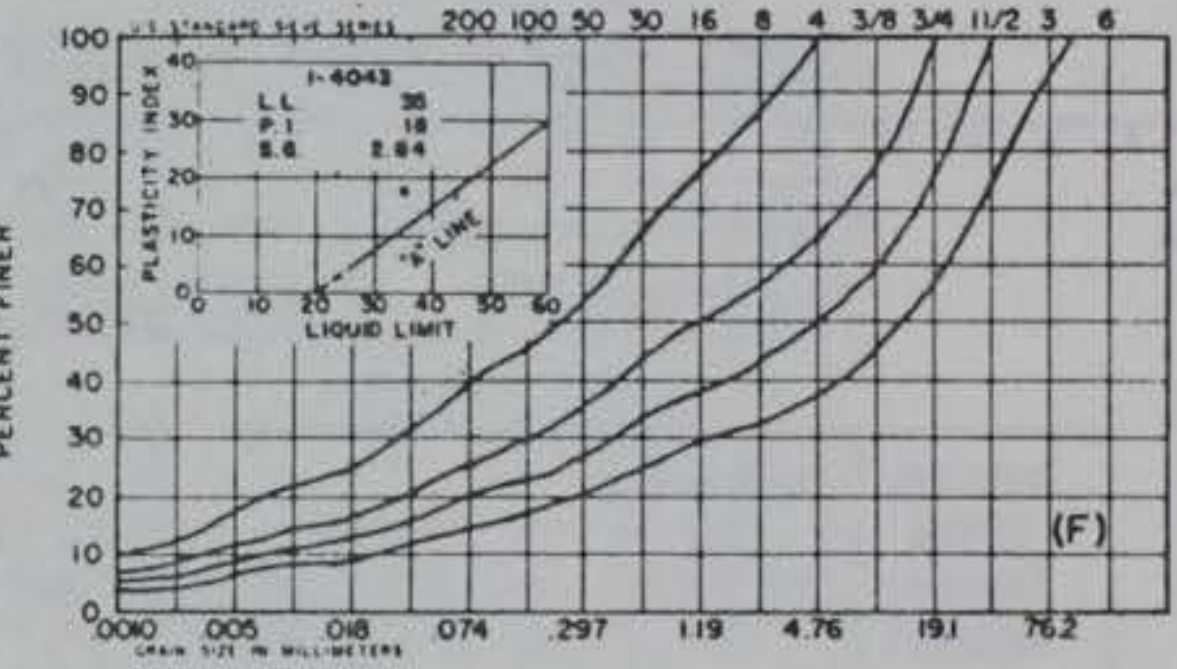

SIMILAR TO GRADING D ON FIGURE 8

Figure 18. Grading curves for compaction tests shown in Figure 19 (after Gordon, Hammond, and Miller 1964) 

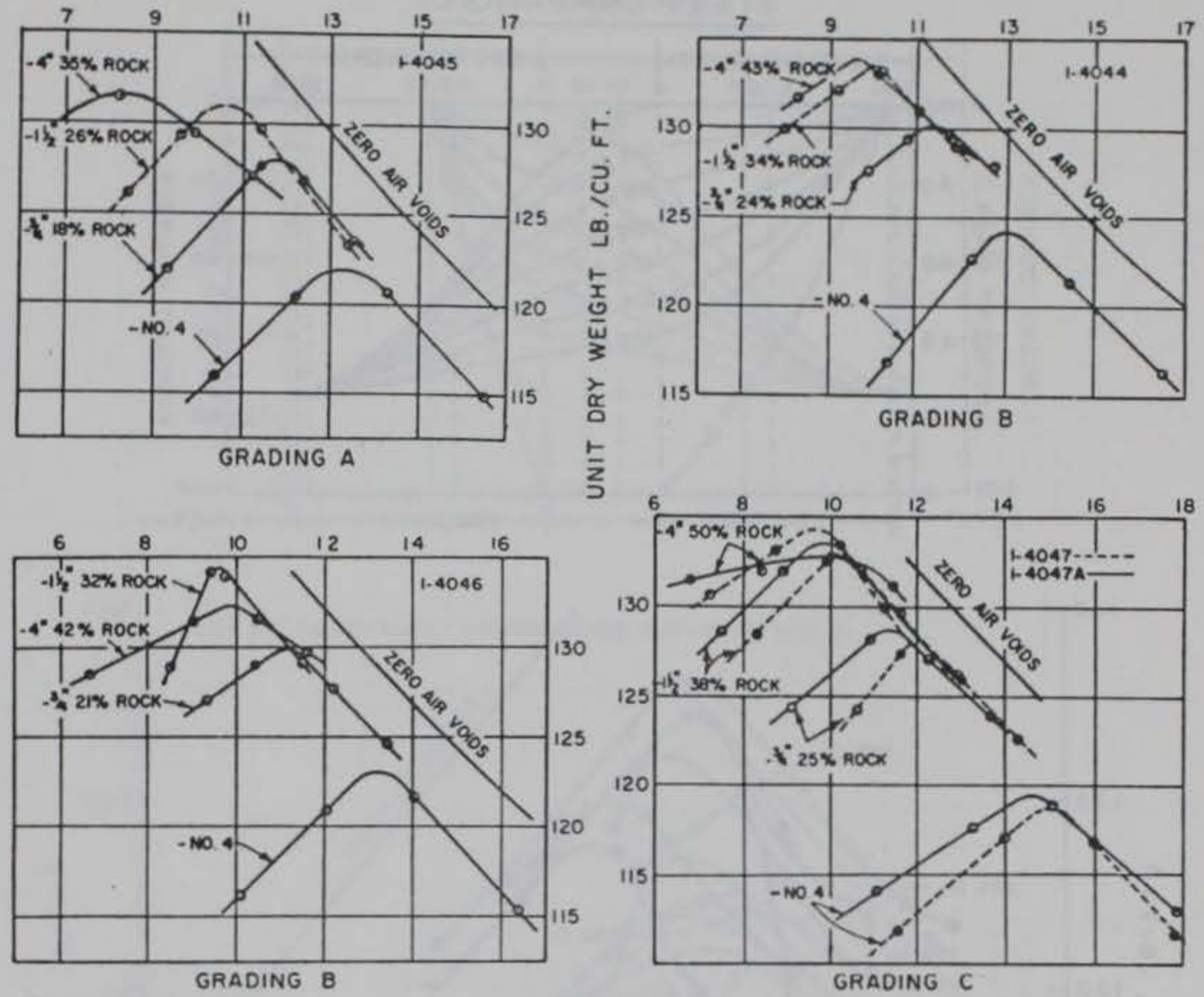

MOISTURE CONTENT IN PERCENT
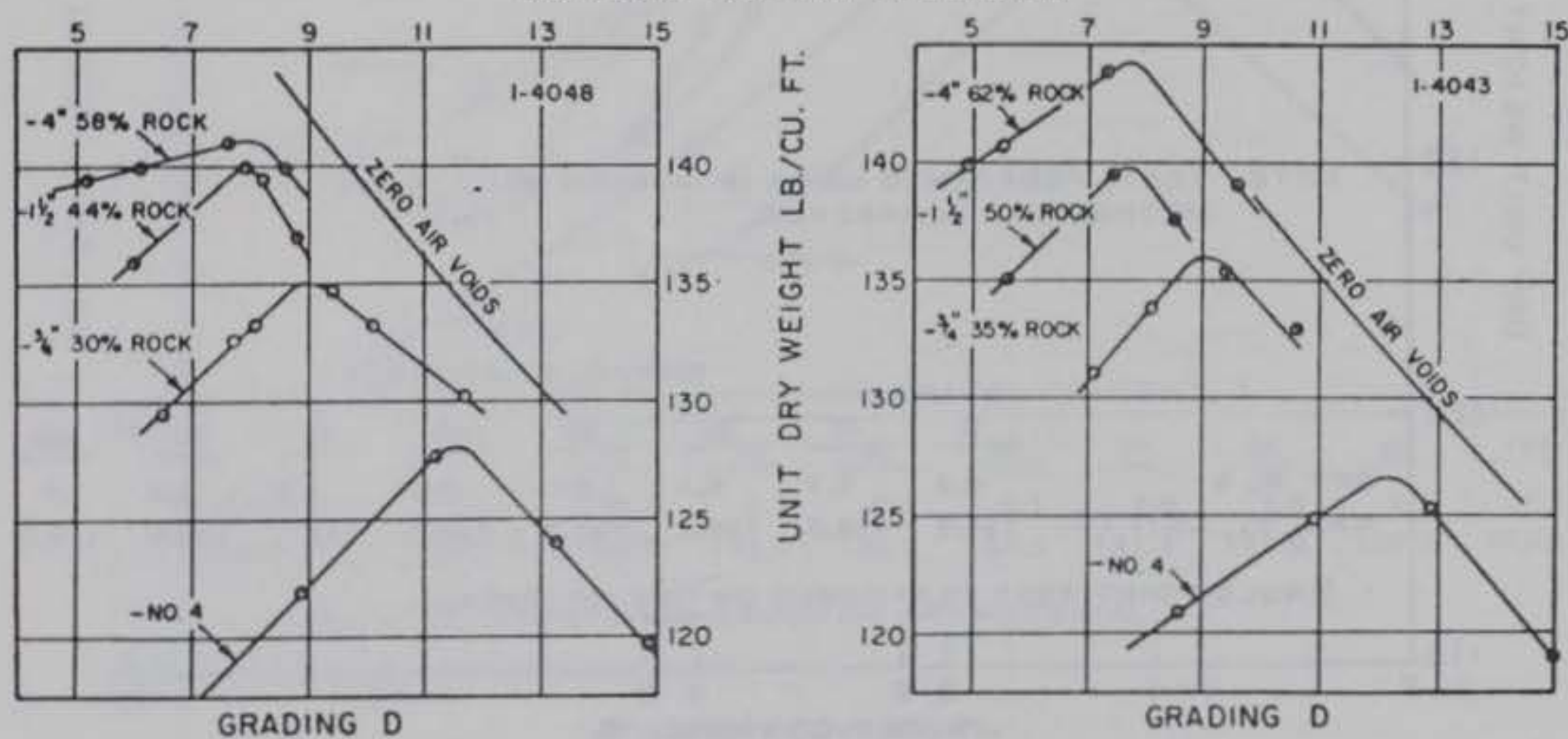

NOTE: $4 I N$ COMPOSITE; $200 \mathrm{CU} . \mathrm{FT}$. MOLD, 33 LB HAMMER

I $\frac{1}{2}$ IN. COMPOSITE : $.075 \mathrm{CU}$ FT. MOLD, 10 LO HAMMER

$\frac{3}{4}$ IN COMPOSITE: 075 CU. FT MOLO, 10 LO HAMMER

-NO 4 COMPOSITE: 0.05 CU.FT. MOLO, 10 LO HAMMER

Figure 19. Compaction characteristics of airport borrow material (after Gordon, Hammond, and Miller 1964) 


\section{TEST GRADATIONS}
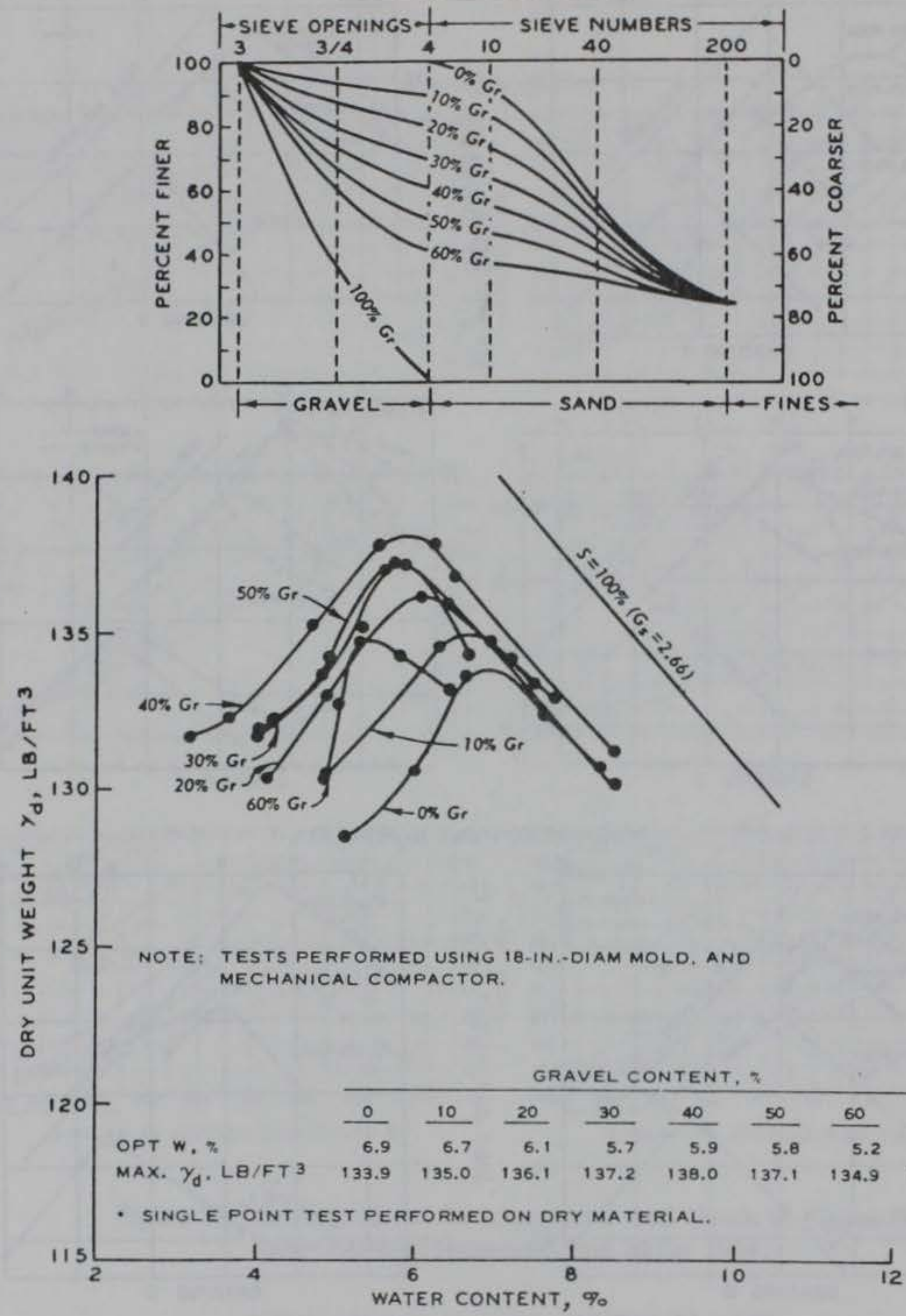

Figure 20. Compaction curves for conducted on full-scale specimens having variable gravel contents (after Donaghe and Townsend 1975) 

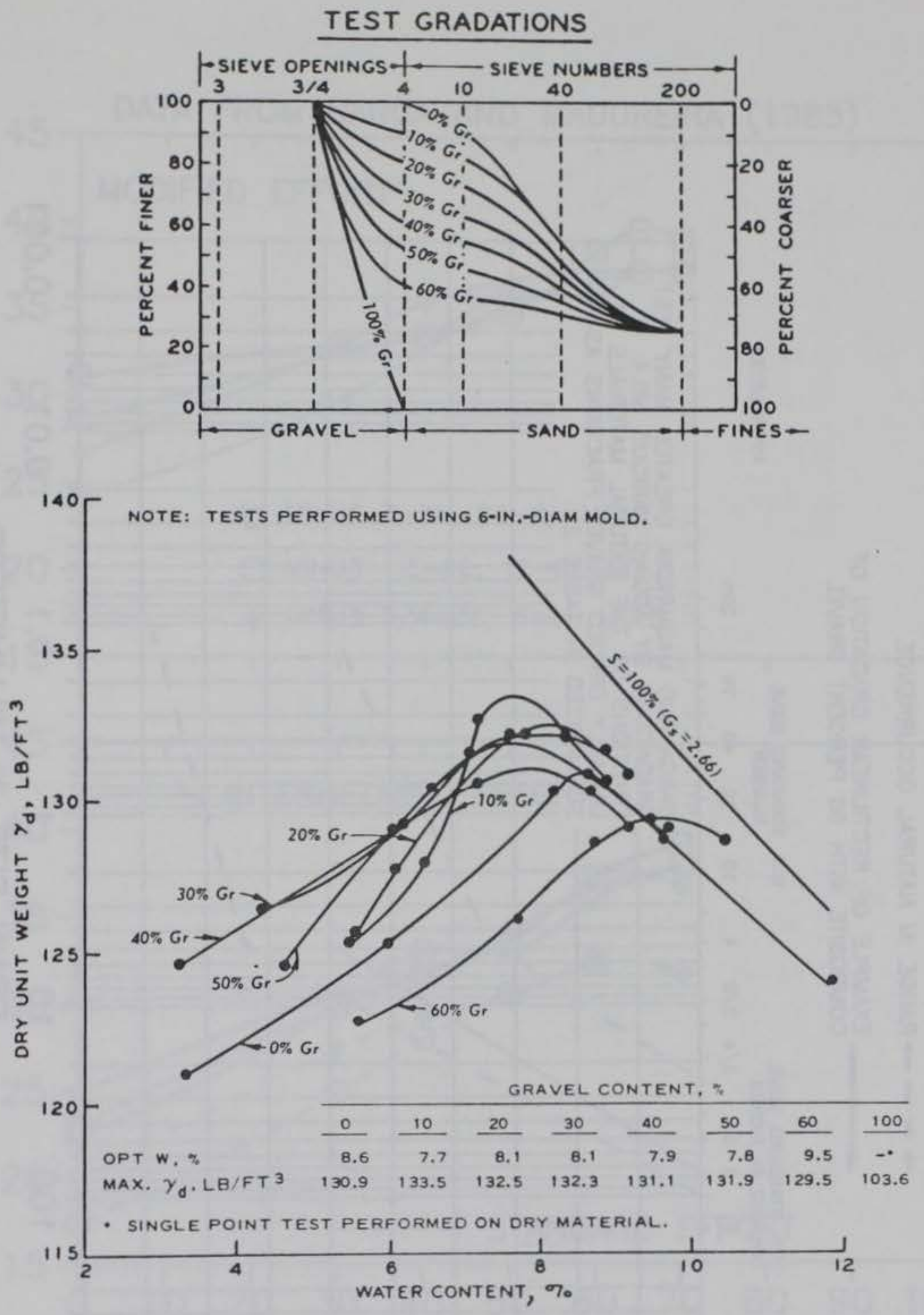

Figure 21. Compaction curves for small-scale tests conducted on scalped/replaced specimens corresponding to gradations of Figure 20 (after Donaghe and Townsend 1975) 
GARGA AND MADUREIRA (1985)

- - - - RANGE IN NATURAL OCCURRENCE EXAMPLE OF RECTILINEAR GRADATION OF COMPOSITE WITH 60 PERCENT GRAVEL

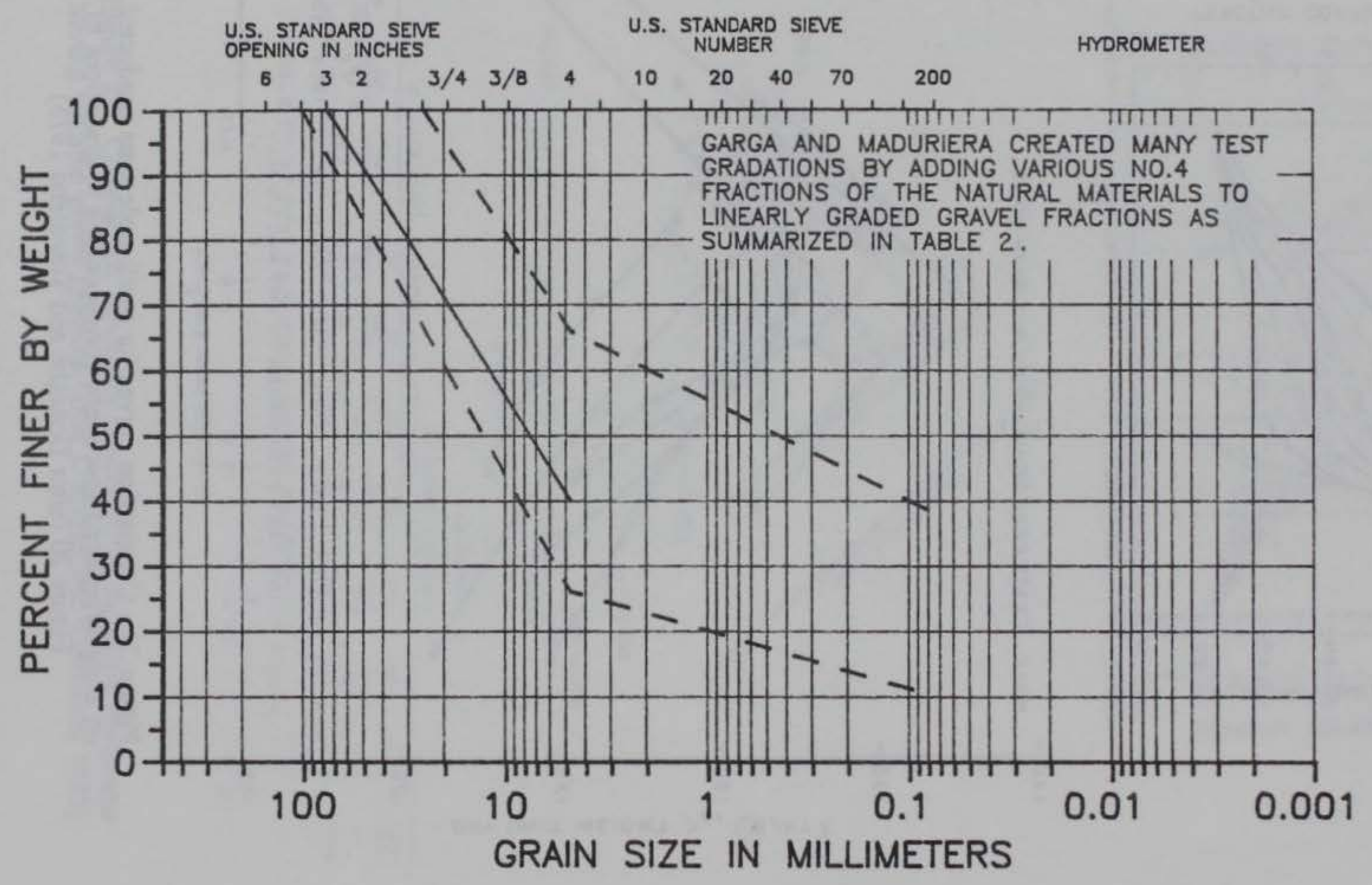

Figure 22. Range in grain-size distributions (after Garga and Madureira 1985) 

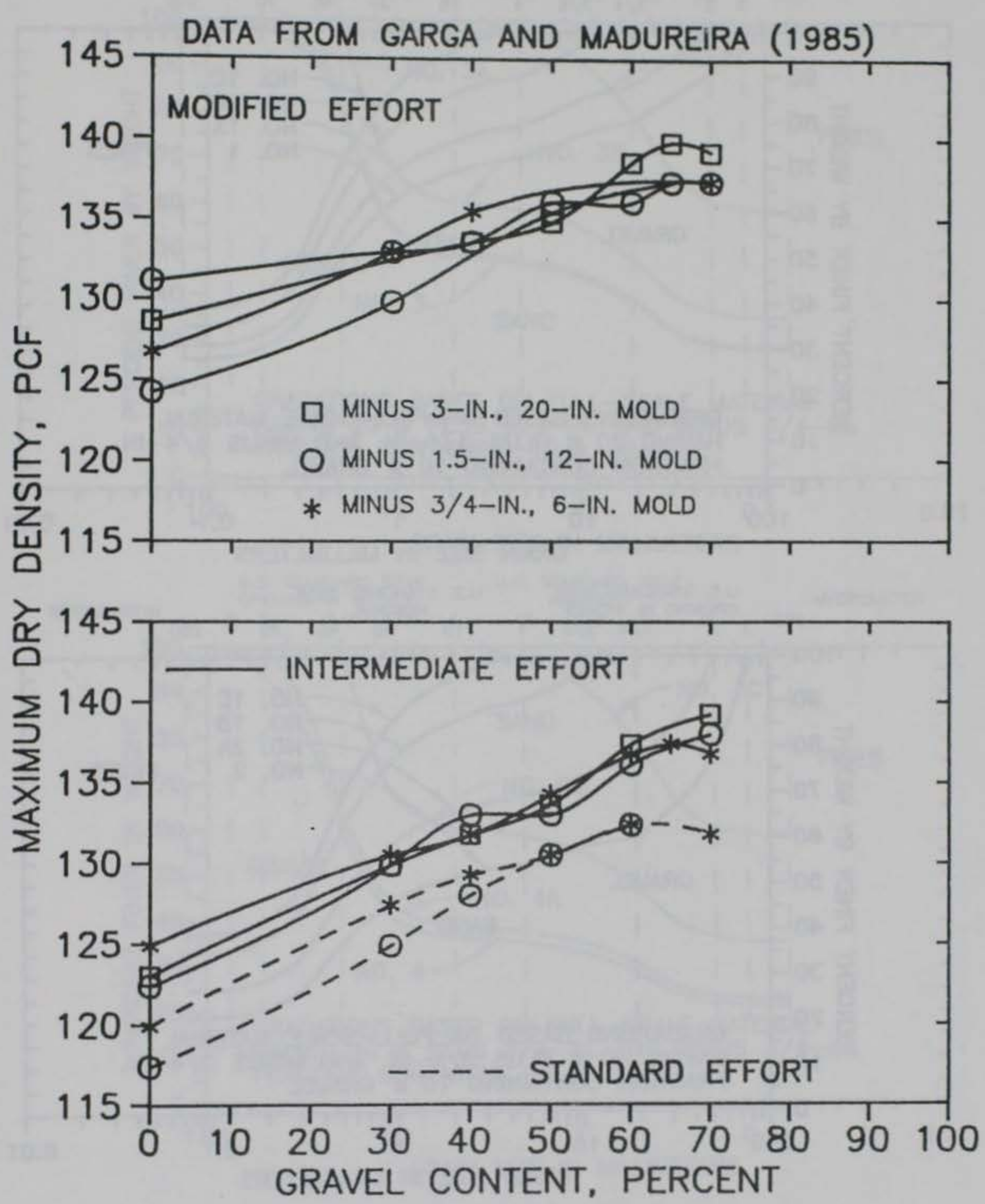

Figure 23. Influence of equipment size on maximum dry density at standard, intermediate, and modified compaction efforts (after Garga and Madureira 1985) 

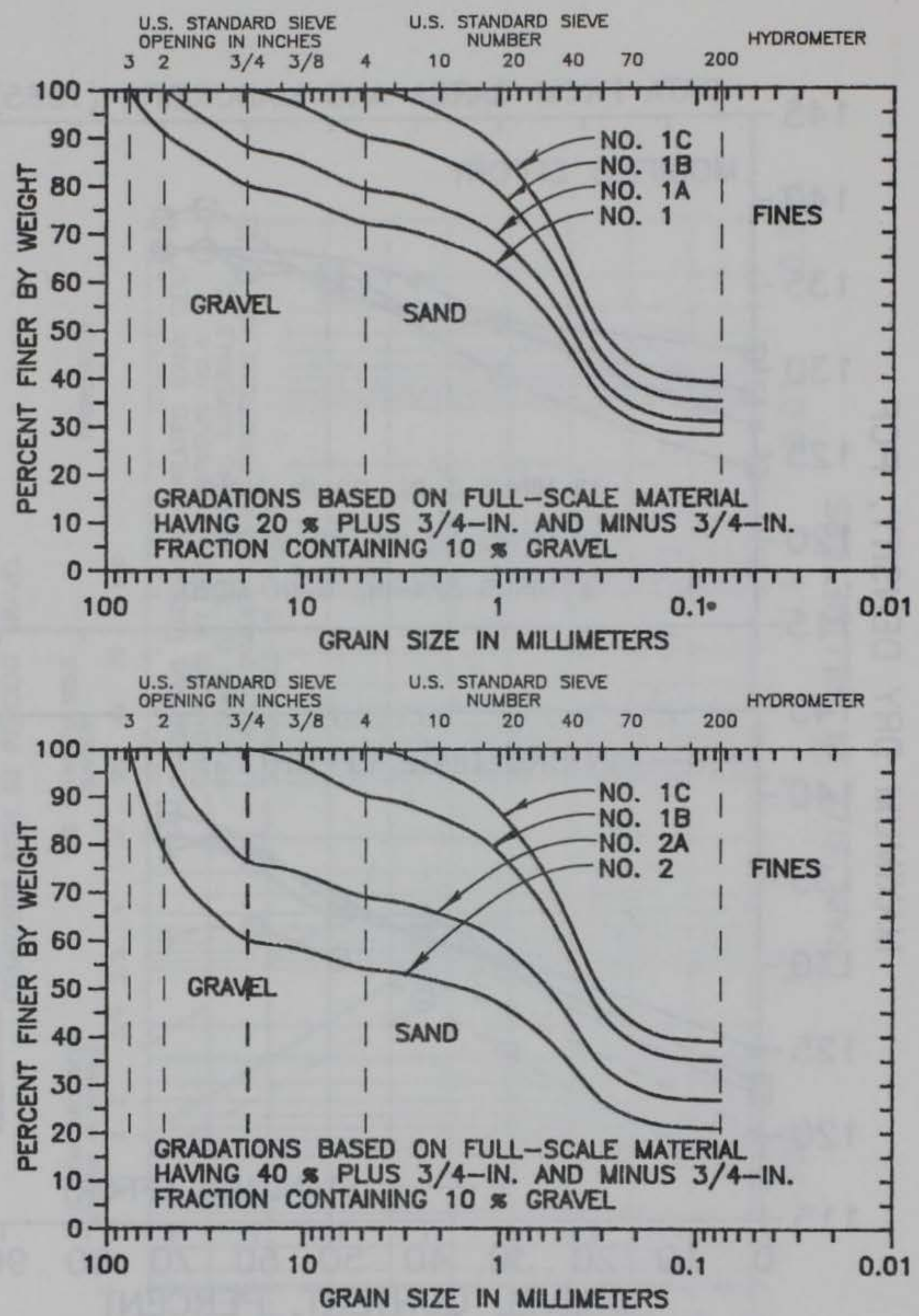

Figure 24. Minus 3-in. full-scale test gradation Nos. 1 and 2 and their fractions (after Torrey and Donaghe 1991) 

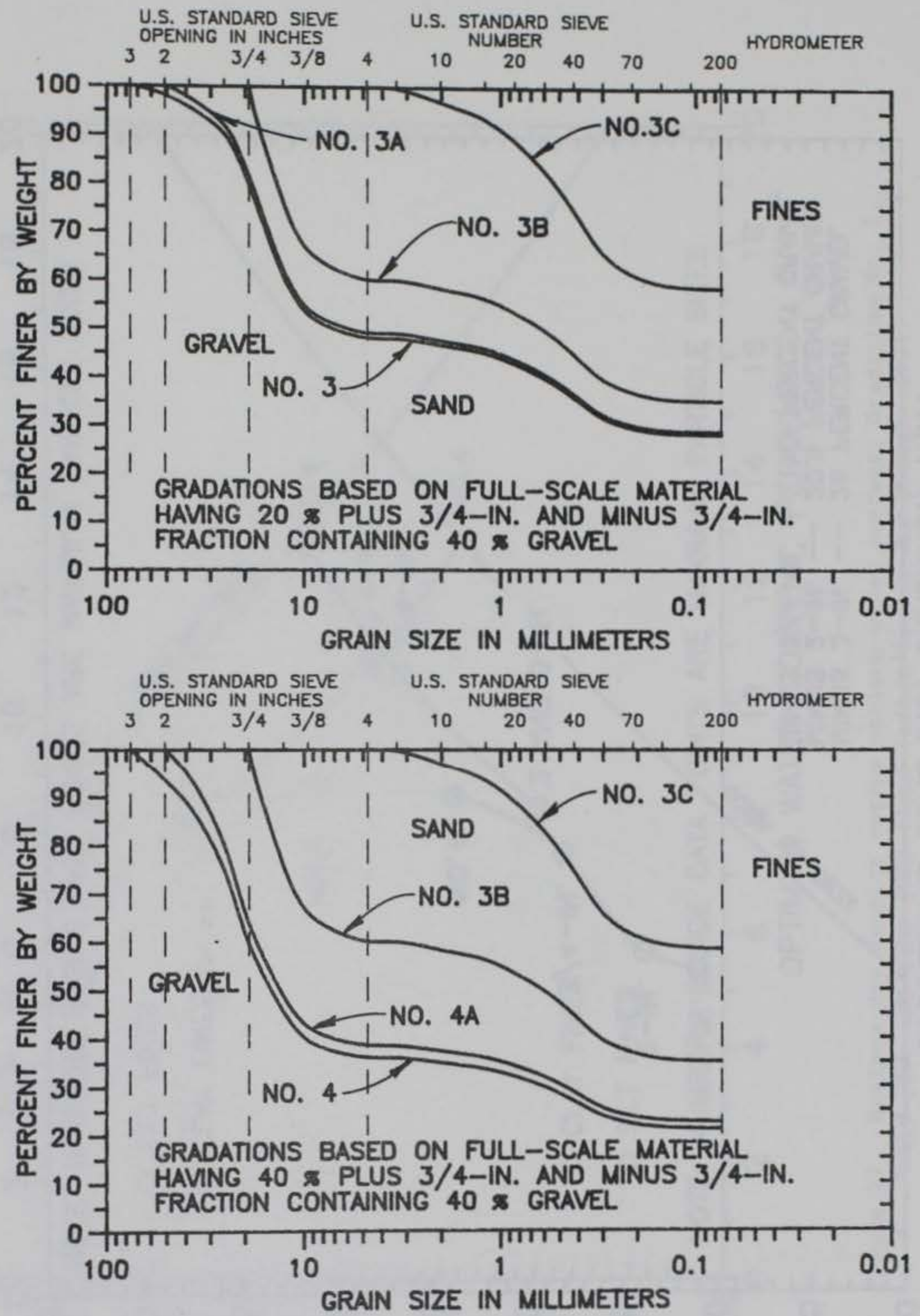

Figure 25. Minus 3-in. full-scale test gradation Nos. 3 and 4 and their fractions (after Torrey and Donaghe 1991) 
SUCCESSIVE SCALPING, GRADATION NO.1

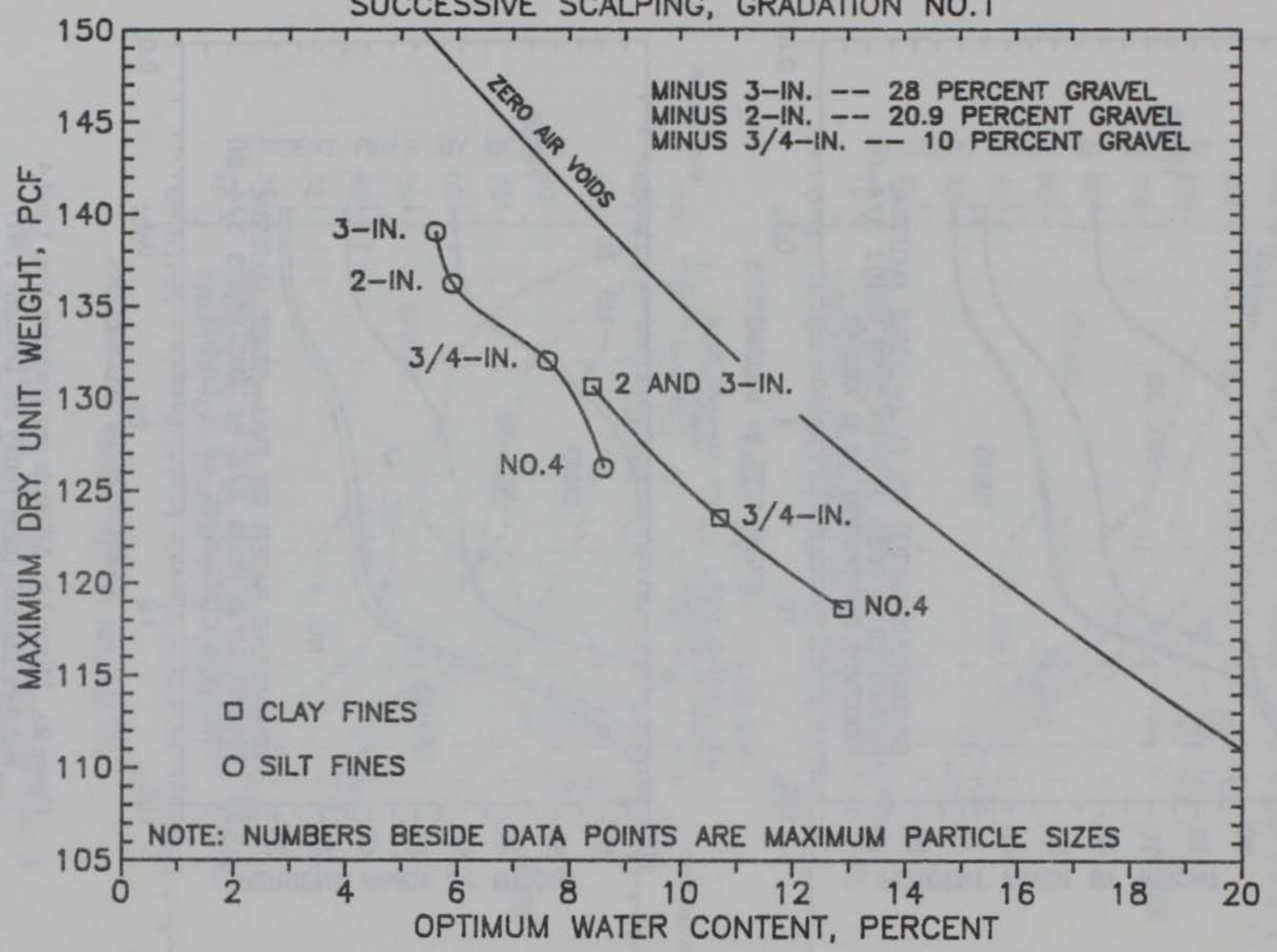

Figure 26. Maximum dry density versus optimum water content, Gradation No. 1, silt (ML) and clay (CH) fines (after Torrey and Donaghe 1991) 


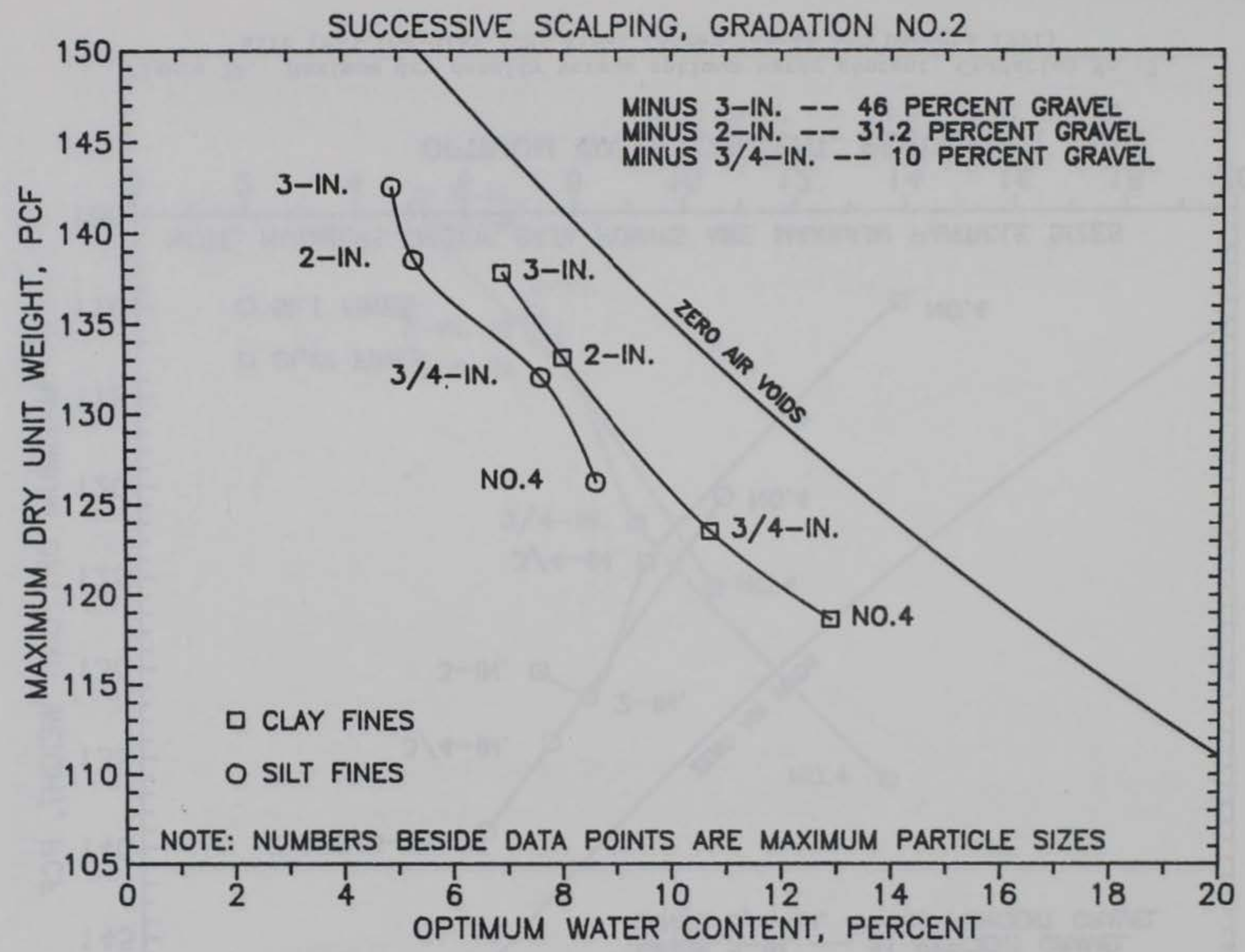

Figure 27. Maximum dry density versus optimum water content, Gradation No. 2, silt (ML) and clay (CH) fines (after Torrey and Donaghe 1991) 


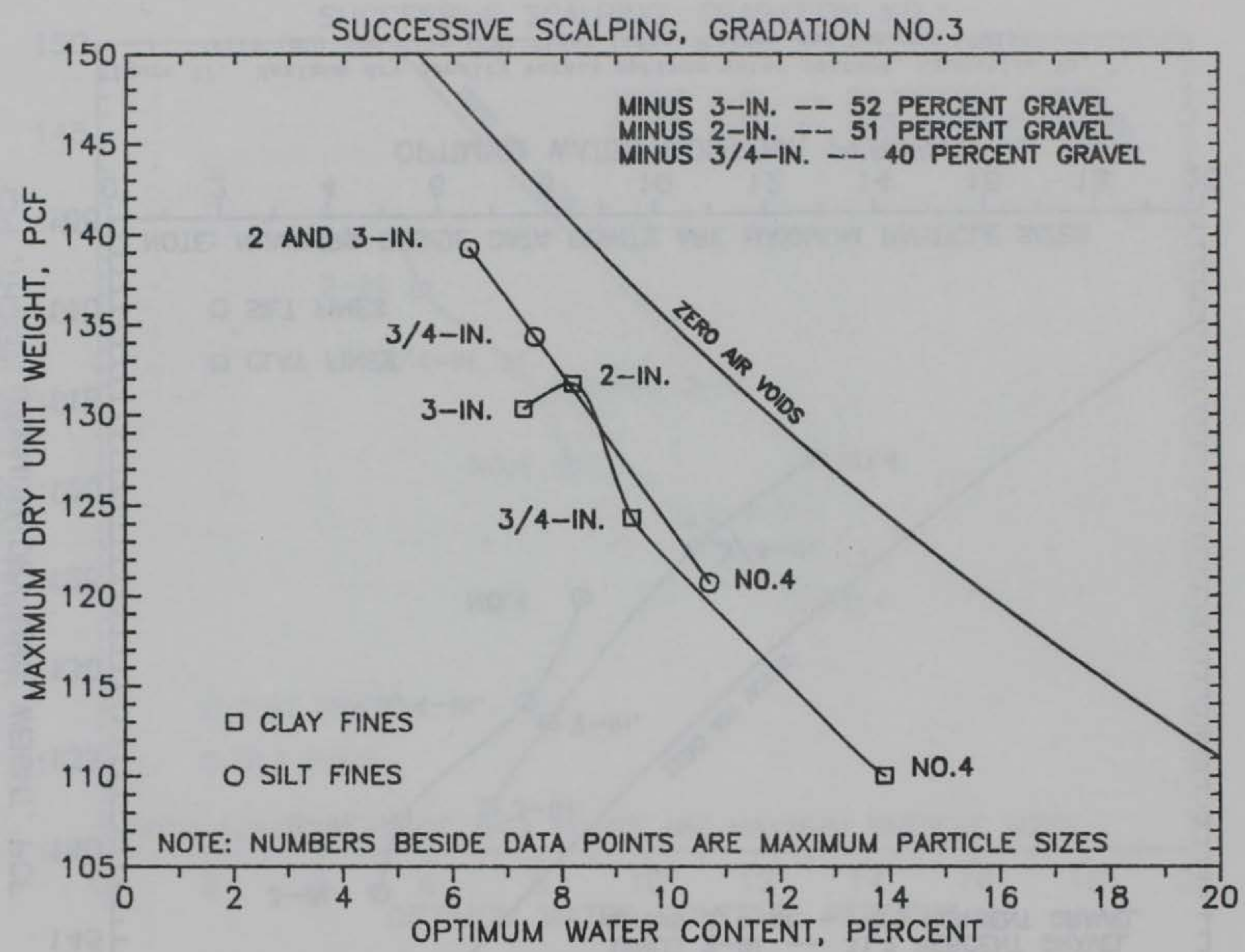

Figure 28. Maximum dry density versus optimum water content, Gradation No. 3, silt (ML) and clay (CH) fines (after Torrey and Donaghe 1991) 
SUCCESSIVE SCALPING, GRADATION NO.4

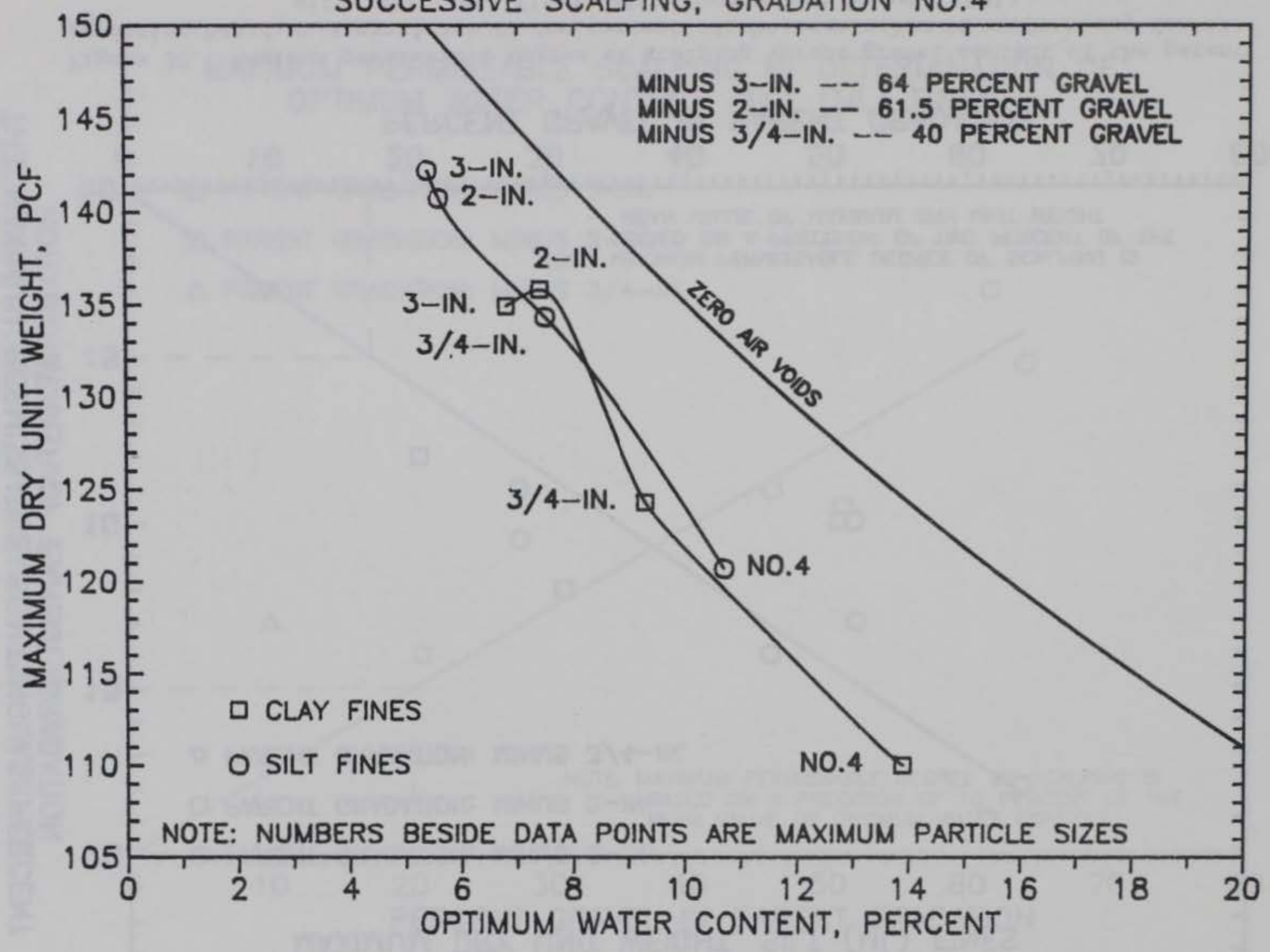

Figure 29. Maximum dry density versus optimum water content, Gradation No. 4, silt (ML) and clay (CH) fines (after Torrey and Donaghe 1991) 


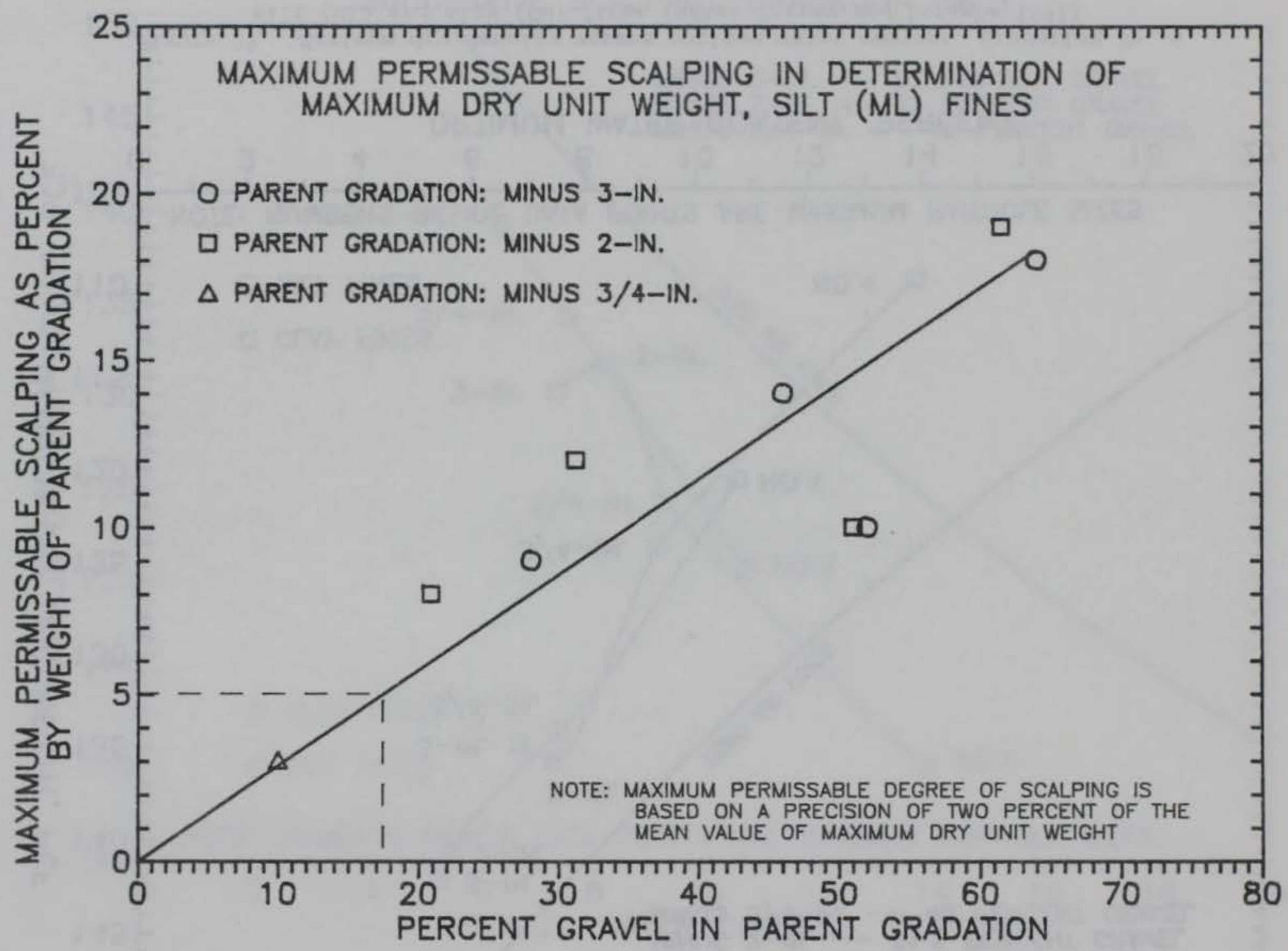

Figure 30. Maximum permissible degree of scalping versus gravel content of the parent gradation based on a precision of two percent of the mean value of maximum dry density, silt (ML) fines (after Torrey and Donaghe 1991) 


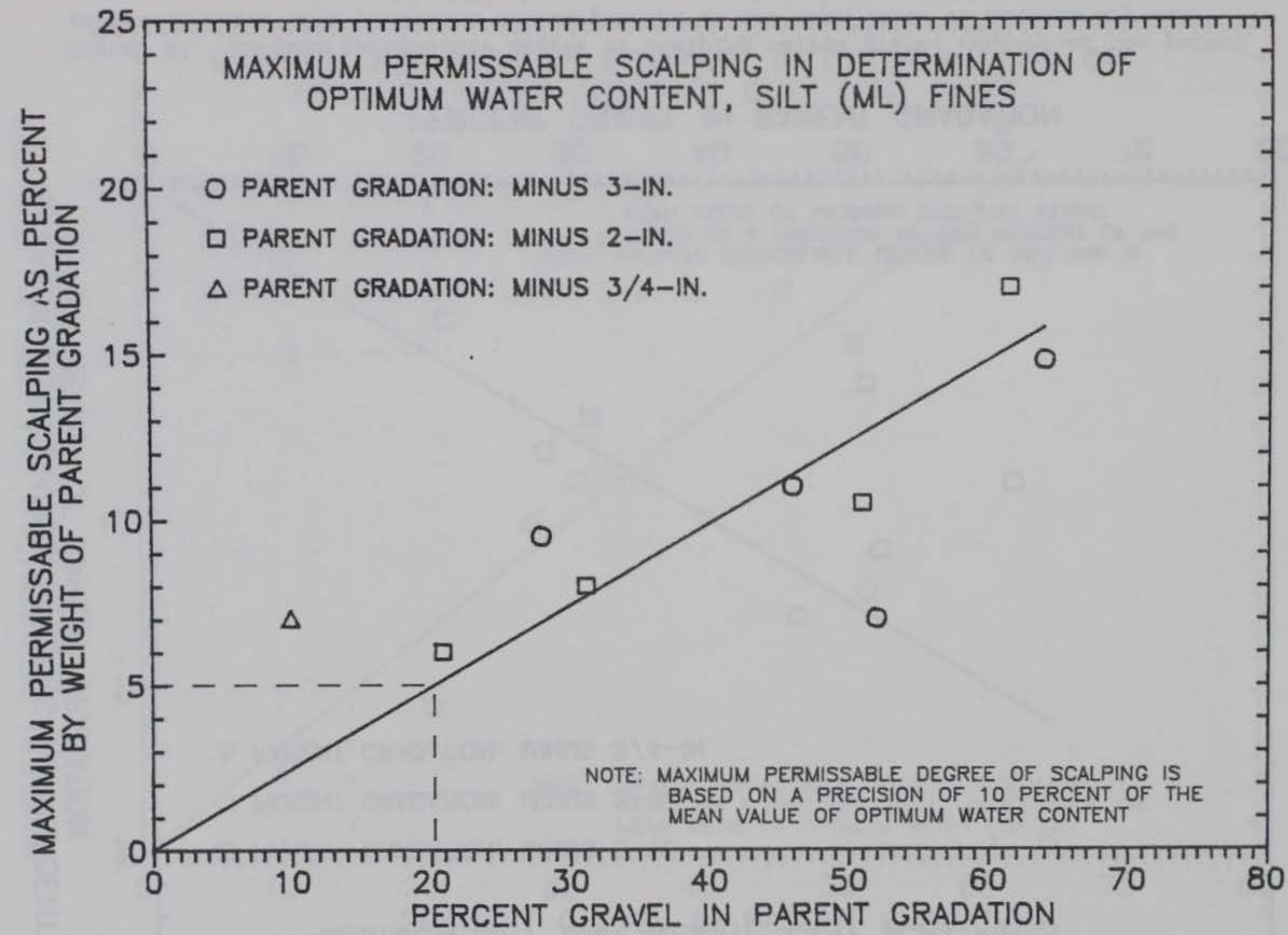

Figure 31. Maximum permissible degree of scalping versus gravel content of the parent gradation based on a precision of ten percent of the mean value of optimum water con-

tent, silt (ML) fines (after Torrey and Donaghe 1991) 


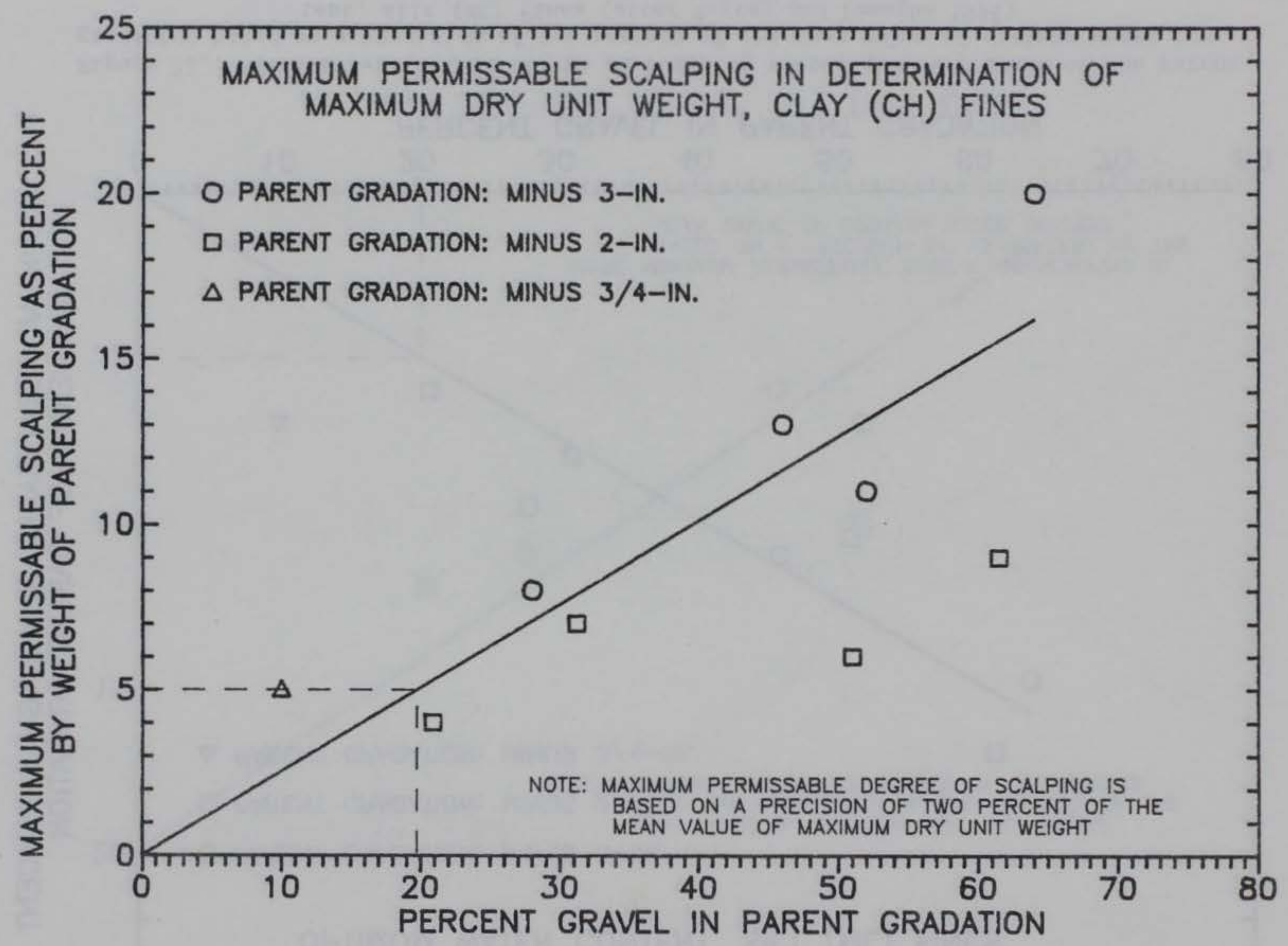

Figure 32. Maximum permissible degree of scalping versus gravel content of the parent gradation based on a precision of two percent of the mean value of maximum dry density, clay (CH) fines (after Torrey and Donaghe 1991) 


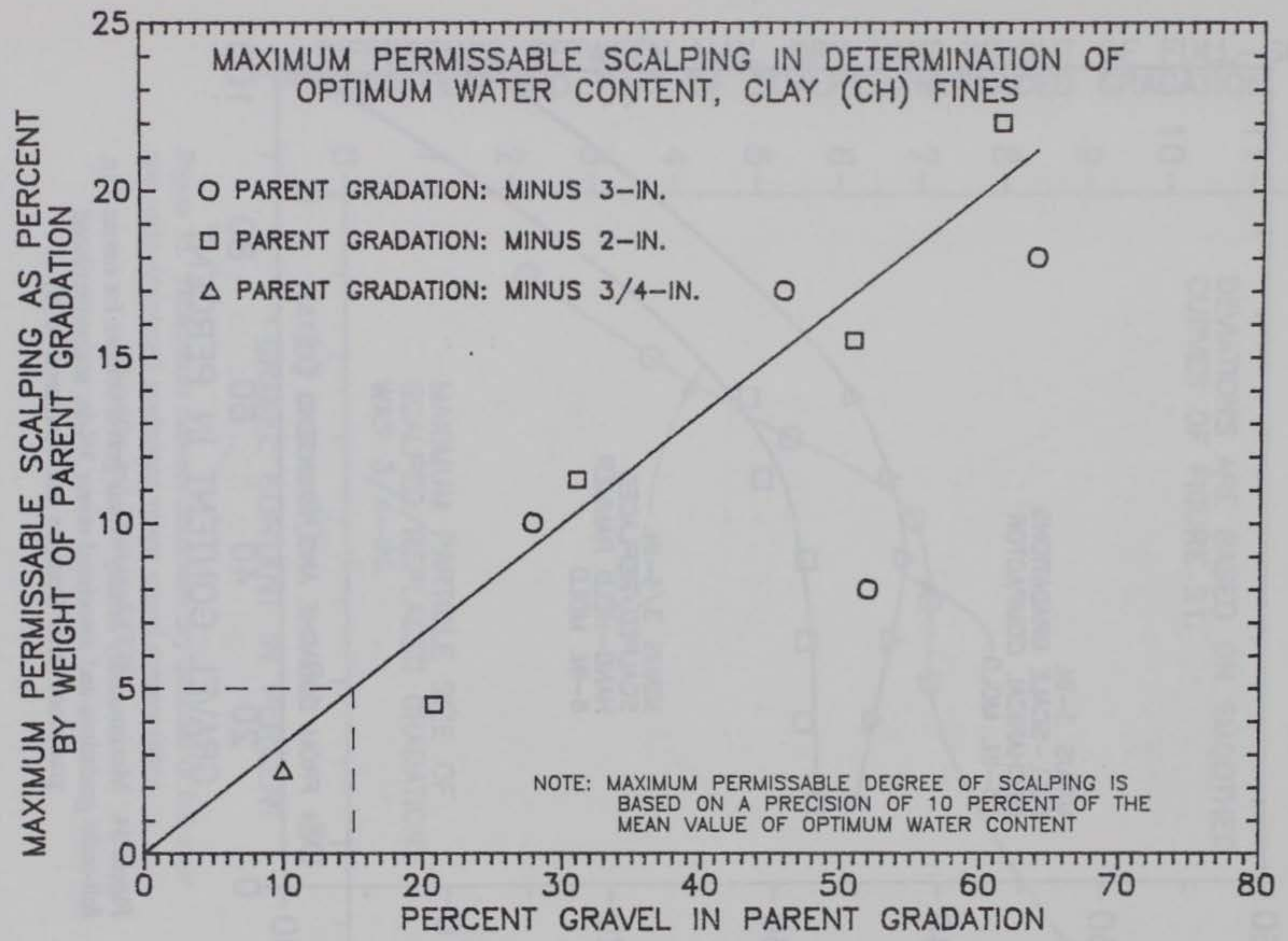

Figure 33. Maximum permissible degree of scalping versus gravel content of the parent gradation based on a precision of 10 percent of the mean value of optimum water content, clay (CH) fines (after Torry and Donaghe 1991) 


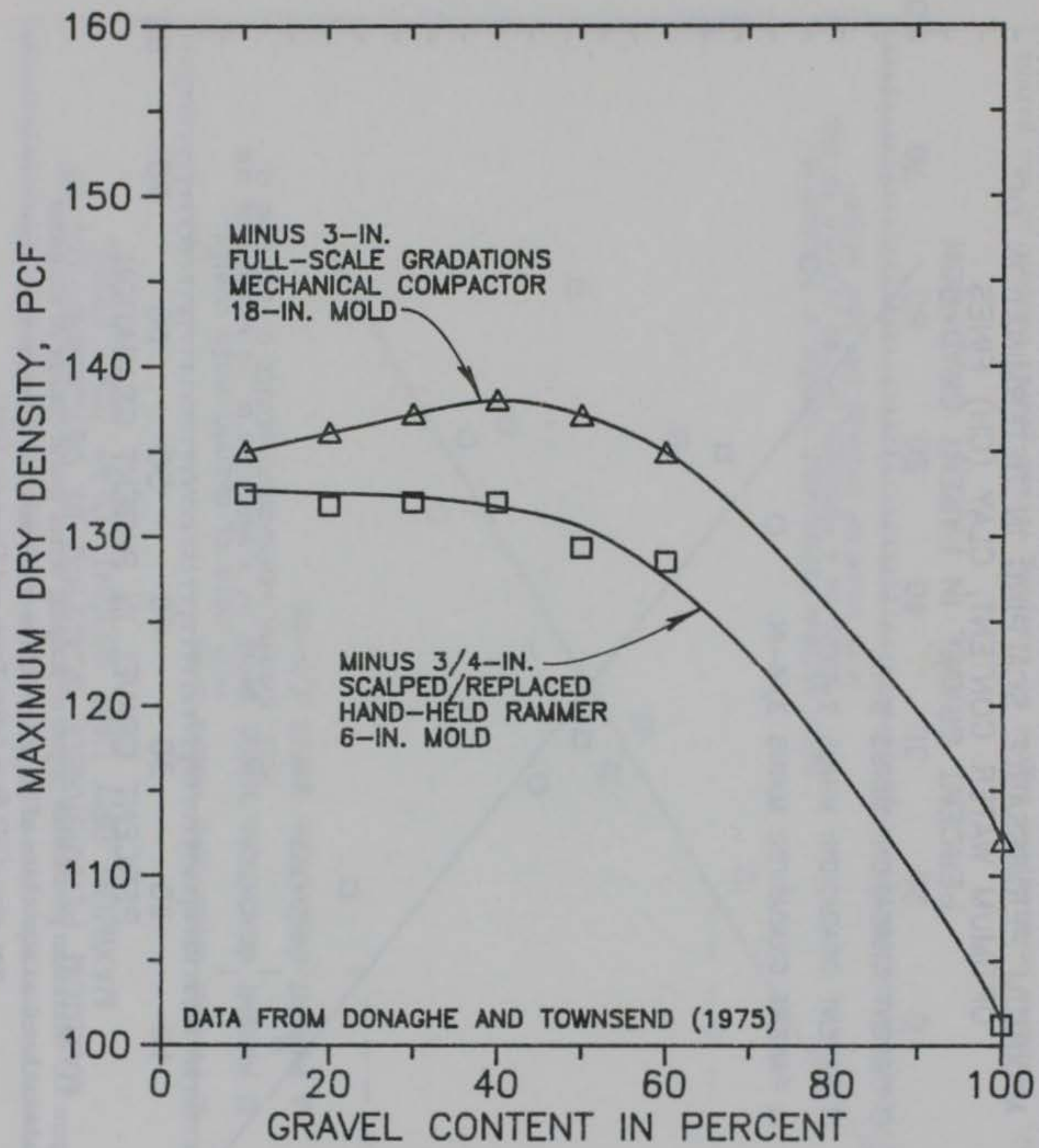

Figure 34. Maximum dry density versus gravel content for minus 3-in. full-scale gradations and associated minus 3/4-in. scalped/replaced gradations (after Donaghe and Townsend 1975) 


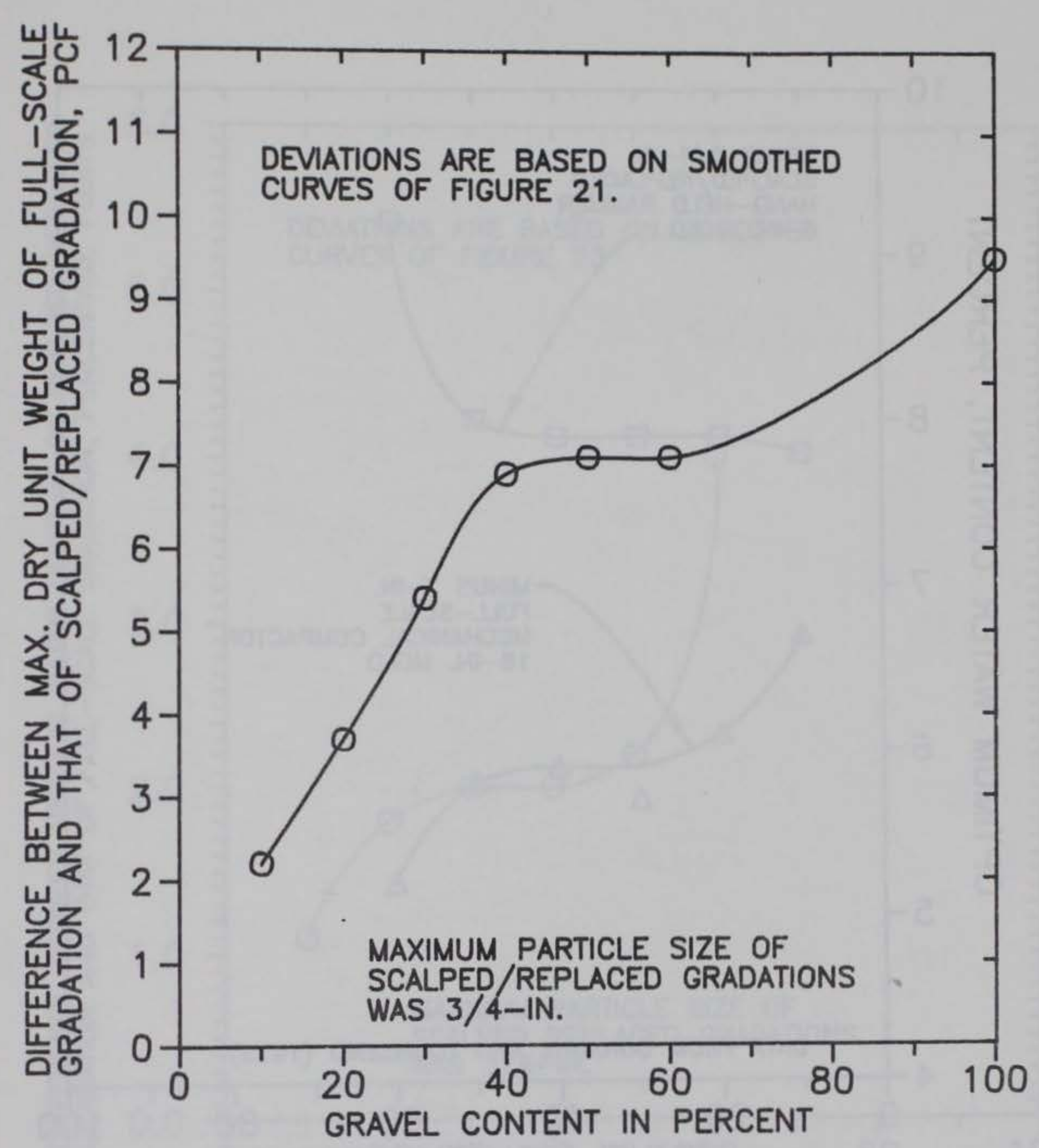

Figure 35. Differences between maximum dry densities of full-scale and scalped/replaced gradations versus gravel content (after Donaghe and Townsend 1975) 


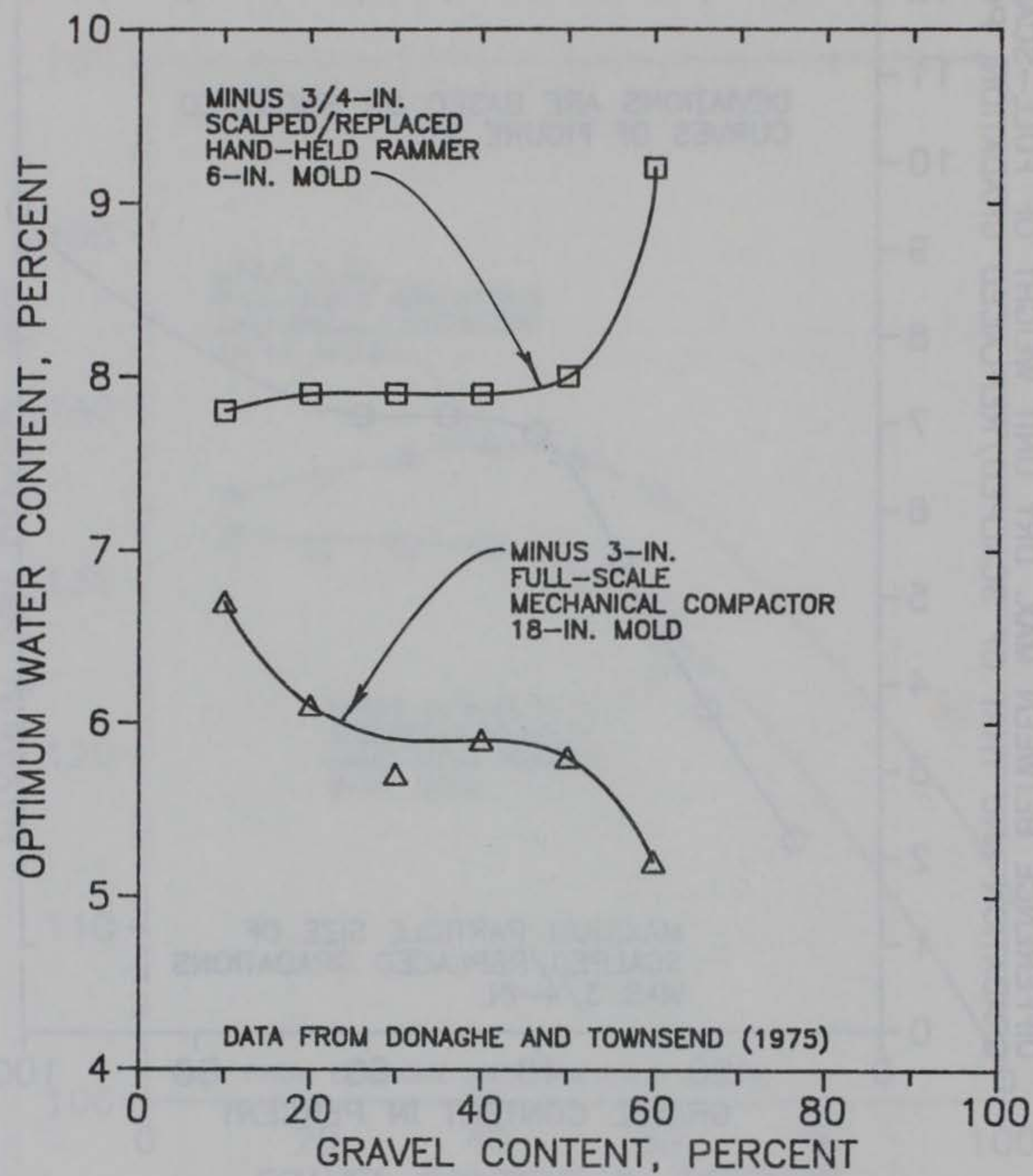

Figure 36. Optimum water content versus gravel content for minus 3 -in. full-scale gradations and associated minus 3/4-in. scalped and replaced gradations (after Donaghe and Townsend 1975) 


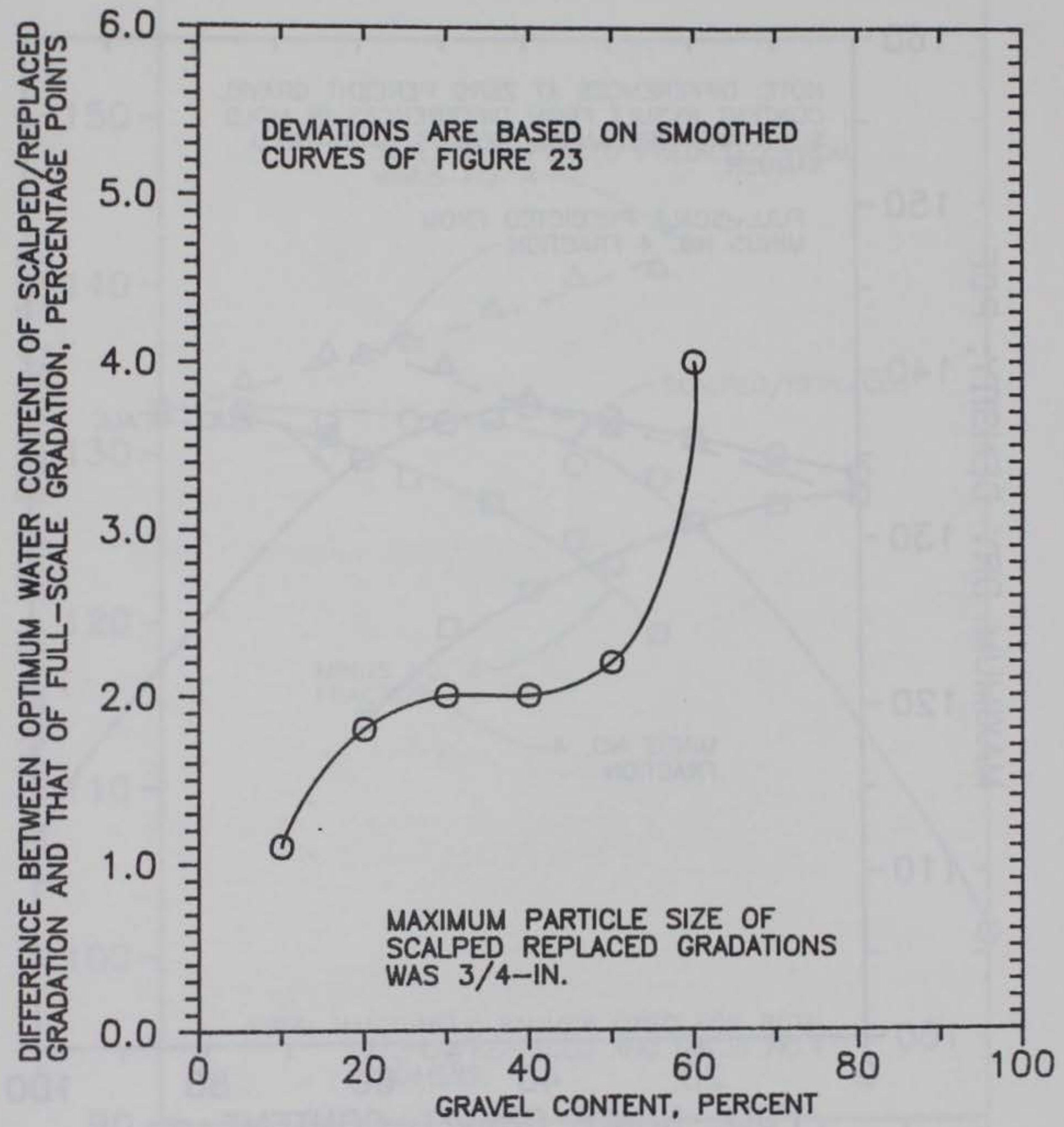

Figure 37. Differences between optimum water contents of full-scale and scalped/replaced gradations versus gravel content (after Donaghe and Townsend 1975) 


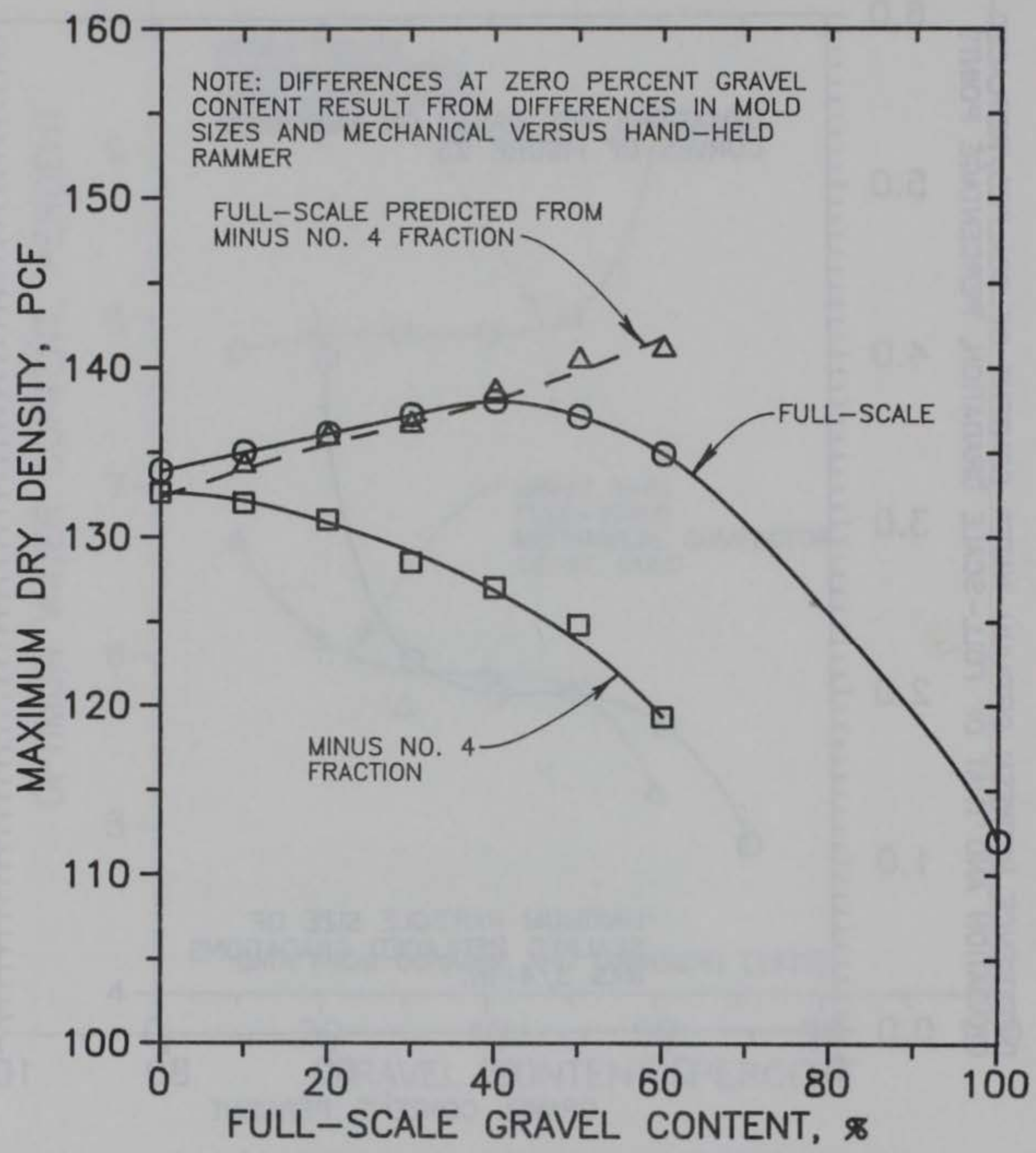

Figure 38. Predictions of maximum dry densities of minus 3-in. full-scale gradations from maximum dry densities of their minus No. 4 fractions using Equation 3 (after Donaghe and Townsend 1975) 


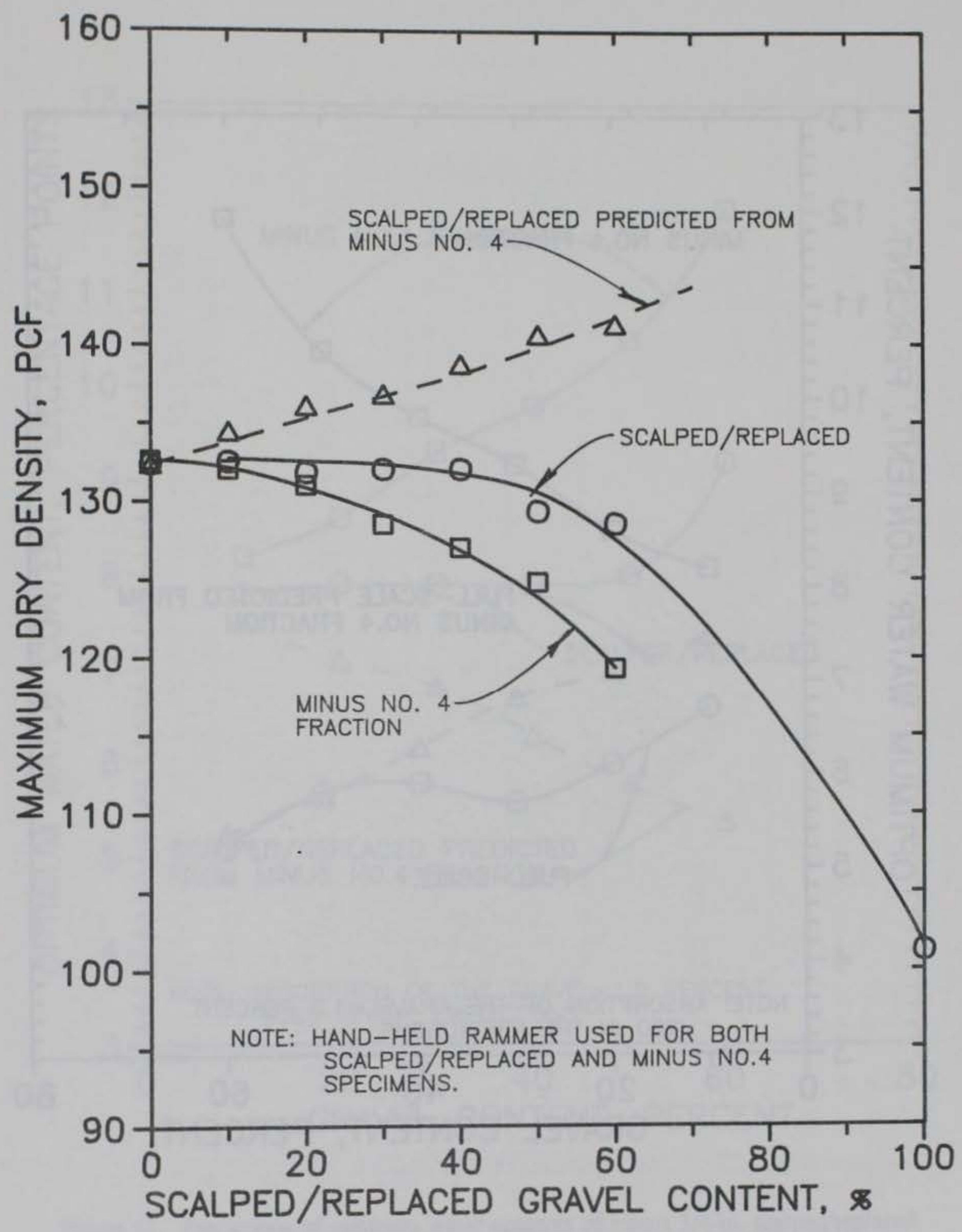

Figure 39. Predictions of maximum dry densities of minus 3/4-in. scalped/replaced gradations from maximum dry densities of their minus No. 4 fractions using Equation 3 (after Donaghe and Townsend 1975) 


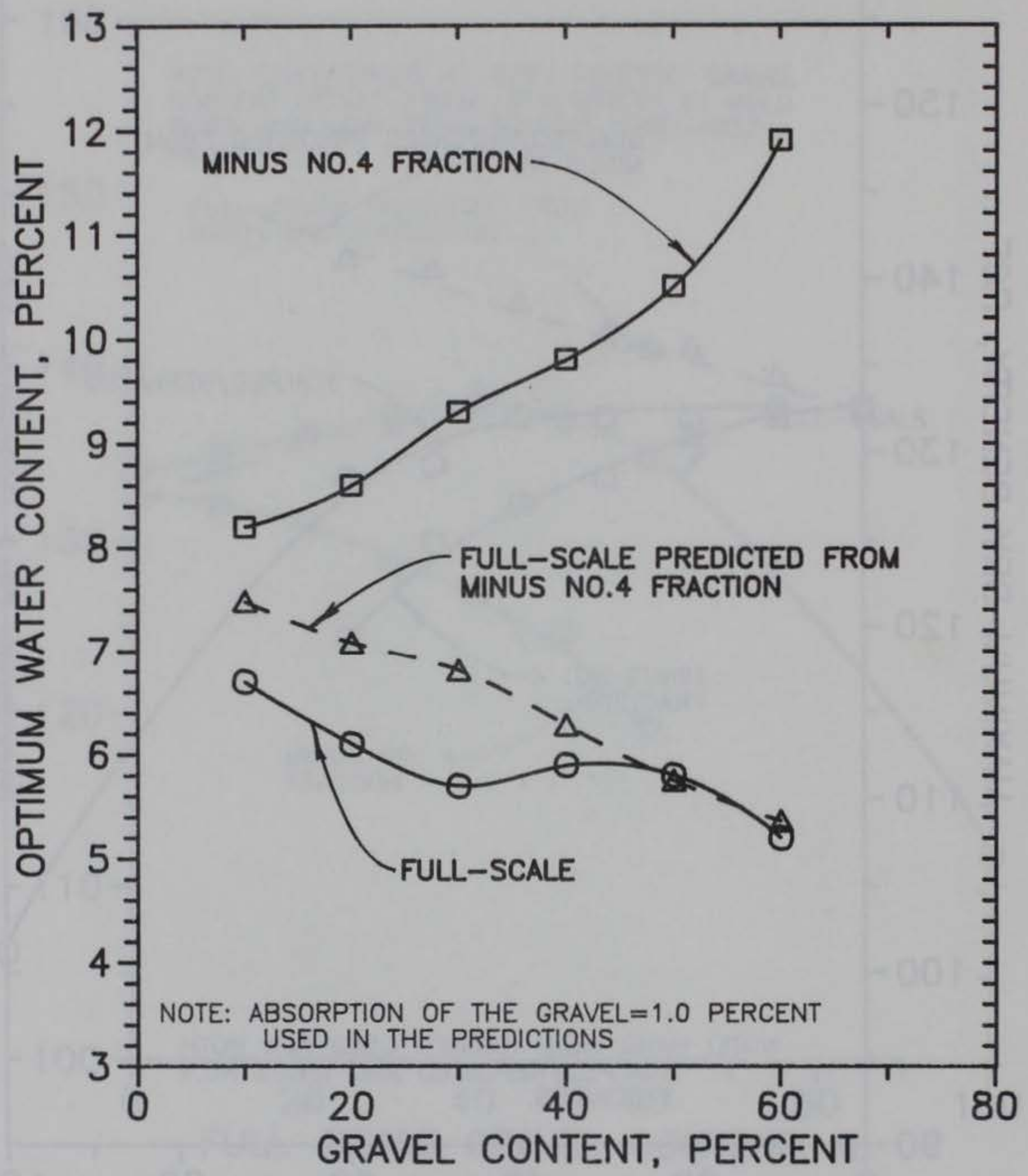

Figure 40. Predictions of optimum water contents of minus 3-in. full-scale gradations from optimum water contents of their minus No. 4 fractions using Equation 4 (after Donaghe and Townsend 1975) 


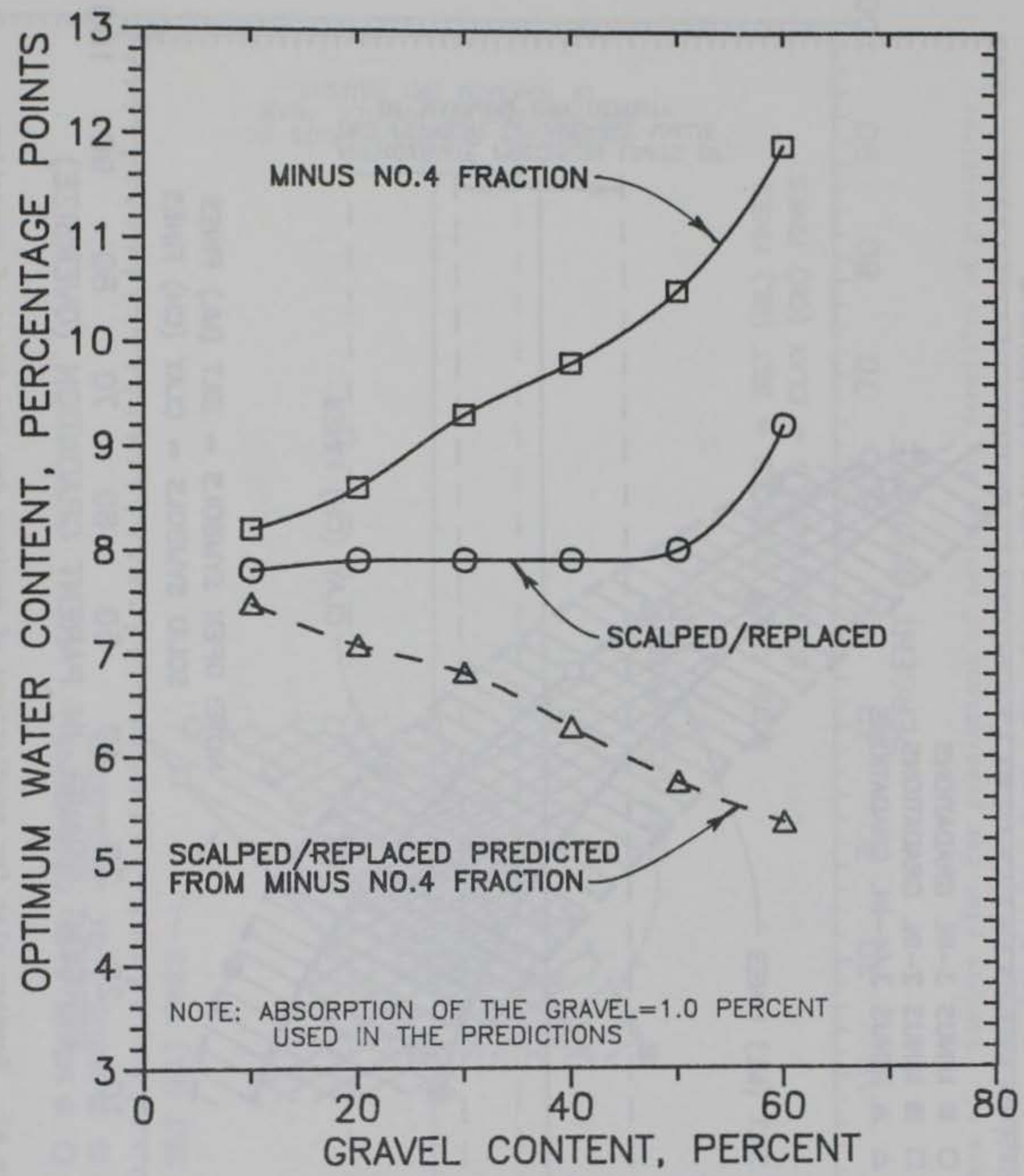

Figure 41. Predictions of optimum water contents of minus 3/4-in. scalped/replaced gradations from optimum water contents of their minus No. 4 fractions using Equation 4 (after Donaghe and Townsend 1975) 


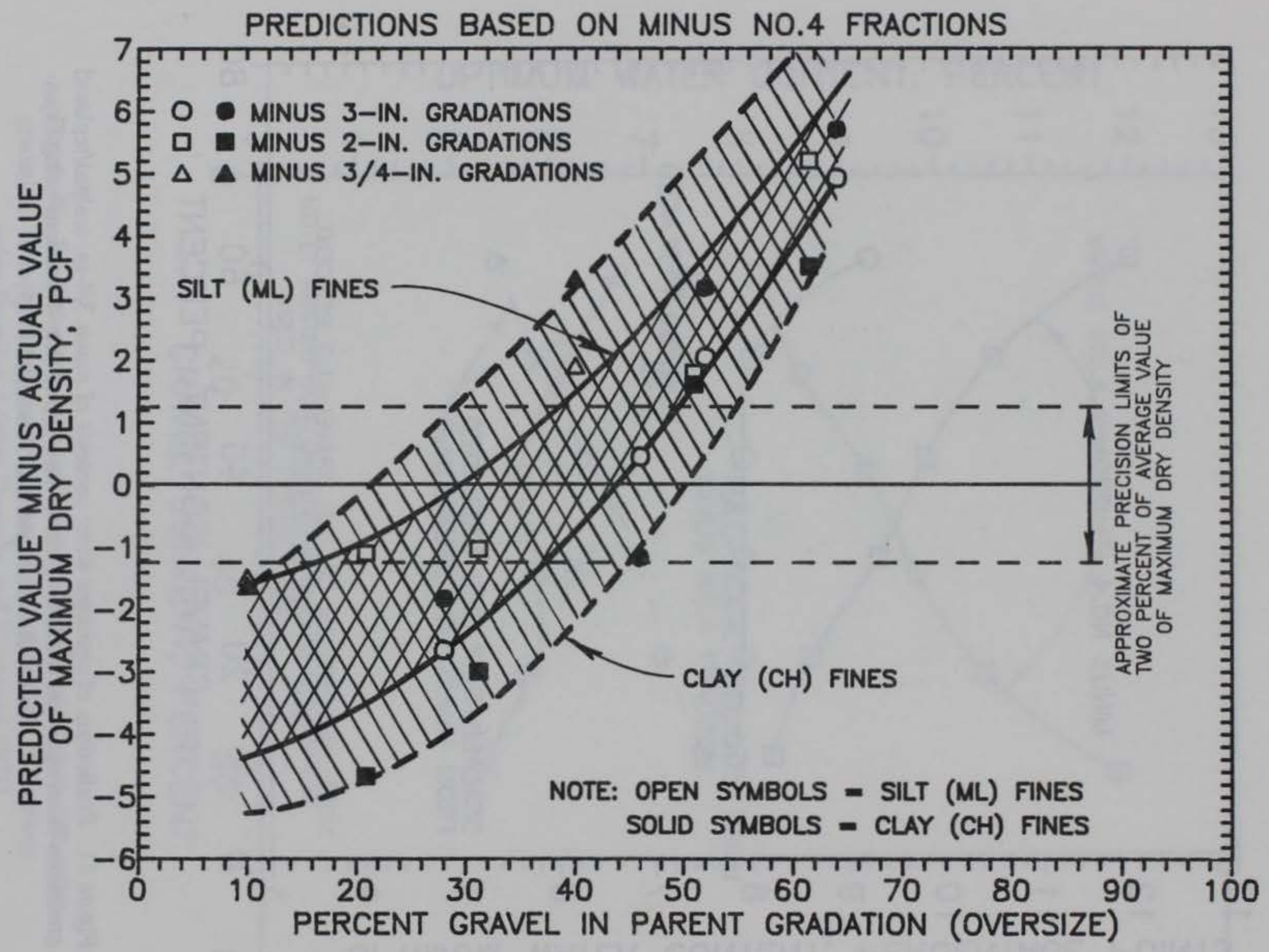

Figure 42. Summary plot for predictions of maximum dry densities of gradations of various maximum particle sizes from maximum dry densities of their minus No. 4 fractions (after Torrey and Donaghe 1991) 


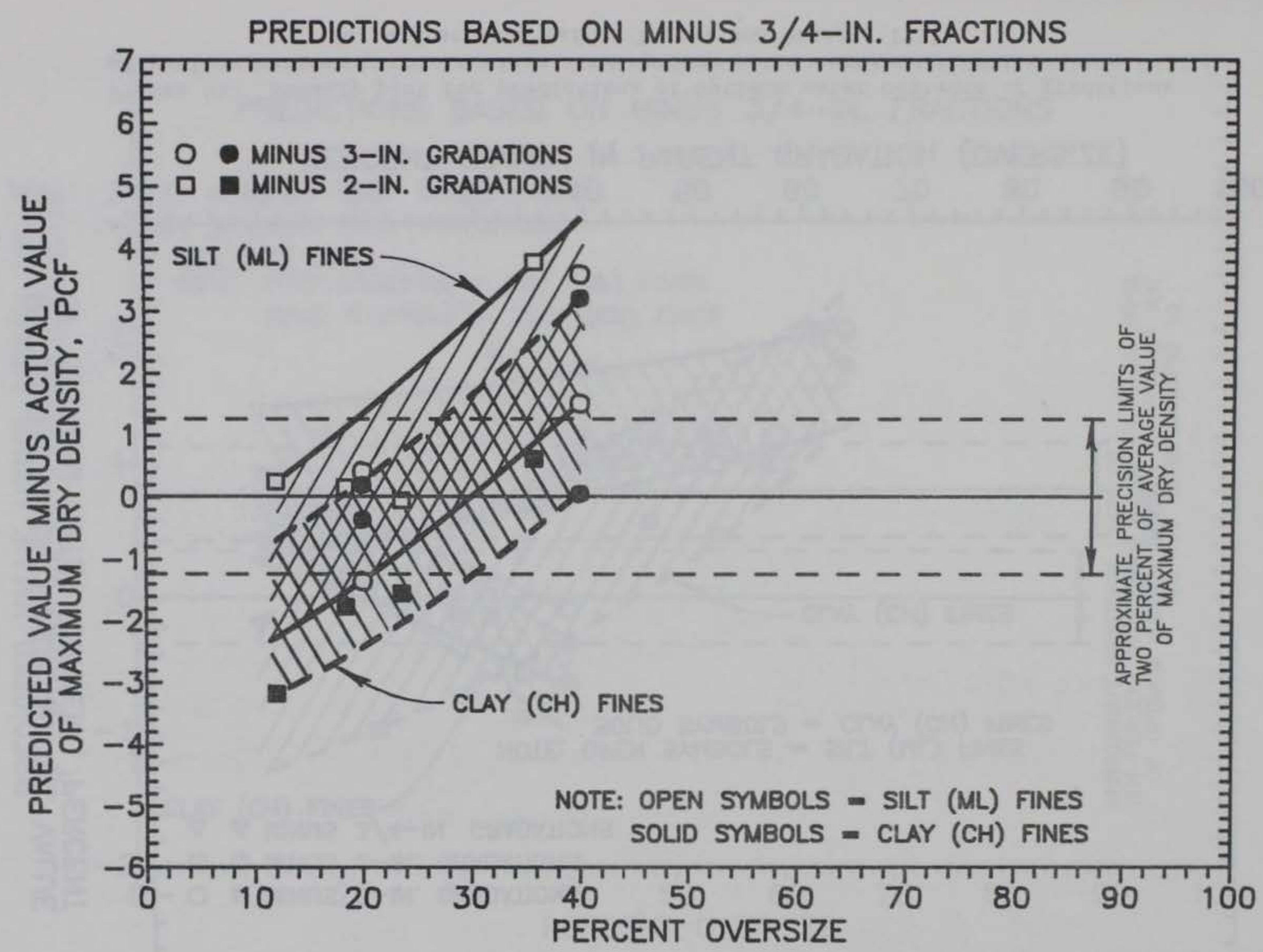

Figure 43. Summary plot for predictions of maximum dry densities of gradations of various maximum particle sizes from maximum dry densities of their minus 3/4-in. fractions (after Torrey and Donaghe 1991) 


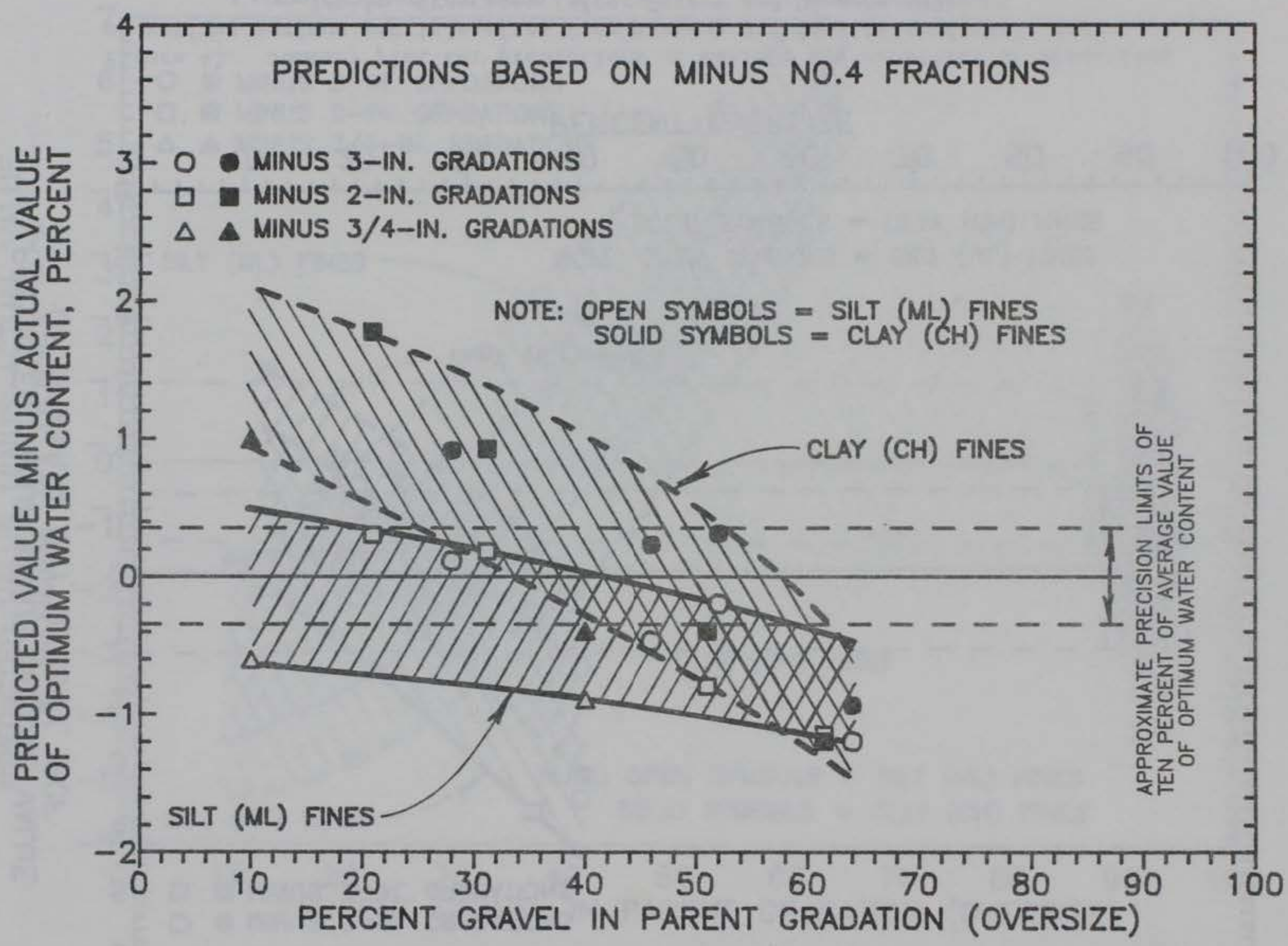

Figure 44. Summary plot for predictions of optimum water contents of gradations of various maximum particle sizes from optimum water contents of their minus

No. 4 fractions (after Torrey and Donaghe 1991) 


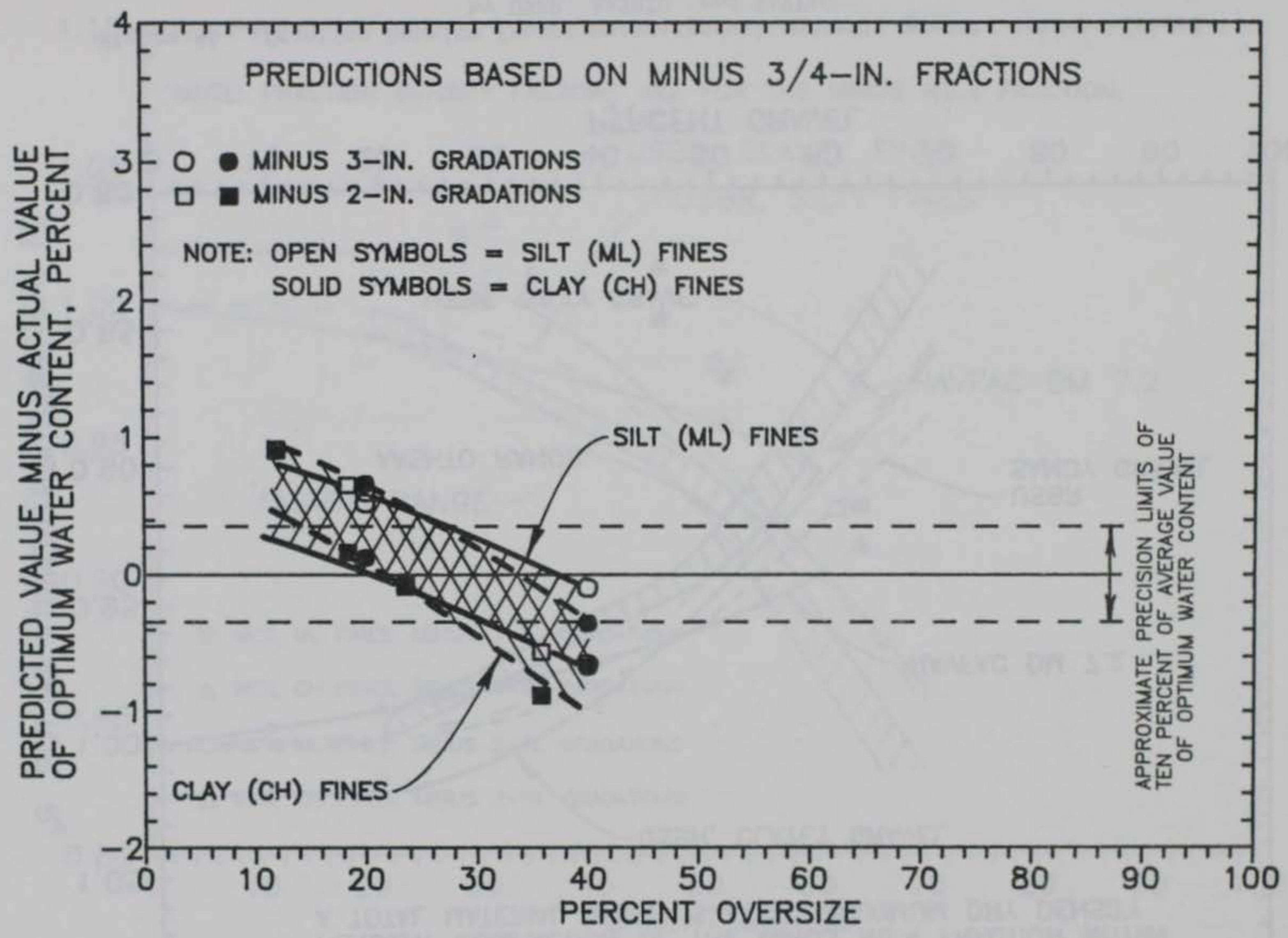

Figure 45. Summary plot for predictions of optimum water content of gradations of various maximum particle sizes from optimum water contents of their minus 3/4-in. fractions (after Torrey and Donaghe 1991) 


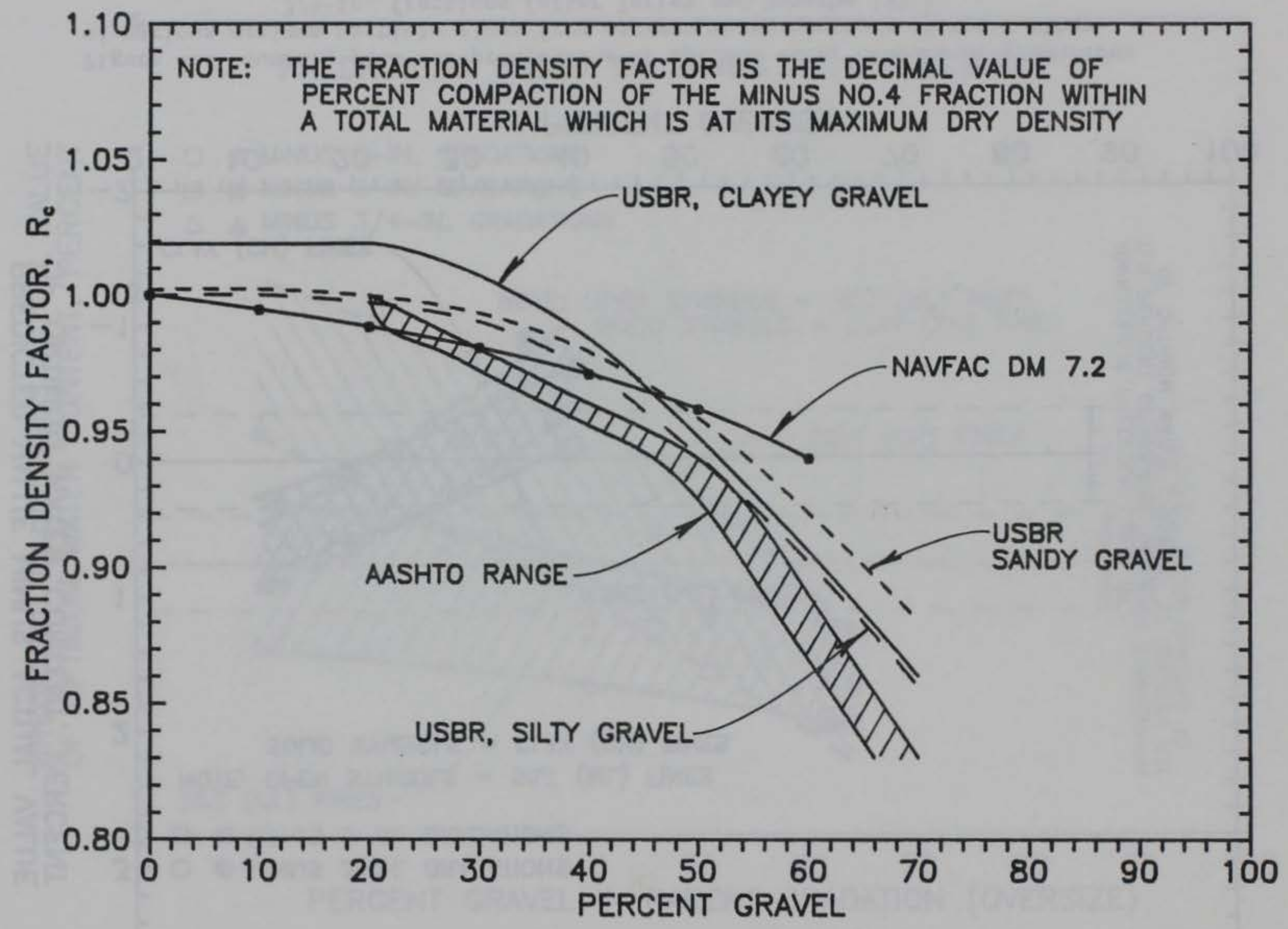

Figure 46. Fraction Density Factor versus gravel content, generic curves offered by USBR, AASHTO, and NAVFAC 


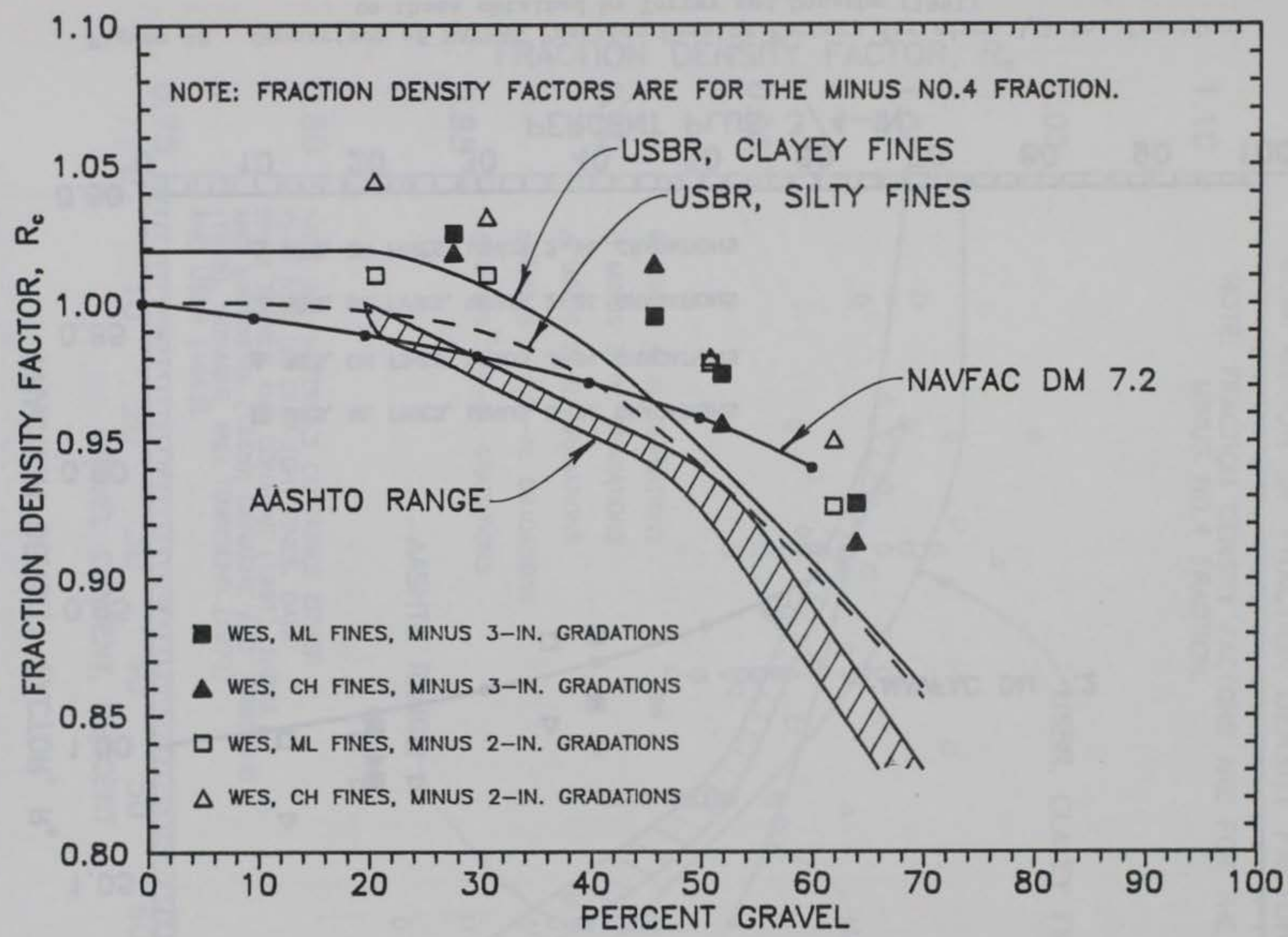

Figure 47. Comparison of USBR, AASHTO, NAVFAC Fraction Density Factors for minus No. 4 fractions to those obtained by Torrey and Donaghe (1991) 


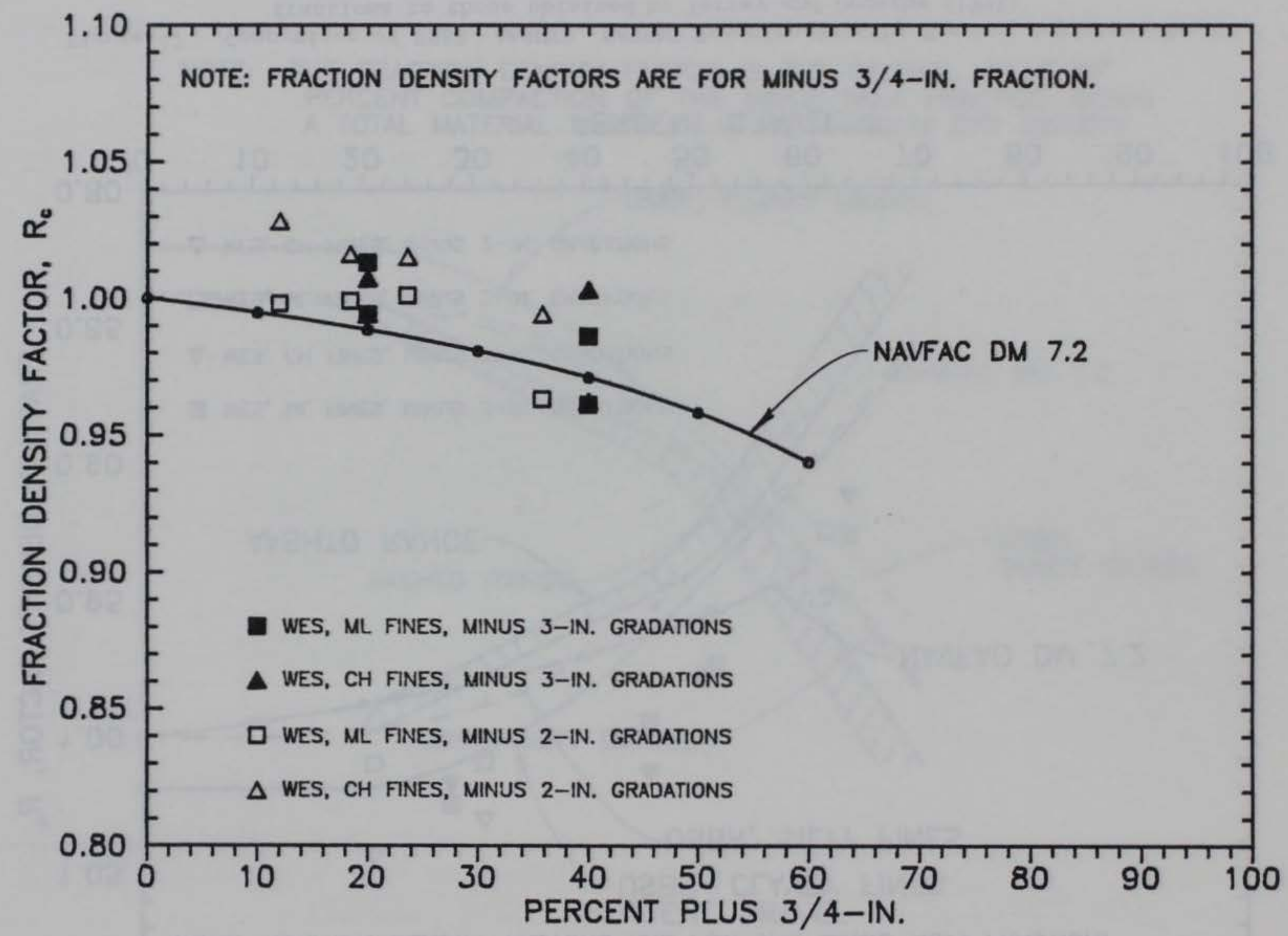

Figure 48. Comparison of NAVFAC Fraction Density Factors for minus 3/4-in. fractions to those obtained by Torrey and Donaghe (1991) 


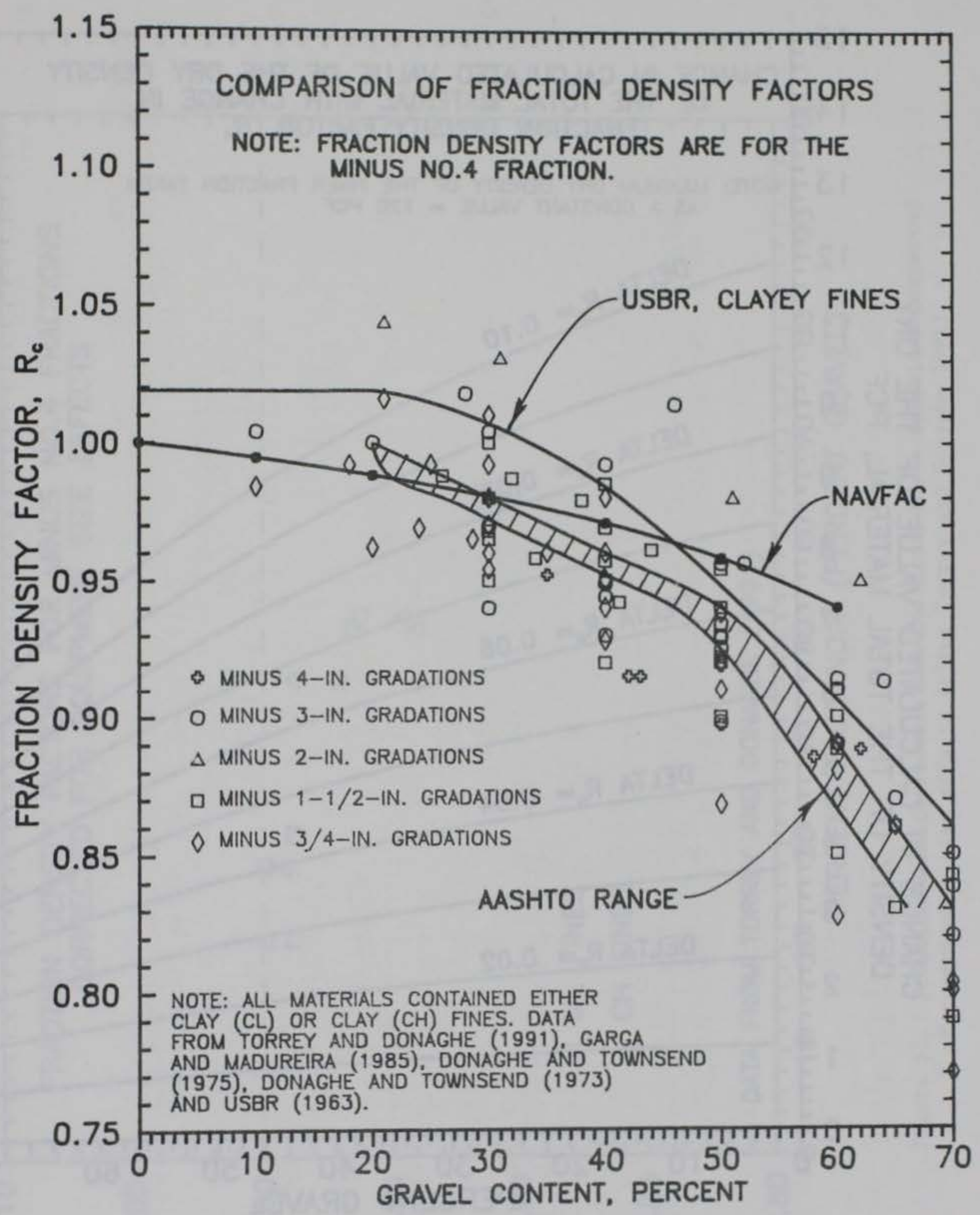

Figure 49. Range in Fraction Density Factors for minus No. 4 fractions obtained among various investigators 


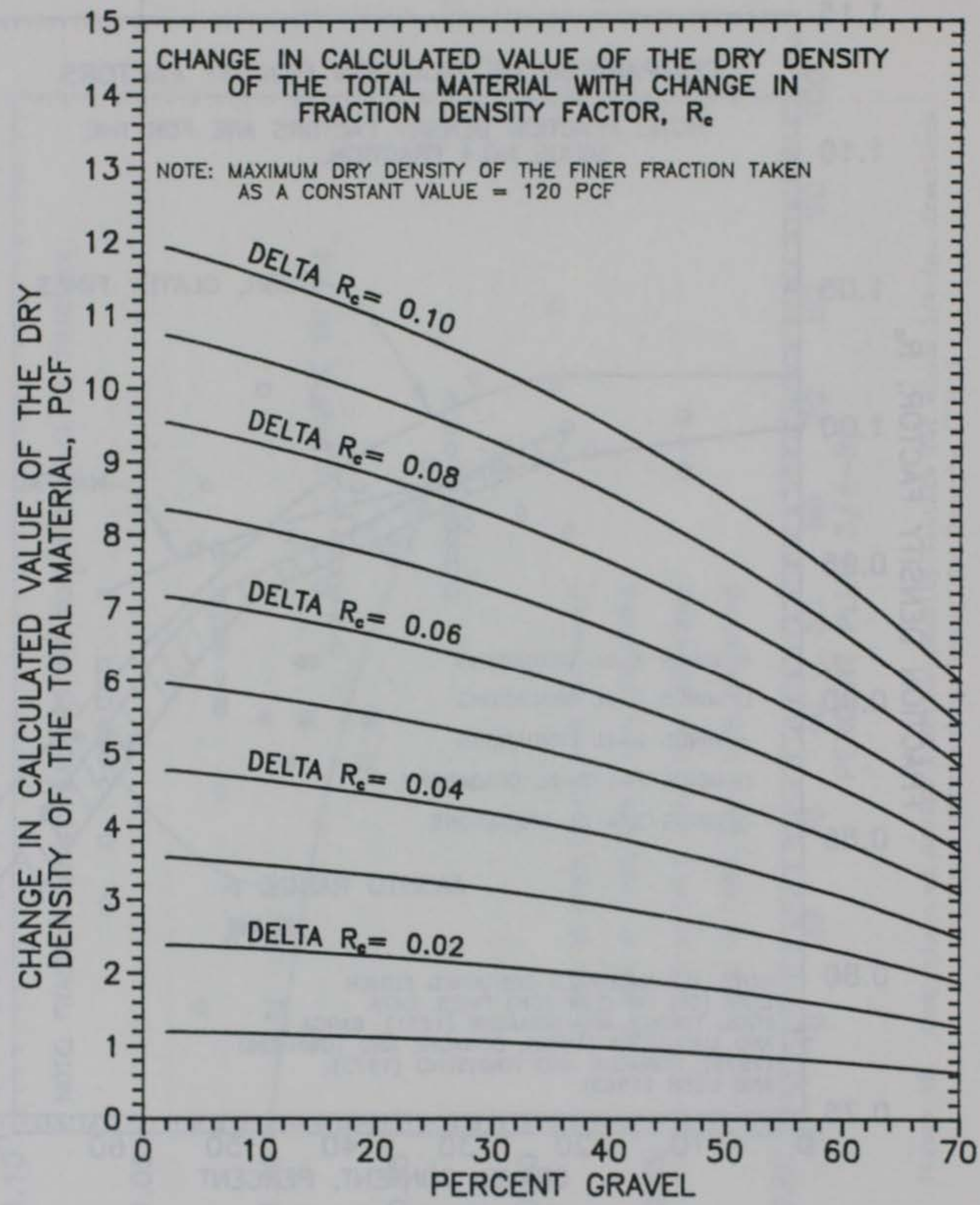

Figure 50. Change in calculated value of maximum dry density of the total material with change in Fraction Density Factor for the minus No. 4 fraction 


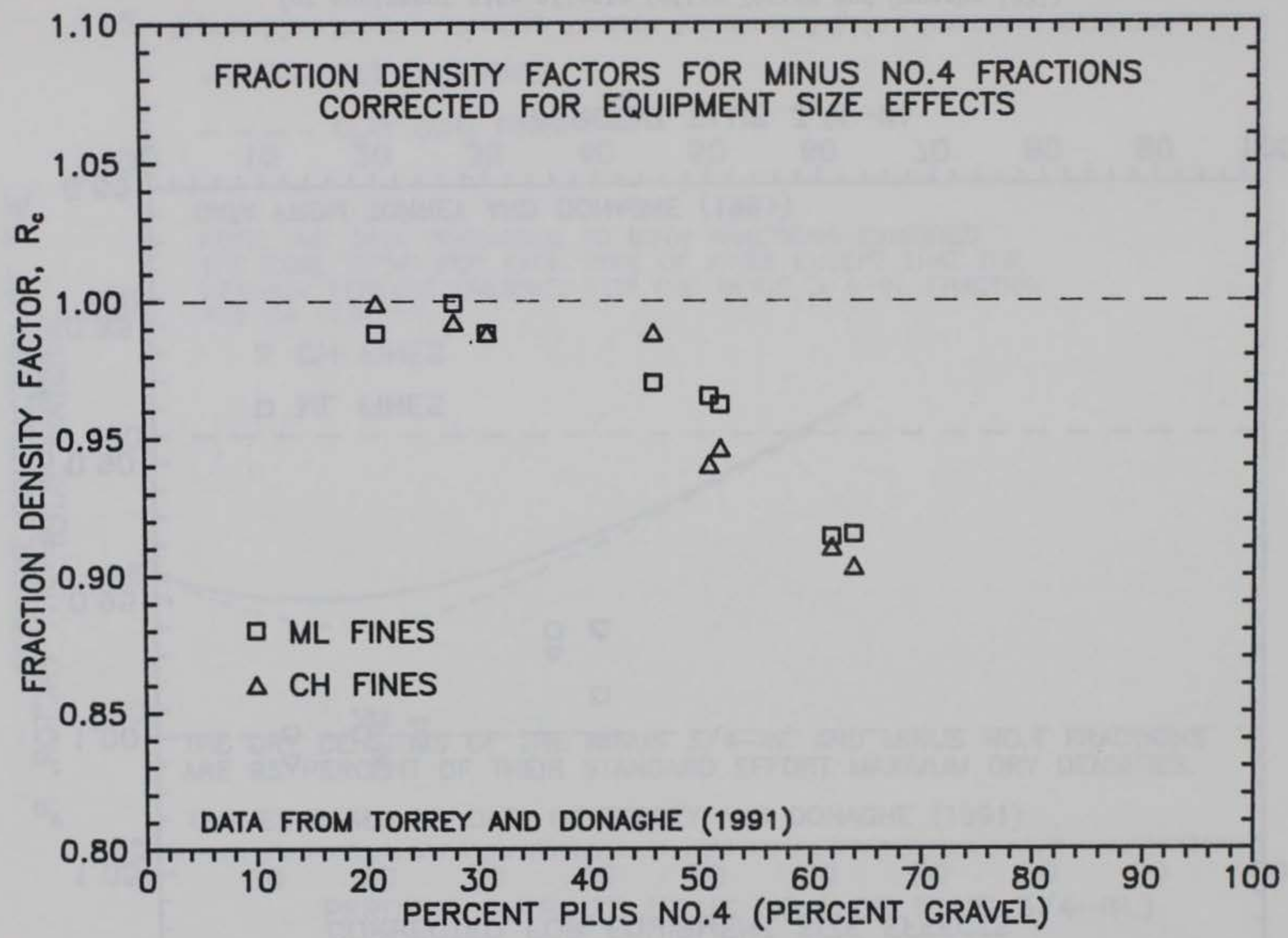

Figure 51. Fraction Density Factors for the minus No. 4 fractions corrected for equipment size effects (after Torrey and Donaghe 1991) 


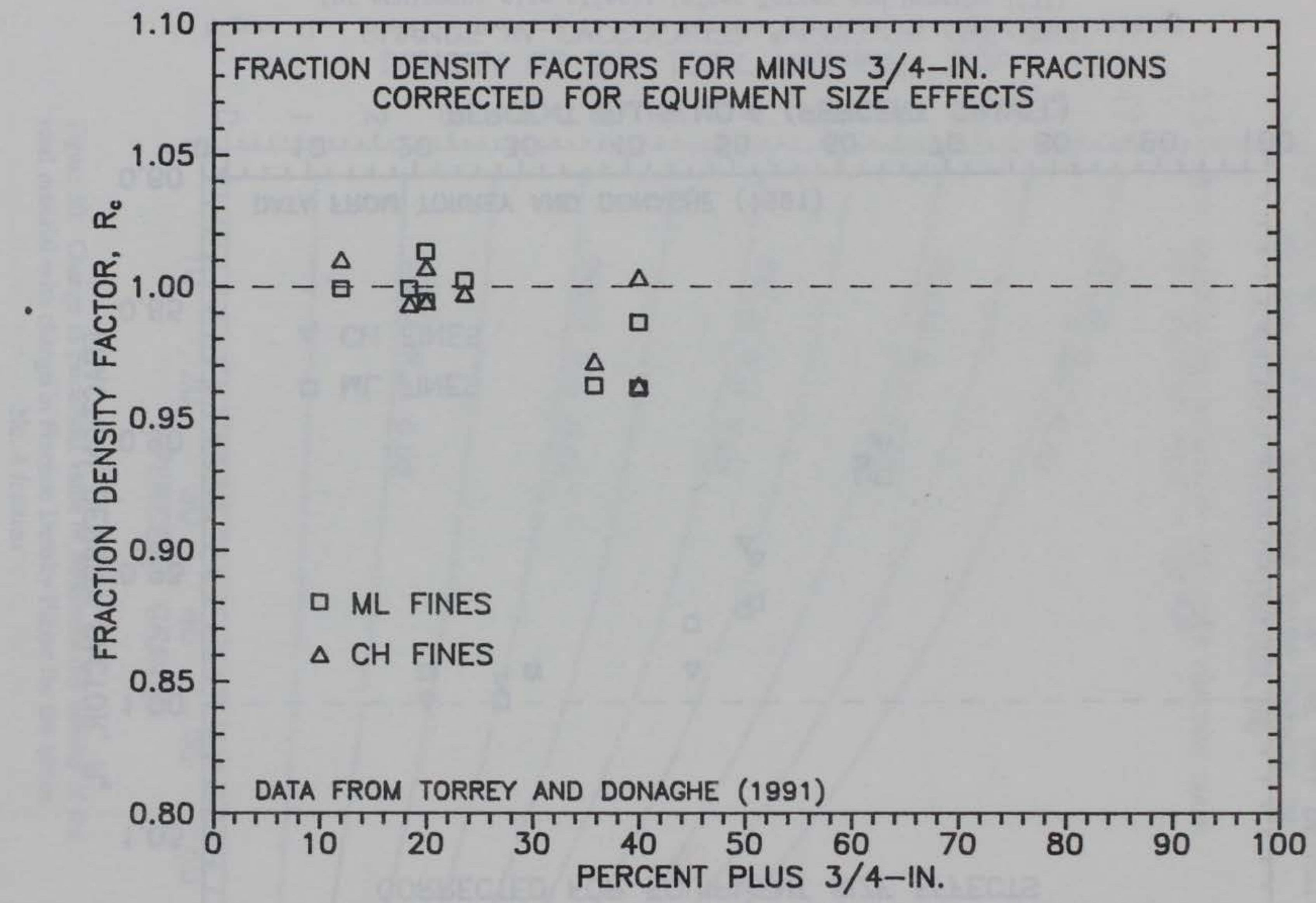

Figure 52. Fraction Density Factors for minus 3/4-in. fractions corrected for equipment size effects (after Torrey and Donaghe 1991) 


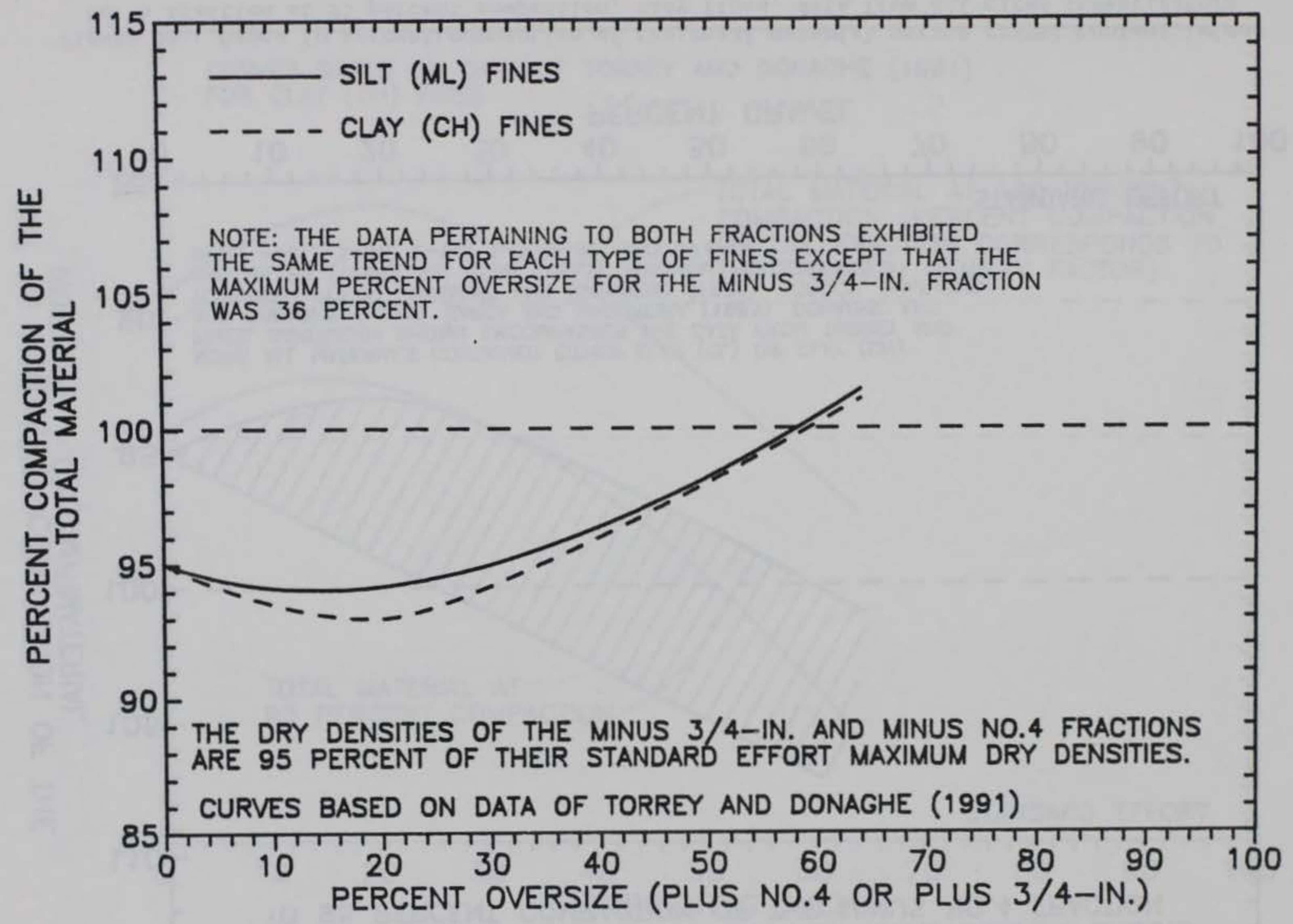

Figure 53. Percent compaction of the total material versus percent oversize, minus 3/4-in. and minus No. 4 fractions at 95 percent compaction (data from Torrey and Donaghe 1991) 


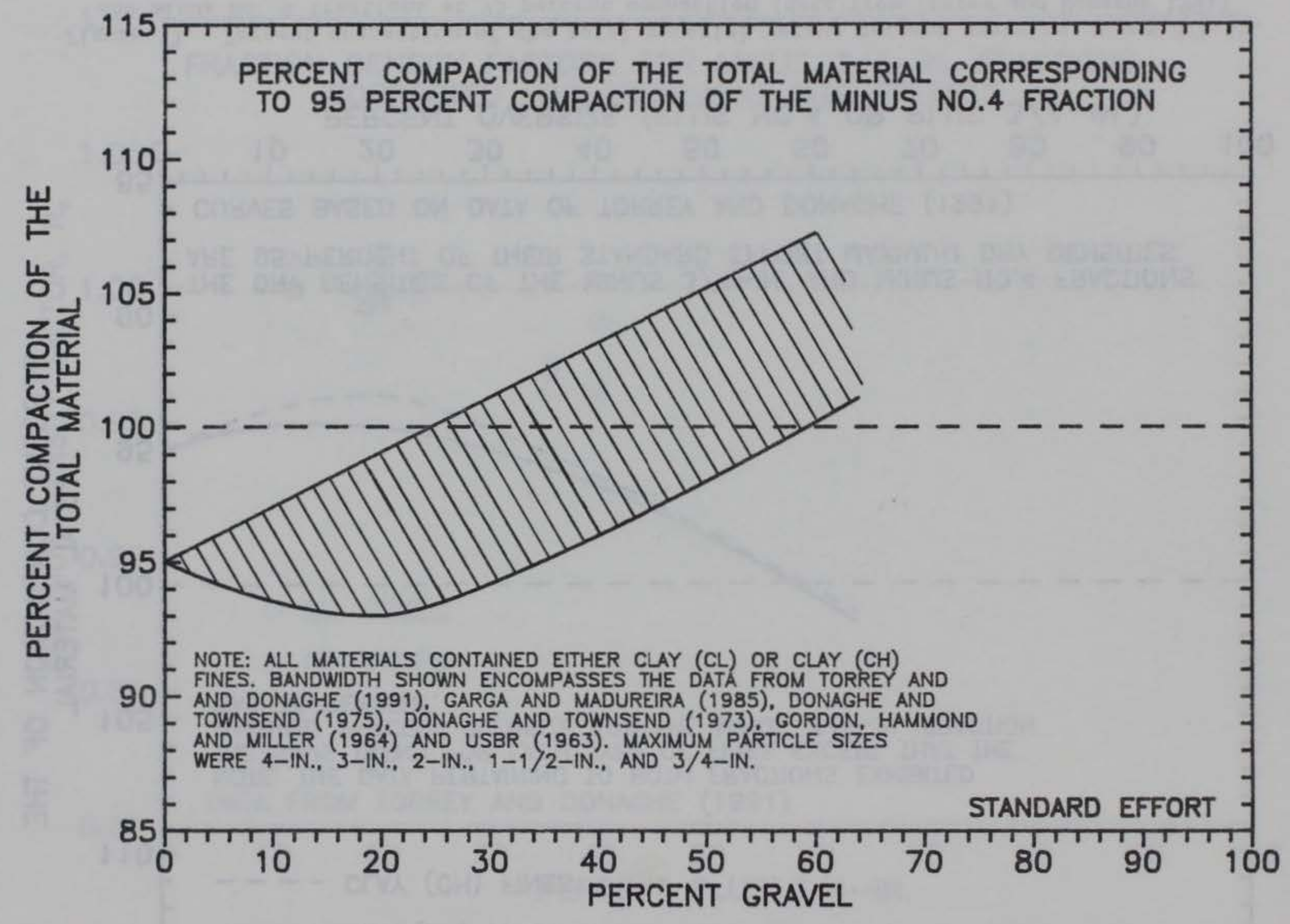

Figure 54. Range in percent compaction of the total material versus gravel content, minus No. 4 fraction at 95 percent compaction, clay fines, data from all cited investigators 


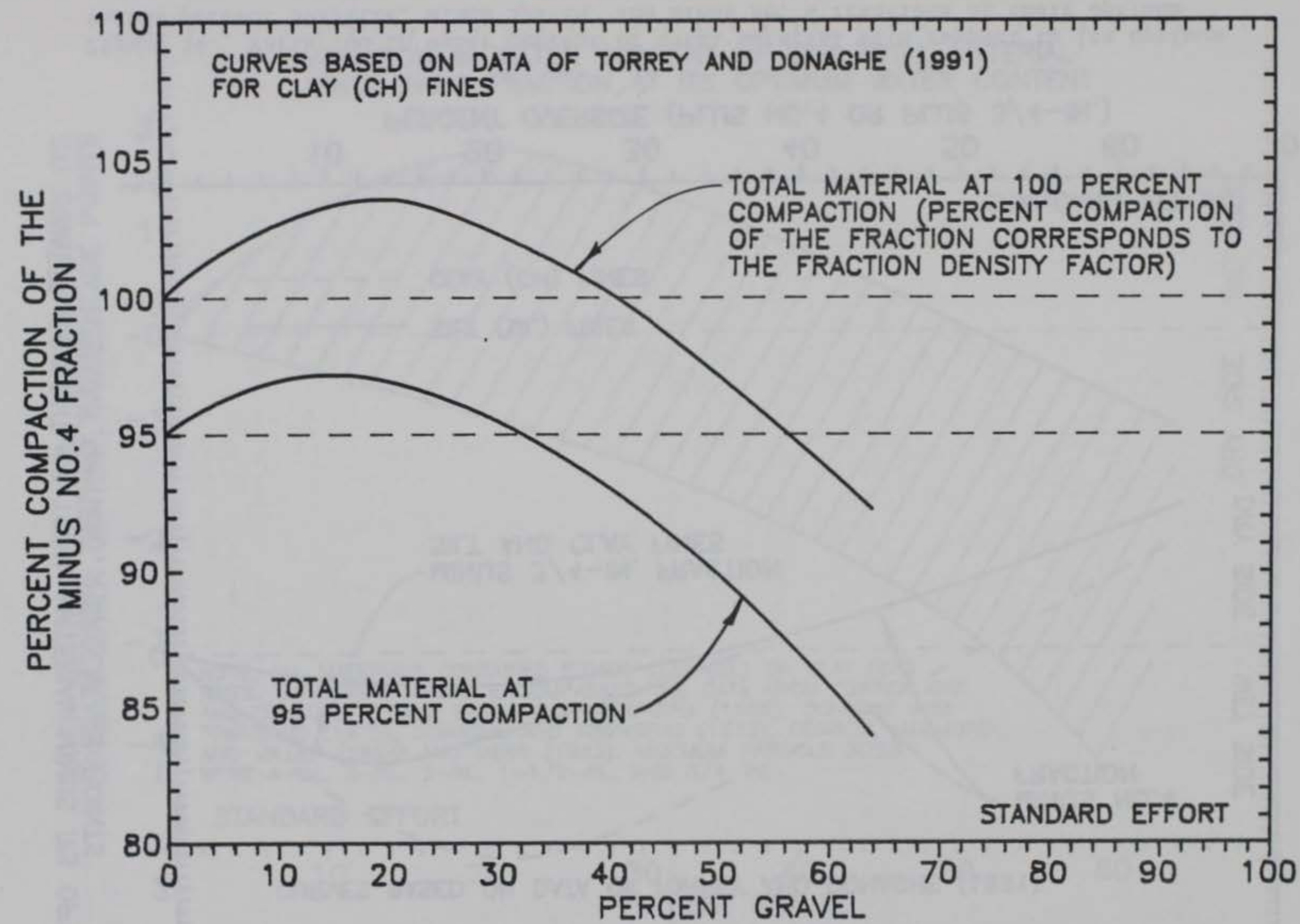

Figure 55. Percent compaction of the minus No. 4 fraction versus gravel content of the total material, total material at 100 and 95 percent compaction, clay (CH) fines, data from Torrey and Donaghe (1991) 


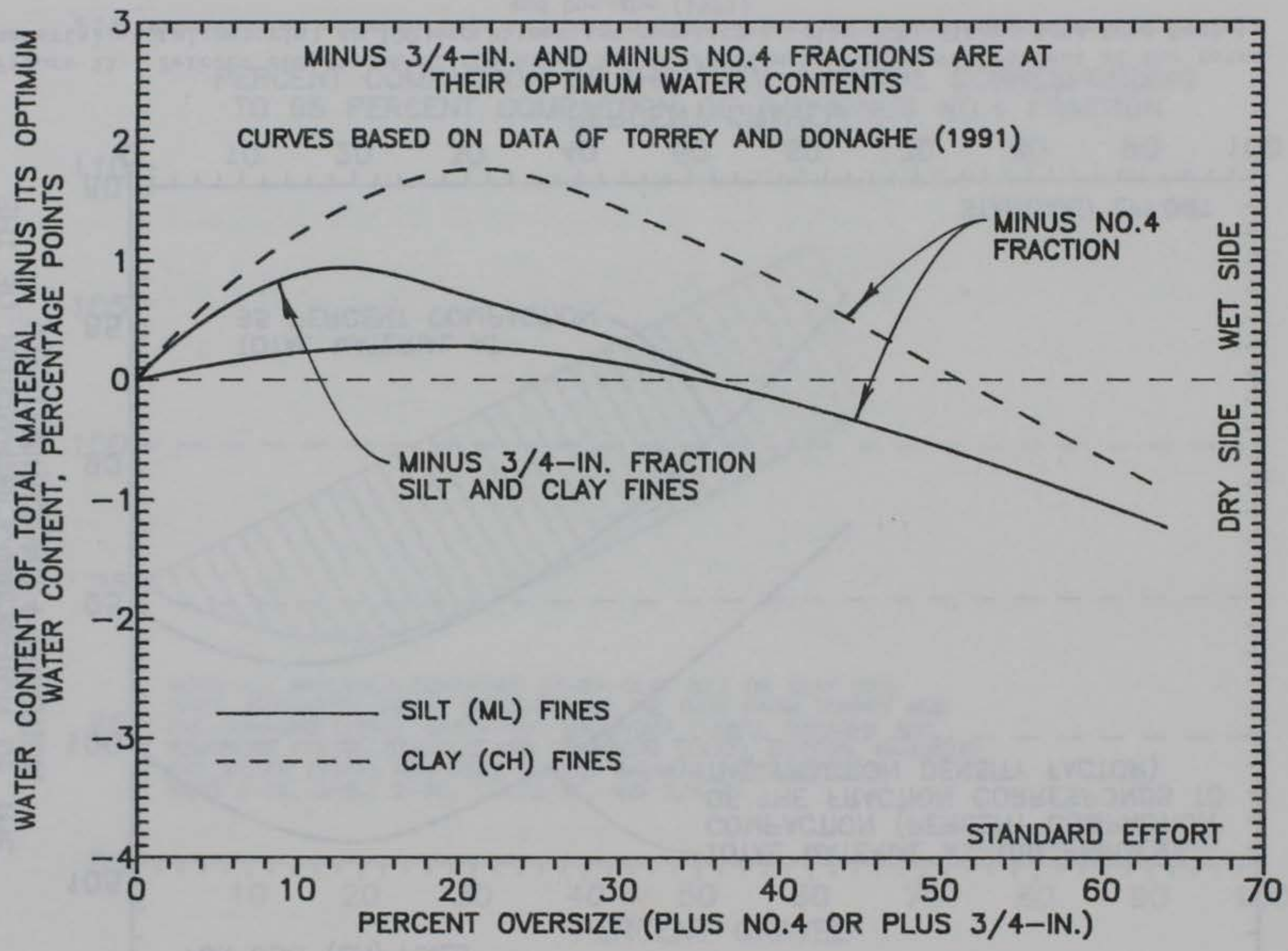

Figure 56. Variation in water content of total material with respect to its optimum versus percent oversize, minus $3 / 4-$ in. and minus No. 4 fractions at their optimum water content, data from Torrey and Donaghe (1991) 


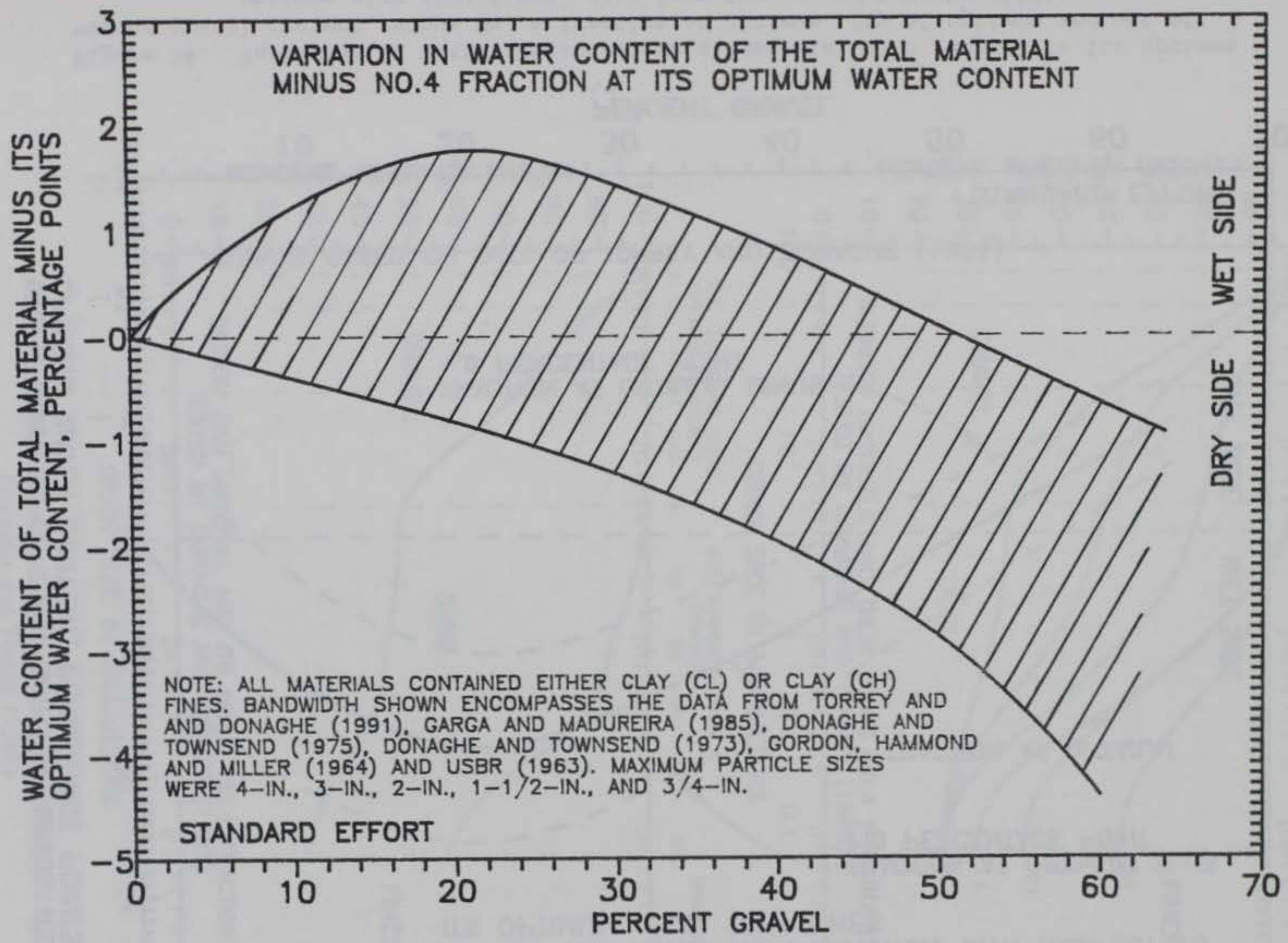

Figure 57. Range in water content of total material with respect to its optimum versus gravel content, minus No. 4 fraction at its optimum water content, clay fines, all cited investigators 


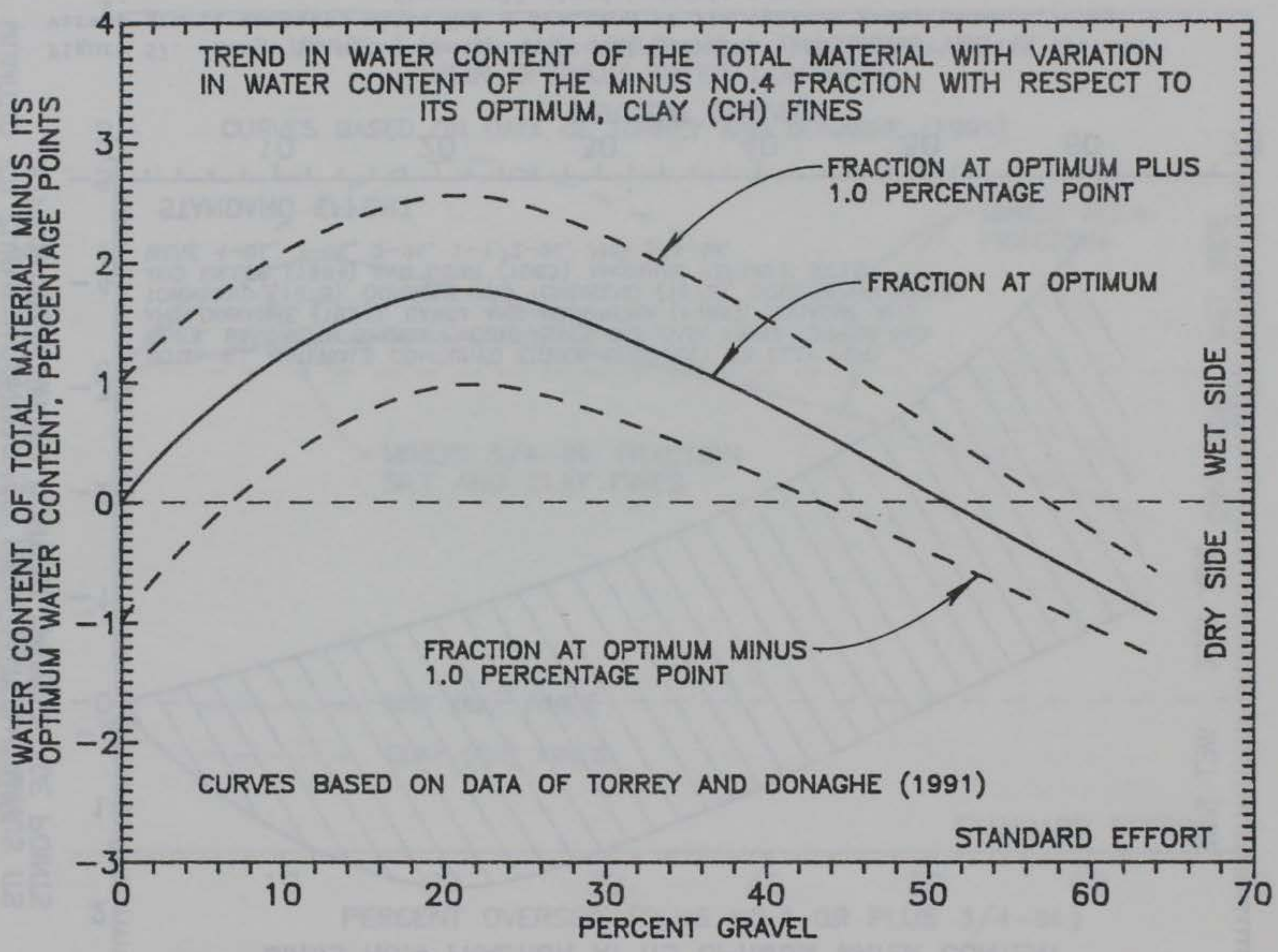

Figure 58. Variation of water content of total material with respect to its optimum versus gravel content, minus No. 4 fraction at optimum, dry of optimum and wet of optimum clay (CH) fines, data from Torrey and Donaghe (1991) 

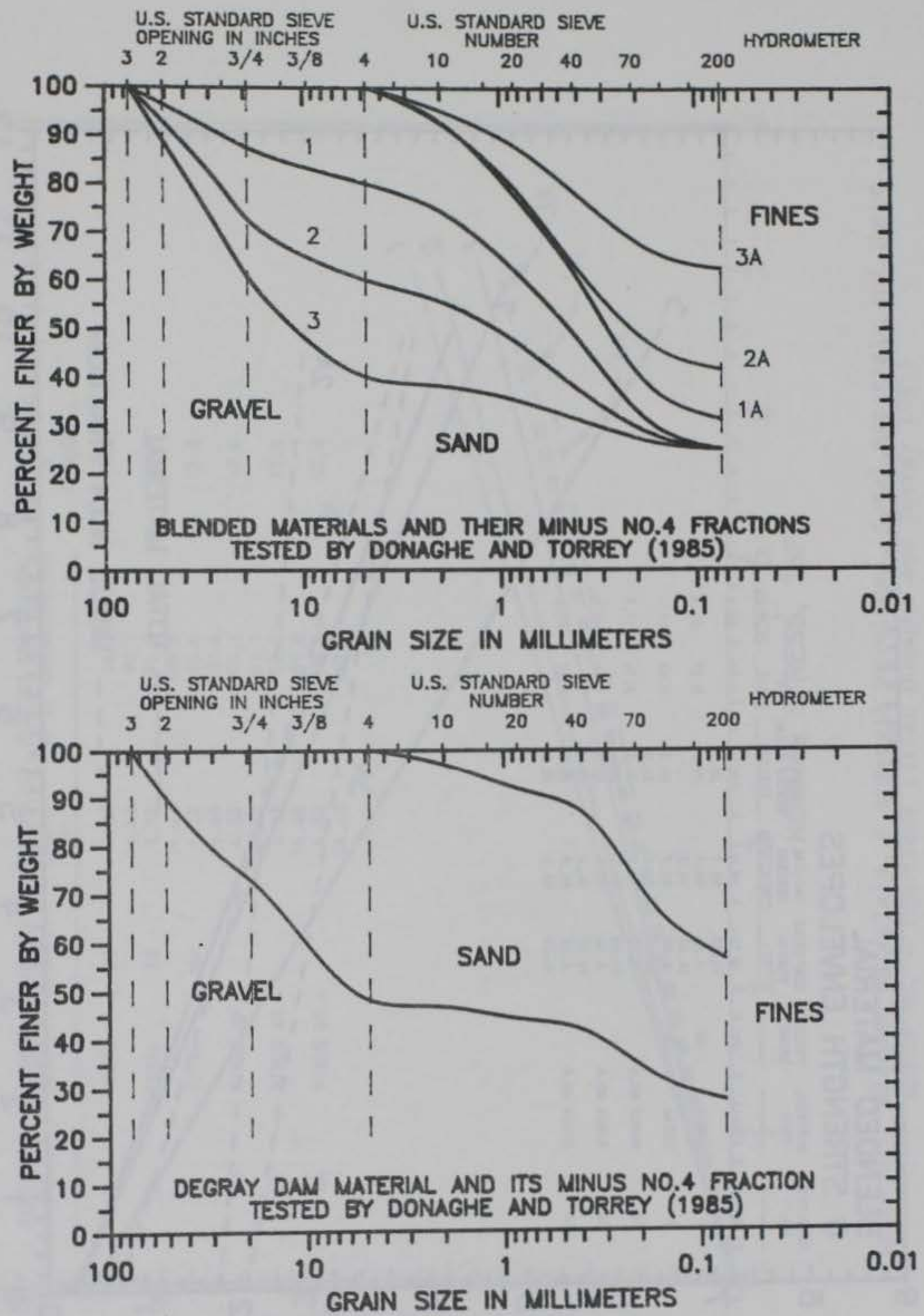

Figure 59. Blended and DeGray Dam materials tested by Donaghe and Torrey (1985) 


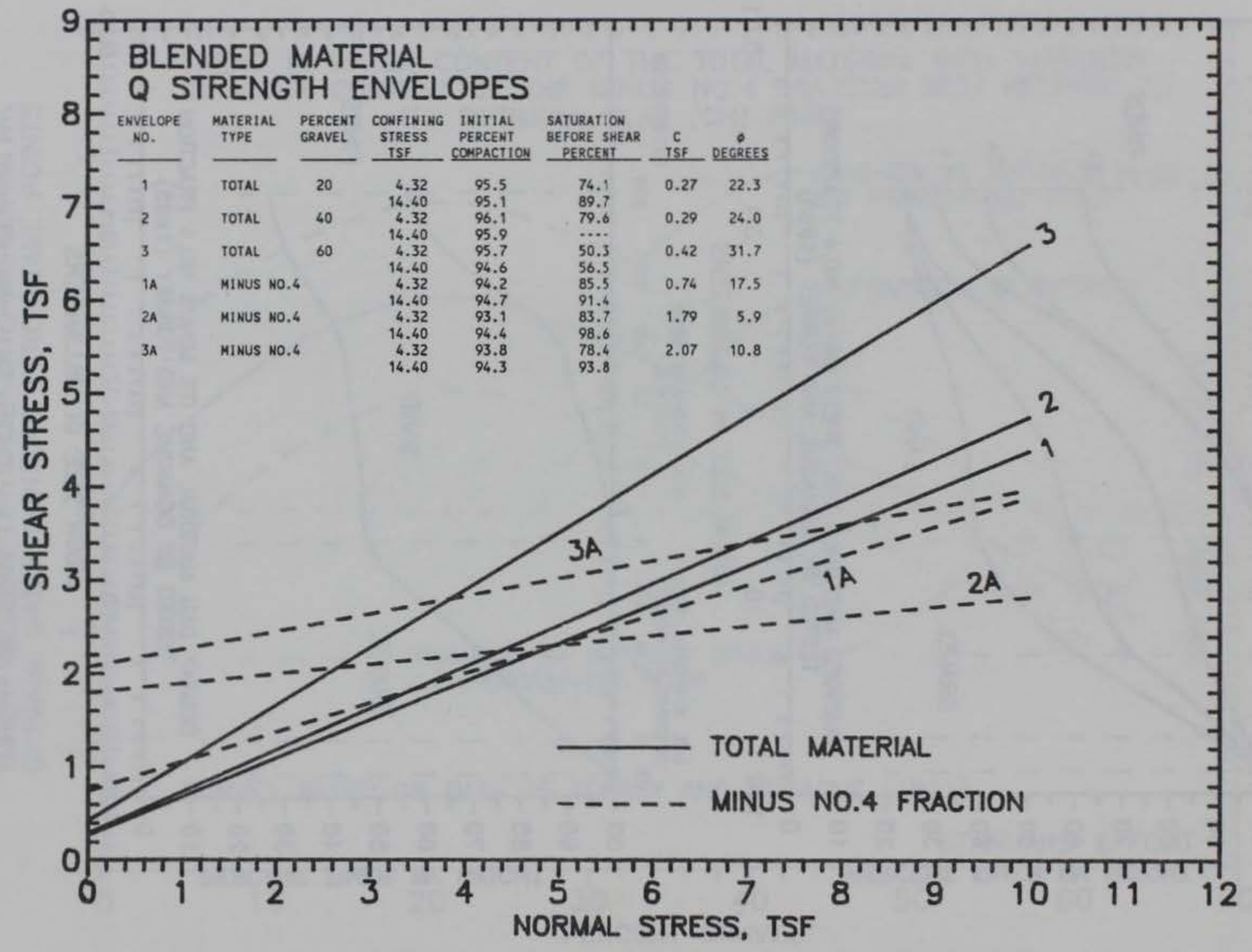

Figure 60. Blended materials, Q strength envelopes, total materials and their minus No. 4 fractions (after Donaghe and Torrey 1985) 


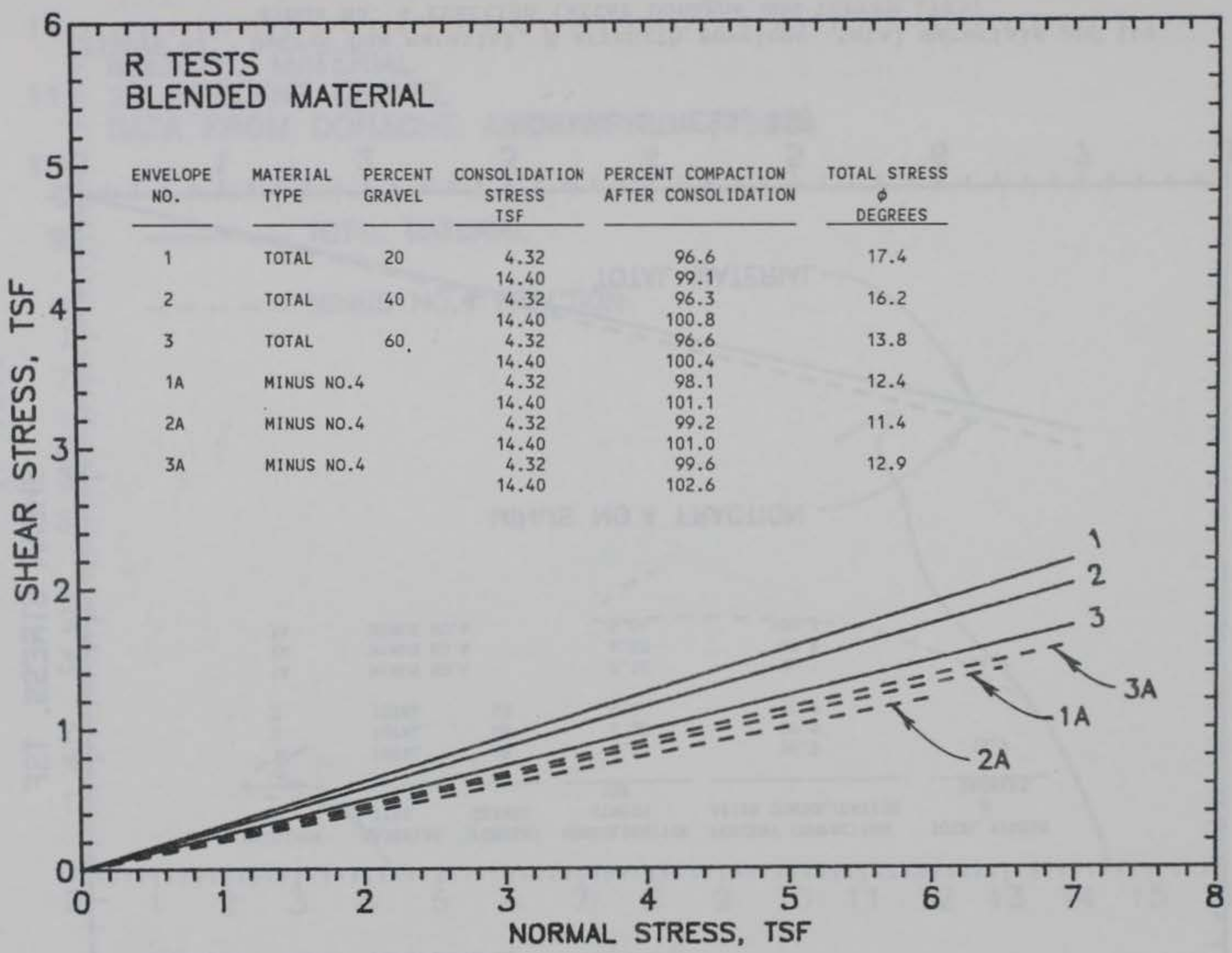

Figure 61. Blended materials, $\mathrm{R}$ strength envelopes, total materials and their minus No. 4 fractions (after Donaghe and Torrey 1985) 


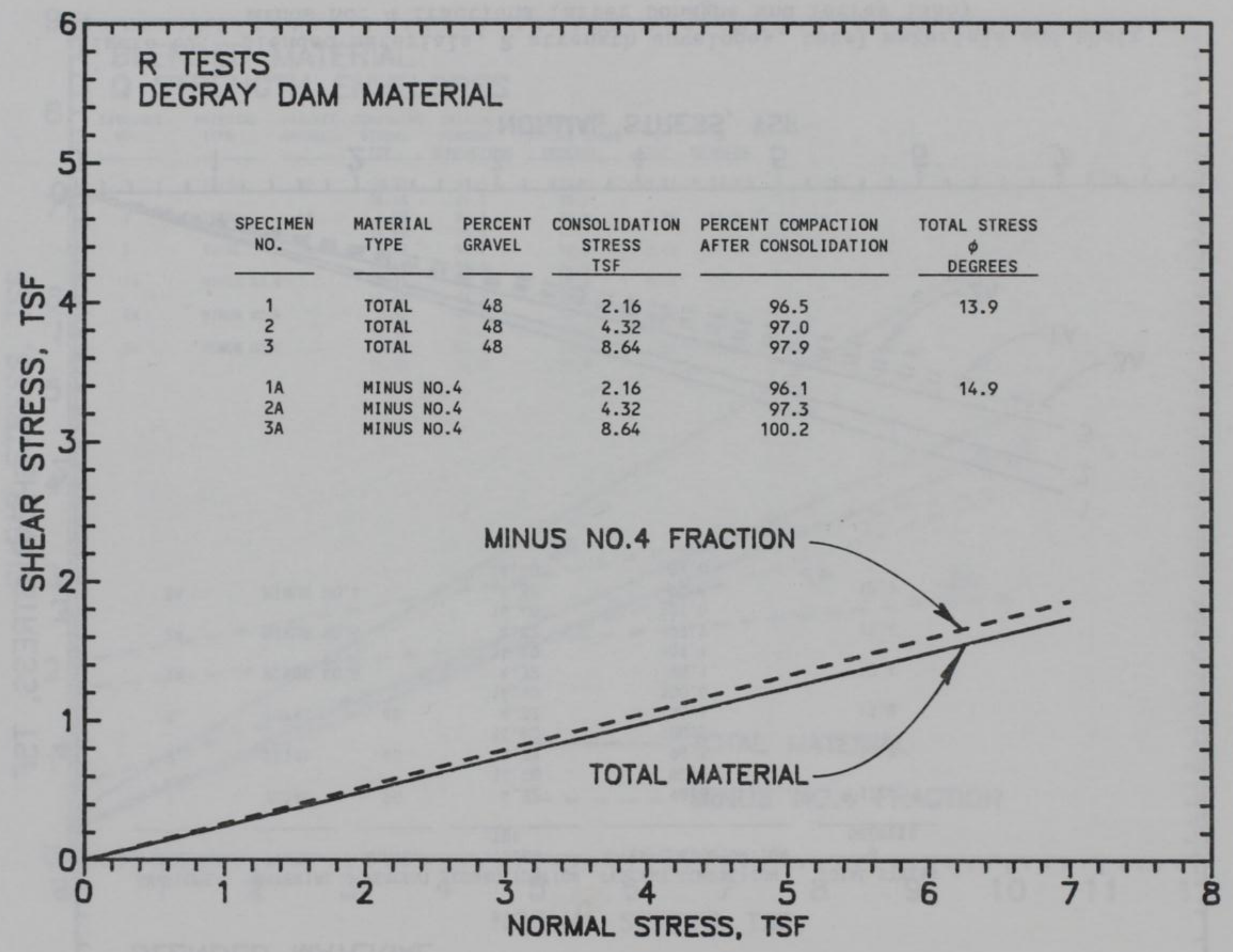

Figure 62. DeGray Dam material, $R$ strength envelope, total materials and its minus No. 4 fraction (after Donaghe and Torrey 1985) 


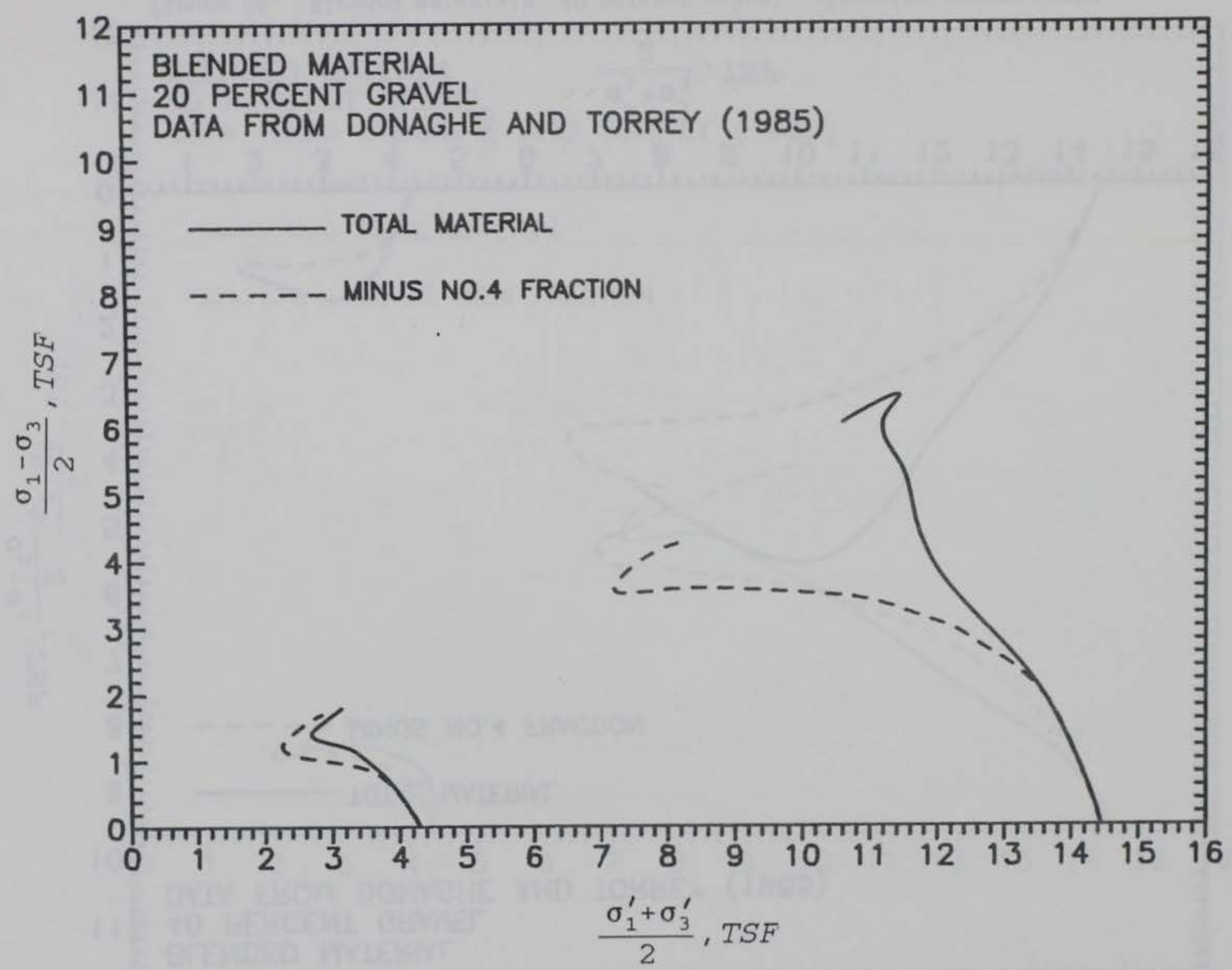

Figure 63. Blended materials, 20 percent gravel, effective stress paths (after Donaghe and Torrey 1985) 


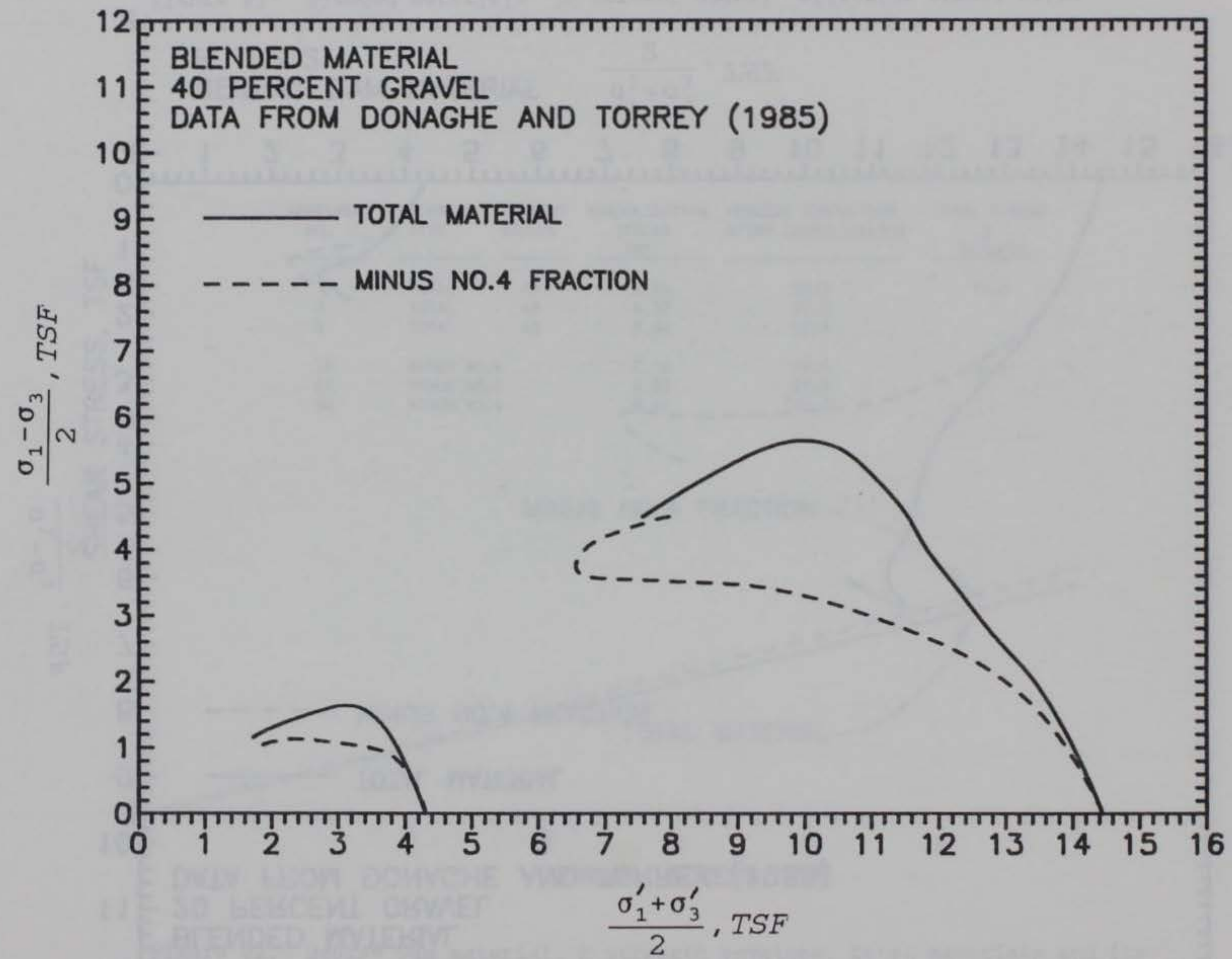

Figure 64. Blended materials, 40 percent gravel, effective stress paths (after Donaghe and Torrey 1985) 


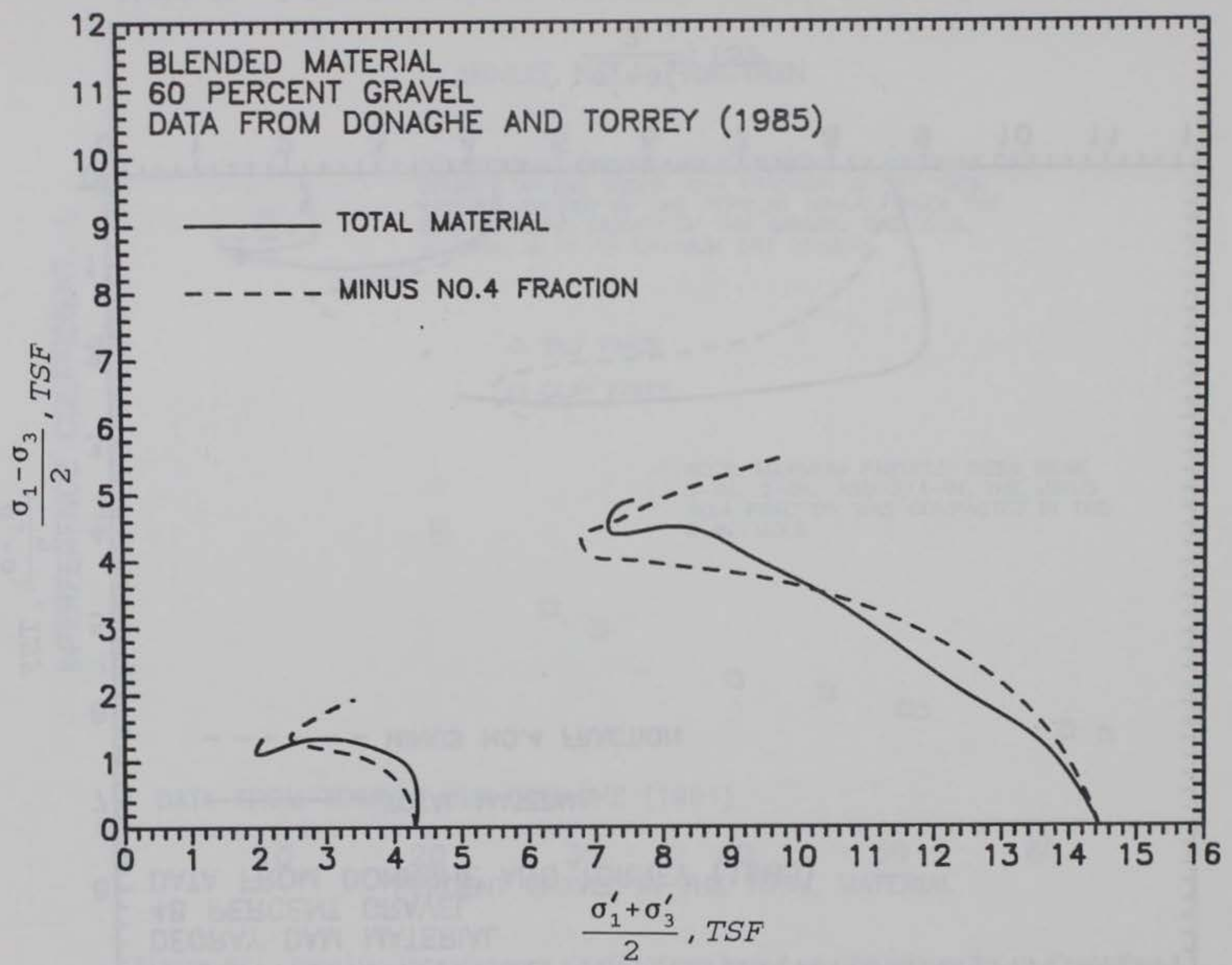

Figure 65. Blended materials, 60 percent gravel, effective stress paths (after Donaghe and Torrey 1985) 


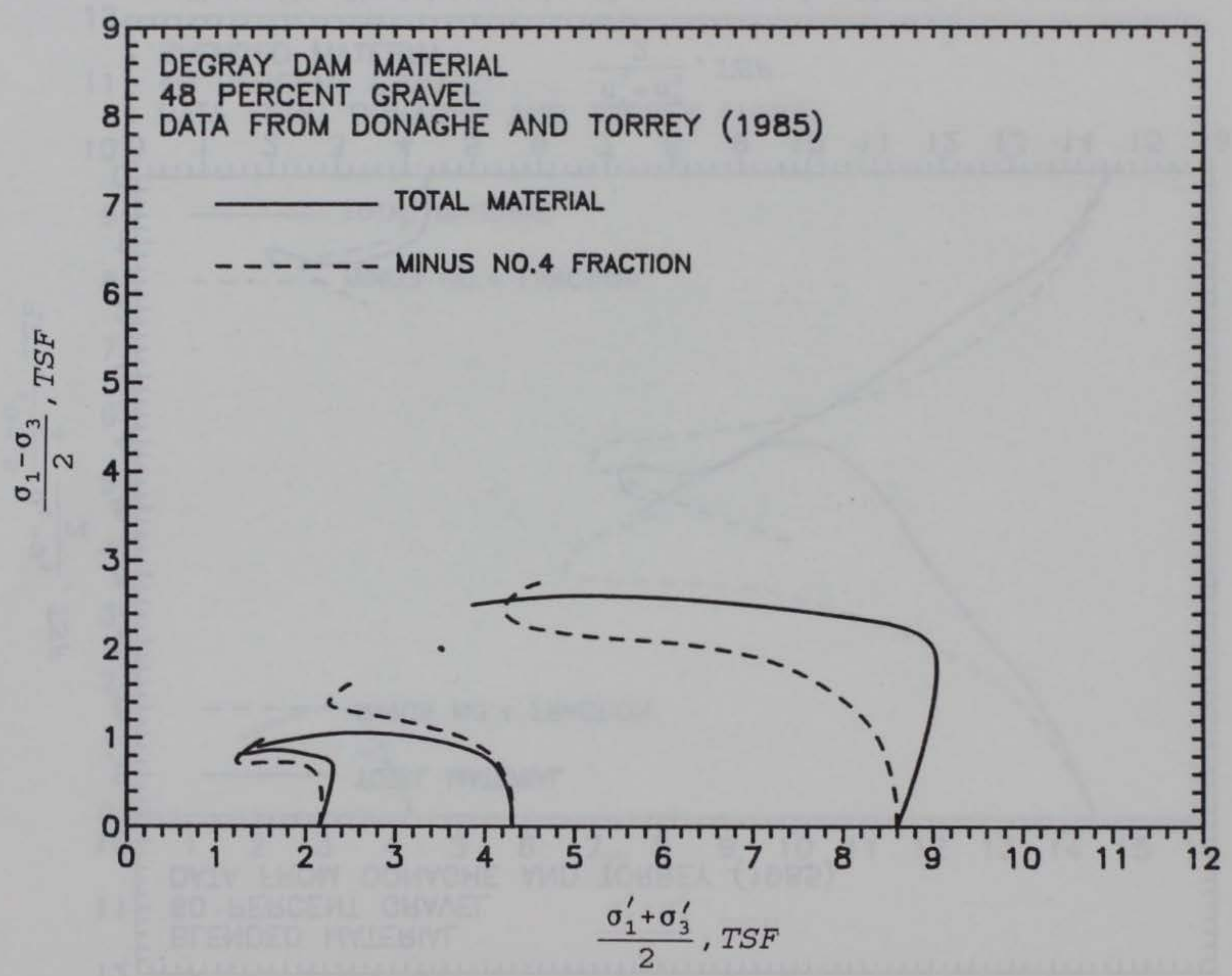

Figure 66. DeGray Dam material, 48 percent gravel, effective stress paths (after Donaghe and Torrey 1985) 


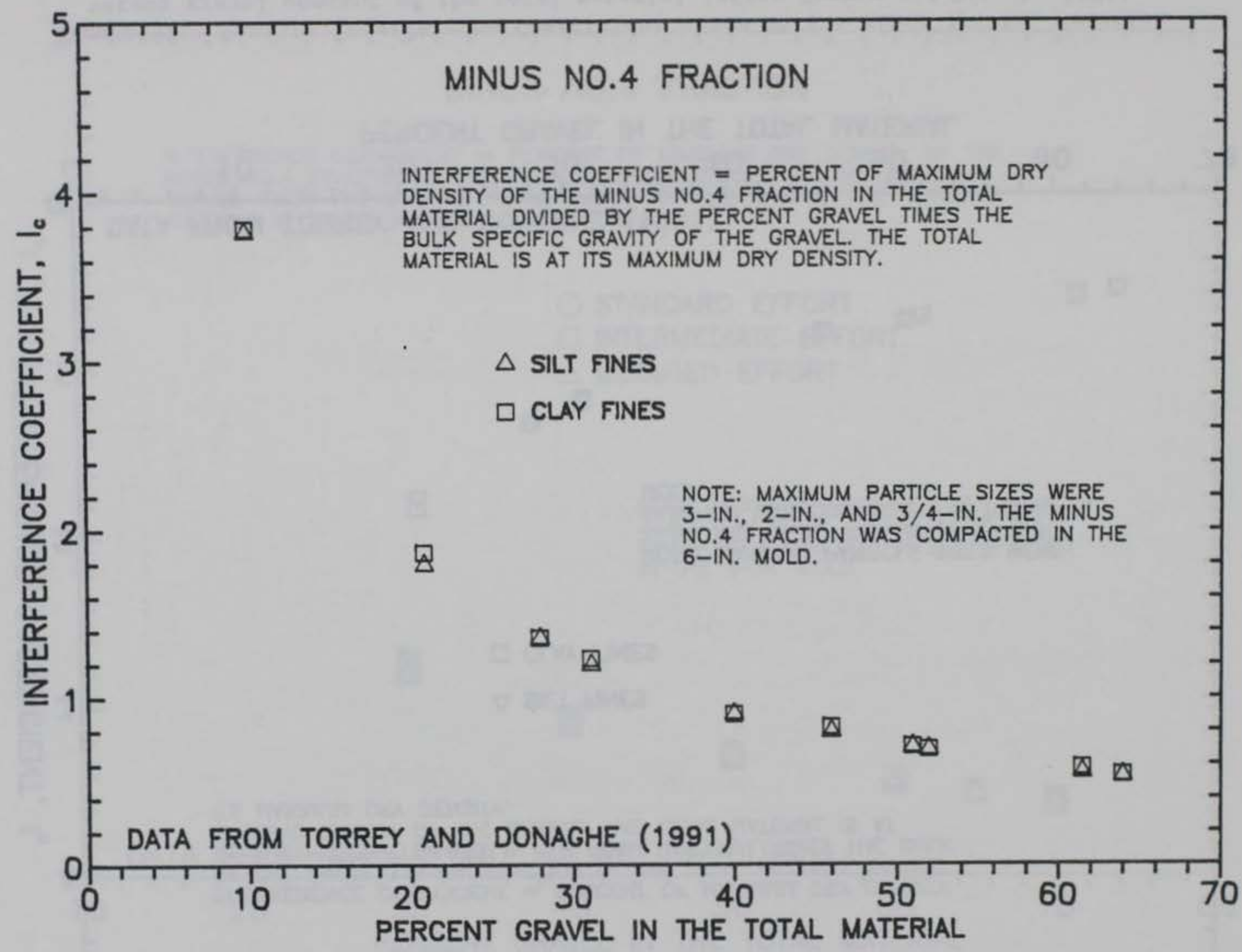

Figure 67. Density Interference Coefficient based on the minus No. 4 fraction versus gravel content of the total material (after Torrey and Donaghe 1991) 


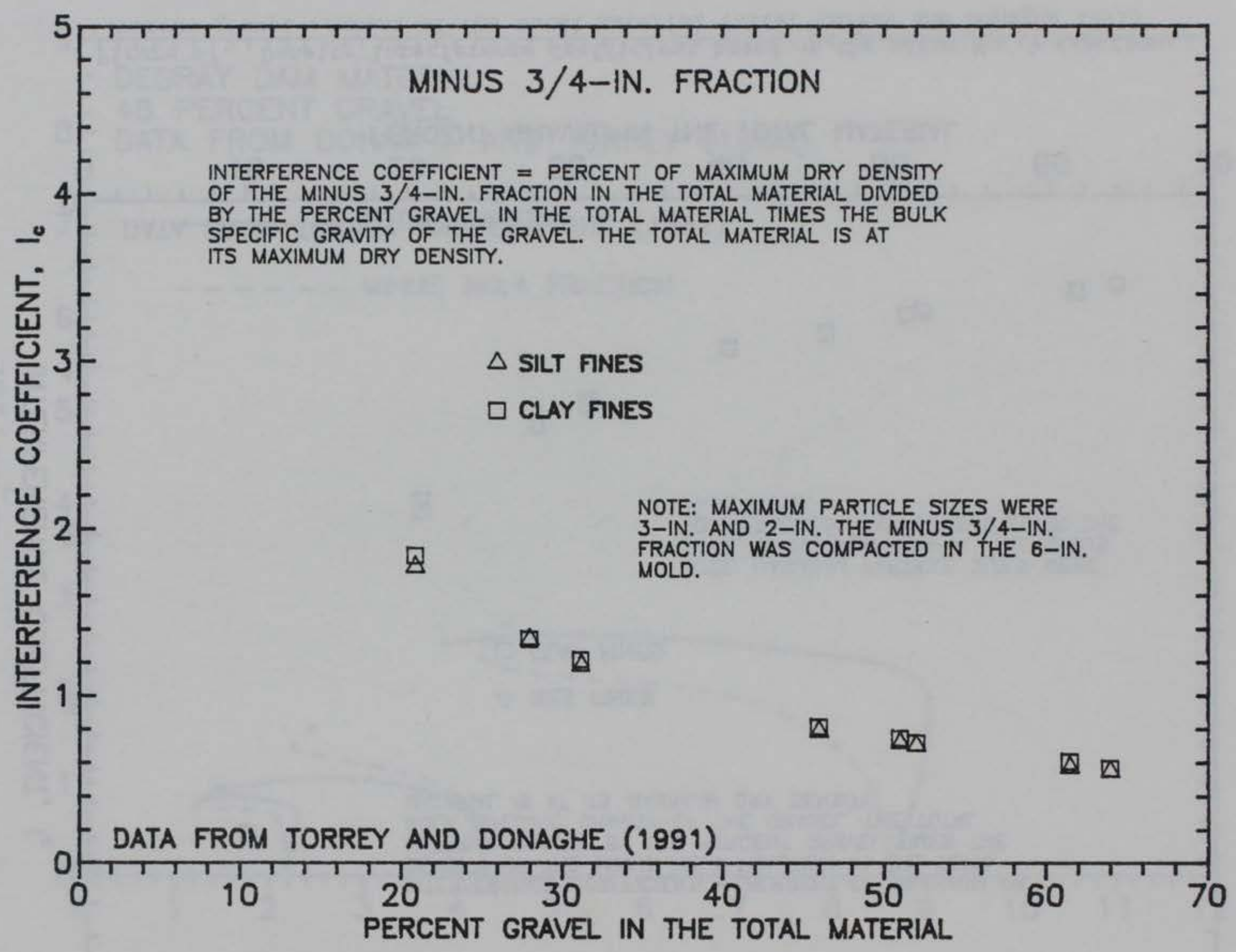

Figure 68. Density Interference Coefficient based on the minus 3/4-in. fraction versus gravel content of the total material (after Torrey and Donaghe 1991) 


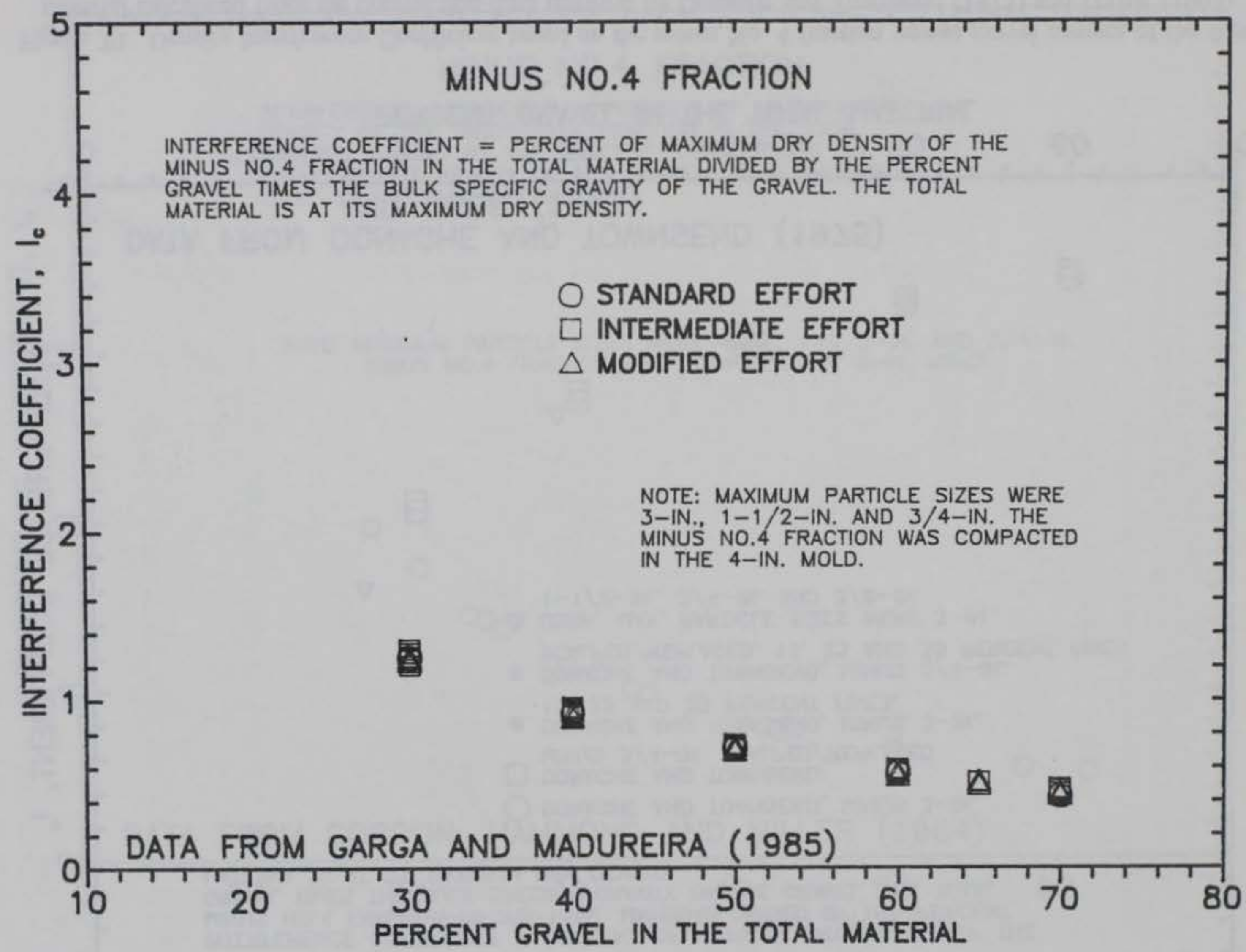

Figure 69. Density Interference Coefficient based on the minus No. 4 fraction versus gravel content of the total material calculated from the compaction data reported by Garga and Madureira (1985) 


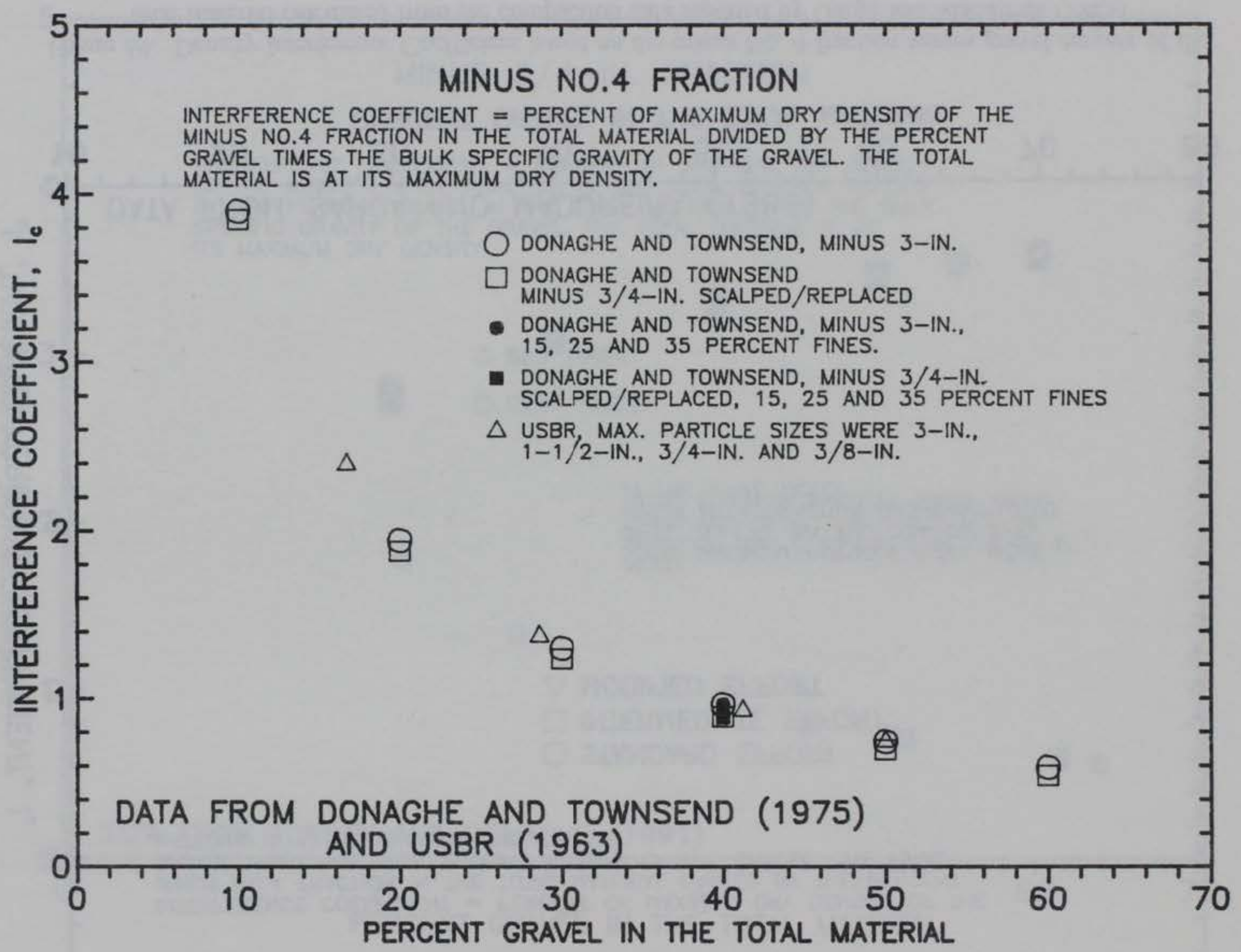

Figure 70. Density Interference Coefficient based on the minus No. 4 fraction versus gravel content of the total material calculated from the compaction data reported by Donaghe and Townsend (1975) and USBR (1963) 


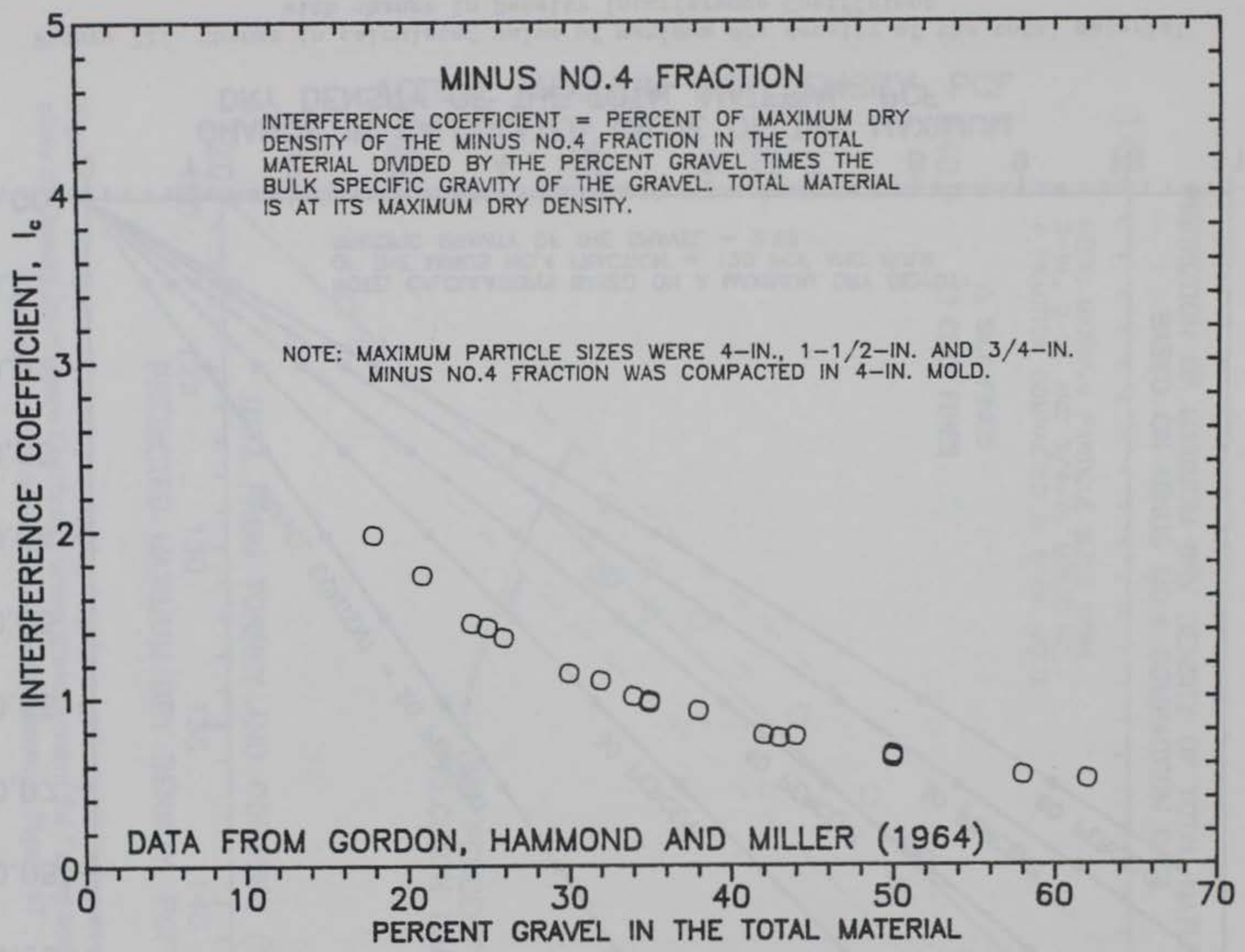

Figure 71. Density Interference Coefficient based on the minus No. 4 fraction versus gravel content of the total material calculated from the compaction data reported by Gordon, Hammond, and Miller (1964) 


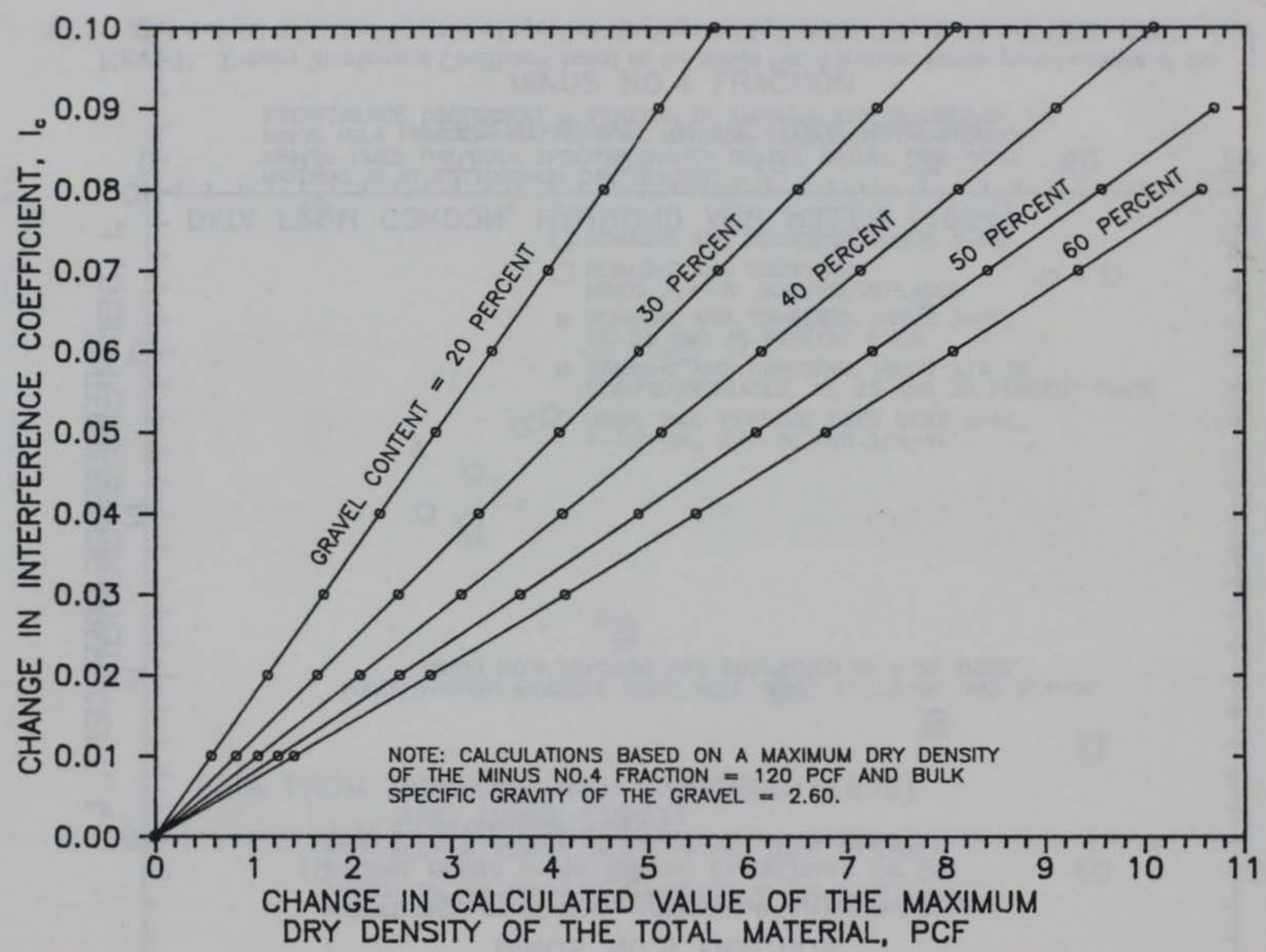

Figure 72. Change in calculated value of maximum dry density of the total material with change in Density Interference Coefficient 


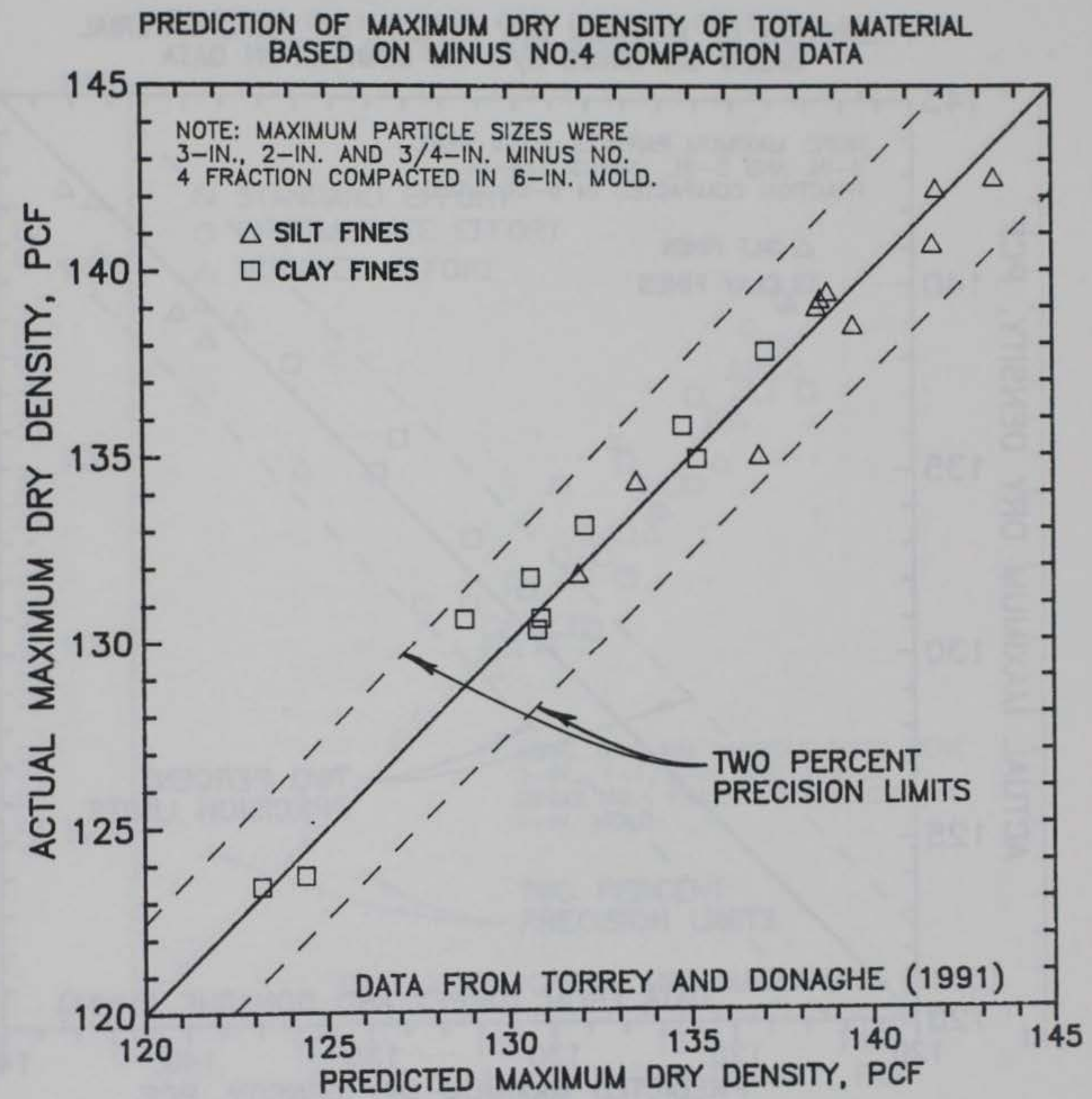

Figure 73. Prediction of maximum dry density of the total material using an estimated-fit curve of Density Interference Coefficient versus gravel content for Torrey and Donaghe's data based on the minus No. 4 fraction shown in Figure 67 
PREDICTION OF MAXIMUM DRY DENSITY OF TOTAL MATERIAL

BASED ON MINUS $3 / 4-I N$. COMPACTION DATA

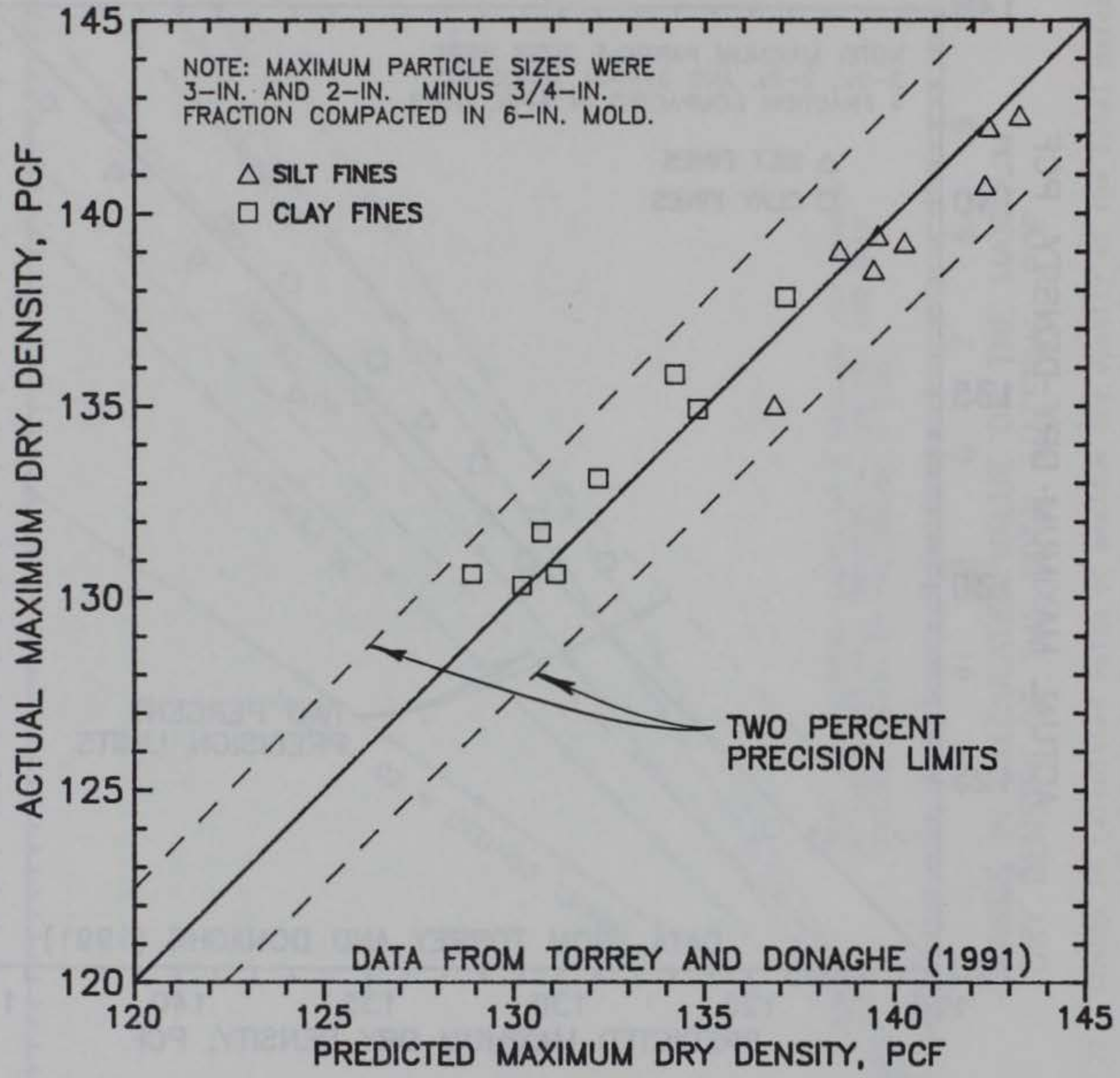

Figure 74. Prediction of maximum dry density of the total material using an estimated-fit curve of Density Interference Coefficient versus gravel content for Torrey and Donaghe's data based on the minus 3/4-in. fraction shown in Figure 68 
PREDICTION OF MAXIMUM DRY DENSITY OF TOTAL MATERIAL

BASED ON MINUS NO.4 COMPACTION DATA

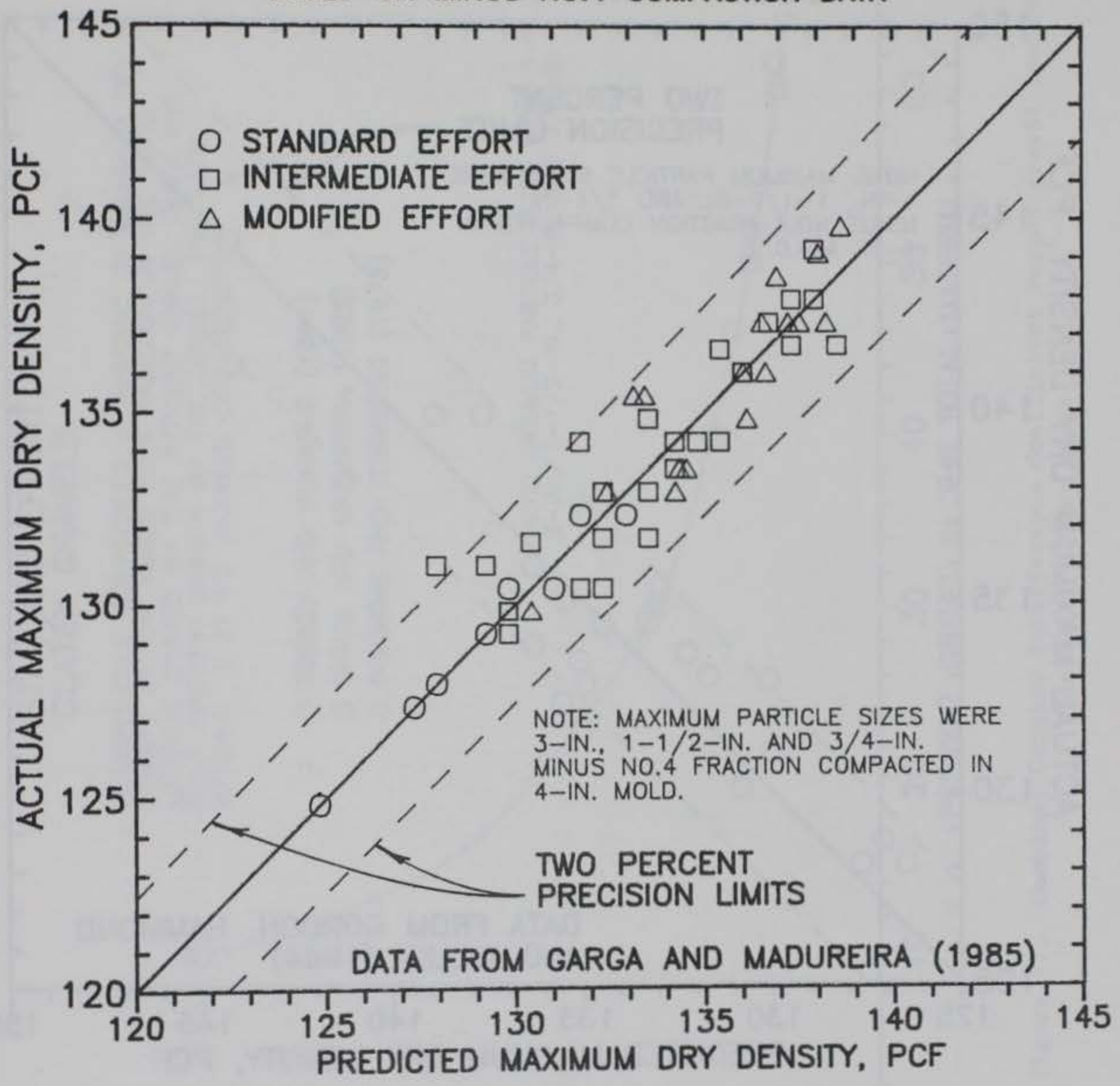

Figure 75. Prediction of maximum dry density of the total material using an estimated-fit curve of Density Interference Coefficient versus gravel content for Garga and Madureira's data based on the minus No. 4 fraction shown in Figure 69 


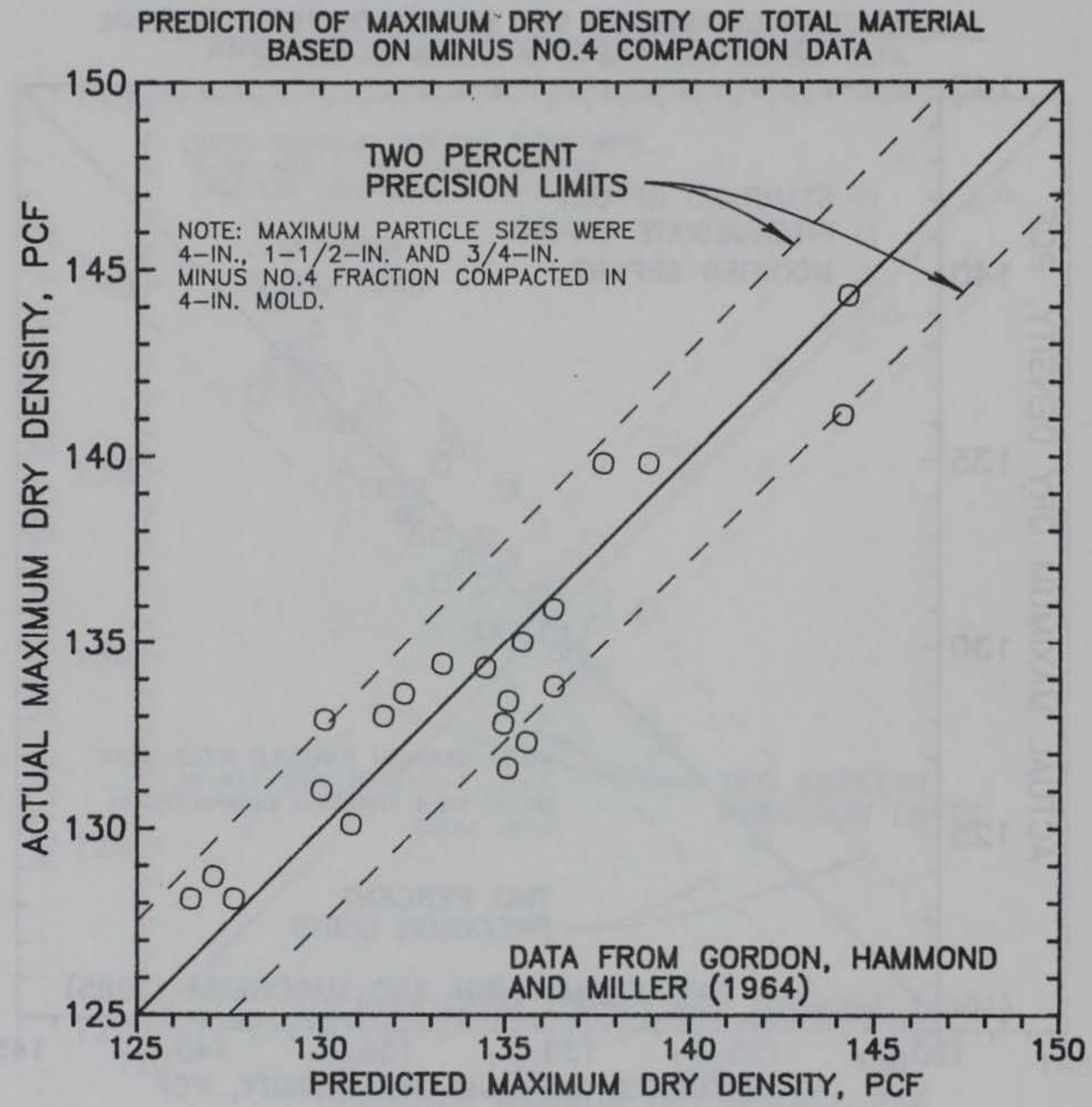

Figure 76. Prediction of maximum dry density of the total material using an estimated-fit curve of Density Interference Coefficient versus gravel content for Gordon, Hammond, and Miller's data based on the minus No. 4 fraction shown in Figure 71 


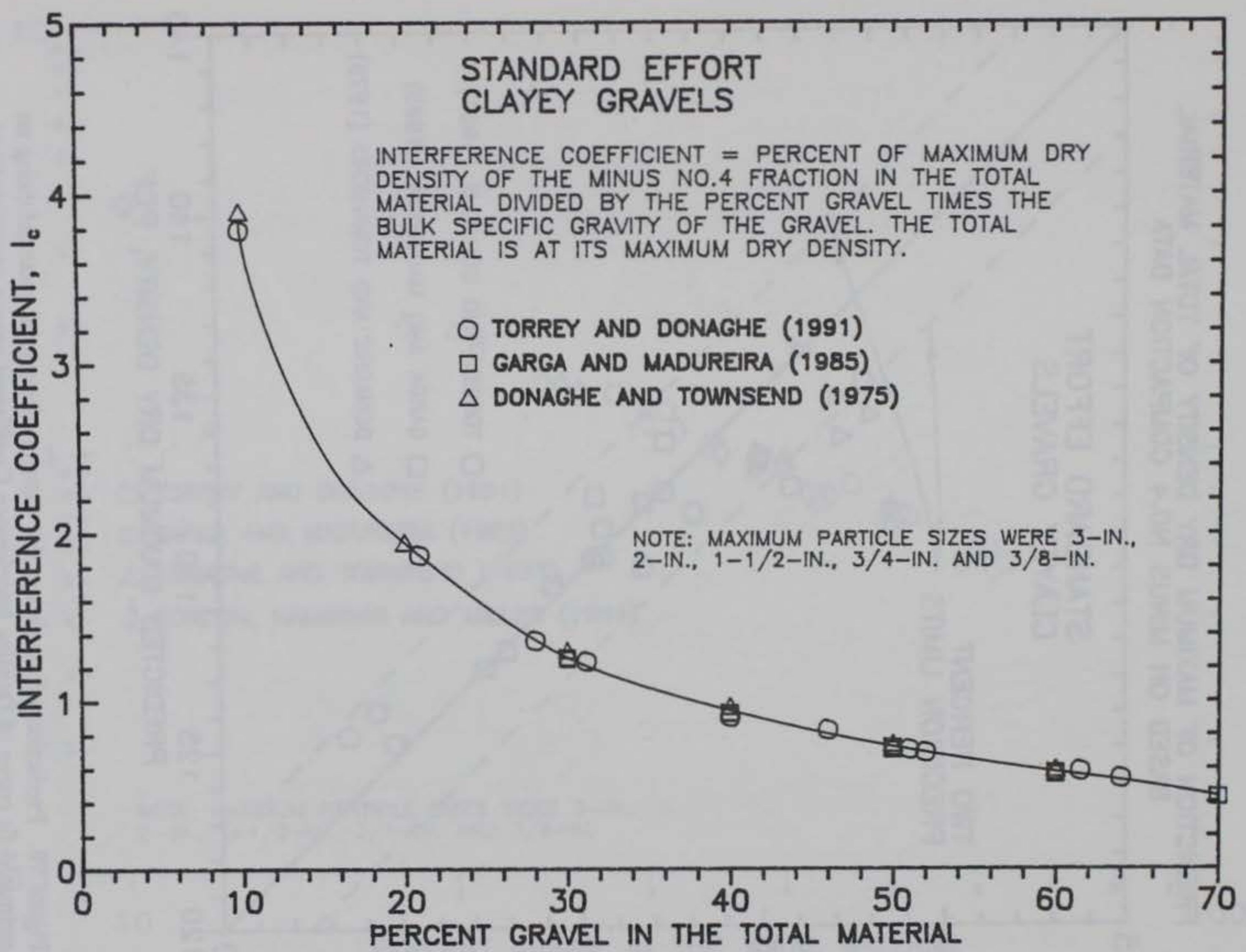

Figure 77. Combined Density Interference Coefficient versus gravel content data for clayey gravels 


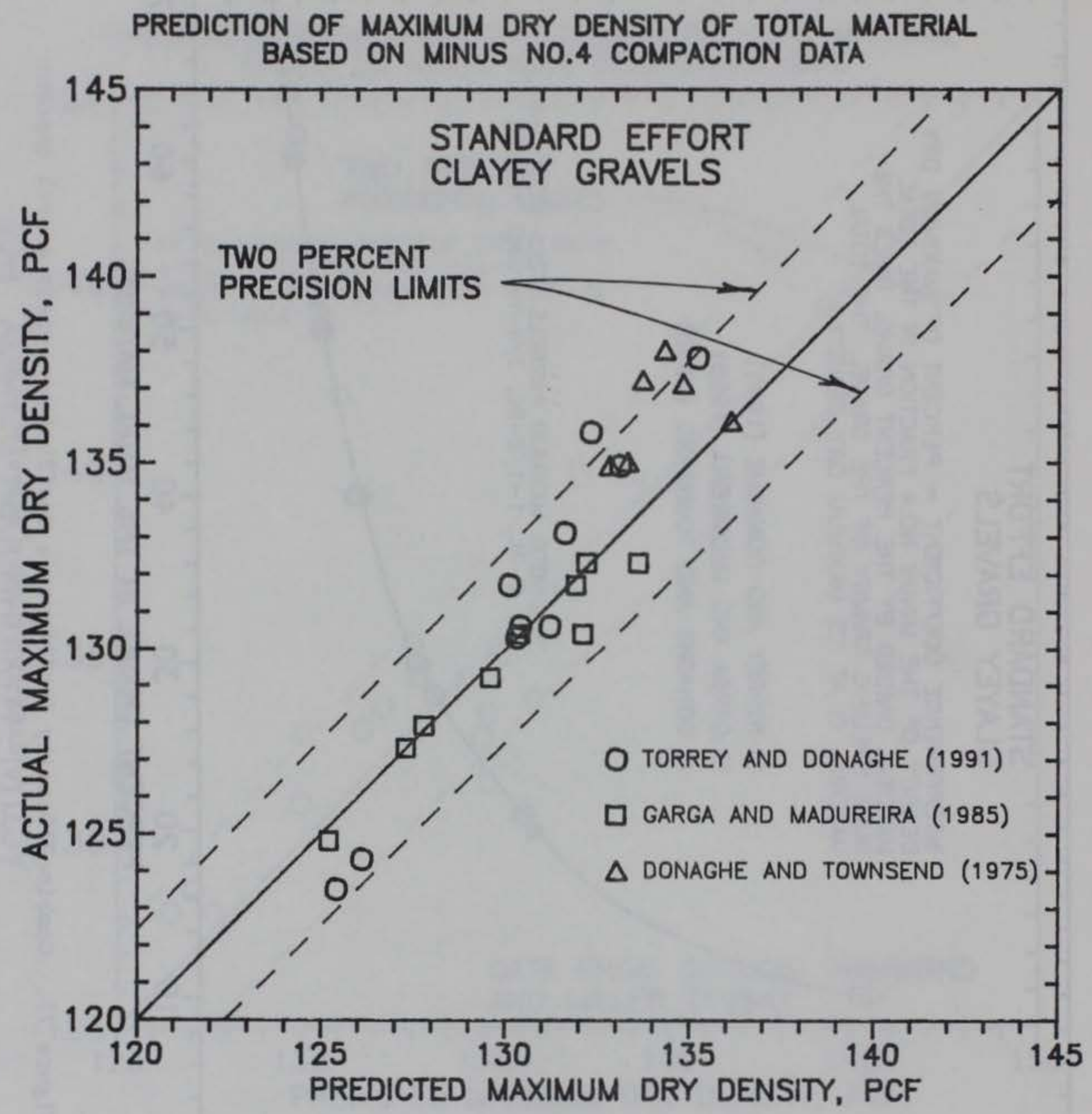

Figure 78. Prediction of maximum dry density of the total material using an estimated-fit curve of Density Interference Coefficient versus gravel content for clayey gravels of Figure 77 


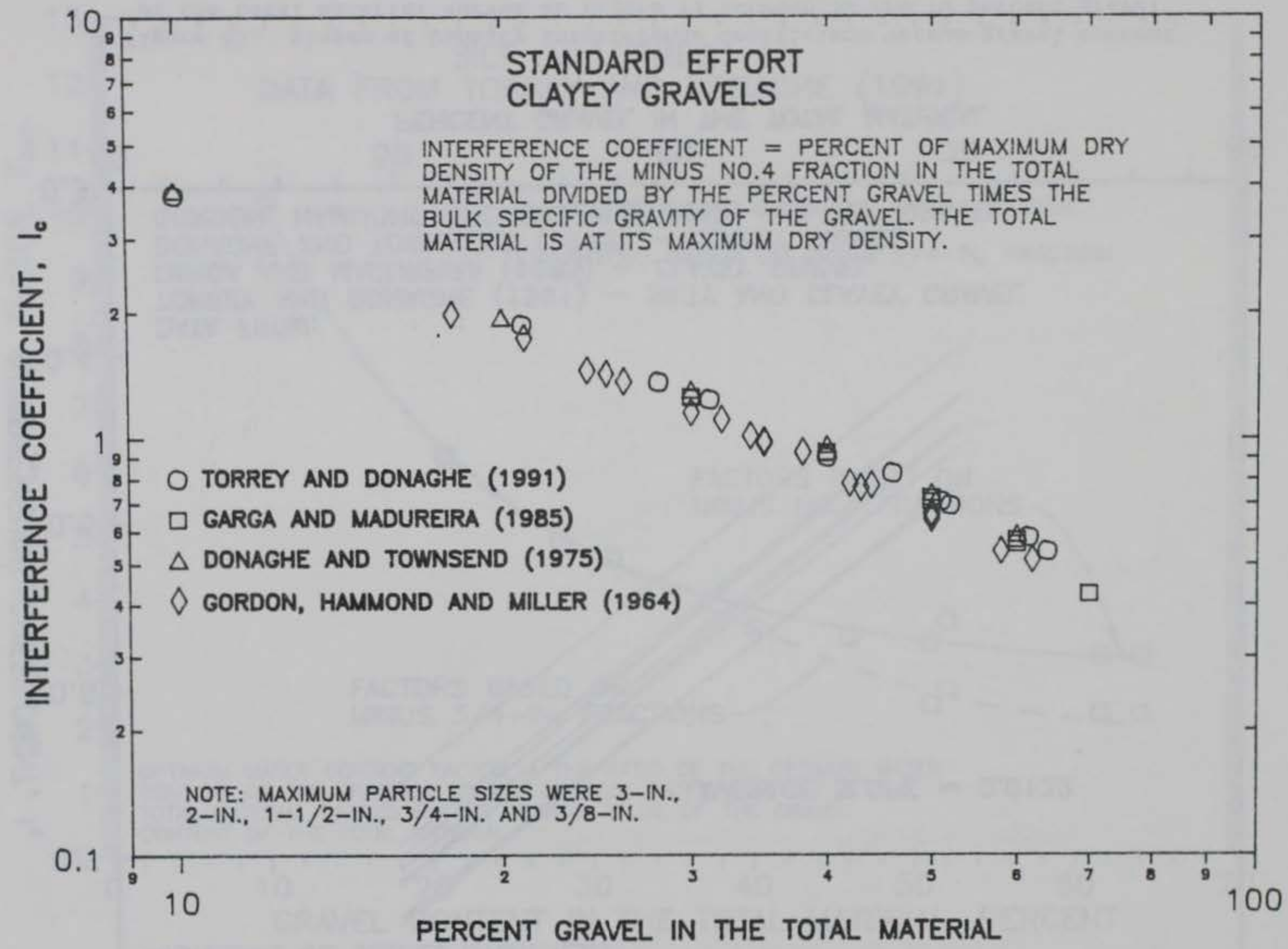

Figure 79. Density Interference Coefficient versus gravel content of the total material plotted in $\log \log$ coordinates 


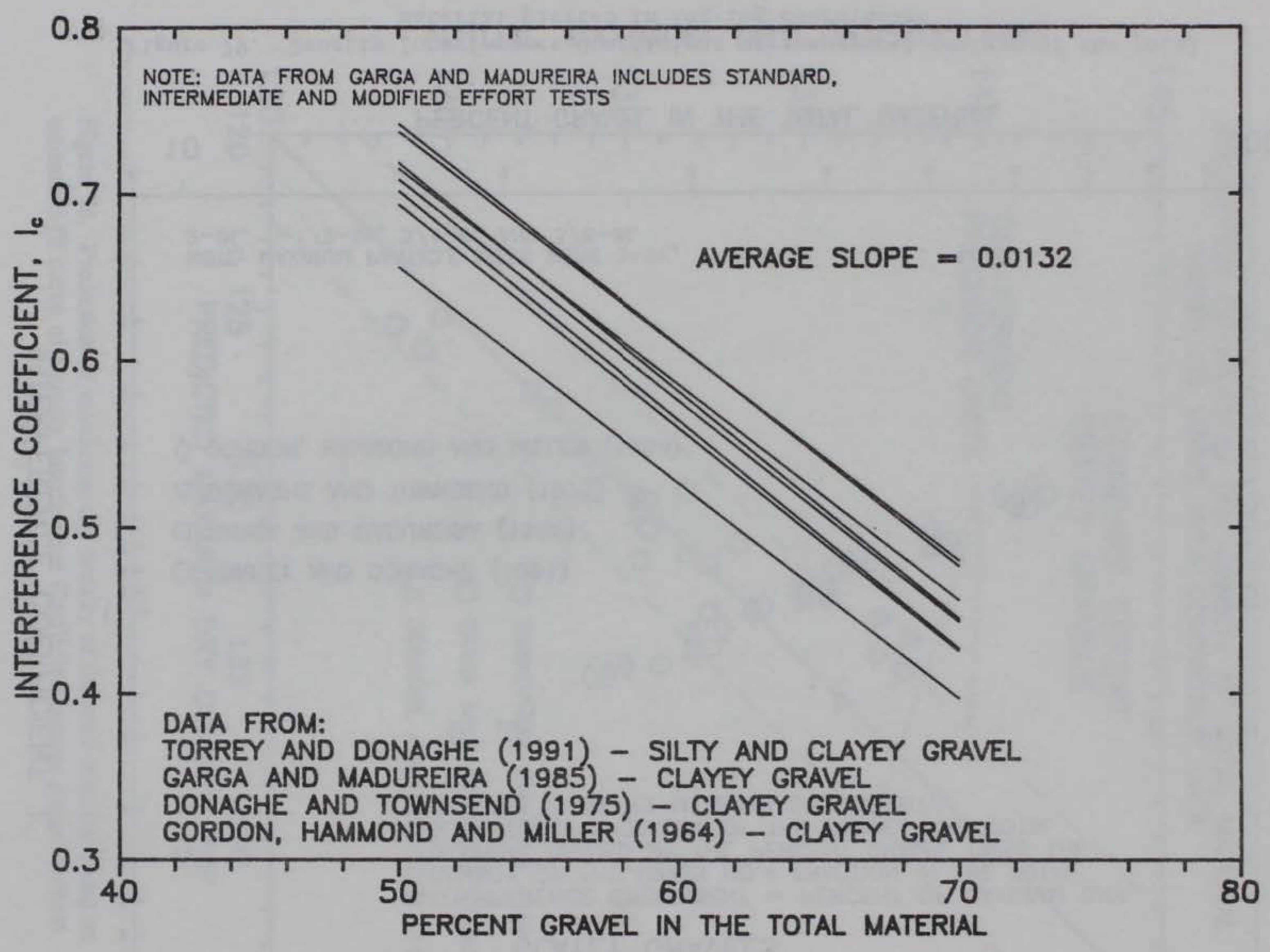

Figure 80. Slopes of Density Interference Coefficient versus gravel content of the total material curves of Figure 79 between 50 and 70 percent gravel 


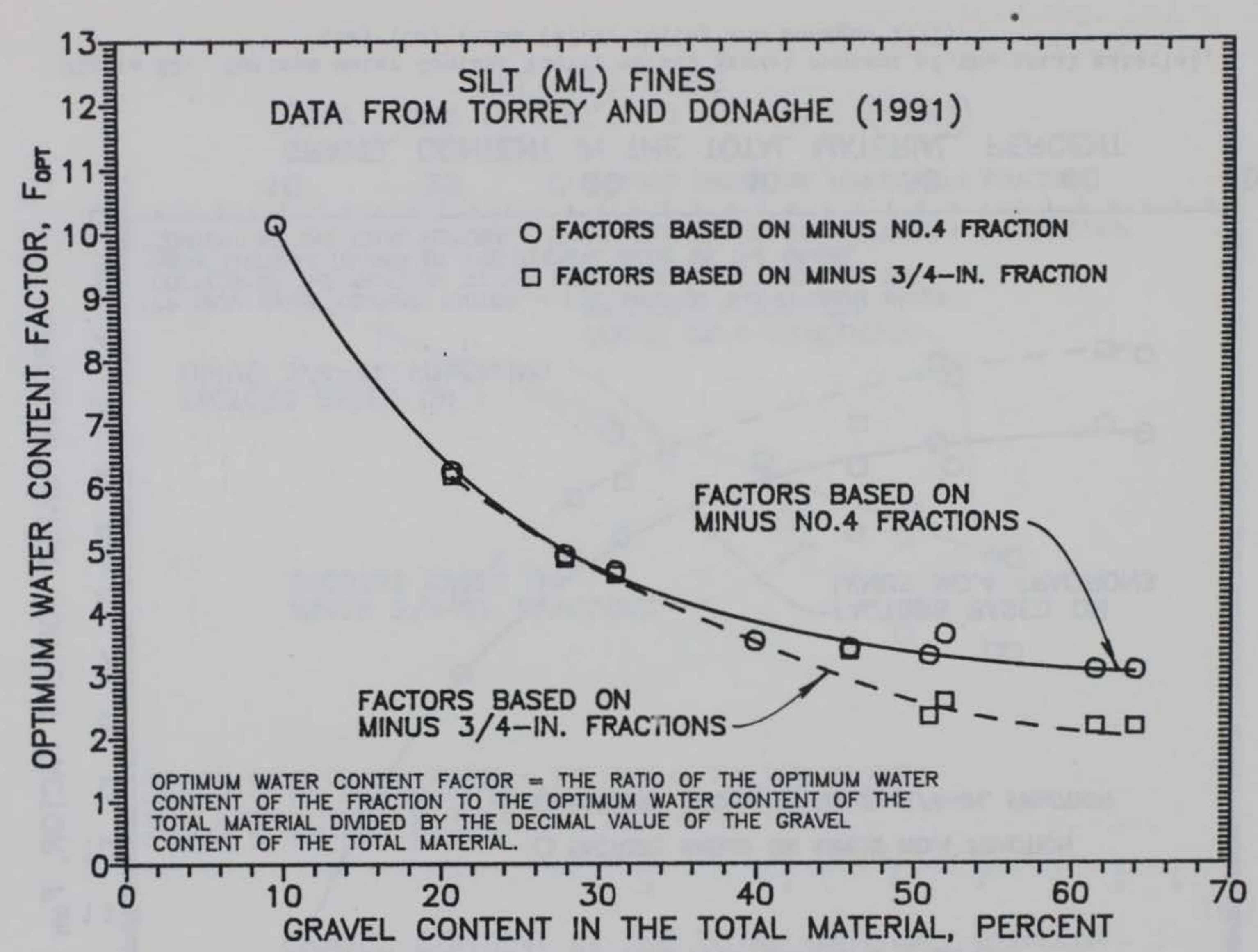

Figure 81. Optimum Water Content Factor versus gravel content of the total material, silt (ML) fines (after Torrey and Donaghe 1991) 


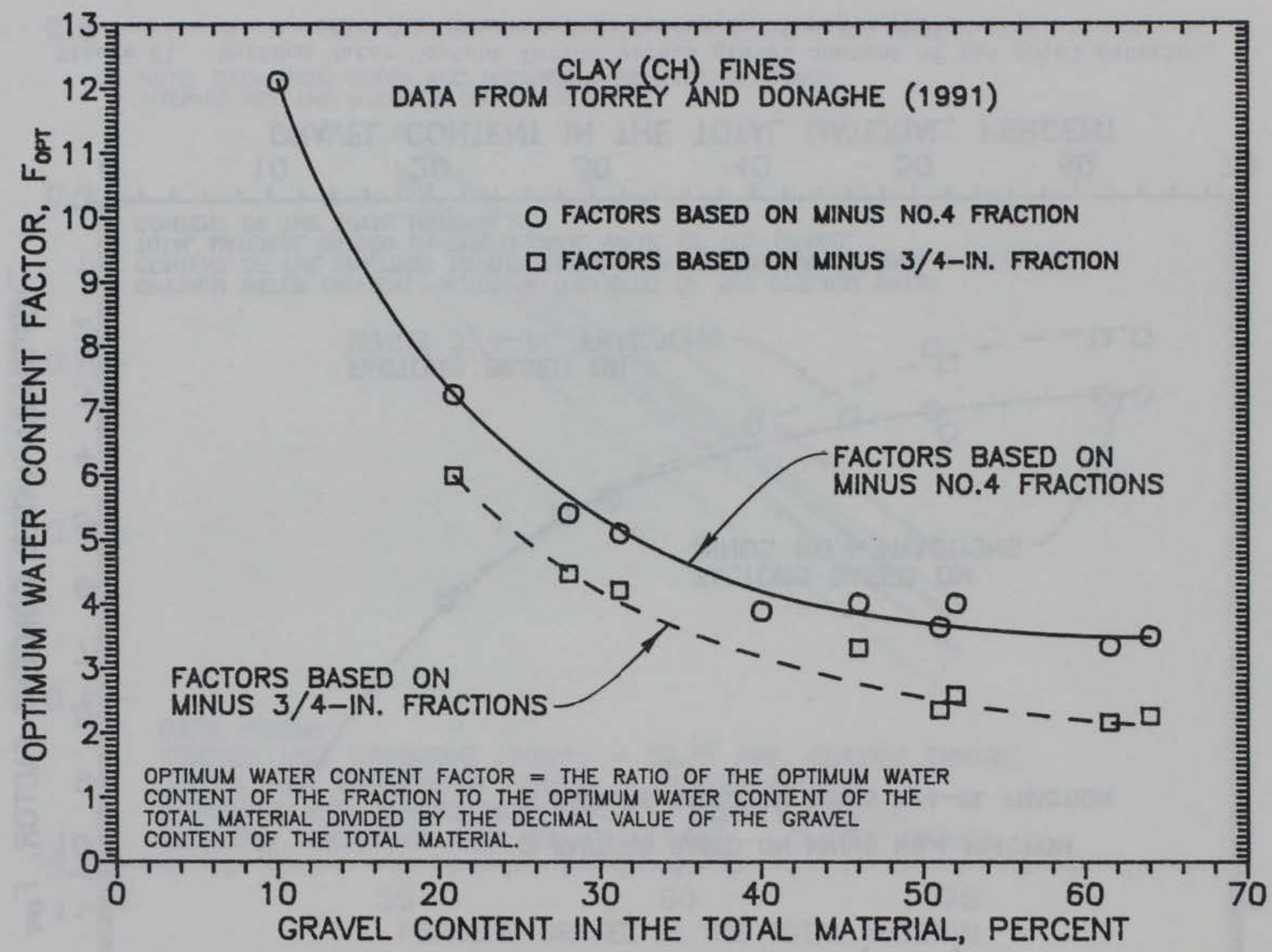

Figure 82. Optimum Water Content Factor versus gravel content of the total material, clay (CH) fines (after Torrey and Donaghe 1991) 


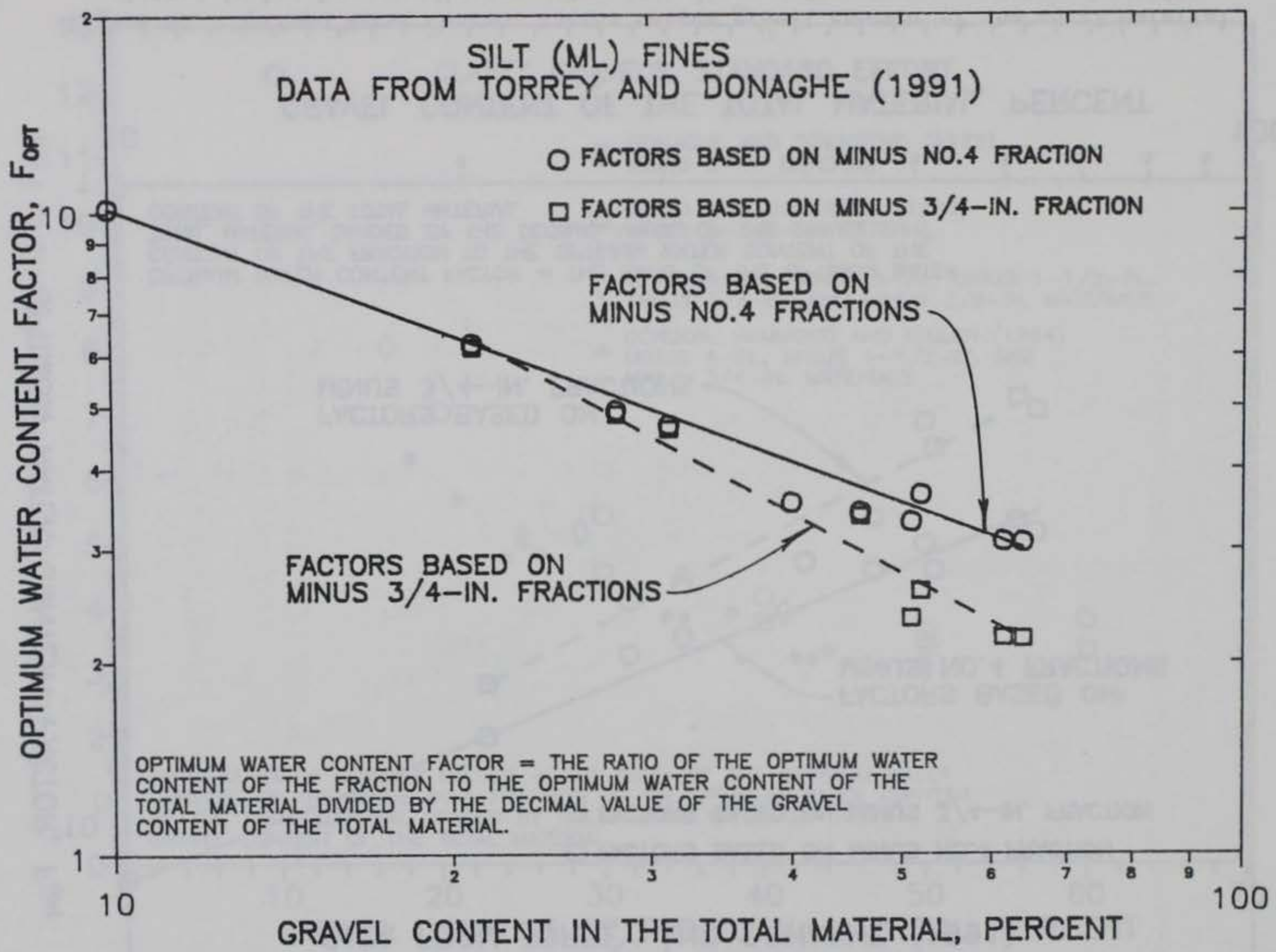

Figure 83. Optimum Water Content Factor versus gravel content of the total material plotted in $\log -\log$ coordinates, silt (ML) fines (after Torrey and Donaghe 1991) 


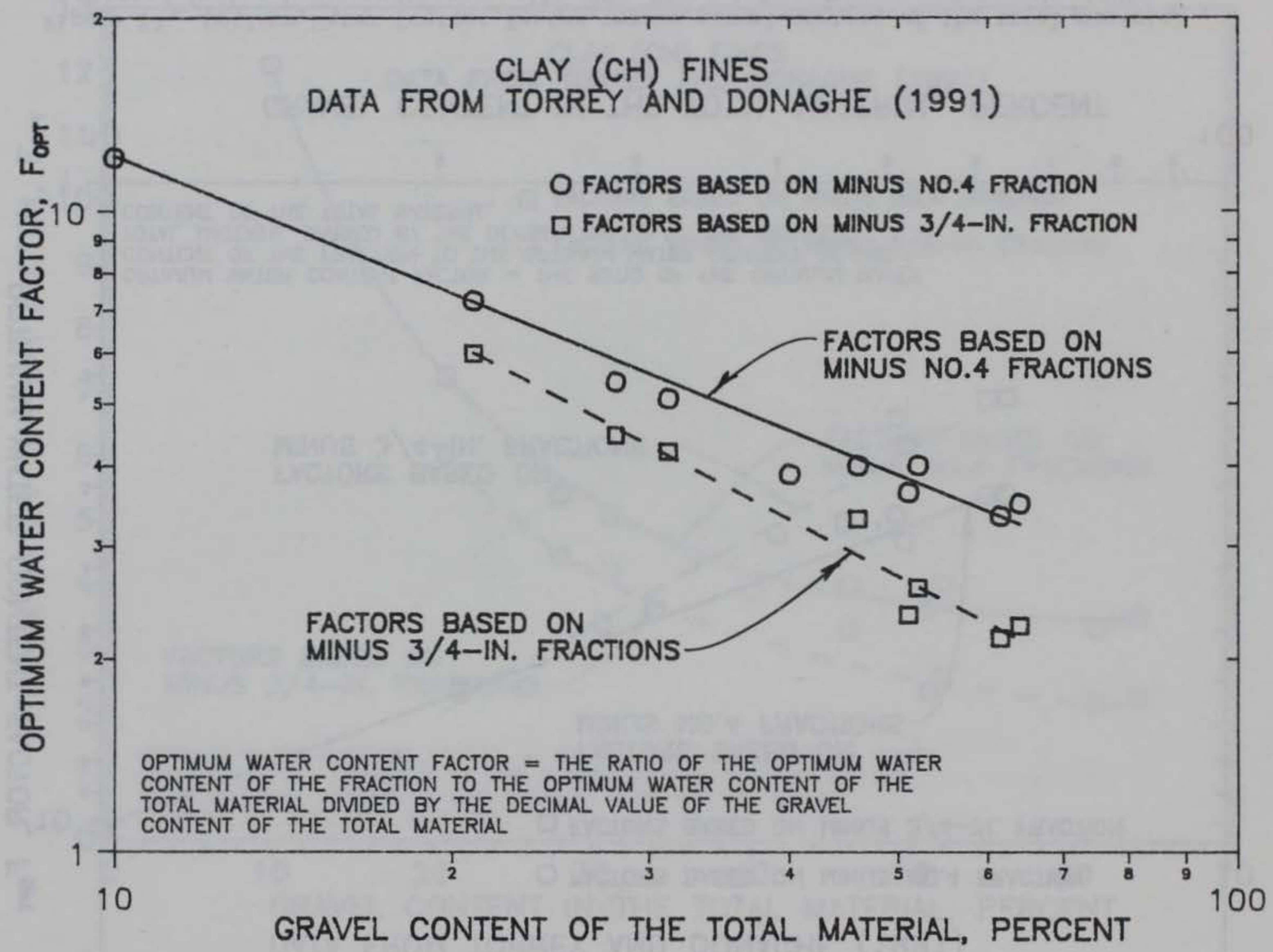

Figure 84. Optimum Water Content Factor versus gravel content of the total material, plotted in log-log coordinates, clay (CH) fines (after Torrey and Donaghe 1991) 


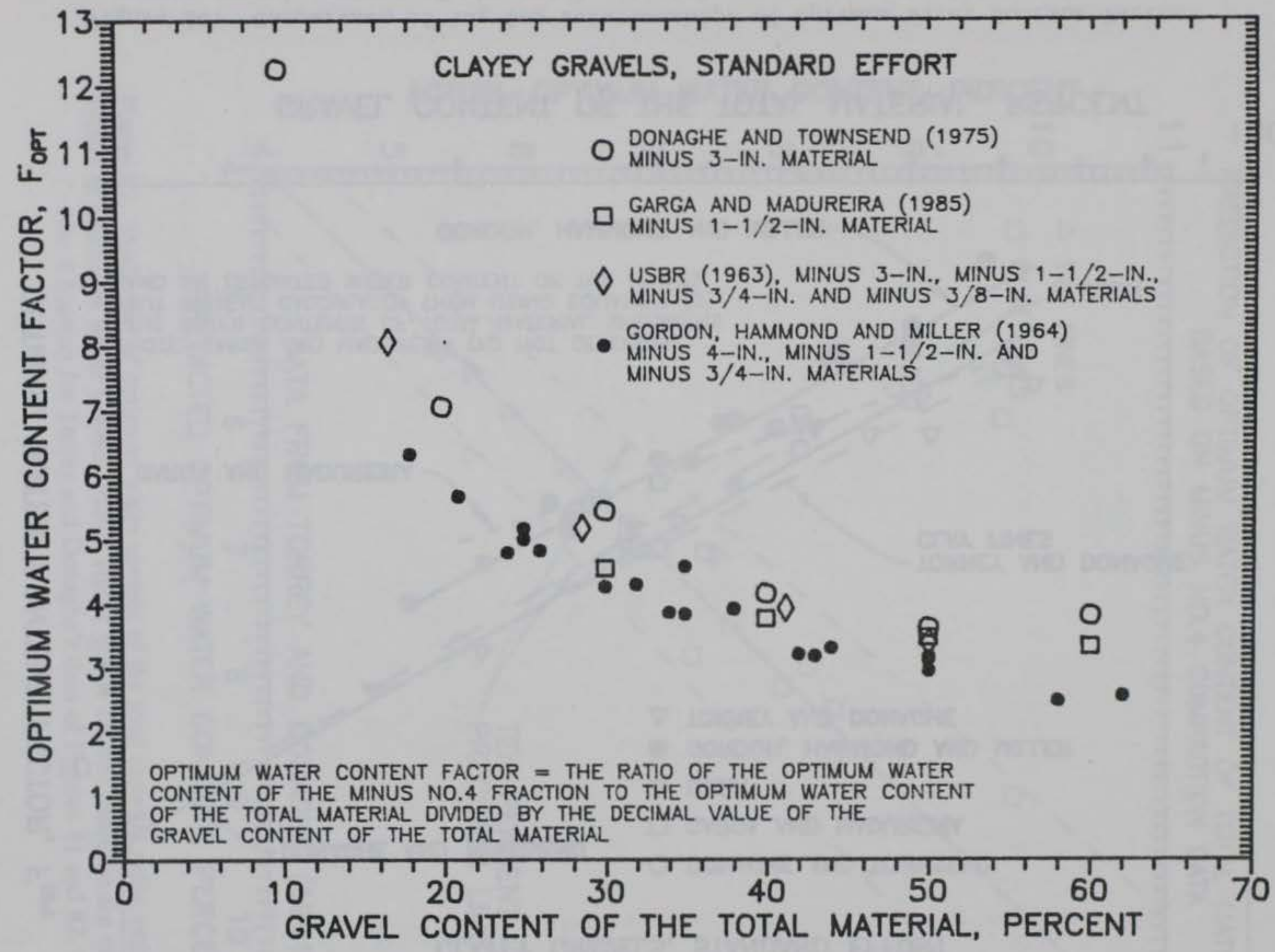

Figure 85. Combined plot of Optimum Water Content Factor versus gravel content of the total material for clayey gravels 


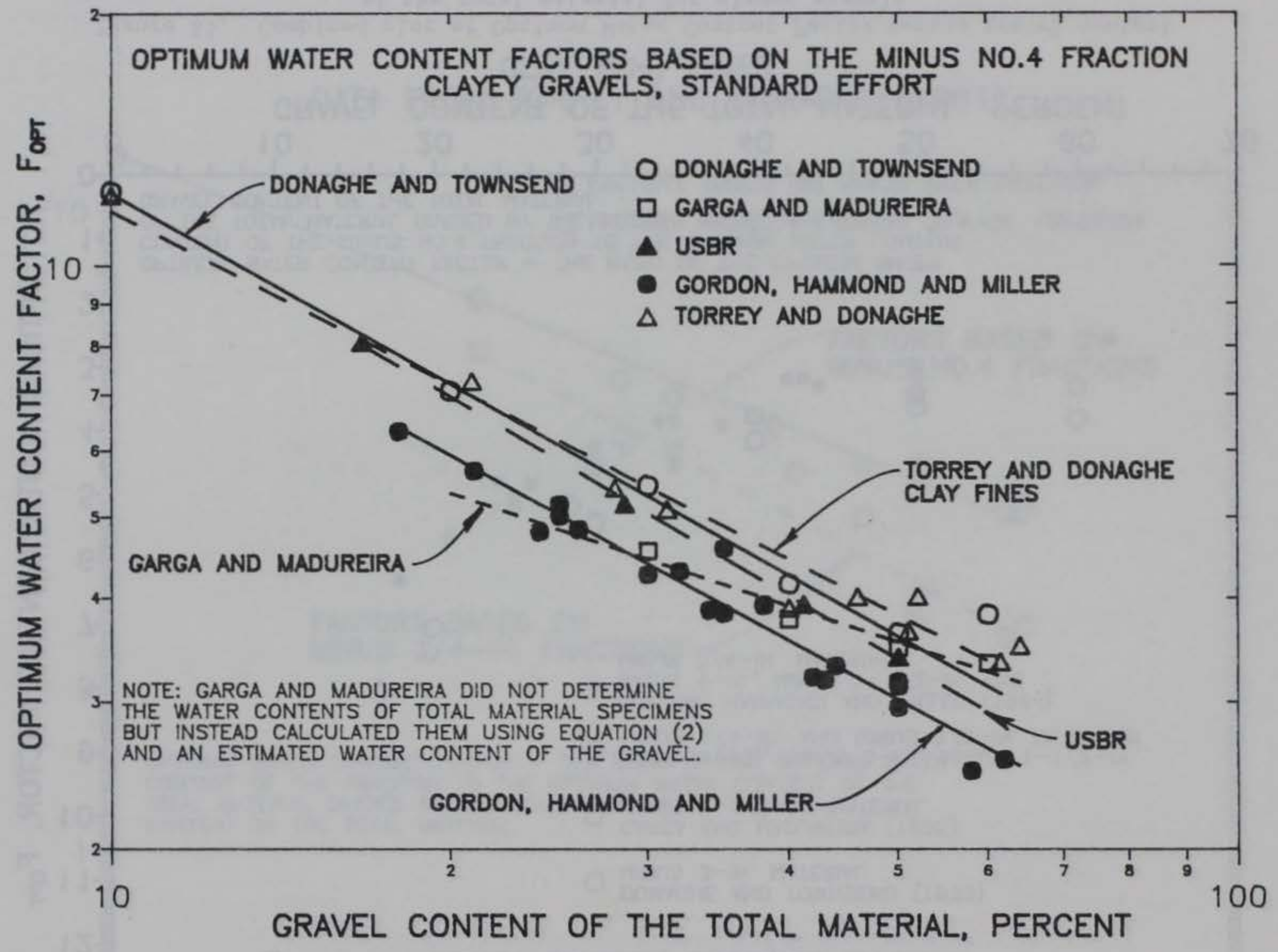

Figure 86. Comparison of $\log -\log$ relationships of Optimum Water Content Factor versus gravel content of the total material among the several investigators 
PREDICTION OF OPTIMUM WATER CONTENT OF TOTAL MATERIAL BASED ON MINUS NO.4 COMPACTION DATA

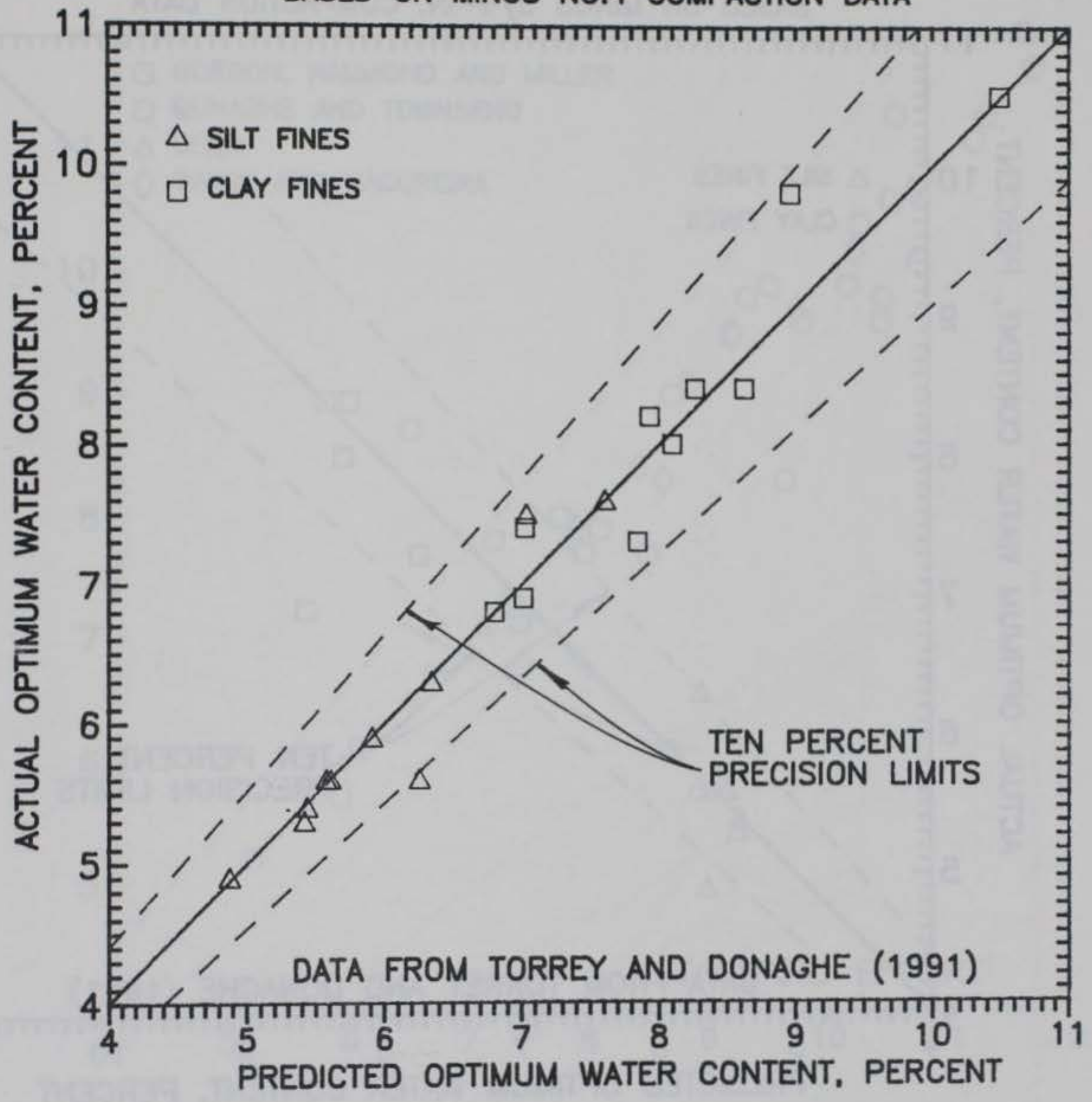

Figure 87. Prediction of optimum water content of the total material using estimated-fit curves of Optimum Water Content Factor versus gravel content based on the minus No. 4 fraction for Torrey and Donaghe's data of Figures 81 and 82 


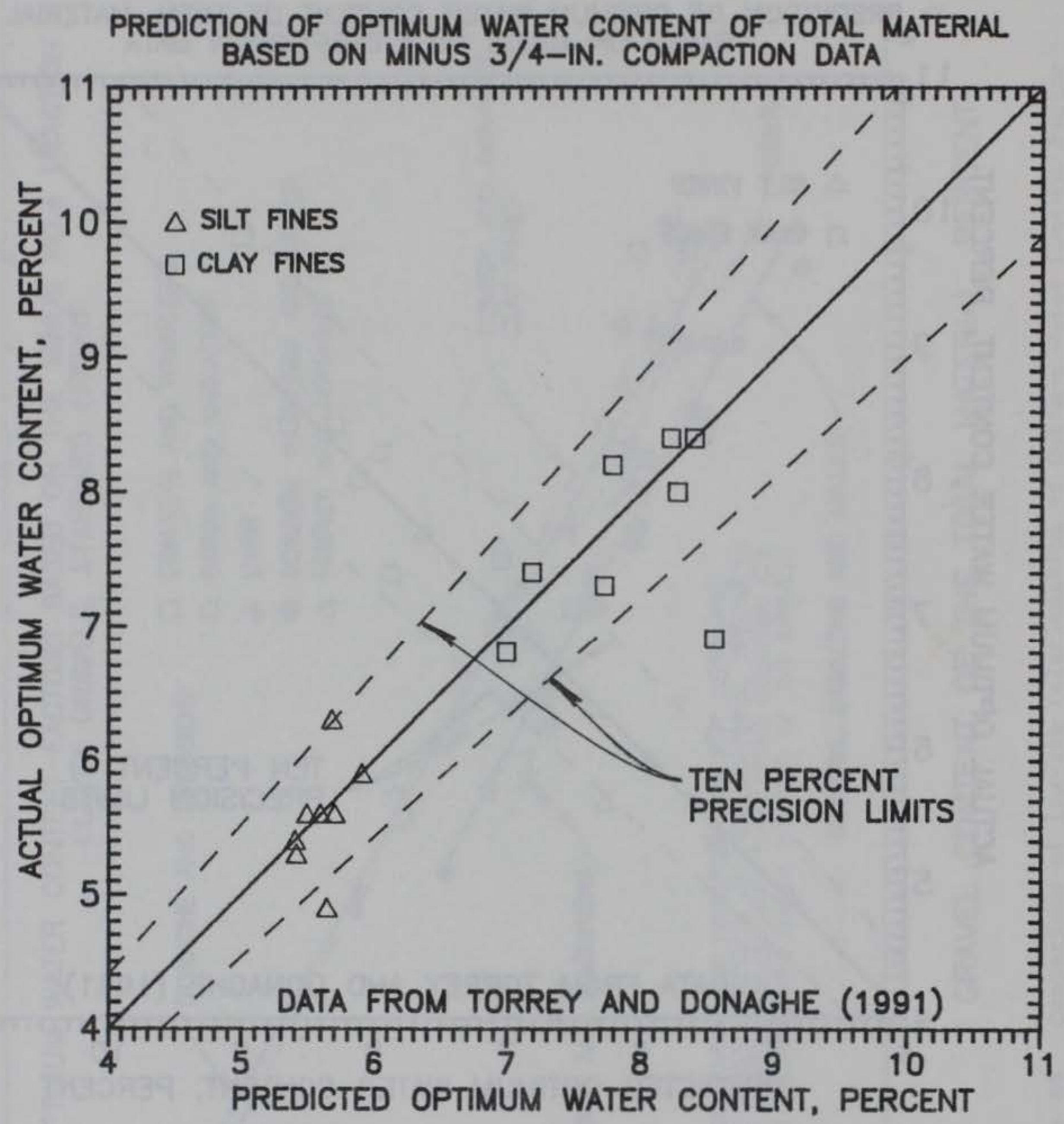

Figure 88. Prediction of optimum water content of the total material using estimated-fit curves of Optimum Water Content Factor versus gravel content based on the minus 3/4-in. fraction for Torrey and Donaghe's data of Figures 81 and 82 


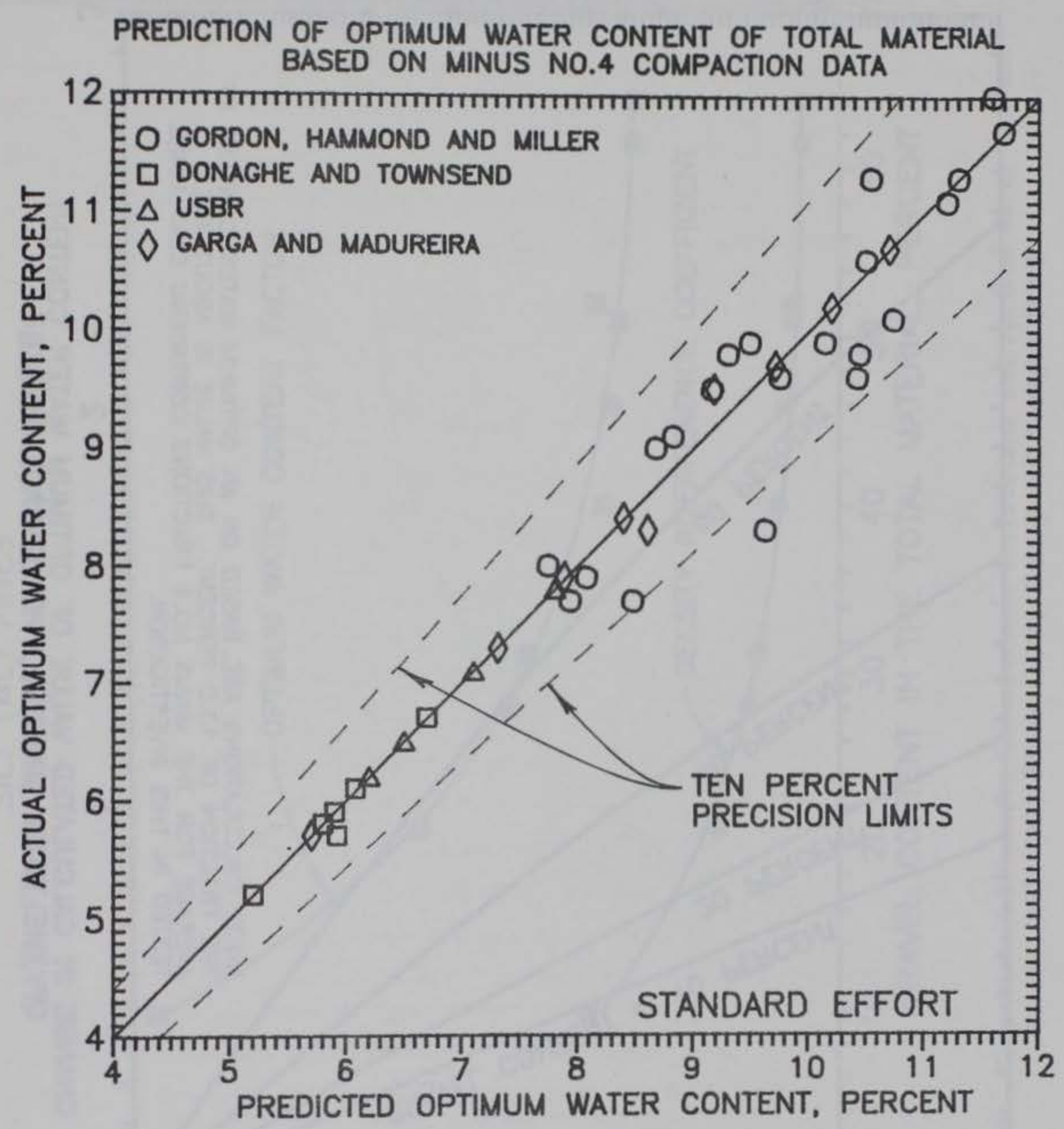

Figure 89. Prediction of optimum water content of the total material using estimated-fit curves of Optimum Water Content Factor versus gravel content for each individual data set of Figure 85 


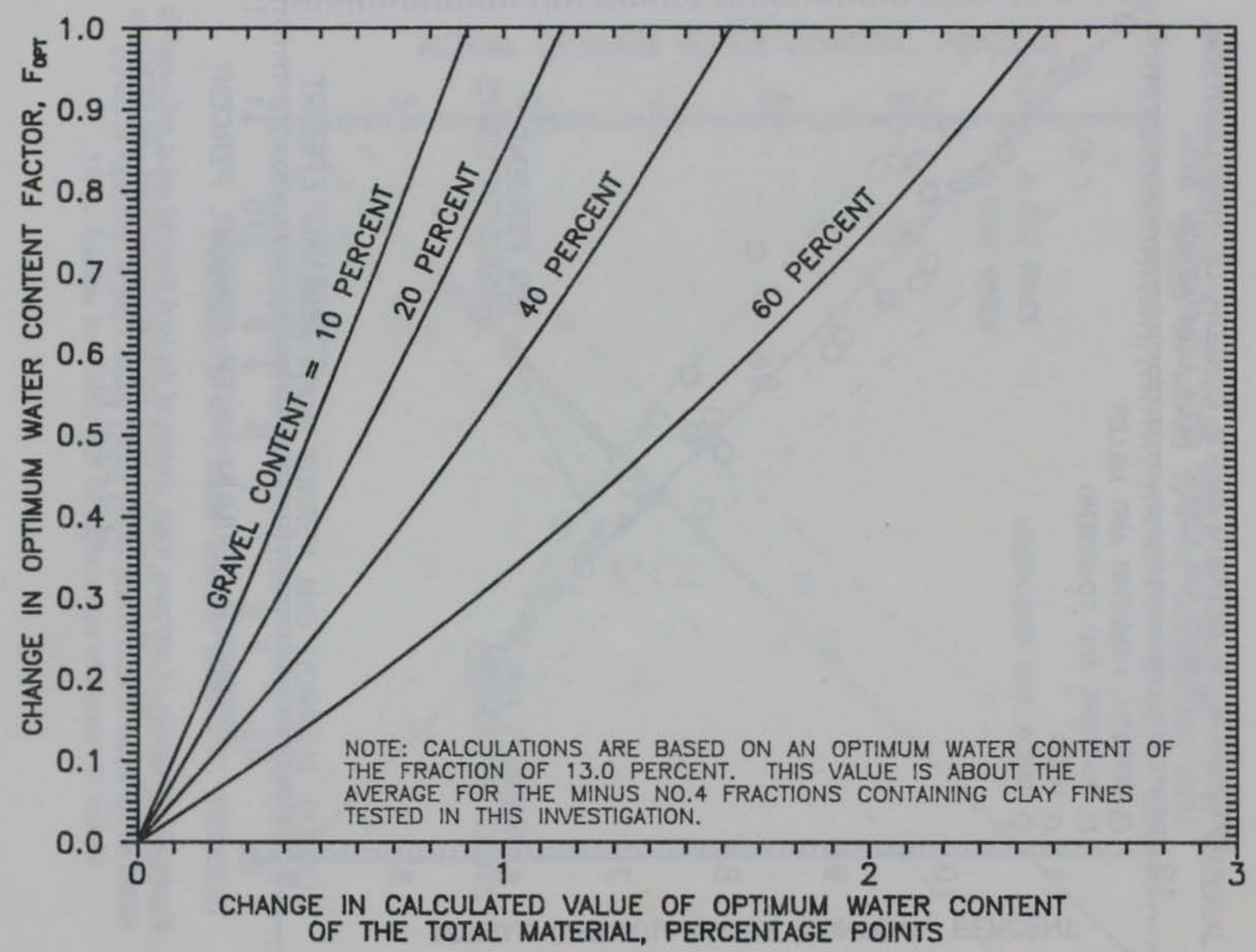

Figure 90. Sensitivity of predicted value of optimum water content to change in value of Optimum Water Content Factor 


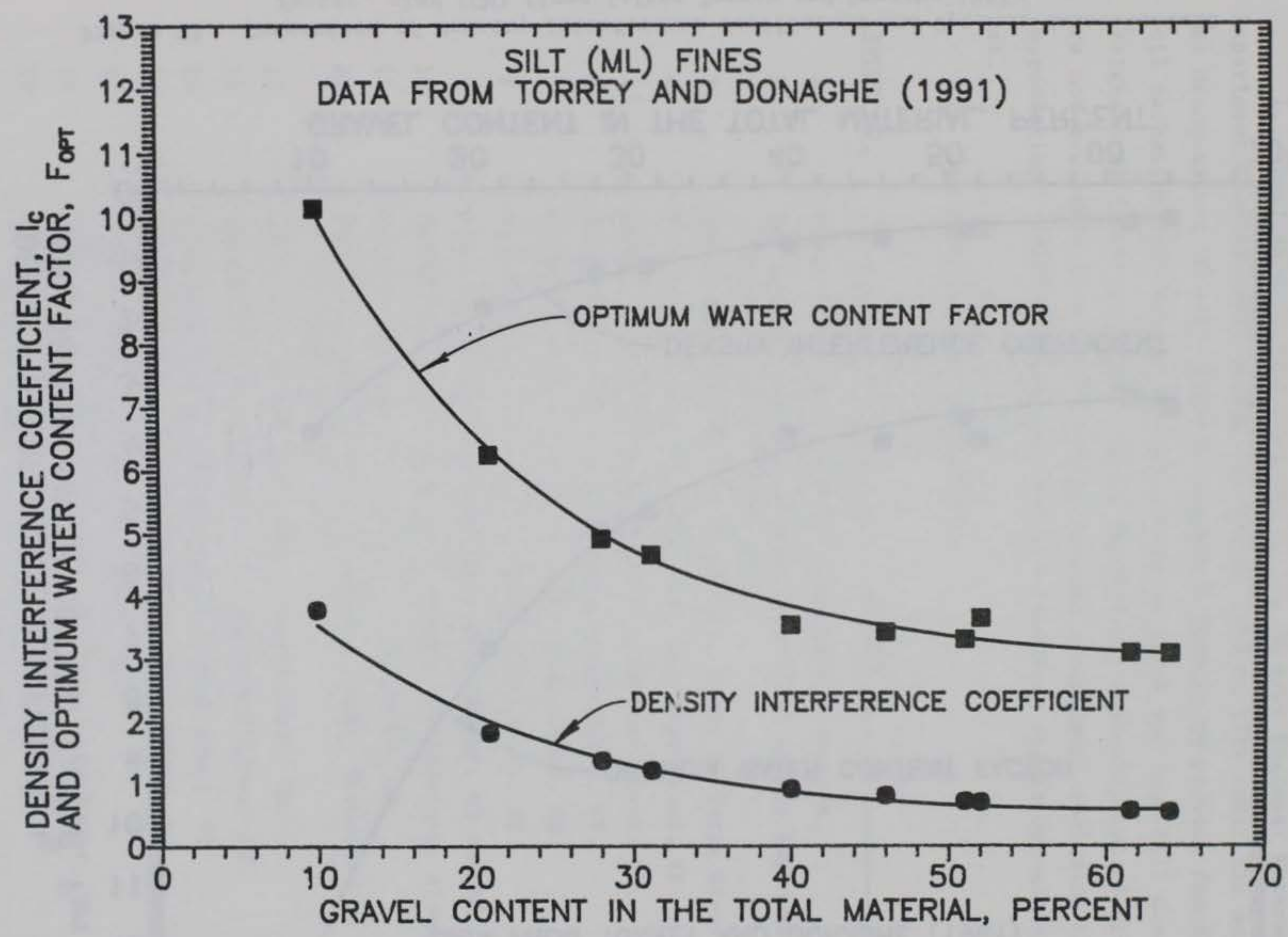

Figure 91. Comparison of Density Interference Coefficient and Optimum Water Content Factor, silt (ML) fines (after Torrey and Donaghe 1991) 


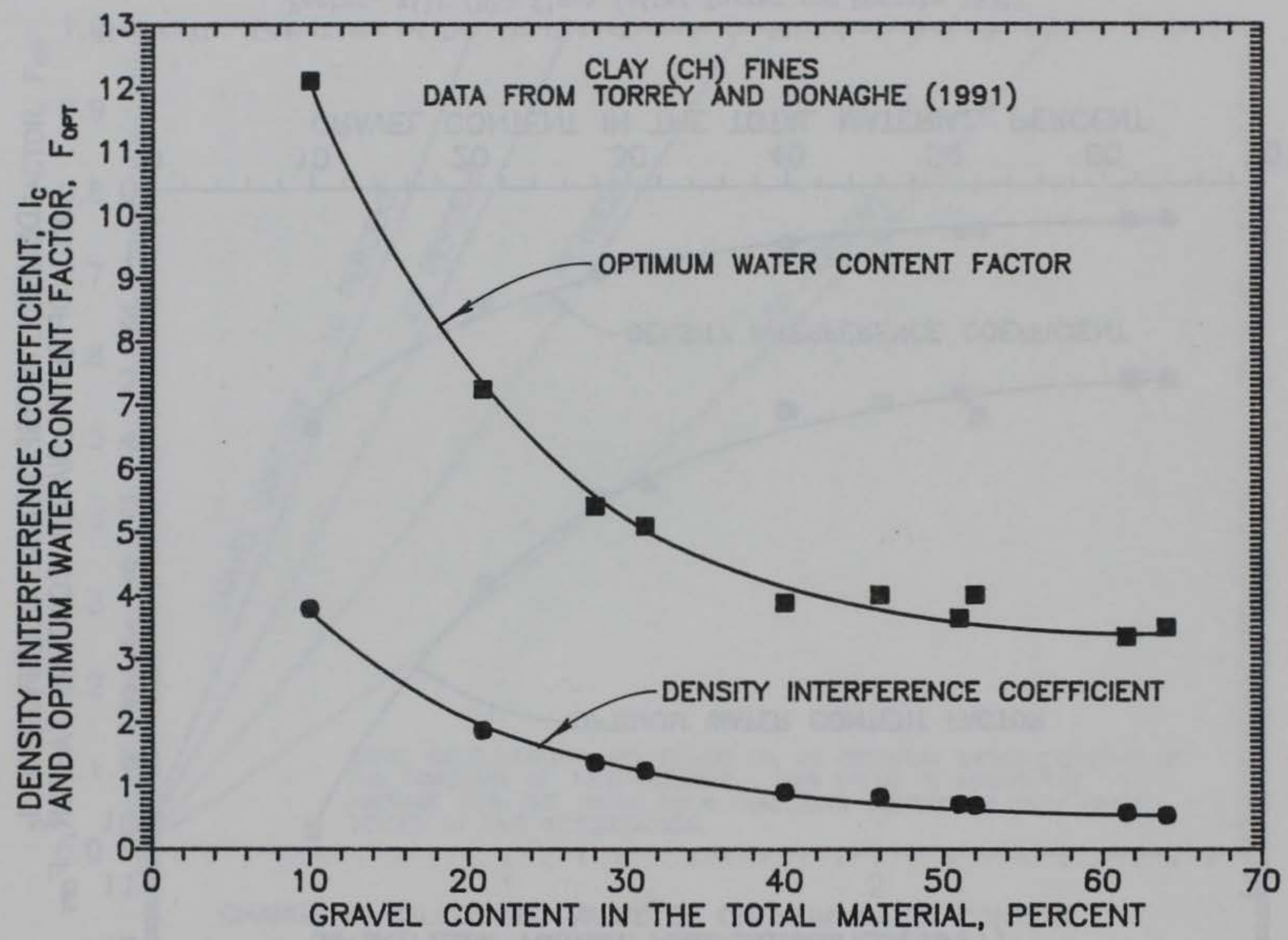

Figure 92. Comparison of Density Interference Coefficient and Optimum Water Content Factor, clay (CH) fines (after Torrey and Donaghe 1991) 
1. These reports were prepared by the US Army Engineer Waterways Experiment Station (WES) during the period 1960 to 1974 under the auspices of the Headquarters US Army Corps of Engineers (USACE) to provide foundation and soil mechanics information on representative earth and rock-fill dams to designers of future projects. These reports are currently out of print and are not available from WES except by special arrangement including cost of reproduction. USACE District or Division libraries should contain a complete set.

2. The reports were as follows:

\section{REPORT NO.}

1

Barre Falls Dam and Reservoir, Ware River, MA. San Antonio Dam, San Antonio and Chino Creeks Improvement, Santa Ana River Basin, CA. Pomme de Terre Dam and Reservoir, Pomme de Terre River, MO. Tuttle Creek Dam and Reservoir, Big Blue River, KS. Coyote Valley Dam and Reservoir, Russian River, CA. Rough River Dam and Reservoir, Rough River, KY. Otter Brook Dam and Reservoir, Otter Brook, NH. Painted Rock Dam and Reservoir, Gila River, AZ. Table Rock Dam and Reservoir, White River, MO and AR. Buckhorn Dam and Reservoir, Middle Fork Kentucky River, KY. Coralville Dam and Reservoir, Iowa River, IA. Jadwin Dam and Reservoir, Dyberry Creek, Tributary of Lackawaxen River, PA.

Terminus Dam and Reservoir, Kaweah River, CA. Thomaston Dam and Reservoir, Naugatuck River, CT. Ball Mountain Dam and Reservoir, West River, VT. Alvin R. Bush Dam and Reservoir, West River, VT. Everett Dam, Piscataquog River, NH. John W. Flannagan Dam and Reservoir, Pound River, VA. Abiquiu Dam and Reservoir, Rio Chama, NM. Howard A. Hanson Dam, Green River, WA. 
REPORT NO.

Francis E. Walter Dam, Lehigh River, PA.

Lucky Peak Dam, Boise River, IA.

Lookout Point Dam, Middle Fork, Willamette River, OR.

North Hartland Dam, Ottauquechee River, VT.

W. Kerr Scott Dam, Yadkin River, NC.

New Hogan Dam and Reservoir, Calaveras River, CA.

Belton Dam and Reservoir, Leon River, TX.

Buford Dam, Chattachoochee River, GA.

Cougar Dam, South Fork, McKenzie River, OR.

Mad River Dam, Mad River, CT.

Success Dam, Tule River, CA.

Black Butte Dam, Stony Creek, CA.

Littleville Dam, Middle Branch, Westfield River, MA.

Nolin River Dam, Nolin River, KY.

Canyon Dam and Reservoir, Guadalupe River, TX.

North Fork of Pound Dam and Reservoir, North Fork of Pound River, VA.

Curwensville Dam and Reservoir, West Branch Susquehanna River, Curwensville, PA.

Hills Creek Dam, Middle Fork, Willamette River, OR.

Wilson Dam and Reservoir, Saline River, KS.

Summersville Dam, Gauley River, W.VA.

East Branch Dam, Clairon River, PA.

Oahe Dam and Reservoir, Missouri River, SD.

Kinzua Dam, Allegheny River, PA.

Somerville Dam and Reservoir, Yegua Creek, TX.

Mississinewa Dam, Mississinewa River, IN.

J. Percy Priest Dam and Reservoir, Stones River, TN.

Stillhouse Hollow Dam and Reservoir, Lampasas River, TX.

Alamo Dam and Reservoir, Bill Williams River, AZ.

Blue River Dam, Blue River, OR.

Eau Galle Dam, Eau Galle River, WI.

Green River Dam, Green River, KY.

Galisteo Dam and Lake, Galisteo Creek, NM. 
REPORT NO.

53

54

55

56

57

58

59

60

61

62

63

64

65

66

67

DeGray Dam and Lake, Caddo River, AR.

Garrison Dam, Missouri River, ND.

Stockton Dam, Sac River, MO.

Cottonwood Springs Dam, Fall River Basin, SD.

Fall Creek Dam, Fall Creek, OR.

Foster Dam and Lake, South Santiam River, OR.

Black Rock Dam and Lake, Branch Brook, CT.

Colebrook River Dam and Lake, West Branch Farmington River, CT.

Sam Rayburn Dam and Lake, Angelina River, TX.

Broken Bow Dam and Lake, Mountain Fork River, OK.

Pine Creek Dam and Lake, Little River, OK.

Aylesworth Creek Dam and Lake, Lackawanna River, PA.

Foster Joseph Sayers Dam and Lake, West Branch, Susquehanna River, PA.

Fishtrap Dam and Lake, Levisa Fork, Big Sandy River, KY.

Lake Red Rock, Des Moines River, IA. 


\section{APPENDIX B: DETERMINING THE WATER CONTENT OF} THE OVERSIZED FRACTION

1. This Appendix describes a procedure for determining the water content of the oversized fraction of an earth-rock mixture for use in the following equation which is typically used for calculating the water content of a total material from that of a fraction or vice versa:

$$
W_{t}=f W_{f}+C W_{c}
$$

or

$$
W_{f}=\frac{W_{t}-C W_{c}}{f}
$$

where

$$
\begin{aligned}
W_{t} & =\text { water content of the total material, percent } \\
W_{f} & =\text { water content of finer fraction, percent } \\
W_{c} & =\text { water content of coarser (oversized) fraction, percent } \\
f & =\text { percent by weight finer fraction } \\
c & =\text { percent by weight coarser (oversized) fraction }
\end{aligned}
$$

2. In estimating the water content of the total material from that of a fraction, it has commonly been the practice to assume the water content of the oversized fraction, $W_{c}$, to be the absorption, $A$, of the gravel. Although not defined in USACE (1970), the absorption A of a gravel is its water content in the saturated surface-dry condition. The saturated surface-dry condition is defined in USACE (1970), Appendix IV, as that state where the smooth portions of a gravel particle are essentially dry while any tiny open voids or "pores" are filled with water. Although a rare case, the saturated surfacedry state would also include water filling any voids in the interior of a particle which may access water from the outside. The absorption, A, may be calculated from the values of apparent and bulk specific gravities as follows: 


$$
A=\frac{G_{\mathrm{a}}-G_{\mathrm{m}}}{G_{\mathrm{a}} G_{\mathrm{m}}} \times 100 \text { percent }
$$

where

$\mathrm{G}_{\mathrm{a}}=$ the apparent specific gravity of the gravel

$\mathrm{G}_{\mathrm{m}}$ - the bulk specific gravity of the gravel

The absorption of a typical gravel which does not exhibit an abundance of tiny open voids in the surfaces of the particles or interior voids which can be filled with water is usually less than 5 percent.

3. There is no reason to believe that the gravel contained within a moist earth-rock mixture retains an amount of water equal to the absorption. At water contents well to the wet side of optimum water content but less than 100 percent saturation, the gravel could conceivably be wetter than the absorption. However, at partially saturated water contents near optimum as is typical of fill placement water contents, it is likely that the water content of the gravel is somewhat less than the absorption. The presumption in using the absorption, A, in Equation (1) or (1a) above is that the difference between the actual water content of the gravel and its absorption is too small to make a significant difference in the calculations especially since the water content of the gravel, $W_{c}$, is multiplied by the percent coarse (oversized) fraction which itself is usually less than 50 percent.

4. The presumption that use of the absorption does not introduce significant error may or may not be true depending on the error as compared to the specified range in placement water content. For instance, if the total range in specified placement water content is three percentage points straddling optimum water content and the error introduced by use of the absorption is one percentage point, that is a very significant error. Even if the error introduced by use of the absorption is only 0.5 percentage points, it isn't so insignificant.

5. It is not prohibitive in time or expense to perform some simple testing to establish a general value for the water content of the oversized fraction as it actually exists in the total materials when those total materials are within the specified range in placement water content. The procedure is outlined as follows: 
a. Obtain representative samples of the materials which include at least the gradations containing the most and least gravel and the largest and smallest maximum particle sizes. At least $500 \mathrm{lb}$ of each sample should be obtained.

b. Spread each sample in flat pans and air-dry the entire sample. Other means, such as ovens and heat lamps, may be used to accelerate drying if the maximum drying temperature is kept below $60 \mathrm{C}$.

c. Reduce all aggregates, or lumps formed during drying, of finegrained material to particles finer than the No. 4 sieve. With a wire brush or other means, remove all fine-grained material that may be clinging to gravel sizes, taking care not to lose the fine-grained material.

d. Separate all the material into the finer fraction and the oversize fraction as will be defined in the fill compaction control procedure. This division will either be on the minus $3 / 4-$ in. sieve or the minus No. 4 sieve.

e. Place the two fractions of the total sample in separate containers, weigh and determine the percent by total weight of oversize fraction and percent by total weight of finer fraction.

f. Recombine the two fractions, mixing thoroughly and taking care not to lose any of the material.

g. Add a sufficient weight of water to bring the total material to a water content approximately within the specified fill placement range. In calculating the quantity of water to add, consider the air-dry water content of the material to be one percent.

h. Thoroughly mix the added water into the sample. Place the wetted sample in sealed containers and determine the wet weight of the entire sample.

i. Allow the wetted sample to cure for at least 24 hours.

i. After the moist sample has cured, separate a sufficient portion of it over the sieve which defines the oversized/finer fractions to obtain a sufficient quantity of the finer fraction to determine its water content. Work out of the sealed container(s) as efficiently as possible taking appropriate measures to avoid drying of the materials during the extraction of the sample of the finer fraction. Be extremely careful not to lose any of the material.

k. Determine the water content of the specimen of finer fraction $\mathrm{W}_{\mathrm{f}}$ obtained in $\mathrm{i}$. above by oven-drying as per EM 1110-2-1906. Retain the record of its wet $W_{w f}$ and dry $W_{d f}$ weights.

1. Determine the wet $W_{w r}$ and oven-dry $W_{d r}$ weights of the remainder of the total sample. If oven size or capacity will not accommodate the entire remainder of the total sample, it may be 
dried in portions. Take care not to lose any of the material and keep the portions awaiting drying in a sealed container.

m. Calculate the water content of the total sample $W_{t}$ as follows:

$$
W_{t}=\frac{\left(W_{w f}-W_{d f}\right)+\left(W_{w r}-W_{d r}\right)}{W_{d f}+W_{d r}} \times 100 \text { percent }
$$

n. Rearrange Equation B1 above to solve for the water content of the oversize fraction $W_{c}$ as follows:

$$
W_{c}=\frac{W_{t}-f W_{f}}{C}
$$

o. Substitute the following values into Equation (B1c):

(1) The percent finer fraction determined in step $\underline{\text { e. above }}$

(2) The percent oversized fraction determined in step e. above

(3) The water content of the finer fraction $W_{f}$ expressed as a percent determined in step $\underline{i}$. above

(4) The water content of the total sample $W_{t}$ expressed as a percent determined from step $\underline{\mathrm{k}}$. above

p. Solve Equation B1c for the water content of the oversized fraction $W_{c}$.

6. Note that the procedure above avoids the impractical task of separating the moist total sample into finer and oversized fractions such that no wet, fine-grained material adheres to the oversized fraction. It is this probability of adhering, wet, fine-grained material which negates a direct attempt to measure the water content of the oversized particles by simply oven-drying that fraction.

7. The above procedure applied to representative samples spanning the range in gradation of the earth-rock materials to be placed in the fill should yield a better general knowledge of the actual water content of the oversized material to be used with Equations B1 or Bla during the compaction control operations in the field. 